\title{
THERMO-ACOUSTIC CHARACTERIZATION OF THE BURNER-TURBINE INTERFACE IN A CAN-ANNULAR COMBUSTOR USING CFD
}

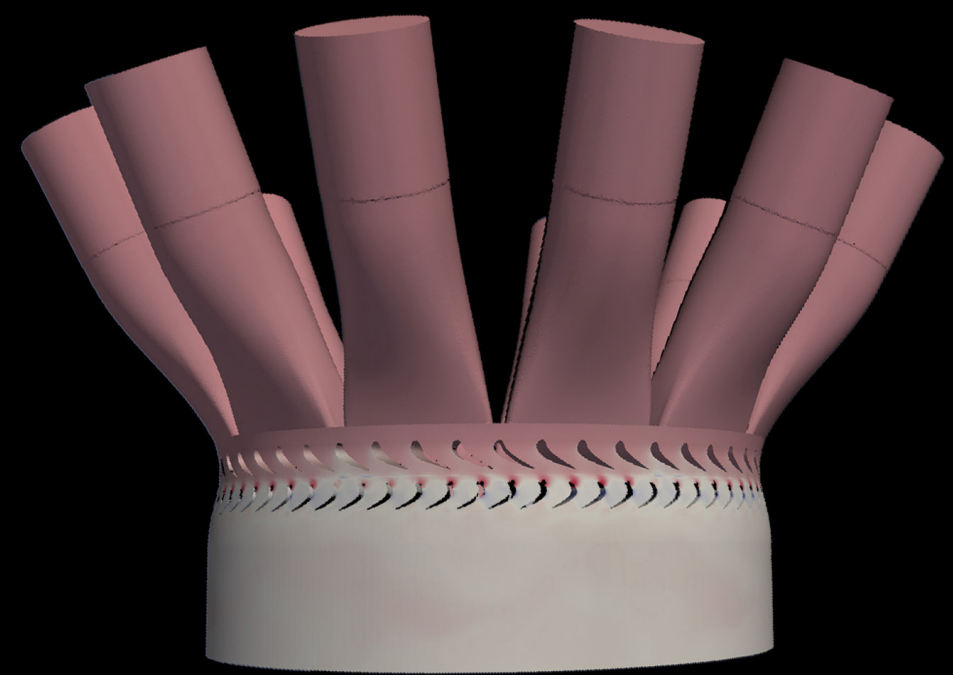

Federica Farisco 


\section{THERMO-ACOUSTIC CHARACTERIZATION OF THE BURNER-TURBINE INTERFACE IN A CAN-ANNULAR COMBUSTOR USING CFD}


The numerical research presented in this work has been funded by the European Community's Seventh Framework Programme (FP7, 2007-2013),

PEOPLE programme, under the grant agreement No FP7-290042(COPAGT project).

Thermo-acoustic characterization of the burner-turbine interface in a can-annular combustor using CFD

Farisco, Federica

PhD thesis, University of Twente, Enschede, The Netherlands, December 2016

Copyright (c) 2016 by Federica Farisco, Enschede, The Netherlands

No part of this publication may be reproduced by print, photocopy or any other means without the permission of the copyright owner.

ISBN: 978-90-365-4251-7

DOI: $10.3990 / 1.9789036542517$ 


\title{
THERMO-ACOUSTIC CHARACTERIZATION OF THE BURNER-TURBINE INTERFACE IN A CAN-ANNULAR COMBUSTOR USING CFD
}

\author{
DISSERTATION
}

to obtain

the degree of doctor at the University of Twente, on the authority of the rector magnificus,

Prof. dr. T.T.M. Palstra,

on account of the decision of the graduation committee, to be publicly defended

on Friday $2^{\text {nd }}$ of December 2016 at 11:00 h

by

Federica Farisco

born on March 19th, 1986

in Prato, Italy 
The dissertation is approved by:

Prof.dr.ir. T.H. Van der Meer Promotor

Dr.ir. J.B.W. Kok Co-promotor 


\section{Composition of the graduation committee:}

Chairman and secretary:

Prof.dr. G.P.M.R. Dewulf

University of Twente

Promotor:

Prof.dr.ir. T.H. Van der Meer

University of Twente

Co-promotor:

Dr.ir. J.B.W. Kok

University of Twente

Members:

Prof.dr.ir. A. de Boer

University of Twente

Prof.dr.ir. H.W.M. Hoeijmakers

University of Twente

Prof.dr.ir. I. Lopez Arteaga

Eindhoven University of Technology

Prof.dr.ir. B.J. Boersma

Delft University of Technology

Dr. L. Panek

Siemens 



\section{Acknowledgements}

As first, I would like to express my gratitude to my advisor Dr. Lukasz Panek for the continuous support of my PhD study and related research, for his patience, motivation and knowledge. His guidance helped me during the time of research and writing of papers and thesis.

Besides my advisor, I would like to thank my University professor Dr. Jim Kok for providing his insightful comments and encouragement, but also for the hard questions which led me to widen my research from various perspectives.

My sincere thanks goes also to Dr. Bertram Janus, who provided me the opportunity to be part of his team at Siemens AG in Berlin and who gave access to computational power in Jülich.

I would also like to thank my COPA-GT group who gave me constructive comments and warm encouragement.

Last but not least, I would like to thank my family: my parents Sara and Raimondo, my sister Leda and my aunt Teresa for continuous moral support and my life in general.

Together with my family, a precious and special thanks goes to my boyfriend Simon, constantly next to me since a few years, supporting me to front all difficult moments during my daily life and enjoing with me the nice experiences.

Without their guidance and persistent help this dissertation would not have been possible. 


\section{Publications}

- F. Farisco, S. Rochhausen, M. Korkmaz, M. Schroll "Validation of flow field and heat transfer in a two-pass internal cooling channel using different turbulence models" Proceedings of ASME Turbo Expo 2013, June 3-7, 2013, San Antonio, Texas, USA. GT201395461

- F. Farisco, L. Panek, B. Janus, J.B.W.Kok "Numerical investigation of the thermoacoustic influence of the turbine on the combustor" Proceedings of ASME Turbo Expo 2015, June 15-19, 2015, Montreal, Canada. GT2015-42071

- F. Farisco, L. Panek, J.B.W.Kok, J. Pent and R. Rajaram "Thermo-acoustic coupling in can-annular combustors - a numerical investigation" ICSV22 The 22nd International Congress on Sound and Vibration, July 12-16, 2015, Florence, Italy

- F. Farisco, L. Panek, J.B.W.Kok "Thermo-acoustic cross-talk between cans in a canannular combustor" International Symposium: Thermoacoustic Instabilities in Gas Turbines and Rocket Engines: Industry Meets Academia, May 30th-June 2nd, 2016, TUM Institute for Advanced Study - Munich, Germany

- F. Farisco, L. Panek, J.B.W.Kok "Numerical Simulation of Sound Propagation through the Can-Annular Combustor Exit" submitted to Journal of Sound and Vibration

- F. Farisco, L. Panek, J.B.W.Kok "Thermo-acoustic cross-talk between cans in a canannular combustor" submitted to International Journal of Spray and Combustion Dynamics 


\section{Abstract}

Thermo-acoustic instabilities in high power density gas turbine engines need to be predicted and understood in order to avoid unexpected shutdown events or engine failure. To predict these instabilities, the acoustic behavior of the combustion system needs to be analyzed.

The presented dissertation is focused on the combustor-turbine interaction for acoustic waves. The first part of the study is based on the acoustic reflection coefficient analysis, where the region of interest is located at the interface between the can-type combustion chamber and the first turbine stage. Simplified two-dimensional (2D) geometries and the corresponding three-dimensional (3D) engine alike turbine design have been investigated. The real engine case consists of a can-annular combustion chamber sector and the first vane section. Numerical simulation methods have been used in order to allow the analysis of complex geometries. Compressible Large Eddy Simulation (LES) resolving acoustic scales and providing accurate flow conditions is applied based on the open source Computational Fluid Dynamics package OPENFOAM. A forced response approach is applied imposing a sound wave excitation at the inlet of the combustion chamber. The applied Non-Reflecting Boundary Conditions (NRBC) are verified for correct behavior and plausibility of the acoustic model set up. Multi-harmonic excitation with small amplitudes is used to preserve linearity. The post-processing for the geometries is performed using the two-microphone and the multi-microphone method in order to calculate the reflection coefficient and the acoustic impedance taking into account the effects of the mean flow. The numerical results obtained are compared to analytical formulae in order to test the validity of both approaches for the chosen geometries.

In the second part of the work, the objective is to investigate the acoustic coupling between the cans, focusing on the turbine inlet section where the cans connect. After the previous analysis of $2 \mathrm{D}$ and $3 \mathrm{D}$ configurations consisting of transition part and first turbine vane row, the complexity has been increased focusing also on the interaction between two neighboring cans. While the annular combustor design is expected to suffer from the occurrence of transverse waves and burner-to-burner acoustic interaction, the can-annular combustor design should be less vulnerable to transverse waves and acoustic burner-to-burner interaction, as the burners are acoustically coupled only by means of the turbine stator stage and the plenum. Measurements in such machines, however, indicate that the pressure modes in neighboring cans synchronize and oscillate in or out of phase. This fact implies the existence of non-negligible cross-talk between neighboring cans and it justifies the usage of numerical methods in order to validate and confirm the experimental results obtained. A forced response approach is used also for this analysis. A can-can transfer function has been computed in this case in order to evaluate the percentage of the pressure waves transferred to the second can (can-can transfer function). A study of the reflection coefficient has been performed together with the investigation of the transfer function between the cans. Simplified 2D and 3D equivalent systems are investigated pointing out comparisons between the different configurations. The numerical outcomes will assist to find possible solutions able to reduce the cross-talk effect and they will underline the accurate agreement between correspondent 2D and 3D configurations, justifying the analysis of $2 \mathrm{D}$ systems to reduce CPU time.

Finally an extension of the previous studies from purely static geometries to moving components has been investigated. An extension of the current solver into a moving-mesh combustion solver has been developed together with the generation of a mesh. As an outlook of the study, a final simulation with the 3D generated mesh needs to be completed with the aim to 
post-process the acoustics of the case. The method used to generate this final 3D mesh could be then used to couple more different gas turbine components, leading to a more accurate analysis of the interaction between various engine parts. 


\section{Samenvatting}

In gas turbine motoren (die een hoge vermogensdichtheid hebben) kunnen thermo-akoestische instabiliteiten optreden, die kunnen leiden tot een noodstop van de motor en tot zeer grote schade indien de noodstop te laat wordt gemaakt. Teneinde deze instabiliteiten te kunnen voorspellen, dient het akoestisch gedrag van het verbrandingssysteem te worden geanalyseerd. In dit proefschrift ligt de focus op de propagatie van akoestische golven door het domein van de verbrandingskamer en de turbine, en de interactie van de akoestische velden in deze beide componenten. Het eerste deel van deze studie richt zich op de analyse van de akoestische reflectie coefficient van golven op de overgang van de busvormige verbrandingskamer naar de eerste trap van de turbine. Bezien vanuit werkelijke motor ontwerpen zijn eerst vereenvoudigde tweedimensionale geometrien en daarna meer met de motor overeenkomende tweeen driedimensionale turbine ontwerpen onderzocht. De laatste categorie bestond uit een busannulaire verbrandingskamer sectie en een sectie met daarin de eerste rij stator schoepen. Numerieke simulatie methoden met aangepaste rekenroosters zijn toegepast om ingewikkelde geometrien te kunnen onderzoeken. Voor de simulatie van de turbulente stroming, rekening houdend met de akoestische schalen en nauwkeurige stromingsvoorspelling, is het LES model toegepast in een compressibel rekenmodel als aanwezig in het Computational Fluid Dynamics pakket OpenFOAM. Een zogenaamde "fprced response" methode is toegepast waarbij een geluidsgolf excitatie is opgelegd op de inlaat van de verbrandingskamer. Op de open randen van het domein is een "Non Reflecting Boundary Condition" opgelegd. De cerrecte werking van deze conditie is onderzocht alsmede de geloofwaardigheid van de opzet van het akoestische model. De excitatie van de geluidsgolven is gedaan simultaan op meerdere frequenties en met lage amplitude. Dit laatste om verzekerd te zijn van lineair gedrag van het systeem. De verkregen resultaten van de simulatie van de systeem respons als functie van de tijd zijn geanalyseerd met behulp van de twee- en multimicrofoon methode om de reflectie coefficient en de akoestische impedantie te berekenen inclusief het effect van de stroming. De resultaten zijn vergeleken met beschikbare analytische uitdrukkingen teneinde te validiteit van beide analysemethoden te beoordelen. In de tweede heft van deze studie is de akoestische koppeling tussen twee verbrandingskamers onderzocht, met de aandacht op dat deel van de turbine sectie, waar beide verbrandingskamers met elkaar verbonden zijn. Na de analyse in het eerste deel van het proefschrift van $2 \mathrm{D}$ en $3 \mathrm{D}$ configuraties bestaande uit het "transition piece" en de eerste rij stator schoepen van de turbine, is nu de complexiteit verhoogd door de mogelijke interactie van geluidsgolven tussen twee verbrandingskamers. In het conventionele annulaire verbrandingskamer ontwerp kunnen transversale akoestische golven optreden met als gevolg een sterke interactie tussen meerdere branders. Voor het can annulaire ontwerp, als bestudeerd in deze thesis, was de verwachting dat de interactie tussen meerdere branders vrijwel afwezig zou zijn, omdat zij alleen maar een akoestische koppeling kunnen hebben via de turbine stator sectie en het brander stroomopwaartse plenum. Helaas hebben metingen in deze can annulaire machines laten zien dat de drukgolven in aangrenzende verbrandingskamers kunnen synchroniseren and in of uit fase kunnen oscilleren. Dit duidt er op dat er significante overspraak kan zijn tussen aangrenzende verbrandingskamers en numeriek onderzoek is gewenst om de waargenomen fenomenen te bevestigen en verklaren. Ook in deze studie is een forced response methode gebruikt. Een can-can overdrachtsfunctie kan worden berekend in dit geval om de fractie van de akoestische intensiteit van de eerste can naar de tweede can te voorspellen. Samen met deze overdrachtsfunctie is ook de reflectieco- 
efficient voor twee can verbrandingskamers onderzocht. Twee en driedimensionale systemen zijn onderzocht voor wat betreft de overdrachtsfunctie en reflectiecoefficient voor systemen met twee verbrandingskamers. De resultaten van deze numerieke analyse laten zien dat er goede overeenkomsten zijn tusse de twee en driedimensionale configuraties, wat rechtvaardigt in het vervolg twee dimensionale modellen te gebruiken, wat veel rekentijd bespaart. Hopelijk zullen deze nieuwe inzichten leiden tot oplossingen om de overspraak tussen twee verbrandingskamers te verminderen. Het proefschrift sluit af met een aanzet naar het vervolg van deze studie, namelijk het in rekening brengen van de effecten van de turbine rotor onmiddellijk stroomafwaarts van de stator. Om dit te kunnen doen is een eerste opzet gemaakt voor uitbreiding van de huidige rekenmethode zodanig dat deze een combinatie kan hanteren van een roterend/statisch rekenrooster voor een compressibele stroming. Indien dit in een vervolg van deze studie volledig functioneel gemaakt wordt, dan kan samen met de in de huidige studie ontwikkelde akoestische analyse methoden, een nauwkeurige voorspelling gedaan worden van de akoestische interactie tussen verschillende gas turbine motor componenten. 


\section{Contents}

$\begin{array}{ll}\text { List of Figures } & \text { ix }\end{array}$

List of Tables $\quad$ xii

$\begin{array}{ll}\text { 1. Introduction } & \mathbf{1}\end{array}$

1.1. Introduction to the problem . . . . . . . . . . . . . . . 1

1.1.1. Motivation and aim of the study . . . . . . . . . . . . . 2

1.2. Combustor-turbine acoustic interaction - literature overview . . . . . . . . . 4

1.3. Thesis outline . . . . . . . . . . . . . . . . 7

2. Theoretical background - numerical method applied 9

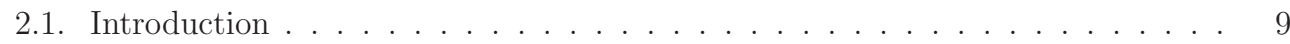

2.2. Propagation of waves in ducts . . . . . . . . . . . . . . . . . . . . . . . . . . . . . . . . . . . . . . .

2.2.1. The conservation laws . . . . . . . . . . . . . . . 10

2.2.2. Linearization of Euler equations . . . . . . . . . . . . . . . . 11

2.2.3. Acoustic wave equation . . . . . . . . . . . . . . . . . . . . . . . . . . . . . 11

2.2.4. Wave propagation in circular ducts . . . . . . . . . . . . . . . 12

2.2.5. Cut-off frequency . . . . . . . . . . . . . . . . . . . . . . . . . . . . . . . . . . . . . .

2.2.6. Plane waves in a duct . . . . . . . . . . . . . . . . . . . . . . . . . . . . . . . . . .

2.2.7. Acoustic impedance . . . . . . . . . . . . . . . . . . . . . 14

2.3. Thermo-acoustic instabilities analysis . . . . . . . . . . . . . . . . . 14

2.3.1. Control methods for thermo-acoustic instabilities . . . . . . . . . . . 15

2.4. LES and CFD code applied . . . . . . . . . . . . . . . . . 16

2.5. Boundary conditions . . . . . . . . . . . . . . . . . . 17

2.5.1. Evaluation of Non-Reflecting Boundary Conditions . . . . . . . . . . 19

2.5.2. Cyclic boundary conditions . . . . . . . . . . . . . . 22

2.6. Post-processing - methods overview . . . . . . . . . . . . . . . . . 23

2.6.1. Two-microphone method . . . . . . . . . . . . . . . . . 23

2.6.2. Limits of the method . . . . . . . . . . . . . . . . . . . . 25

2.6.3. Amplitude evaluation method . . . . . . . . . . . . . 25

2.6.4. Multi-microphone method . . . . . . . . . . . . . . 27

3. Thermoacoustic Influence of the Turbine on the Combustor - validation of tool chain 29

3.1. Introduction . . . . . . . . . . . . . . . . . . . . . 29

3.2. Investigated configurations . . . . . . . . . . . . . . . . 30 
3.3. Numerical method . . . . . . . . . . . . . . . . . . . . 30

3.3.1. Solver and boundary conditions . . . . . . . . . . . . . . . . 30

3.3.2. Meshing . . . . . . . . . . . . . . . . . . . . . . . . . . . . . . . . . . . . . . .

3.4. Two-microphone method . . . . . . . . . . . . . . . 33

3.4.1. Two-microphone method validation . . . . . . . . . . . . . . 33

3.5. Analytical solutions . . . . . . . . . . . . . . . . . . . . . . . . . . . . . . . 35

3.5.1. Low frequency approximation . . . . . . . . . . . . . . 35

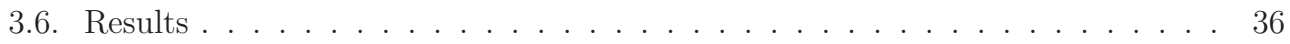

3.6.1. Convergent nozzle . . . . . . . . . . . . . . . . . . 36

3.6.2. Divergent nozzle . . . . . . . . . . . . . . . . . . . . . . . . 39

3.6.3. Convergent-divergent nozzle . . . . . . . . . . . . . . . . . . 39

3.6.4. 2D stator vane . . . . . . . . . . . . . . . . . . . . . . . . 44

3.6.5. Transfer matrix method extension to flow conditions . . . . . . . . . 44

3.7. Conclusion . . . . . . . . . . . . . . . . . . . . . . 47

\section{Numerical Simulation of Sound Propagation through the Can-Annular Combustor}

Exit $\quad 51$

4.1. Introduction . . . . . . . . . . . . . . . . . . . 51

4.2. Investigated Configurations . . . . . . . . . . . . . . . . . . . 52

4.3. Numerical Method . . . . . . . . . . . . . . . . . . . . . . . . . . . . . . . . . . . . . . . 52

4.3.1. LES Compressible Solver . . . . . . . . . . . . . . . . . . . . . . . . . . . . . . . . . . 52

4.3.2. NRBC and Periodicity . . . . . . . . . . . . . . . . . . 52

4.3.3. Flow . . . . . . . . . . . . . . . . . . . . 52

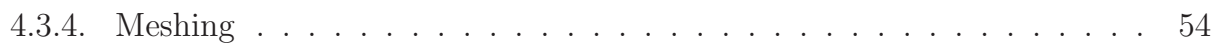

4.3.5. Forced Response Approach . . . . . . . . . . . . . . . . . . 54

4.3.6. Methods applied in the post-processing . . . . . . . . . . . . . 54

4.3.7. Validity of planar wave assumption . . . . . . . . . . . . . . . 54

4.4. Analytical solutions . . . . . . . . . . . . . . . . . . 56

4.4.1. Formulation of Cumpsty and Marble . . . . . . . . . . . . . 56

4.5. Results . . . . . . . . . . . . . . . . . . . 57

4.5.1. Flow Field . . . . . . . . . . . . . . . . . . 58

4.5.2. Reflection Coefficient . . . . . . . . . . . . . . . . 58

4.5.3. Analysis of the sound propagation direction . . . . . . . . . . . . 63

4.6. Conclusion . . . . . . . . . . . . . . . . . . . . . . . . . . . . . .

5. Thermo-acoustic Coupling in Can-Annular Combustors 71

5.1. Introduction . . . . . . . . . . . . . . . . . 71

5.2. Investigated configurations . . . . . . . . . . . . . . . . . . . . . . . . . . . . . .

5.3. Numerical method . . . . . . . . . . . . . . . . . . . . . . . 73

5.3.1. LES Compressible Solver . . . . . . . . . . . . . . . . . . . . 73

5.3.2. NRBC and Periodicity . . . . . . . . . . . . . . . . 73

5.3.3. Flow . . . . . . . . . . . . . . . . . . . 76

5.3.4. Meshing . . . . . . . . . . . . . . . . 76

5.3.5. Forced response approach . . . . . . . . . . . . . . 76

5.3.6. Post-processing - Methods applied . . . . . . . . . . . . . . . . 77 
5.4. Results . . . . . . . . . . . . . . . . . . . . . . . 77

5.4.1. NRBC analysis . . . . . . . . . . . . . . . . . 77

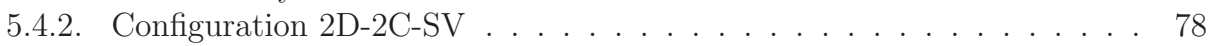

5.4.3. Configuration 2D-2C-DV . . . . . . . . . . . . . . . 80

5.4.4. Configuration 3D-2C-DV with single side excitation . . . . . . . . 84

5.4.5. Configuration 3D-DV with symmetric excitation . . . . . . . . . 84

5.4.6. Resonance study . . . . . . . . . . . . . . . . . . . . 87

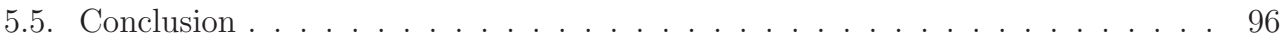

$\begin{array}{ll}\text { 6. Conclusion and Outlook } & 101\end{array}$

6.1. General introduction . . . . . . . . . . . . . . . . . . . . . 101

6.2. Thermoacoustic Influence of the Turbine on the Combustor - validation of tool chain . . . . . . . . . . . . . . . . . . . . . . . 101

6.3. Numerical Simulation of Sound Propagation through the Can-Annular Combustor Exit . . . . . . . . . . . . . . . . . . . . . . 102

6.4. Thermo-acoustic cross-talk between cans in a can-annular combustor . . . . 102 6.4.1. Outlook . . . . . . . . . . . . . . . . . . . . . . . . 103

6.5. Characterization of rotor effects on can-annular combustor acoustics . . . . . 103

6.5.1. Introduction . . . . . . . . . . . . . . . . 103

6.5.2. Motivation for needing a rotating mesh capability - problem description 103

A. Appendix

A.1. Example of numerical setting applied . . . . . . . . . . . . . . . 105

A.2. Procedure how to generate cyclic patches . . . . . . . . . . . . . . . 109

A.3. Procedure how to build a 2D mesh with SnappyHexMesh . . . . . . . . . 110

A.4. Study performed concerning rotating meshes . . . . . . . . . . . . . . 112

A.4.1. Periodic sector cylinder - Stator-Rotor-Stator application . . . . . . . 112

A.4.2. Cylinder rotating - OpenFOAM 1.6ext . . . . . . . . . . 112

A.4.3. Turbo Passage rotating - partial overlap GGI - OPEnFOAM 1.6ext . . 114

A.4.4. Error compilation . . . . . . . . . . . . . . . . . . . 115

A.4.5. Complex configurations . . . . . . . . . . . . . . . 116

A.5. 3D moving mesh Stator-Rotor generation . . . . . . . . . . . . . 116

A.6. Characterization of rotor effects on can-annular combustor acoustics . . . . . 122

A.6.1. Introduction . . . . . . . . . . . . . . . . . . . . 122

A.6.2. Motivation for an extension of the current solver . . . . . . . . 122

A.6.3. Developed moving-mesh combustion solver and 3D mesh generation . . 124

A.6.4. Outlook . . . . . . . . . . . . . . . . . . . . . . . . 124 


\section{List of Figures}

1.1. Damages caused by combustion instabilities in a burner configuration, see

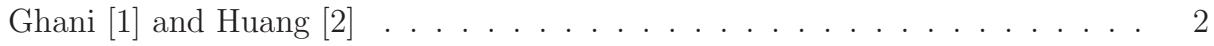

1.2. COPA-GT project structure . . . . . . . . . . . . . . . 3

1.3. Siemens configuration overview, see http://merkl-gmbh.de/revision-siemens-

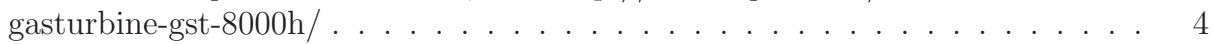

1.4. Can-can eigen-mode in a generic multi-can setup . . . . . . . . . . . . . 6

2.1. Acoustic pressure in a cylindrical duct for four modal components (m:n), see Lahiri $[3] \ldots \ldots \ldots \ldots$

2.2. Different modes depending on the geometry, see Ghani [1] . . . . . . . . . . 16

2.3. Impact of the boundary condition time scale on the reflection coefficient, see Beck $[4] \ldots \ldots \ldots \ldots \ldots$

2.4. Pipe geometry overview . . . . . . . . . . . . . . . . . . . 19

2.5. Distribution of velocity and pressure in case of RBC . . . . . . . . . . . . . . 20

2.6. Distribution of velocity and pressure in case of NRBC . . . . . . . . . . . . 21

2.7. Example of cyclic patches, see Jasak [5] . . . . . . . . . . . . . . . . . . . 22

2.8. Example of post-processing techniques applied, see Hanraths [6] . . . . . . . . 24

2.9. Two Microphone Method with mean flow, see Munjal \& Doige [7] . . . . . . . 24

2.10. Wave length over frequency for typical temperatures, can diameter for comparison 25

2.11. Stack of VTK planes from OpenFOAM . . . . . . . . . . . . . . . 27

3.1. Nozzle geometries analyzed in 2D. The red marks depict the microphones used for the two-microphone method . . . . . . . . . . . . . . . . . 31

3.2. Two-microphone method validation performed choosing two different microphone distances $s$ below and above the criterion $s<\lambda / 2 \ldots \ldots$. . . . . 34

3.3. Reflection coefficient for convergent nozzle: no flow conditions . . . . . . . . . 37

3.4. Reflection coefficient for convergent nozzle: flow conditions . . . . . . . . . . . 38

3.5. Reflection coefficient for divergent nozzle: no flow conditions . . . . . . . . . . 40

3.6. Reflection coefficient for divergent nozzle: flow conditions . . . . . . . . . . . . 41

3.7. Reflection coefficient for convergent-divergent nozzle: no flow conditions . . . . 42

3.8. Reflection coefficient for convergent-divergent nozzle: flow conditions . . . . . 43

3.9. Reflection coefficient for $2 \mathrm{D}$ case with vane: no flow conditions . . . . . . . . . 45

3.10. Reflection coefficient for $2 \mathrm{D}$ case with vane: flow conditions . . . . . . . . . . . 46

3.11. Network model used for convergent-divergent nozzle CDN . . . . . . . . . . 47

3.12. Reflection coefficient comparison between analytical formulation and numerical results obtained for CDN-CN-DN with flow . . . . . . . . . . . . . . 48 
4.1. Overview of investigated geometries with definition of the boundary conditions

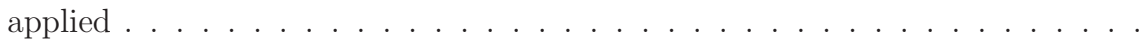

4.2. Reflection coefficient magnitude for 3D configuration - multi-microphone method compared to two-microphone method . . . . . . . . . . . . . . . 55

4.3. Reflection coefficient phase for 3D configuration - multi-microphone method compared to two-microphone method . . . . . . . . . . . . . . 55

4.4. Analytical formula in Eq.(4.1) applied to $2 \mathrm{D}$ single vane . . . . . . . . . . . . 57

4.5. Distribution of the Mach number in the stator passage . . . . . . . . . . . . . 59

4.6. Distribution of the wave impedance in the stator passage . . . . . . . . . . 60

4.7. 2D versus 3D configurations - Reflection coefficient spectrum at outlet . . . . . 61

4.8. $2 \mathrm{D}$ versus $3 \mathrm{D}$ configurations - Reflection coefficient phase spectrum at outlet . 61

4.9. Reflection coefficient magnitude for $2 \mathrm{D}$ and $3 \mathrm{D}$ cases compared to analytical formulation in Eq.(3.3): no flow . . . . . . . . . . . . . . . . 62

4.10. Reflection coefficient phase for $2 \mathrm{D}$ and $3 \mathrm{D}$ cases compared to analytical formulation in Eq.(3.3): no flow . . . . . . . . . . . . . . . . . . . 63

4.11. Reflection coefficient magnitude for $2 \mathrm{D}$ and $3 \mathrm{D}$ cases compared to analytical formulation in Leyko et al.[8] in Eq.(4.1): flow . . . . . . . . . . . . . . . . . . 64

4.12. Reflection coefficient phase for $2 \mathrm{D}$ and $3 \mathrm{D}$ cases compared to analytical formulation in Leyko et al.[8] in Eq.(4.1): flow . . . . . . . . . . . . . . . . 64

4.13. $2 \mathrm{D}$ single vane flow study - Reflection coefficient magnitude . . . . . . . . . 65

4.14. 2D single vane flow study - Reflection coefficient phase . . . . . . . . . . . . 65

4.15. 2D versus 3D configurations - Reflection coefficient magnitude . . . . . . . . . 66

4.16. $2 \mathrm{D}$ versus $3 \mathrm{D}$ configurations - Reflection coefficient phase . . . . . . . . . 66

4.17. $2 \mathrm{D}$ vane sound propagation angle study . . . . . . . . . . . . . . . 67

4.18. $2 \mathrm{D}$ vane sound propagation angle analysis . . . . . . . . . . . . 68

5.1. 2D configurations with SV and different vane locations . . . . . . . . . . 74

5.2. 2D-2C-SV-RG overview with probe positions and waves directions . . . . . . 74

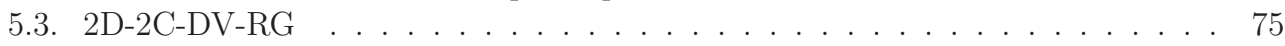

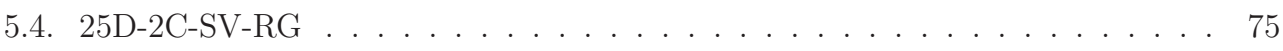

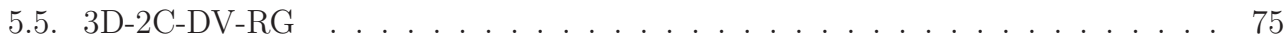

5.6. Boundary conditions applied for the cases $2 \mathrm{D}-2 \mathrm{C}-\mathrm{SV}-\mathrm{RG}$ as example $\ldots$. . . 76

5.7. Reflection coefficient spectrum at outlet . . . . . . . . . . . 78

5.8. Transmission coefficient $X$ evaluation for 2D-2C-SV configurations with different gaps and Mach numbers . . . . . . . . . . . . . . . . . . . . . 79

5.9. Acoustic field reconstruction for $2 \mathrm{D}-2 \mathrm{C}-\mathrm{SV}-\mathrm{RG}$ with low frequencies and $M \approx 0.481$

5.10. Acoustic field reconstruction for 2D-2C-SV-RG with high frequencies and $M \approx$

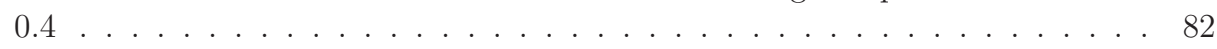

5.11. Cut-off frequency plotted over modes . . . . . . . . . . . . . . . 83

5.12. Transmission coefficient $X$ for $25 \mathrm{D}-2 \mathrm{C}-\mathrm{SV}-\mathrm{RG}$ compared with the corresponding 2D-2C-SV-RG with $M \approx 0.4 \ldots \ldots \ldots . \ldots . \ldots$

5.13. Acoustic field reconstruction for $2 \mathrm{D}-2 \mathrm{C}-\mathrm{DV}-\mathrm{RG}$ with low frequencies and $M \approx$

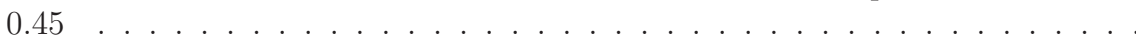

5.14. Acoustic field reconstruction for $2 \mathrm{D}-2 \mathrm{C}-\mathrm{DV}-\mathrm{RG}$ with high frequencies and $M \approx$

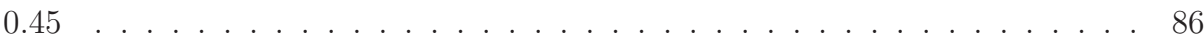

5.15. Transmission coefficient $X$ for 2D-2C-DV with different gaps and $M=0.45$. 87 
5.16. Transmission coefficient at the inlet estimated for $3 \mathrm{D}$ and $2 \mathrm{D}$ configurations with single side excitation and $M=0.7 \ldots \ldots \ldots$. . . . . . . 88

5.17. Acoustic field reconstruction at low frequencies for 3D-2C-DV-RG configura-

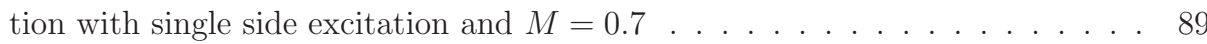

5.18. Acoustic field reconstruction at high frequencies for 3D-2C-DV-RG configuration with single side excitation and $M=0.7 \ldots \ldots \ldots$. . . . . . . 90

5.19. Overview of comparable configurations . . . . . . . . . . . . . . . 91

5.20. Reflection coefficient at the inlet estimated for 3D-1C-DV-RG and 3D-2C-DVRG configurations with symmetric excitation with $M \approx 0.7 \ldots \ldots . . . . .992$

5.21. Reflection coefficient at the inlet estimated for 3D-1C-DV-RG and 3D-2C-DVRG configurations with symmetric excitation with $M=0 \ldots \ldots 3$

5.22. Acoustic field reconstruction at low frequencies for 3D-DV-RG configuration with symmetric excitation with $M=0.7 \ldots \ldots . \ldots . \ldots . \ldots 94$

5.23. Acoustic field reconstruction at high frequencies for 3D-2C-DV-RG configura-

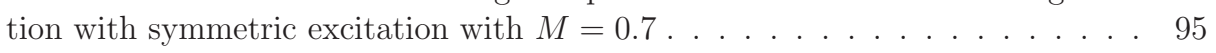

5.24. Transmission coefficient comparisons between 2D-2C-SV-RG and 2D-2C-SVRG with shorter vanes . . . . . . . . . . . . . . . . . . . 97

5.25. Pressure evaluation for case 2D-2C-SV-RG with single side excitation at $f=$ $630 \mathrm{~Hz} \ldots \ldots \ldots \ldots . \ldots \ldots$

5.26. Wave amplitudes evaluation . . . . . . . . . . . . . . . . . . . . 99

A.1. FlattenMesh of linearDirection extrusion of nonflat patch . . . . . . . . . . 112

A.2. LinearDirection extrusion of nonflat patch . . . . . . . . . . . . . . 113

A.3. Inlet swirl detail . . . . . . . . . . . . . . . . . . . . . . . . . . . . . . . . . . . . . . . . . . . . . . .

A.4. Turbo passage rotating overview . . . . . . . . . . . . . . . . . . . . . . . . . . . . . . . . . . . .

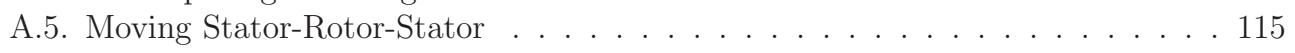

A.6. Cylinder Stator-Rotor-Stator . . . . . . . . . . . . . . . 116

A.7. Cyclic AMI interfaces overview in the final mesh obtained . . . . . . . . . . . 122

A.8. An example of cyclic AMI interfaces . . . . . . . . . . . . . . . . . . . . . . . . . . . . . . . . . . . . .

A.9. 3D mesh overview . . . . . . . . . . . . . . . . . . . . . . . . . . . . . . . . . . . . . . . . . . . . . . . . . . . . . .

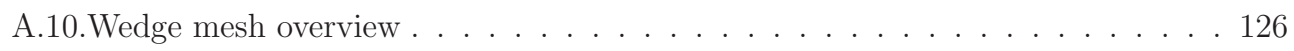

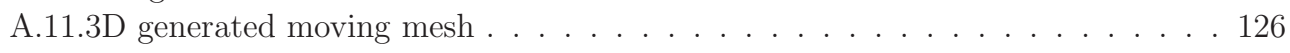

A.12.3D generated final moving mesh . . . . . . . . . . . . . . 127 


\section{List of Tables}

3.1. Convergent-divergent nozzle: Flow conditions . . . . . . . . . . . . . 32

3.2. Convergent nozzle: Flow conditions . . . . . . . . . . . . . . . . . . 32

3.3. Divergent nozzle: Flow conditions . . . . . . . . . . . . . . . . . . 33

3.4. 2D stator vane: Flow conditions . . . . . . . . . . . . . . . . . . 33

5.1. Investigated configurations - Different vane locations . . . . . . . . . . . 73

5.2. Investigated configurations - Mach conditions . . . . . . . . . . . 73 
List of Tables 


\section{Introduction}

\subsection{Introduction to the problem}

Lean premixed combustion technology became state-of-the art in gas turbine engines for power generation. These systems allow higher efficiency, reduction of carbon monoxide (CO) and nitrogen oxides (NOx). NOx emissions increase fast once the flame temperature achieves values higher than $1500{ }^{\circ} \mathrm{C}$. Injection of water or steam was introduced in the combustion chamber to reduce the flame temperature but this led to higher engine complexity connected to increased costs. The annular and can-annular designs substituted silo combustors to guarantee a lean combustion with reduced cooling air. The introduction of premixed air and fuel before entering the combustion chamber decreased the presence of hot spots. The flame front in premixed combustion can amplify acoustic oscillations to high amplitudes in limit cycle modes (see Roman Casado [9], Kubis [10] and Keller [11]). Due to unstable combustion, the interaction between heat release and acoustic field generate high amplitude pressure oscillations within the combustion chamber. The acoustic waves resulting from this process propagate downstream the combustion chamber and are then reflected by the boundaries back to the flame area. The phase difference between pressure and heat release oscillations can result in damped acoustic waves or extreme amplitudes that can cause damage to the engine (see Poinsot \& Veynante [12], Williams [13], Huang \& Yang [2]) as observed in Fig. 1.1. The 


\section{Introduction}
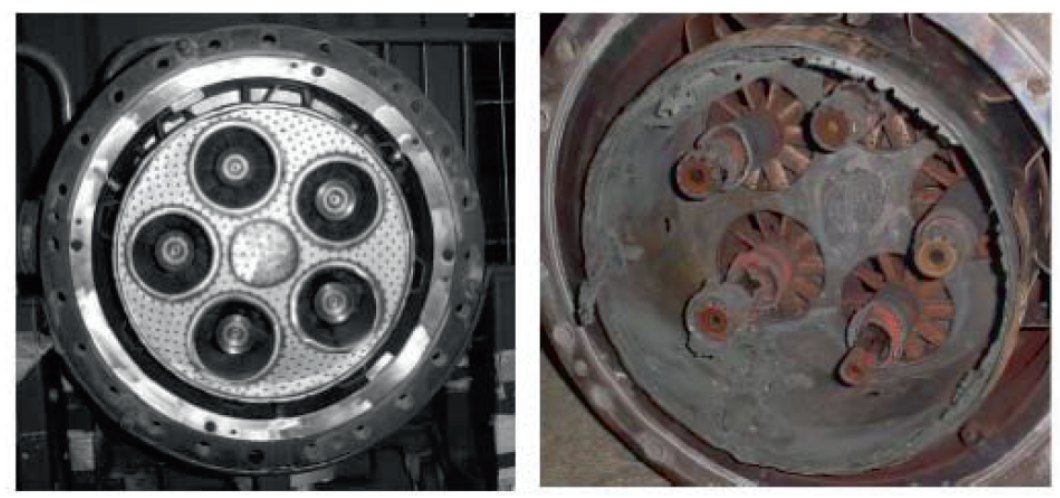

Figure 1.1.: Damages caused by combustion instabilities in a burner configuration, see Ghani [1] and Huang [2]

Rayleigh criterion (Rayleigh [14], Lieuwen [15], Nicoud \& Poinsot [16]) shows how a positive phase amplifies acoustic fluctuations that can on the other hand also be damped if pressure and heat release oscillations are out of phase. Knowledge of the acoustic behavior is required in order to understand and predict these instabilities. Mode shape and frequency of the pressure oscillations excited within the combustion chamber, need to be investigated.

This topic has been investigated as a part of the Marie Curie Project COPA-GT funded by the European Commission. The main aim of this project was to perform coupled simulations of the different gas turbine components taking into account all physics and component interaction. The research contributions of this work to the project are related to the Work Package (WP3) "Coupled Combustor/Turbine calculation", and the activities are in collaboration with the University of Twente and the Von Karman Institute for Fluid Dynamics (VKI).

The current Work Package is focused on the development of innovative and advanced numerical methods to investigate the combustor and gas turbine aero-thermal behavior. A general overview of the different topics developed by COPA-GT is shown in Fig. 1.2. Fig. 1.2 shows also the contributions to the various themes given by the industrial and academic partners involved in the project.

\subsubsection{Motivation and aim of the study}

The focus of this dissertation is on the thermo-acoustic behavior of the can-annular gas turbine combustor system currently applied at Siemens.

Although the can-annular combustor represents a simpler acoustic system compared to annular combustors, many aspects, especially the combustor/turbine interaction, are not investigated in-depth. In this Work Package the mechanically complex section between the can-end and the first turbine stage (stator) is investigated by numerical high fidelity time resolved three-dimensional (3D) methods, Large-Eddy-Simulations (LES). The idea is to start the analysis from simplified $2 \mathrm{D}$ geometries, comparing the numerical results obtained with 


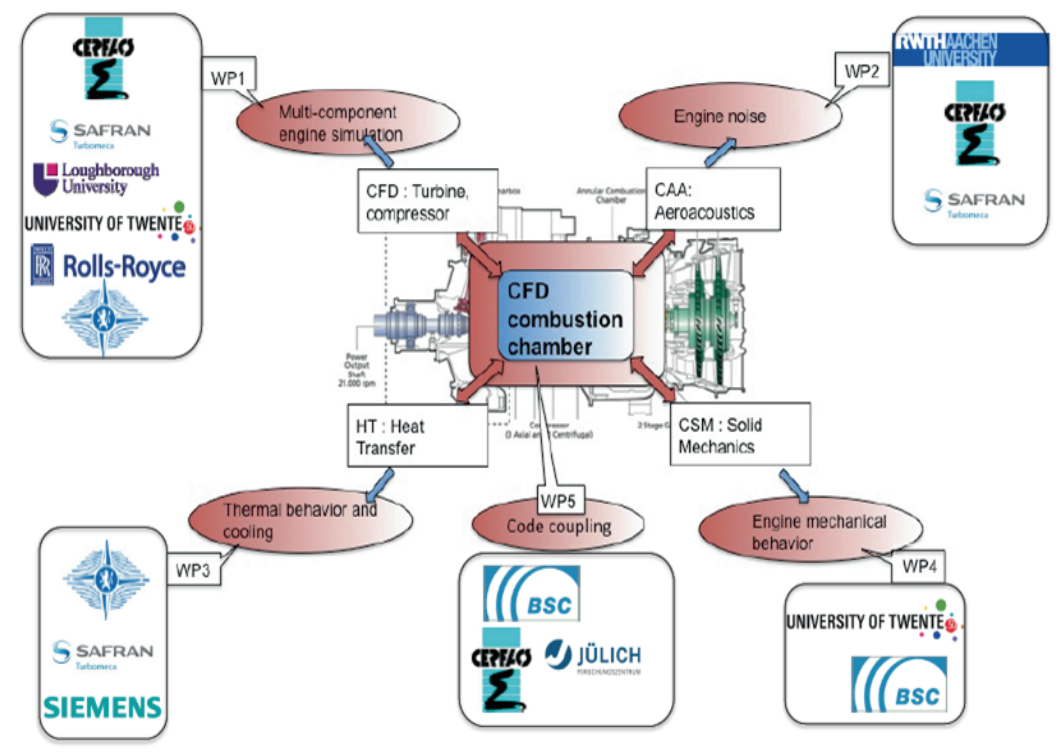

Figure 1.2.: COPA-GT project structure

analytical formulations.

The study proceeds increasing the complexity of the geometries analyzed arriving to investigate 3D configurations consisting of the transition piece at the outlet of the combustion chamber and the first turbine vane row. The following step is to study the acoustic interaction between two neighboring cans adding in the end the rotating components to the last 3D geometry. Compressible Large Eddy Simulation resolving acoustics is applied for all simulations based on the open source CFD code OpenFOAM. Calculations with acoustic excitation and Non-Reflecting Boundary Conditions (NRBC) at the computational inlet and outlet domains are carried out to verify the plausibility of the acoustic set up. A forced response approach is applied provoking a wave excitation at the inlet of the combustion chamber. Multi-harmonic excitation with small amplitudes is used to stay in the linear range.

Finally guidelines for the designer are formulated, proving that the cross-talk between the cans cannot be neglected and it is shown how to reduce it. The rotating components are included in the last part of this work. This last section represents the basis that will lead to perform a numerical simulation of different gas turbine components coupled. This will allow a complete evaluation of the interaction of various engine parts. The detailed achievements are pointed out in the following chapters. 


\subsection{Combustor-turbine acoustic interaction - literature}

\section{overview}

The correct modeling of acoustic oscillations in the latest lean premixed combustion technology requires a detailed knowledge of the boundary regions directly influencing the resonant phenomena. So it is necessary to understand the reflection behavior of the upstream and downstream side of the heat release zone.

The analysis of the reflection coefficient of the combustor and turbine interface is the focus area of this study.

The aim of the work concerns a numerical investigation of the acoustic behavior of geometries with a different level of complexity. The engine configuration to which this study is related is presented in Fig. 1.3. It is a can-annular stationary gas turbine. Clearly the multiple combustor cans can be seen. The exit passages of all cans together feed the turbine inlet over the circumference.

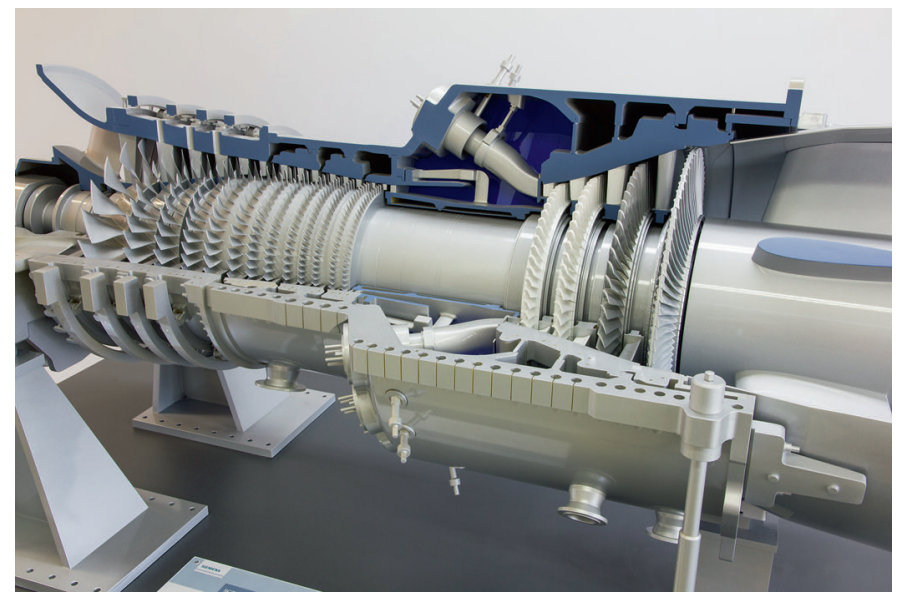

Figure 1.3.: Siemens configuration overview, see http://merkl-gmbh.de/revision-siemensgasturbine-gst-8000h/

In the numerical studies presented, the sound waves originating from thermo-acoustic instabilities in the real system are replaced by artificial ones using the forced response approach. An excitation has been applied at the inlet, and the resulting acoustic perturbations upstream and downstream of the turbine stator stage were analyzed. The current investigation focuses on the acoustic perturbations excluding entropy and vortical solution modes. It analyzes the acoustic reflection coefficient for a simplified $2 \mathrm{D}$ geometry in comparison to the results obtained for a complex geometry 3D model. The numerical data is compared to analytical solutions for quiescent and subsonic flow conditions. The innovation of this first study consists in obtaining data from numerical simulations (LES) of a realistic 3D case including the downstream section of a combustion can and the first vane row.

The reflection and transmission of sound, vortices and entropy perturbation through turbine 
blades or vanes has been studied in depth the past four decades. The investigations were focused on the explanation of the so called excess noise (Cumpsty \& Marble [17]) with the entropy fluctuations in the foreground, but also resulted in valuable formulations for the remaining solution modes. Marble [18] and Marble \& Candel [19] following the works of Tsien [20] and Crocco [21], studied and treated in one dimension the interaction of entropy and pressure waves in choked and unchoked nozzles. Cumpsty \& Marble [17] treated a system related closer to a gas turbine where perturbations are interacting with a turbine blade row. They have shown how direct and indirect combustion noise sources need to be taken into account and present predictions based on analytical models and empirical data. Kaji \& Okazaki [22], [23] have analyzed a method focused on the acceleration potential to study the sound transmission through a 2D rectilinear cascade. Review papers by Lamarque \& Poinsot [24] and Duran et al. [25] provide an overview of the experimental and numerical studies related to the reflection coefficient analysis. They represent inlets and outlets of chambers as one-dimensional ducts and evaluate impedances of subsonic and choked compact nozzles through analytical formulae and numerical methods using the Linearized Euler Equations (LEE). Furthermore the work of Leyko et al. [8] demonstrates the use of analytical models for $1 \mathrm{D}$ and $2 \mathrm{D}$ flows and the usage of the compact noise assumption for 1D models. Many models with finite chord-length of blades and finite spacing between blades in 1D and 2D have been analyzed but few real 3D cases have been studied until now. Work by Posson \& Roger [26] and Posson et al. [27] show a 3D cascade for entropy noise generation that takes into account cascade effects and finite chord neglecting the angle of deviation. Posson \& Roger [26] focus on the development of an analytical method for the sound transmission through an annular geometry of a blade row.

The second part of this dissertation concerns the analysis of the can-annular combustors interaction evaluating the can-can modes generated in these systems. After the previous analysis about 2D simplified configurations and 3D geometries consisting of transition part and first turbine vane row, the analyzed working domain has been increased focusing also on the interaction between two neighboring burners. Compared to the engines with annular combustors, the can-annular combustor design is expected to be less vulnerable to transversal waves. In this last case the burners are acoustically coupled only through the turbine stator stage and the plenum. The expectation was that this limited coupling of the flow domain would provide uncoupling of the acoustic behavior in two neighboring cans in comparison to an annular design. Measurements in these machines indicate surprisingly that the pressure modes in neighboring burners can synchronize and oscillate in phase or with opposite phase. This enables so called can-can modes that do not exsist in a single isolated can (see Fig. 1.4). This fact implies the existence of non-negligible can cross-talk as shown by Farisco et al. [28]. These observations indicate the limits of using a single combustor can in a test rig, to test the acoustics and coupling to combustion dynamics. The single can has different acoustic boundary conditions as compared to a system of coupled cans. Methods need to be proposed to verify stability analysis in modern combustors at the design level.

The analysis of the reflection coefficient of the combustor and turbine interface and the transmission coefficient evaluated between two neighboring cans are the focus area of this part of the thesis.

The aim of the study is to investigate which procedure could reduce the cross-talk, and to compare the numerical results obtained for $2 \mathrm{D}$ and $3 \mathrm{D}$ corresponding configurations. In the current study the reflection coefficient study is performed in parallel to a transmission coeffi- 


\section{Introduction}

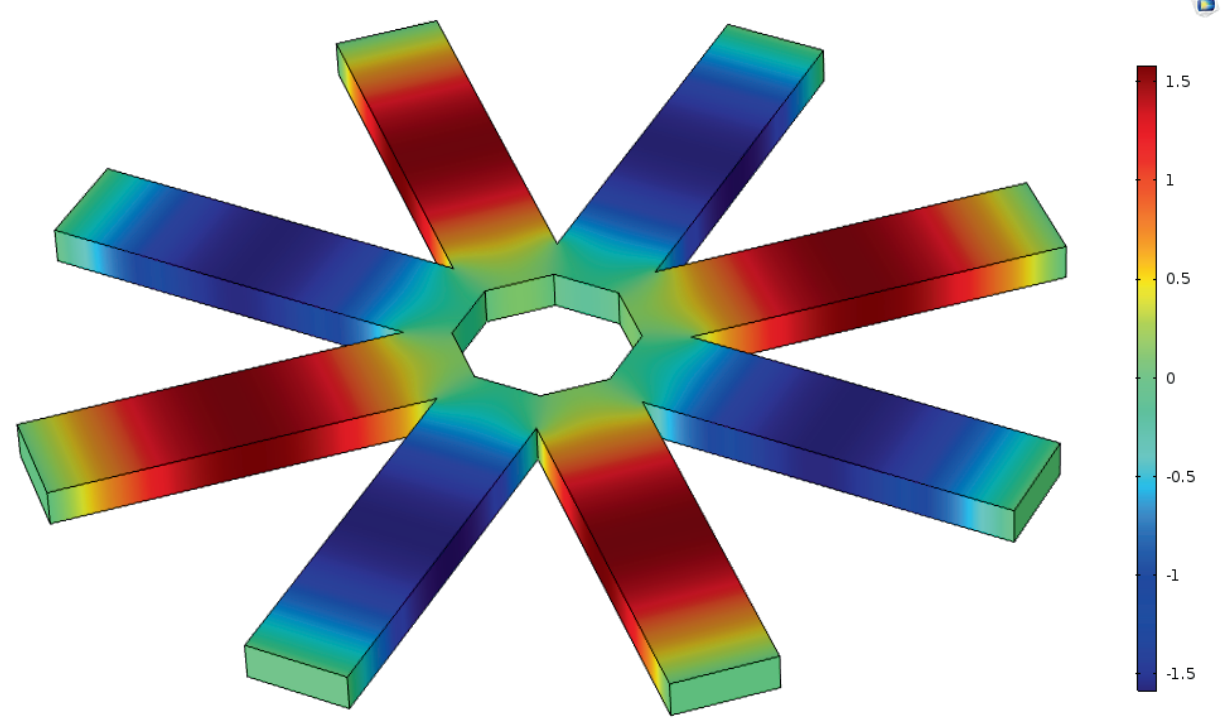

Figure 1.4.: Can-can eigen-mode in a generic multi-can setup

cient analysis between two cans in order to observe their acoustic interaction.

Papers by Rasheed et al. [29], [30], Baptista et al. [31] and Caldwell et al. [32] show the most recent development related to experimental studies concerning acoustic interaction between multi-tube combustor and single-stage axial turbines. Also the work of Deng et al. [33] investigates the interaction of a pulse detonation combustor integrated with a turbine hybrid system. Nicoud \& Poinsot [34] show an analytical method and the numerical approach to predict the thermoacoustic modes of industrial combustors. Several authors as Blimbaum et al. [35], Noiray \& Schuermans [36] and Bauerheim \& Poinsot [37] focused in their research on the study of thermoacoustic modes in annular combustion chambers through experimental, numerical or analytical methods.

In comparison to the previous papers, this part of the dissertation focuses on an accurate numerical analysis of the interaction between combustor and turbine, emphasizing also the acoustic relationship between two neighboring cans. The numerical analysis is performed for various 2D configurations compared with 3D geometries. This last part, related to the analysis and comparisons between 2D and 3D systems, distinguishes this work from the previous papers cited.

Another aspect of the current study that also introduces originality compared to the previous work, regards the comparisons performed between different 2D configurations presenting respectively straight and deflecting vanes. These configurations have been studied also taking into account various distances between the vanes and the wall connecting the two cans. This analysis delivers possible measures to suppress the cross-talk. 


\subsection{Thesis outline}

The introduction is followed by an overview of the theoretical basis necessary for the development of the current work in chapter 2. The numerical method used and the methods applied during the post-processing have been explained clearly.

The third chapter underlines the thermo-acoustical influence of the turbine on the combustor showing all $2 \mathrm{D}$ configurations analyzed in the first part of the study with comparisons between numerical results and analytical formulations.

The fourth section investigates the reflection coefficent behavior obtained for $2 \mathrm{D}$ and correspondent 3D geometries. The numerical results are compared to analytical formulae in order to estimate the validity of the numerical simulations for the chosen geometries.

The fifth chapter introduces the study concerning the coupling in can-annular combustors. The focus of this part is on the acoustic interaction between two cans. In this section comparisons have been presented between different 2D and 3D configurations. Also comparisons between different $2 \mathrm{D}$ configurations presenting respectively straight and deflecting vanes have been studied analyzing various distances between the vanes and the wall connecting the two cans. Measures to reduce the cross-talk have been carried out from this analysis.

The last part related to the general conclusions summarizes the contents and results of the previous chapters. It shows also an overview of the moving-mesh combustion solver developed in order to add the effects of the rotor to the 3D configurations analyzed before. It underlines how this feature for rotating meshes has been introduced and it presents as an outlook the necessity to perform numerical simulations with the 3D rotating mesh generated with the purpose to evaluate the acoustics of the 3D system. This method is however computationally very demanding. 
1. Introduction 


\section{Theoretical background - numerical method applied}

\subsection{Introduction}

This chapter describes the theoretical knowledge, on which this work is based. The main formulae cited and briefly explained are obtained from Lahiri [3], Kinsler, [38], Schade [39], Rienstra \& Hirschberg [40], Ehrenfried [41], Shapiro [42], Munjal [43], Watson [44], Ferziger \& Peric [45]. The numerical method applied has been shown with a short overview of the code used and the acoustic and cyclic boundary conditions set. The methods used and implemented for the post-processing are finally pointed out. 


\subsection{Propagation of waves in ducts}

\subsubsection{The conservation laws}

The Euler equations that represent the motion of a compressible and ideal fluid are shown briefly as a basis on which this current work is founded. They are known as conservation laws for mass, momentum and energy (Euler [46], Pierce [47], Schade [39]). The first equation for mass conservation gives a relation between the fluid with continuous properties and the density in Eq. (2.1):

$$
\frac{\partial \varrho}{\partial t}+\mathbf{u} \cdot \nabla \varrho+\varrho \nabla \cdot \mathbf{u}=0 .
$$

Eq. (2.1) can be expressed by using the total time derivative instead of the local time derivative as

$$
\frac{D \varrho}{D t}+\varrho(\nabla \cdot \mathbf{u})=0
$$

where the material derivative is given by the sum of the local derivative (first term) and the convective derivative as second term (Stokes [48], Batchelor [49] and Bird et al. [50]):

$$
\frac{D}{D t}=\frac{\partial}{\partial t}+\mathbf{u} \cdot \nabla
$$

In Eq. (2.4) the second equation for momentum conservation relates the velocity of the fluid to the pressure (Euler [46], Pierce [47]):

$$
\varrho\left(\frac{\partial \mathbf{u}}{\partial t}+\mathbf{u} \cdot \nabla \mathbf{u}\right)=-\nabla p
$$

where the gravity is neglected. The conservation of energy in Eq. (2.5) is represented by the enthalpy transport equation:

$$
\varrho \frac{D}{D t}\left(h+\frac{1}{2} \mathbf{u}^{2}\right)=\frac{\delta p}{\delta T}+-\nabla \cdot q
$$

with heat flux $q$ :

$$
q=-\frac{\lambda}{c_{p}} \nabla h-\left(\varrho D-\frac{\lambda}{c_{p}}\right) \sum_{i=1}^{\infty} h_{i} \nabla Y_{i}+q_{\text {radiation }},
$$

where $D$ is the effective diffusion coefficient, $c_{p}$ the heat capacity at constant pressure, and $\lambda$ the thermal conductivity. The set of equations is completed by the equation of state that for an ideal gas becomes (see Clapeyron [51] and Batchelor [49]):

$$
p=\varrho R T .
$$

The acoustic motion is evaluated as isentropic with the following relation between density and pressure (Batchelor [49], Pierce [47]):

$$
\left(\frac{\delta p}{\delta \varrho}\right)_{s}=c^{2}
$$

where $c$ is the speed of sound and $\frac{D s}{D t}=0$ (isentropic flow). 


\subsubsection{Linearization of Euler equations}

Pressure, velocity and density presented in the Euler equations can be expressed as the sum of their mean and fluctuating values in Eq. (2.12), Eq. (2.13) and Eq. (2.14):

$$
\begin{aligned}
& p=p_{0}+p^{\prime} \\
& u=u_{0}+u^{\prime} \\
& \varrho=\varrho_{0}+\varrho^{\prime} .
\end{aligned}
$$

The Euler equations can be linearized considering the fluctuating quantities smaller than the mean values:

$$
\begin{aligned}
\frac{p^{\prime}}{p_{0}} & \ll 1 \\
\frac{u^{\prime}}{u_{0}} & \ll 1 \\
\frac{\varrho^{\prime}}{\varrho_{0}} & \ll 1 .
\end{aligned}
$$

With this assumption, the second order derivatives in the Euler equations can be neglected leading to the approximate acoustic equations Eq. (2.15), Eq. (2.16) and Eq. (2.17) (Pierce [47], Goldstein [52]):

$$
\begin{gathered}
\frac{D \varrho^{\prime}}{D t}+\varrho_{0}\left(\nabla \cdot \mathbf{u}^{\prime}\right)=0 \\
\varrho_{0} \frac{D u^{\prime}}{D t}+\nabla p^{\prime}=0 \\
p^{\prime}=c^{2} \varrho^{\prime} .
\end{gathered}
$$

\subsubsection{Acoustic wave equation}

The complete derivation of the acoustic wave equation is presented in detail also in the work of Delfs [53]. The acoustic wave equation is obtained in few simple steps from Eq. (2.15), Eq. (2.16) and Eq. (2.17). The value of $\varrho^{\prime}$ obtained in Eq. (2.17) can be replaced in Eq. (2.15) and the second term of Eq. (2.15) can be written as the first term in Eq. (2.16) (Euler [46] and $[54])$ :

$$
\nabla^{2} p^{\prime}-\frac{1}{c^{2}} \frac{D^{2} p^{\prime}}{D t^{2}}=0
$$

where $\nabla^{2}$ is the Laplace operator. The following assumptions have been taken into account: homogeneous medium, ideal fluid, linear acoustics limited to small amplitude oscillations, isentropic relation between $\mathrm{p}$ and $\varrho$ and uniform flow. In case of stationary medium where 
$\mathbf{u}=0$, the total derivative in Eq. (2.18) can be substituted by the local derivative since the convective part becomes null (d'Alembert [55], Euler [46] and [56]):

$$
\nabla^{2} p^{\prime}-\frac{1}{c^{2}} \frac{\partial^{2} p^{\prime}}{\partial t^{2}}=0
$$

\subsubsection{Wave propagation in circular ducts}

In order to express the wave equation in Eq. (2.18) for a circular duct, cylindrical coordinates need to be used with axial coordinate $x$, radial coordinate $r$, and the circumferential one $\vartheta$. The three-dimensional wave equation becomes (Bronshtein [57]):

$$
\frac{\delta^{2} p^{\prime}}{\delta x^{2}}+\frac{1}{r} \frac{\delta}{\delta r}\left(r \frac{\delta p^{\prime}}{\delta r}\right)+\frac{1}{r^{2}} \frac{\delta^{2} p^{\prime}}{\delta \vartheta^{2}}-\frac{1}{c^{2}} \frac{D^{2} p^{\prime}}{D t^{2}}=0 .
$$

A general solution for the linear second-order partial differential equation in Eq. (2.20) is a sum of azimuthal and radial modes (Holste [58], Stahl [59], Neise [60], Duhamel [61], Rayleigh [62], Tyler \& Sofrin [63]):

$$
\hat{p}_{m n}(x, r, \vartheta, t)=\hat{p}_{m n}^{+} e^{-i k_{x, m n} x}+\hat{p}_{m n}^{-} e^{i k_{x, m n} x} J_{m n}\left(k_{r, m n} r\right)+Q_{m n} Y_{m n}\left(k_{r, m n} r\right) e^{i m \vartheta} e^{i \omega t}
$$

where $\left(\frac{\omega}{c}\right)^{2}=k_{x, m n}^{2}+k_{r, m n}^{2}$. The Eqn. (2.21) shows the modal solution where the modes are composed by spatial (axial, radial and circumferential) and temporal shapes. The spatial and temporal structures in axial and circumferential direction are sinusoidal, and the radial component is specified by Bessel functions. $J_{m}$ is the Bessel function of the first kind (Bessel function), $Y_{m n}$ is the Bessel function of the second kind (Neumann function) and $Q_{m n}$ is the n-th Eigenvalue of $Y_{m n}$ (Lahiri [3]). Each mode is described by circumferential order $m$ and radial order $n$, where $m$ and $n$ represent the nodal lines in circumferential and radial directions. In Fig. 2.1 the acoustic pressure in a duct is presented for four modal components. The circumferential and radial shapes are evident along the cross-section. Modes with higher circumferential order show a rotation around the $\mathrm{x}$-axis of the transversal structures. If $m=n=0$, no nodal lines are present in transverse direction, and the 1D sound field propagates only in x-direction (planar waves). The wave propagation in a cylindrical duct without a central hub $\left(Q_{m n}=0\right)$ with hard walls and uniform mean flow leads to a solution (Lahiri [3], Mason [64], Michalke [65], Morfey [66], Abramowitz [67]):

$$
\hat{p}_{m n}(x, r, \vartheta, t)=\left(\hat{p}_{m n}^{+} e^{-i k_{x, m n} x}+\hat{p}_{m n}^{-} e^{i k_{x, m n} x}\right) J_{m n}\left(k_{r, m n} r\right) e^{i m \vartheta} e^{i \omega t}
$$

with the derivatives of $J_{m}$ null at wall $J_{m n}^{\prime}\left(k_{r, m n} r\right)=0$ and with the radial number $k_{r, m n}=\frac{j_{m n}}{R}$ where $j_{m n}$ is the n-th Eigenvalue of $J_{m}$ for the hard-wall boundary conditions (Lahiri [3]).

\subsubsection{Cut-off frequency}

As explained in the previous section, in a cylindrical duct the wave equation in Eq. (2.20) has as a solution a sum of azimuthal and radial modes shown in Eq. (2.21) containing the axial wave number that defines the propagation of a mode in $\mathrm{x}$-direction:

$$
k_{x, m n}^{ \pm}=\frac{k}{1-M^{2}}\left(-M \pm \sqrt{1-\left(1-M^{2}\right)\left(\frac{j_{m n}}{k R}\right)^{2}}\right)
$$



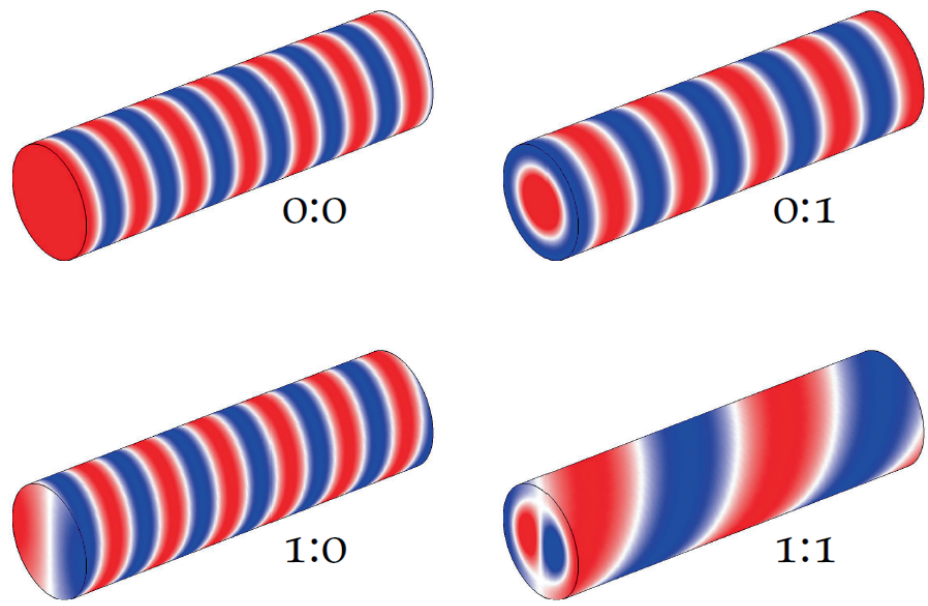

Figure 2.1.: Acoustic pressure in a cylindrical duct for four modal components (m:n), see Lahiri $[3]$

If the root is positive in Eq. (2.23), $k_{x, m n}^{ \pm}$is real and the mode propagates without any attenuation. When the root is negative, $k_{x, m n}^{ \pm}$is complex and the imaginary part is an attenuation coefficient. The propagation decays exponentially with axial distance and the mode can not propagate. This characteristic frequency determines the frequency where a mode is cut-off and when it can propagate:

$$
\omega_{c, m n}=\frac{j_{m n}}{2 \pi R} c \sqrt{1-M^{2}} .
$$

The cut-off limit is dependent on duct radius, mean Mach number, speed of sound and the Eigenvalue of the associated mode. In chapter 5 it will be show how the assumption of planar waves is not valid anymore beyond the cut-off value, proving an accurate post-processing of the results up to the cut-off limit using the tools implemented.

\subsubsection{Plane waves in a duct}

Plane waves are waves that can be described by one dimension only, here $x$. Their acoustic properties change with time and $x$, but they are constant along the planes normal to the direction of propagation. In these circumstances the acoustic wave equation in Eq. (2.18) becomes:

$$
\frac{\delta^{2} p^{\prime}}{\delta x^{2}}-\frac{1}{c^{2}} \frac{D^{2} p^{\prime}}{D t^{2}}=0
$$

A general solution is given by (see Munjal [43] and Pierce [47]):

$$
p^{\prime}(x, t)=F\left(t-\frac{x}{c}\right)+G\left(t+\frac{x}{c}\right)
$$


that describes two plane waves traveling in opposite direction with the speed c. A uniform mean flow in x-direction influences the traveling speed of the wave. If we assume the acoustic fluctuations as sinusoidal, $F$ and $G$ can be approximated by a Fourier series (Fourier [68]):

$$
F(t)=\sum_{\omega} \hat{p}_{\omega} e^{i \omega t}
$$

where $\hat{p}$ is the complex pressure with amplitude $|\hat{p}|$ and phase $\phi=\arg \hat{p}$. The acoustic pressure can be expressed in exponential notation where the relevant physical quantity (the real part) is taken (Munjal [43], Kinsler [38], Beranek [69], Morse [70]):

$$
p^{\prime}(x, t)=\left(\hat{p}^{+} e^{-i k x}+\hat{p}^{-} e^{i k x}\right) e^{i \omega t}
$$

where $k=\omega / c$ is the wave number (if the convective effect of the mean flow is added it becomes $k=\left(\frac{\omega / c}{1 \pm M}\right), \hat{p}^{+}$the complex pressure amplitude of the wave traveling in positive direction and $\hat{p}^{-}$in opposite direction. The Eq. (2.28) represents the temporal development and spatial distribution of the acoustic pressure of a $1 \mathrm{D}$, single frequency sound wave in a stationary medium, see Lahiri [3]. The wave number $k$ is related to the wavelength of a sinusoidal wave $\lambda=2 \pi / k$ defined as the spatial period of the wave, the distance over which the wave's shape repeats, and the inverse of the spatial frequency.

\subsubsection{Acoustic impedance}

The ratio of acoustic pressure to the associated particle speed in a medium is the specific acoustic impedance, see Kinsler [38] for details. In the case of duct acoustics, the acoustic impedance can be defined as the ratio between the Fourier transforms of the acoustic pressure and velocity depending on frequency:

$$
Z_{\omega}=\frac{\hat{p}_{\omega}}{\hat{u}_{\omega}}
$$

and for plane waves in free field the ratio becomes:

$$
Z_{0}=\varrho c .
$$

The product $\varrho c$ is releated to the characteristic property of the medium, see Kinsler [38]. Alternatively, the acoustic properties of a surface can be also defined by its complex reflection coefficient factor which describes the amount of an incident pressure wave $\hat{p}^{+}$that is reflected back from the boundary surface to form the $\hat{p}^{-}$wave traveling in the opposite direction (see Kinsler [38], Hanraths [6]):

$$
R_{\omega}=\frac{\hat{p}_{\omega}^{-}}{\hat{p}_{\omega}^{+}} .
$$

\subsection{Thermo-acoustic instabilities analysis}

In lean-premixed combustors in modern gas turbines, lean conditions and high energy density coupled with the compact flame region may lead to the development of oscillations. These 
kind of combustors do not have perforated liners and the damping characteristics are reduced. Thermoacoustic instabilities can be distinguished depending on the mode shape or the frequency range, see Kubis [10]. They can be divided into categories following the Siemens nomenclature:

- Low Frequency Dynamics (LFD)

- purely longitudinal modes

- low frequencies $f<50 \mathrm{~Hz}$

- Intermediate Frequency Dynamics (IFD)

- longitudinal modes

- intermediate frequencies $f=50-500 \mathrm{~Hz}$

- High Frequency Dynamics (HFD)

- modes with non-longitudinal components - three dimensional modes

- high frequencies $f>500 \mathrm{~Hz}$.

Low frequency oscillations are associated to purely longitudinal modes that can develop in all geometries, see Ghani [1], Dowling [71], Mugridge [72]. High frequency oscillations may consist of longitudinal, radial, and circumferential components. Typical frequencies of transverse modes are in the $\mathrm{kHz}$ range. High Frequency Dynamics oscillations are characterized by complex three dimensional mode shapes. Some modes as azimuthal can appear easily in annular combustors (Bourgouin et al. [73], Staffelbach et al. [74], Worth \& Dawson [75]). Transverse modes are excited in rectangular (Richecoeur et al. [76], Selle et al. [77]) and cylindrical combustion chambers (Schwing et al. [78], Zellhuber et al. [79]) but also appear in annular geometries, see Fig. 2.2. As it can be observed, longitudinal modes in rectangular or cylindrical ducts propagate in axial direction. Azimuthal modes can be excited in full annular chambers and in sectors as transverse perturbations. Transverse acoustic modes can appear in all types of combustion systems. Longitudinal modes have been mostly studied compared to transverse modes that can bring to very high pressure oscillations. The wavelength of longitudinal modes is larger compared to the diameter of the combustion chamber, justifying the assumption of the flame as compact $(\lambda \gg d)$. As said, transverse modes have higher frequencies and the wavelength can be of the same order as $d$. So the flame can not be considered as compact. For transverse modes it is necessary to compute shorter acoustic waves of the order of the channel width. The numerical effort is higher in case of transverse modes than for longitudinal modes, since they require more discretization points and higher precision numerical schemes.

\subsubsection{Control methods for thermo-acoustic instabilities}

There are two main options used to analyze and control thermo-acoustic instabilities. The first approach concerns the self-excitation of acoustic modes where the flame interacts directly with pressure waves (see Hernandez et al. [80], Hield et al. [81], Kostrzewa [82]). In this case the whole combustion system has to be simulated in order to capture the acoustic behavior in an accurate way. The second option is releated to forced response approach that has been used in this work. During the simulations a sound excitation has been provoked perturbing the flow field at the inlet of the combustion chamber. Multi-harmonic excitation with a 


\section{Longitudinal modes}

\section{Azimuthal modes}

Transverse modes

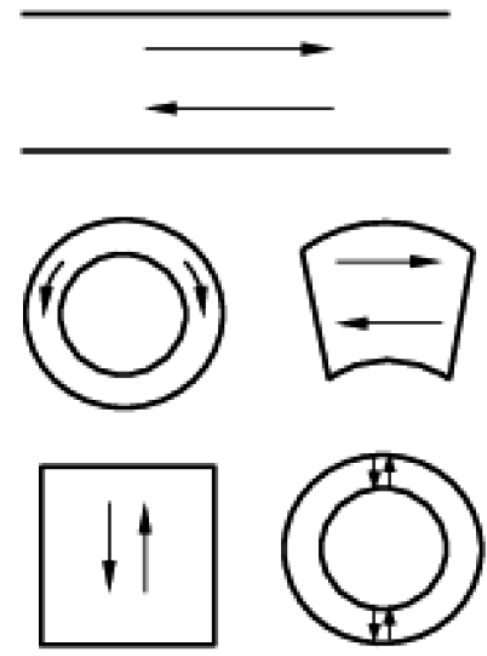

Figure 2.2.: Different modes depending on the geometry, see Ghani [1]

wide frequency range of $f=20-2000 \mathrm{~Hz}$ has been used at small amplitudes of less than $1 \%$ of the mean values to stay in the linear regime. In this method multiple simulations with different excitation frequencies need to be done but the computational power required is lower compared to the first method, since it requires a smaller CFD domain, covering only the relevant section.

\subsection{LES and CFD code applied}

In this dissertation the thermo-acoustic behavior is analyzed using numerical methods. Large Eddy Simulation (LES) has been applied. In this method the larger turbulent eddies are resolved numerically and just the smaller ones are modeled. This approach is more computationally expensive than Reynolds-averaged Navier-Stokes (RANS) where all turbulent eddies are modeled through an average of the Navier Stokes equations. Since LES solves all large unsteady flow structures, it is much more accurate than RANS for analysis of thermo-acoustic unsteady oscillations. LES simulations have been introduced first by Smagorinsky. The fluctuating quantities need to be resolved with sub grid models.

The choric Smagorinsky Model for compressible flows implemented in OPENFOAM represents one of the models applied during the study, see [83]. This Algebraic eddy viscosity Subgrid-scale (SGS) model is founded on the assumption that local equilibrium prevails. The formulae used are shown in Eq. (2.32):

$$
B=\frac{2}{3} k I-2 \nu_{s g s} \operatorname{dev} D
$$

where $D=\operatorname{symm} \nabla U, k$ from $\varrho D: B+\frac{c_{e} \varrho k^{\frac{3}{2}}}{\Delta}=0$ with $\Delta$ filter width, effective viscosity $\mu_{\text {sgs }}=c_{k} \varrho \sqrt{k} \Delta$, model coefficients $c_{e}=0.202$ and $c_{k}=0.02$. 
The LES One Equation Eddy Viscosity Model for compressible flows has been used as model in this work, see [84]. The Eddy viscosity SGS model is based on a modeled balance equation to simulate the behaviour of the kinetic energy $k$. The applied relations are presented in Eq. (2.33) and Eq. (2.34):

$$
\frac{d}{d t} \varrho k+d i v \varrho U k-d i v \mu_{e f f} \nabla k=-\varrho D: B-\frac{c_{e} \varrho k^{\frac{3}{2}}}{\Delta}
$$

and

$$
B=\frac{2}{3} k I-2 \nu_{\text {sgs }} \operatorname{dev} D
$$

where $D=\operatorname{symm} \nabla U, \nu_{s g s}=c_{k} \sqrt{k} \Delta$ with $\Delta$ as filter width, effective viscosity $\mu_{\text {eff }}=\mu_{\text {sgs }}+\mu$ with turbulence viscosity $\mu_{\text {sgs }}$, model coefficients $c_{e}=1.048$ and $c_{k}=0.094$. In parallel it has been decided to run also simulations with laminar flow, since the main aspect of the work is related to the acoustic behavior of the systems. No turbulence was modeled applying a simplication of the flow.

The numerical simulations performed in the current thesis are carried out mainly with the 2.1 version of the $\mathrm{C}++$ based, open-source CFD code OpenFOAM (Weller et al. [85], Greenshields [86] and [87], Holzmann [88]). As solver, sonicFOAm has been mostly used, since the geometries analyzed are transient and compressible. Few LES solvers have been applied based on transport equations solved using the semi-implicit PISO algorithm (Pressure Implicit with Splitting of Operators) introduced by Issa [89]. The momentum equation is solved as first to calculate the velocity field (see Jasak [90]). Between the different spatial and temporal discretization schemes present in OPENFOAM, $1^{\text {st }}$ order Euler and $2^{\text {nd }}$ order CrankNicolson schemes have been used in this work. This last one is more precise but less robust than the Euler scheme. The $C F L$ number is used to determine the time step based on the grid resolution and the speed of sound. To mantain the numerical solution stable a time step size of $\Delta t \approx 1 e-06 \mathrm{~s}$ is necessary to match the required condition $C F L<1$ in the numerical domain. The $C F L$ number is defined as:

$$
C F L=\frac{(|u|+c) \Delta t}{\Delta x}
$$

where $\Delta t$ is the time step, $\Delta x$ the cell size, $u$ the convective velocity and $c$ the speed of sound. For most of the configurations analyzed in this work the mesh grid used was quite coarse in order to resolve just the sound waves. Only the regions with higher Mach have been refined.

\subsection{Boundary conditions}

In the numerical simulations done during this study with a compressible subsonic flow, the standard setting of boundary conditions (von Neumann and Dirichlet) consists of a fixed inlet velocity and temperature as well as a fixed outlet pressure, in order to guarantee a solution of the system. This formulation with fixed absolute values for pressure and velocity flow leads to total reflection with $R=-1$, since the generated acoustic perturbations would be counterbalanced with a reflected wave of equal magnitude and reversed sign. Thus, acoustic energy cannot leave the simulation domain and will accumulate.

These unphysical reflected waves can provoke resonance and excitation phenomena within the 


\section{Theoretical background - numerical method applied}

numerical domain leading to inaccurate solutions. It is necessary to set as boundary values acoustic perturbations $p^{\prime}$ and $u^{\prime}$ to enforce a fixed mean values of $p$ and $u$. Also Yuen et al. [91] studied and analyzed the boundary reflection coefficient of acoustic systems. He pointed out that resonance frequencies might be reinforced with reflecting boundary conditions, resulting in high pressure peaks.

This issue can be solved by using Non-Reflecting Boundary Conditions (NRBC) as proposed by Poinsot \& Lele [92]. An ideally Non-Reflecting Boundary Condition is an accurate approximation for special flow conditions. The technical implementation and derivation of the NonReflecting Boundary Conditions applied in this work is described in Poinsot \& Lele [92] and Kaess et al. [93]. The system of partial differential equations constituting the Navier-Stokes equations is reformulated in terms of characteristic waves and their characteristic traveling velocity. Local One-Dimensional Inviscid (LODI)-relations have been defined describing the physical behavior of the boundary.

A relaxation factor has been introduced for the wave coming from outside of the domain, with the aim to define fixed mean pressure at the outlet or fixed mean velocity at the inlet. This factor is related to the time scale $\tau$ that must be as small as possible to avoid large deviations of the boundary conditions from the determined mean value (see Beck [4]). On the other hand, for small time scales the boundary conditions become fully reflective. Therefore it is necessary to define a compromise.

In Fig. 2.3 the measured reflection coefficient of the outlet boundary condition for a range of relaxation time scales is presented. The relaxation time scale is normalized with the oscillation frequency. To achieve a low reflection coefficient during the simulation the product of the lowest frequency of interest and the relaxation time scale should be $\tau \approx 10$. Since these NRBC can cause pressure drift, the modification of the time scale can reduce the problem but there is the need to find a compromise between reflection and allowed pressure drift. This fact led to the choice of $\tau=0.01 \mathrm{~s}$ for all simulations and NRBCs of this study.

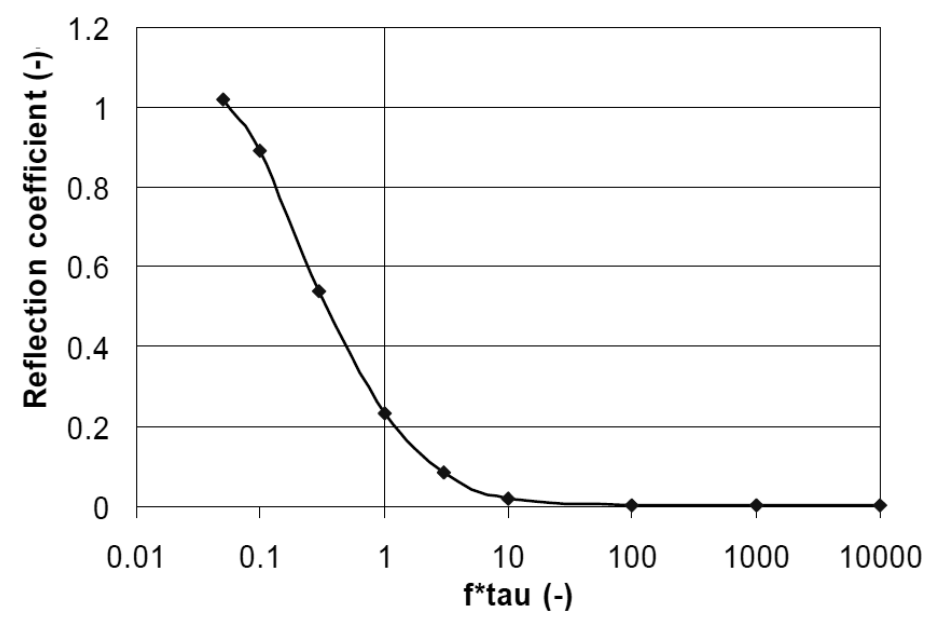

Figure 2.3.: Impact of the boundary condition time scale on the reflection coefficient, see Beck [4] 


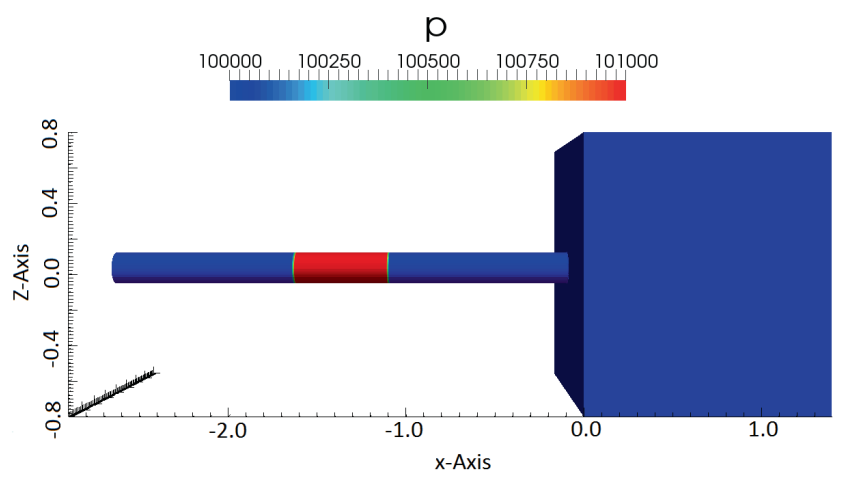

Figure 2.4.: Pipe geometry overview

\subsubsection{Evaluation of Non-Reflecting Boundary Conditions}

In order to evaluate and verify the Reflective Boundary Conditions (RBC) and NRBC, numerical simulations with the solver SONICFOAM have been done by studying a simplified pipe-baffle geometry with $d=200 \mathrm{~mm}$. Through the numerical tool SETFIELDS, the pressure $p=101000 \mathrm{~Pa}$ has been set along a chosen central box. Figure 2.4 shows the geometry analyzed pointing out the pressure pulse set at axial distance of about $x=-1.5 \mathrm{~m}$. Fig. 2.5 and Fig. 2.6 show the $\mathrm{x}$-velocity component and pressure distributions along the pipe geometry for both cases of RBC and NRBC analyzed.

Starting from the pipe region at $x=-1.5 \mathrm{~m}$ where an acoustic pressure pulse has been initiated with no velocity and high pressure, the wave propagates along the pipe in both directions: half of the pulse is directed to the inlet region and the other half part towards the outlet (baffle area). When the acoustic pulse reaches the pipe outlet at $x=0 \mathrm{~m}$, it finds an open end termination and it is subjected to physical reflection with phase turn. This phenomenon can be observed in Fig. 2.5(b) where the pulse reflected at the outlet presents opposite signs. The other half of the pulse travels towards the inlet hitting the boundaries set numerically as reflective. These numerical boundaries provoke a full reflection with $R=1$ without changing of phase. The velocity behavior for RBC has been studied in Fig. 2.5(a). In this case a similar trend obtained for the pressure values can be observed. The part of the wave traveling towards the outlet is also reflected back with same magnitude of the incoming pulse and with phase jumping. Anyway, the Fig. 2.5(a) shows with the same red color both incoming and reflected waves at $x=0 \mathrm{~m}$. This apparently incorrect behavior is due to the fact that the velocity is a vector, contrary to the pressure that is represented by an absolute value. For the velocity case, the reflected wave travels in opposite direction to the pulse propagation versus and it changes sign. This changing of sign compensates the turning of phase due to the open end termination and it results into the same positive sign of the incoming wave. The Fig. 2.6 presents the trend of velocity and pressure for NRBC set at inlet and outlet. In both Fig. 2.6(a) and Fig. 2.6(b) it is evident how the acoustic pulse propagates starting from $x=-1.5 \mathrm{~m}$ towards both inlet and outlet directions. At the inlet just 
2. Theoretical background - numerical method applied

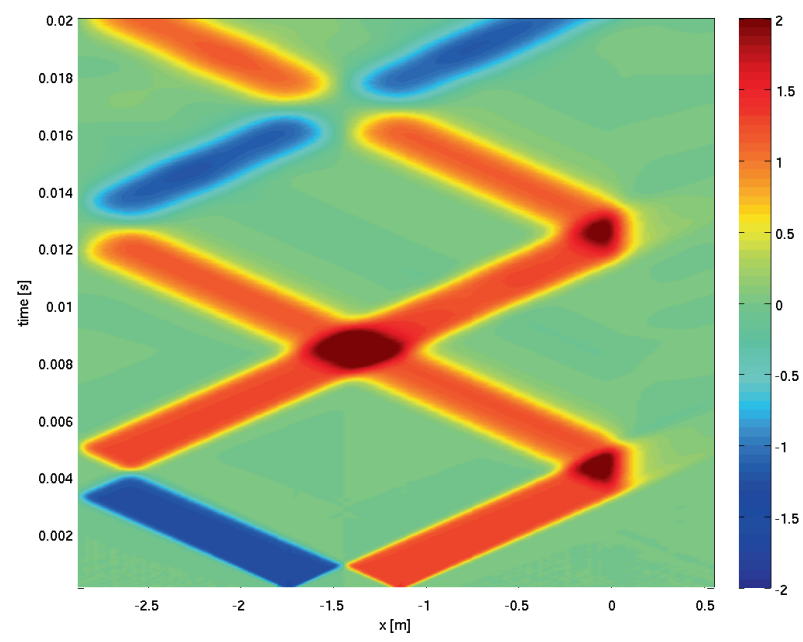

(a) $\mathrm{x}$-velocity component distribution in case of RBC

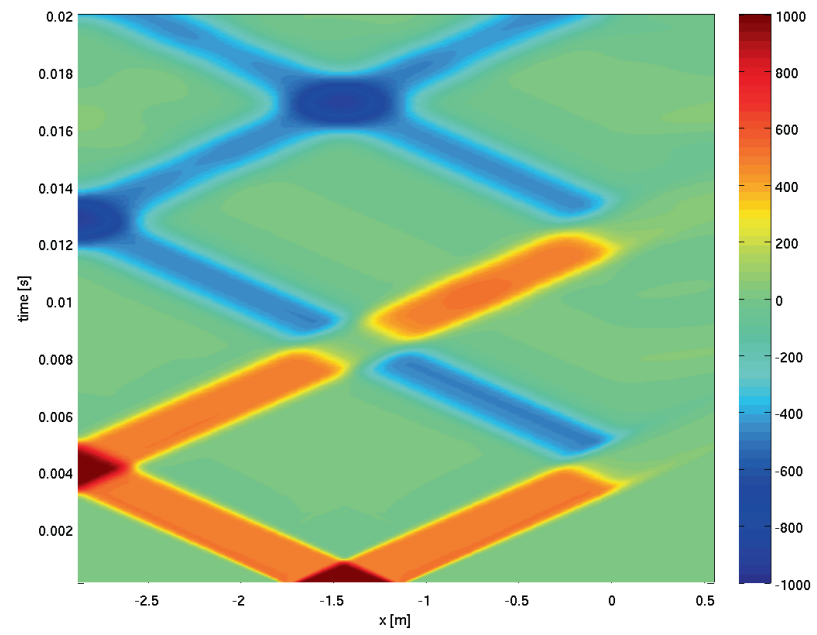

(b) Pressure perturbation in case of $\mathrm{RBC}$

Figure 2.5.: Distribution of velocity and pressure in case of RBC 


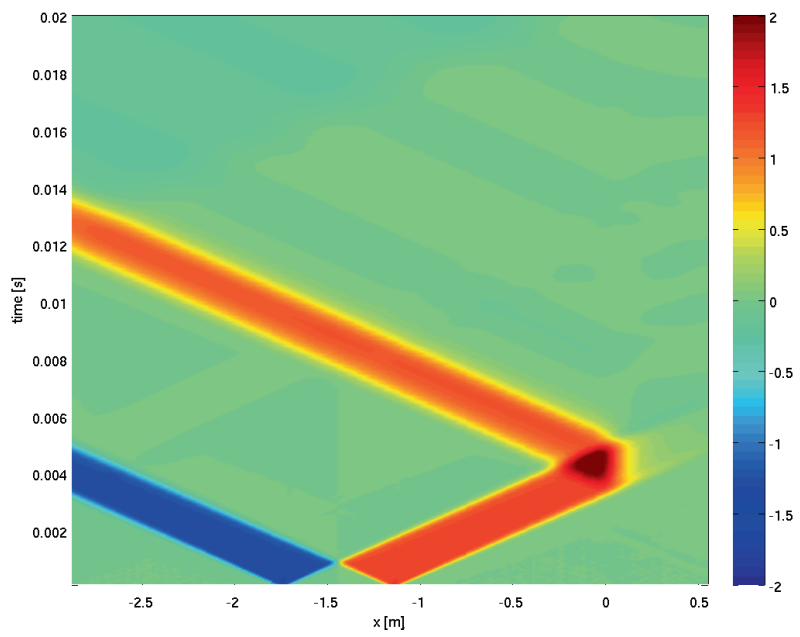

(a) $\mathrm{x}$-velocity component distribution in case of NRBC

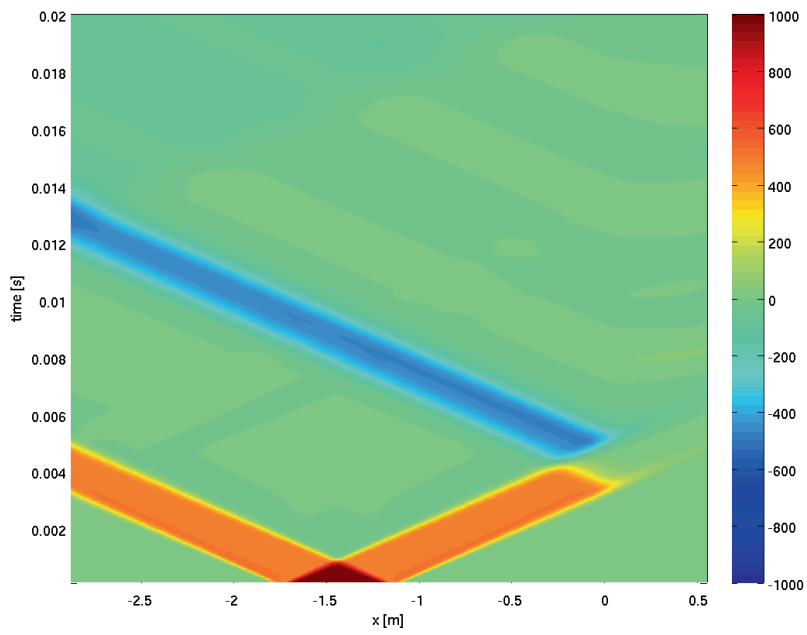

(b) Pressure distribution in case of NRBC

Figure 2.6.: Distribution of velocity and pressure in case of NRBC 


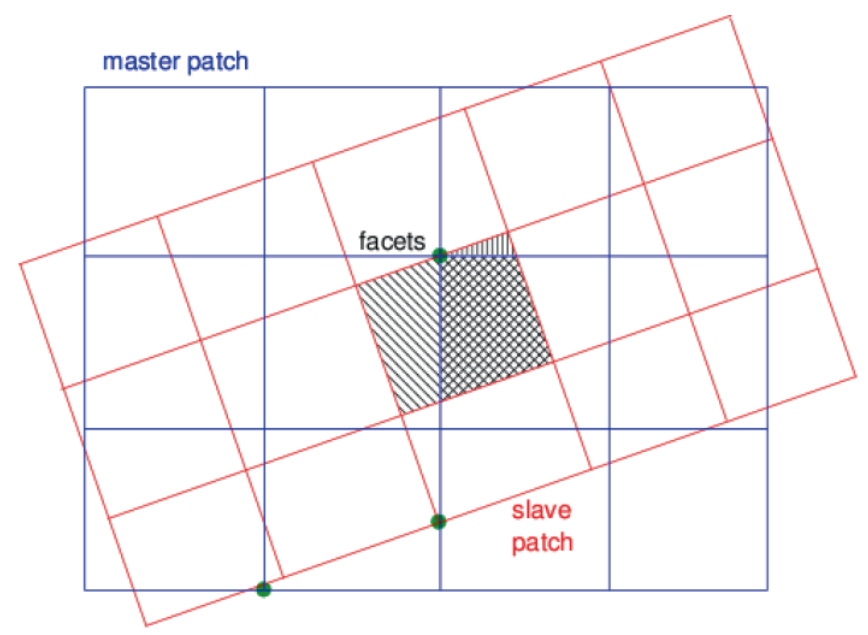

Figure 2.7.: Example of cyclic patches, see Jasak [5]

a small trascurable reflection is observed, proving the role and validity of the non-reflective boundaries. In Fig. 2.6 the reflection shown at the outlet of the pipe at $x=0 \mathrm{~m}$ is not due to the setting of NRBC but it is generated by the sound waves that hit the walls of the baffle geometry and are reflected back towards the pipe. As explained before, velocity and pressure show the same trend, but with opposite signs due to the fact that the velocity is represented by a vector. In the Appendix the numerical setting used in this work is described, taking as example Non-Reflective Boundary Conditions.

\subsubsection{Cyclic boundary conditions}

The numerical settings for cyclic boundary conditions have also been investigated during this work. The cyclic boundary conditions implemented in OPEnFOAM can be applied only in case of exact matching of the periodic patches with their neighboring periodic patches. Each pair of patches has to be totally planar with exact matching and same number of faces. For this reason for non-conformal patches an algorithm called AMI (Arbitrary Mesh Interface) has been implemented as next in OPENFOAM 2.1. AMI is comparable to the former GGI (General Grid Interface) presented in OpenFOAM 1.6 ext. In this case the interface between two cell regions is defined by a set of face zones and master and slave patches need to be specified on each side. The rotation of cell region is given in DynAmicMeshDict. For each time step, faces at the interface are cut into 'facets' (see Fig. 2.7), on which weighting factors need to be estimated with consistency and conservativeness conditions. The flow variables are transfered between master and slave patches through facets passing then to the following step, see Jasak [5] and Beaudoin \& Jasak [94]. Figure 2.7 presents as example the face intersection between master and slave patches. AMI are available for un-matched/nonconformal cyclic patches and sliding interfaces, on condition that both cyclic patches are almost planar. A differing number and area of faces is allowed, as long as they are partially 
covered by neighbouring connected faces. These settings have been investigated more in detail in the Appendix. An overview of different tutorials performed with cyclic boundary conditions during the study has been also presented in the Appendix.

\subsection{Post-processing - methods overview}

The harmonic signals studied in this dissertation are composed of a superposition of sinusoidal functions. The time series of sampling data along the sound path has been registered during the numerical calculations. Each microphone probe analyzed consists of a discrete time signal with $N$ sample elements for pressure, velocity and temperature. The idea is to transfer the original signal from the time domain into the frequency domain applying a Fourier transformation. This helps to simplify the acoustic analysis modifying the frequency range of interest. Furthermore, the acoustic boundary conditions are frequency-dependent, explaining their prevalent definition in frequency domain. The spectrum then obtained contains $N=2$ unique frequency informations (NYQUIST-SHANNON SAMPLING THEOREM) with $f_{\max }=1 /(2 d t)$. A SPECTRAL LEAKAGE could be generated with discontinuities at the end and beginning of the signal, resulting in data points in the frequency domain that do not match the originally excited frequencies exactly. This can be minimized by using a window function, that is multiplied to the original data, in order to ensure a smoother signal. This possible mismatch of the original excited frequencies depends on the frequency resolution, related to the simulation time. To adjust the number of samples $N$, ZERO PADDING is used, see Hanraths [6]. In this technique zeros are added to the end of the original signal changing the sample length without adding or removing information. This method achieves accurate results for discrete excitation also for low signal lengths. The main techniques applied in this work are shown in Fig. 2.8 as an example. At first, the pressure signal in the time domain is registered during the numerical simulations and then it is filtered by a Fourier transform adding the ZERO PADDING feature. Through the multi-microphone method the acoustic planar field is divided into the complex components traveling upstream $\hat{p}^{-}$or $G$ and downstream $\hat{p}^{+}$or $F$. In the last plot, the picks showing the amplitude of the frequencies excited at the inlet are clearly visible in the frequency domain.

Since the analysis of the pressure amplitudes of the upstream and downstream running sound waves is essential for the transmission and reflection coefficient estimation, different methods have been used in parallel for the post-processing. An overview of the methods applied is presented below.

\subsubsection{Two-microphone method}

The two-microphone method makes use of two microphones located at fixed positions. The excitation can be a random signal (containing all frequencies of interest) or a discrete frequency signal. The random noise signal is passed through a filter in order to hold only the desired frequency range. In the current study, a multi-harmonic excitation is set at the inlet of the geometries with a precise frequency range and the data sampled are recorded by microphones. The two-microphone method, as described by Seybert \& Ross, [95] and Munjal \& Doige [7], computes a CROSS POWER SPECTRAL DENSITY FUNCTION (cpsd) estimated in the 
2. Theoretical background - numerical method applied

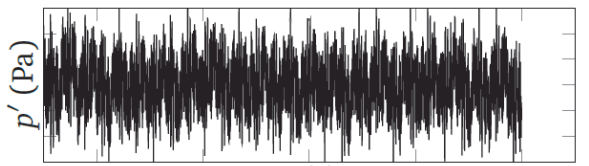

$t(\mathrm{~s})$

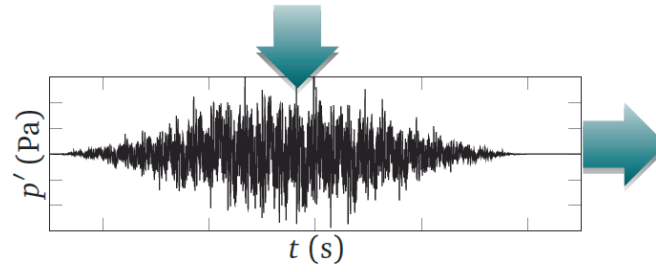

zero padded, filtered Fourier transform

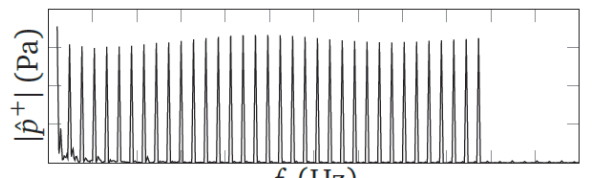

$f(\mathrm{~Hz})$

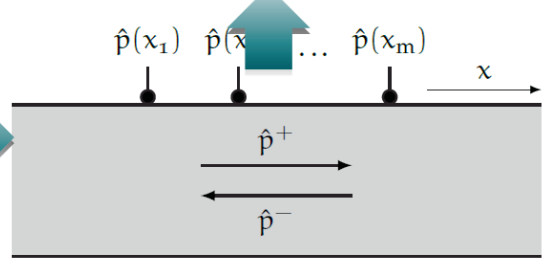

multi microphone wave decomposition

Figure 2.8.: Example of post-processing techniques applied, see Hanraths [6]

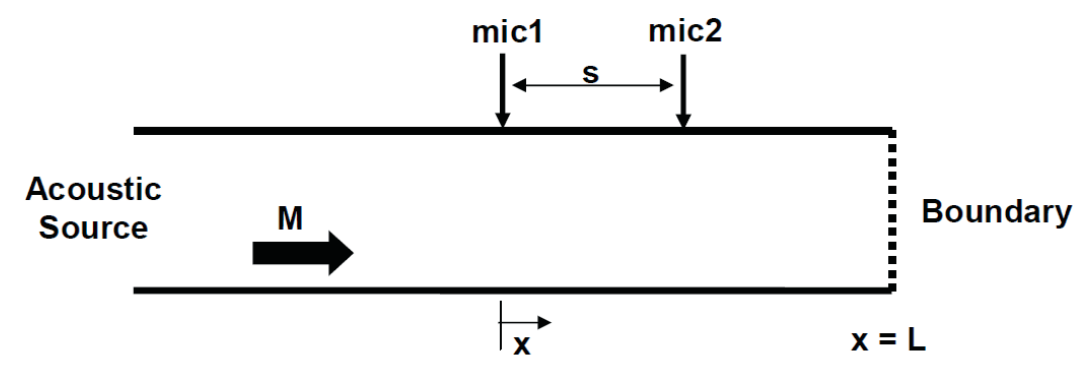

Figure 2.9.: Two Microphone Method with mean flow, see Munjal \& Doige [7]

frequency domain via Welch's method, see Lahiri [3]. This method calculates an average from overlapped windowed signal segments in order to smooth the spectrum. Through a window function more averages are taken, attenuating the signal at the beginning and end of each segment, compare with Lahiri [3]. The formula of the reflection coefficient used in the method is:

$$
R=\frac{H_{12} e^{-\beta M s}-e^{-\beta s}}{e^{\beta s}-H_{12} e^{-\beta M s}} e^{2 \beta L}
$$

where $H_{12}$ is the transfer function given by the ratio between the wave pressures at the two microphones, the wave number $k=2 \pi f / c\left(1-M^{2}\right)$ with $c$ speed of sound, $s$ is the distance between the two microphones, $L$ being the distance from the farthest microphone chosen to the boundary and $\beta=i k /\left(1-M^{2}\right)$. In Fig. 2.9 all parameters presented in the formula of the reflection coefficient applied in the study and discussed before have been shown.

The phase of $R$ depends on the parameter $L$. Therefore $L$ needs to be set consistently. In this work, the point where the geometries changes cross-section has been taken as boundary for the 2D analyzed nozzles. For the other 2D and 3D configurations, the leading edge of the vane has been taken as boundary. 


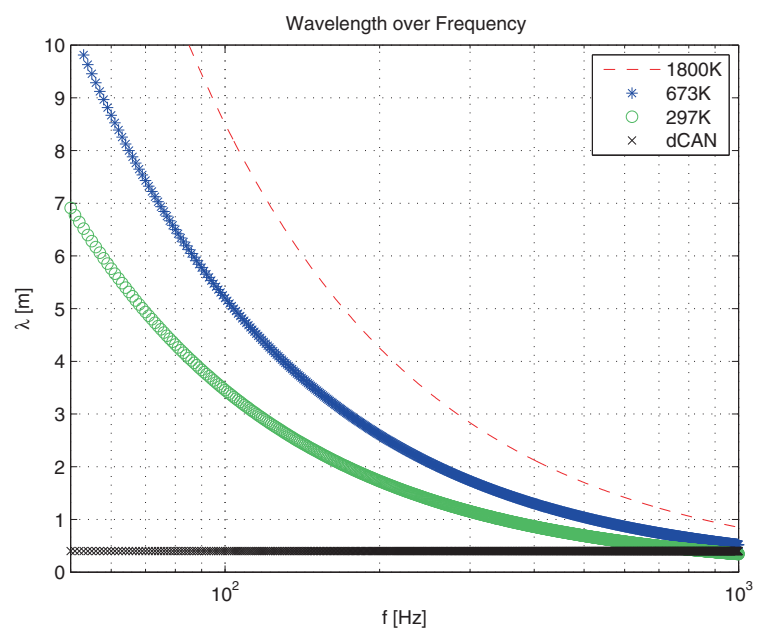

Figure 2.10.: Wave length over frequency for typical temperatures, can diameter for comparison

\subsubsection{Limits of the method}

The advantage of the method is based on the averaging and the lowered frequency resolution of the result leading usually to smoother curves. On the other hand the two-microphone method is sensitive to the microphone distance as observed during the validation study of the method, see Farisco et al. [96]. For microphone distances $s>\lambda / 2$ singularities can occur because half-waves fit between the microphones. Additionally the results quality can degrade quickly for contaminated signals due to the lack of redundancy. The two-microphone method only works for $1 \mathrm{D}$ acoustics (longitudinal waves) in ducts. For purely longitudianl waves the position of the mics on the cross-section has no influence on the result. Fig. 2.10 indicates the highest frequency for hot conditions $(1800 \mathrm{~K})$ that satifies $\lambda \gg d$.

\subsubsection{Amplitude evaluation method}

The following methods have been evaluated for the post-processing.

Scripts have been programmed in MATLAB analyzing the amplitudes of the waves traveling upstream and downstream. To obtain these two values the pressure and axial velocity at a single location have been analyzed. The formulae used for the complex values of pressure and velocity are presented in Eq.(2.37) and Eq.(2.38), compare with Ehrenfried [41]:

$$
\begin{gathered}
\hat{P}=A e^{i(\omega t-k x)}+B e^{i(\omega t+k x)} \\
\hat{U}=\frac{A}{\varrho c} e^{i(\omega t-k x)}-\frac{B}{\varrho c} e^{i(\omega t+k x)}
\end{gathered}
$$

where $\omega$ is the angular frequency in radians per second, $k$ is the wave number, $c$ the speed of sound, $x$ is the coordinate (1D), $A$ is the amplitude of the wave moving to the right and $B$ 
the wave traveling in the $-x$ direction. As following steps, it has been obtained:

$$
\begin{gathered}
\hat{U} \varrho c=A e^{i(\omega t-k x)}-B e^{i(\omega t+k x)} \\
A e^{i(\omega t-k x)}=\hat{U} \varrho c+B e^{i(\omega t+k x)} .
\end{gathered}
$$

Taking the Eq. (2.37) and replacing its term $A e^{i(\omega t-k x)}$ with Eq. (2.40):

$$
\begin{gathered}
\hat{P}=\hat{U} \varrho c+B e^{i(\omega t+k x)}+B e^{i(\omega t+k x)} \\
\hat{P}-\hat{U} \varrho c=2 B e^{i(\omega t+k x)} \\
B=\frac{\hat{P}-\hat{U} \varrho c}{2 e^{i(\omega t+k x)}} .
\end{gathered}
$$

Using Eq. (2.43) and replacing B in Eq. (2.37) and after the elimination of the term $e^{i(\omega t+k x)}$, it becomes:

$$
\begin{gathered}
\hat{P}=A e^{i(\omega t-k x)}+\frac{\hat{P}-\hat{U} \varrho c}{2} \\
A e^{i(\omega t-k x)}=+\frac{\hat{P}}{2}+\frac{\hat{U} \varrho c}{2} \\
A=\frac{\hat{P}+\hat{U} \varrho c}{2 e^{i(\omega t-k x)}} .
\end{gathered}
$$

Considering the time $t=0 \mathrm{~m}$ and an initial displacement of $x=0 \mathrm{~m}$, the two amplitudes $A$ and $B$ are calculated in a simplified way shown in Eq. (2.47) and Eq. (2.48):

$$
\begin{aligned}
& A=\frac{\hat{P}+\hat{U} \varrho c}{2} \\
& B=\frac{\hat{P}-\hat{U} \varrho c}{2} .
\end{aligned}
$$

A Fast Fourier Transform (FFT) has been applied to the pressure and axial velocity signals gained from sampling probes registered during the numerical simulations. Since the excitation signal had a comb-shaped spectrum, an average of the frequency domain results was necessary. The analyzed signal has been divided into windows, transformed using FFT and the amplitudes of each window have been averaged. This method presents less accuracy in comparison with the two-microphone method explained before. It shows a major presence of wiggles in the amplitude plots presenting a minor precision but it is easier to compute and applicable to $2 \mathrm{D}$ fields. 


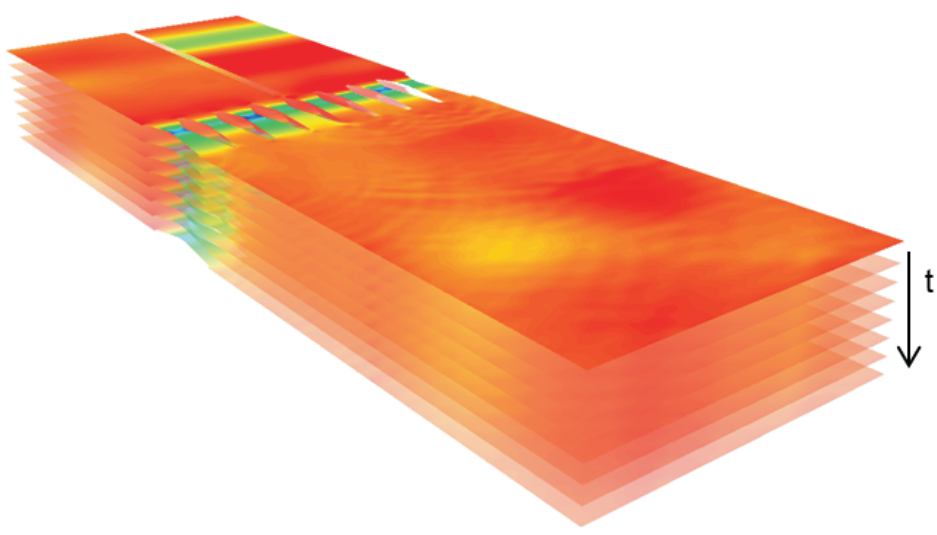

Figure 2.11.: Stack of VTK planes from OPENFOAM

\subsubsection{Multi-microphone method}

The multi-microphone method as described by Bothien et al. [97] and Lahiri [3] can be considered as an extension of the two-microphone method without Welch's method applied for averaging. It finds the complex amplitudes of the forward and backward traveling waves from the measured values of three (or more) microphones. From the one-dimensional wave equation, the plane wave acoustic field is decomposed into the upstream and downstream propagating part $G$ and $F$. Multiple axial pressure measurements are used in the current method in order to obtain an accurate approximation to the Riemann invariants in the frequency domain. In this case, the system of equations is overdetermined because the number of equations ( 3 or more) is larger than the number of unknowns (2) and cannot be solved by the application of conventional methods. The solution is a best fit in the least square sense by means of the Moore-Penrose pseudo-inverse function available in MATLAB (see Hanraths [6]). The values of the Riemann invariants have to be calculated using a non-linear optimization technique that search for the best fit of the input data. Since this method performs an average over several microphones, the flow noise is eliminated (Yang et al. [98]) and it overcomes the sensitivity to microphone spacing.

\section{DFT postprocessing: slowMotion}

With an FFT periodic signals can be detected in a simple way. This is usually done for a single location. This MATLAB script used for the field reconstruction does an FFT for a whole surface and generates nice moving images for selected frequencies. The moving is accomplished by rotating the phasor over a single period. The script reads a time series of Visualization ToolKit (VTK) surfaces and performs an FFT for each spacial point on the surface. In Fig. 2.11 a $2 \mathrm{D}$ configuration analyzed in chapter 5 is sliced into a time series of VTK surfaces. It can help to better understand und visualize the procedure performed in the script.

This allows a reconstruction of the acoustic or perturbation field for selected frequencies. The script generates diagrams with the Fourier coefficient magnitude on the surfaces and a wave 
reconstruction image for a fixed time. VTKs can be sampled by OPEnFOAM during run time. It is essential to sample enough time to cover the lowest desired analysis frequency $\left(f_{\min }=1 / t_{\text {sampled }}\right)$. The sampling frequency must be high enough to cover the highest frequency $\left(f_{\max }=(1 / d t) / 2\right)$. The results obtained from the tool are presented in chapter 5 . 


\section{Thermoacoustic Influence of the Turbine on the Combustor - validation of tool chain}

\subsection{Introduction}

This study of the combustor-turbine interaction is focused on the reflection coefficient analysis. The interface between the combustion system and the first turbine stage is the focus area of this part. The rotating components need to be included as outlook of this work.

Compressible Large Eddy Simulation (LES) resolving acoustics is applied based on the open source CFD code OpenFOAM. Different cases of increasing complexity are presented. The main idea is to begin the study based on simple geometries such as a convergent-divergent nozzle and two nozzles respectively convergent and divergent, to proceed with increased complexity by adding a vane section. These configurations are studied as basic generic tests in order to validate analytical formulae and to test the CFD methods applied.

Calculations with acoustic excitation and Non-Reflecting Boundary Conditions (NRBC) at the computational inlet and outlet domains are carried out to verify the plausibility of the 
acoustic set up. A forced response approach is applied provoking a wave excitation at the inlet of the combustion chamber. Multi-harmonic excitation with small amplitudes is used to stay in the linear range. The post-processing for all cases is performed using the two-microphone method. The outcomes are pointed out in the last part of the chapter.

\subsection{Investigated configurations}

The cases selected for the analysis are depicted in Fig. 3.1 and described below in more detail. They have been chosen because they represent typical elements found in gas turbines.

\section{Convergent and divergent nozzles}

The convergent and divergent nozzles shown in Fig. 3.1(a) and 3.1(b) represent typical flow elements found at the inlet and outlet of a combustion chamber. For these parts the analytical solutions for the reflection coefficient at low frequencies are well known. The convergent and divergent elements will be called $\mathrm{CN}$ and DN through the work.

\section{Convergent-divergent nozzle}

A convergent-divergent nozzle as shown in Fig. 3.1(c) can be often found as acoustic termination in combustion test rigs where no turbine stages can be used. Its acoustic properties need a frequency-dependent description since there is no reflection at the low frequency limit. A one-dimensional description can be applied up to high frequencies. The case will be called CDN.

\section{D stator vane}

The 2D stator vane represents well a realistic stator stage if no gradients are assumed along the airfoil span. The limitation to $2 \mathrm{D}$ reduces the computational time significantly. The flow element combines the aforementioned cases. It has equal cross-sections before and after the profile, but increasingly different thermodynamic conditions in case of increasing flow. The case will be named $2 \mathrm{dV}$ and is shown in Fig. 3.1(d).

\subsection{Numerical method}

\subsubsection{Solver and boundary conditions}

For all simulations presented in section 3.6 compressible LES has been applied based on the open source CFD code OPENFOAM. A compressible solver SONICFOAM with second order discretization in space and time has been used to compute the solutions. The $2 \mathrm{D}$ cases have been performed with Non-Reflecting Boundary Conditions (NRBC) set at the inlet and outlet of each geometry. The duration of the numerical calculations is up to $t=2 \mathrm{~s}$ real time.

LES models have been presented in section 2.4. The role of NRBC is to ensure the flow 


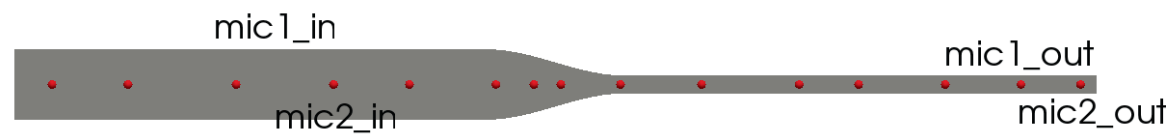

(a) Case CN: Convergent nozzle

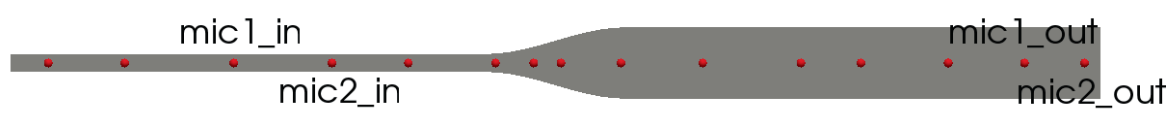

(b) Case DN: Divergent nozzle

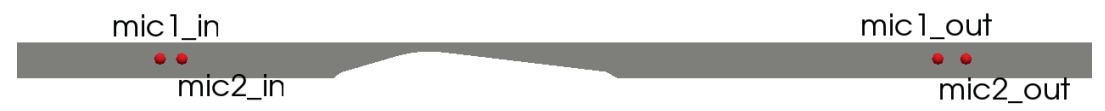

(c) Case CDN: Convergent-Divergent Nozzle

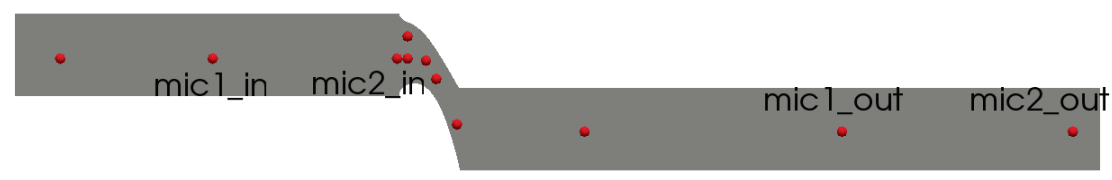

(d) Case 2dV: 2D stator vane

Figure 3.1.: Nozzle geometries analyzed in 2D. The red marks depict the microphones used for the two-microphone method 


\begin{tabular}{lll}
\hline$U_{\text {inlet }}$ & Mach $_{\text {inlet }}$ & Mach $_{\text {throat }}$ \\
\hline 0 & 0.001 & 0.02 \\
50 & 0.06 & 0.24 \\
76 & 0.09 & 0.39 \\
95 & 0.1 & 0.49 \\
\hline
\end{tabular}

Table 3.1.: Convergent-divergent nozzle: Flow conditions

\begin{tabular}{lll}
\hline$U_{\text {inlet }}$ & Mach $_{\text {inlet }}$ & Mach $_{\text {outlet }}$ \\
\hline 0 & 0.002 & 0.002 \\
50 & 0.07 & 0.25 \\
76 & 0.12 & 0.40 \\
95 & 0.14 & 0.51 \\
\hline
\end{tabular}

Table 3.2.: Convergent nozzle: Flow conditions

conditions set, removing unphysical effects of wave reflection. The derivation of the NRBC is performed using Local One-Dimensional Inviscid (LODI) relations to obtain approximate values for the wave-amplitude variations in terms of the primitive flow variables as shown in the papers of Polifke \& Wall [99], Polifke et al. [100] and Widenhorn et al. [101]. The walls of the CDN, CN and DN have been set as slip walls. For the $2 \mathrm{dV}$ case with realistic vanes deflecting the flow, the periodicity has been taken into account.

The cases have been analyzed setting at the inlet different mass flows $\dot{m}$ resulting in different Mach numbers. Table 3.1, 3.2, 3.3, 3.4 present the main values considered for the different configurations. Depending on the velocity set at the inlet, the operating conditions have been varied maintaining anyway not choked flow in the throat. The simplest case represents the condition of no flow, followed by gradually increased Mach numbers in the inlet $(M<1$ in the throat). The numerical behavior of the Mach numbers has been analyzed for all cases and verified with the analytical formulations used to calculate the Mach number for compressible flows.

\subsubsection{Meshing}

Considering the CDN, the mesh generated consists of 15106 cells. For the two CN and DN, the meshes present in both cases 1313 cells. For the $2 \mathrm{dV}$ test case the mesh consists of about 24400 cells. The tool used to generate the meshes for all 2D cases was BLOCKMESH. 


\begin{tabular}{lll}
\hline$U_{\text {inlet }}$ & Mach $_{\text {inlet }}$ & Mach $_{\text {outlet }}$ \\
\hline 0 & 0 & 0 \\
50 & 0.033 & 0.014 \\
76 & 0.051 & 0.023 \\
95 & 0.061 & 0.028 \\
\hline
\end{tabular}

Table 3.3.: Divergent nozzle: Flow conditions

\begin{tabular}{lll}
\hline$U_{\text {inlet }}$ & Mach $_{\text {inlet }}$ & Mach $_{\text {throat }}$ \\
\hline 0 & 0.006 & 0.02 \\
76 & 0.08 & 0.20 \\
95 & 0.11 & 0.241 \\
120 & 0.14 & 0.58 \\
\hline
\end{tabular}

Table 3.4.: 2D stator vane: Flow conditions

\subsection{Two-microphone method}

As described previously, a forced response approach has been used during the simulations. An excitation has been imposed at the inlet of the CDN, CN, DN and $2 \mathrm{dV}$ geometries with a wide frequencies range $f=20-2000 \mathrm{~Hz}$. A series of sampling data have been obtained from the calculations. The location of the probes registered is presented in Fig. 3.1. The sampling data are used for the post-processing of the cases performed through the two-microphone method described by Munjal \& Doige [7]. The formula of the reflection coefficient used in the method is shown in Eq. (2.36).

\subsubsection{Two-microphone method validation}

For the validation of the two-microphone method the microphone signals were generated analytically for a straight duct and the parameters $R_{v a l}=0.5, M=0.3$ at a speed of sound of $c=800 \mathrm{~m} / \mathrm{s}$. Test signals consisted of multiple sine waves with $f<2000 \mathrm{~Hz}$. The results obtained are presented in Fig. 3.2. The microphone distance $s$ has been chosen deliberately below and above the criterion of $s<\lambda / 2$. For higher distances half-waves fit between the microphones causing singularities in the method. As shown in Fig. 3.2(b) the results deviate for the singular frequencies. The correct choice of $s$ leads to correct results as depicted in Fig. 3.2(a). 

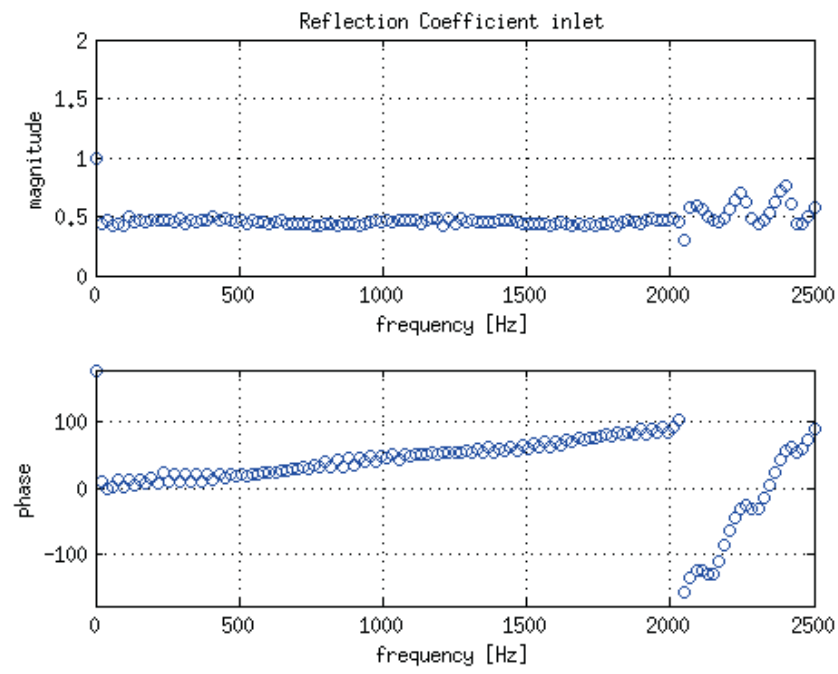

(a) $R_{\text {val }}=0.5, M=0.3, s=0.02 \mathrm{~m}$
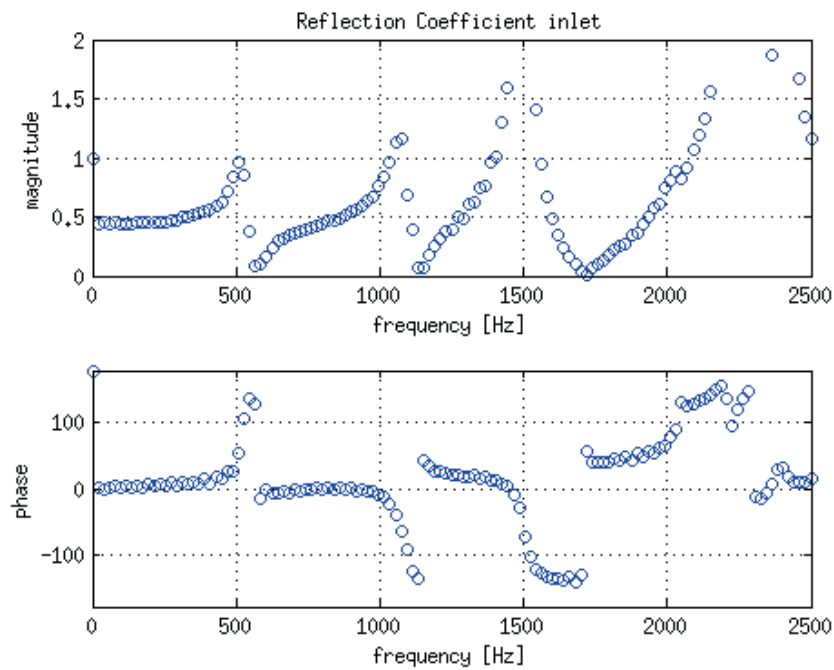

(b) $R_{\text {val }}=0.5, M=0.3, s=0.7 \mathrm{~m}$

Figure 3.2.: Two-microphone method validation performed choosing two different microphone distances $s$ below and above the criterion $s<\lambda / 2$ 


\subsection{Analytical solutions}

To access the quality of the CFD results a comparison with analytical results is made where available.

\subsubsection{Low frequency approximation}

Combustion noise has been studied by Marble \& Candel [19] using an analytical method based on compact nozzle hypothesis, valid only in the limit of low frequencies. In this assumption the characteristic wavelength $\lambda$ of waves is larger compared to the nozzle length $L, \lambda \gg L$. For example, the results obtained for the convergent and divergent nozzles shown in Fig. 3.4(a) and Fig. 3.6(a), prove the validity of this assumption for the frequencies up to about $500 \mathrm{~Hz}$ where $\lambda \approx 20 L$. The book of Ehrenfried [41] presents the formula of the reflection coefficient for a cross-section change geometry extended to flow without any references to the frequencies. The numerical results for $R$ at the inlet in the two convergent and divergent nozzles have been studied in comparison with this analytical formula shown in Eq. (3.1):

$$
R_{1}=\frac{\frac{\varrho_{1} S_{1}}{c_{1}}-\frac{\varrho_{2} S_{2}}{c_{2}}}{\frac{\varrho_{1} S_{1}}{c_{1}}+\frac{\varrho_{2} S_{2}}{c_{2}}} \frac{1+M_{1}}{1-M_{1}}
$$

in order to analyze the low frequencies range also in the numerical cases.

\section{Transfer matrix method}

The application of the low frequency approximation from Eqn. (3.1) to ducts with equal inlet and outlet cross-section results in the trivial solutions of full transmission. To study the effect of the cross-section change a different approach is used. This method is valid for the assumption $\lambda \gg d$ where $d$ represents the diameter of the geometry. To obtain the frequency dependent impedance and reflection coefficient of a duct with slowly varying cross-section the transfer matrix method can be used. In this method the geometry is sliced in a sequence of elements with constant cross-section for which the transfer matrix is known. The transfer matrix relates the acoustic pressure and velocity at the inlet and outlet of each duct element. The relation reads Mechel [102]:

$$
\left[\begin{array}{l}
p_{1} \\
u_{1}
\end{array}\right]=T\left[\begin{array}{l}
p_{2} \\
u_{2}
\end{array}\right]
$$

with

$$
T=e^{-i M k_{c} L}\left[\begin{array}{cc}
\cos \left(k_{c} L\right) & i(\varrho c / S) \sin \left(k_{c} L\right) \\
i S /(\varrho c) \sin \left(k_{c} L\right) & \cos \left(k_{c} L\right)
\end{array}\right]
$$

and $L$ being the length, $S$ the cross-section, and $k_{c}=k /\left(1-M^{2}\right)$ the wave number in each duct element.

The response of the whole geometry is obtained by multiplying the transfer matrices of all duct elements

$$
T=T_{1} \cdot T_{2} \cdots T_{n}
$$


The termination is set to the free field impedance representing anechoic conditions or an infinite continuation of the duct. Finally the reflection coefficient can be calculated with

$$
R=\frac{Z-Z_{0}}{Z+Z_{0}}
$$

where $Z_{0}$ is the local free field impedance and $Z$ the impedance of the duct calculated from Eq. (3.4). A formulation for elements with compressible flow has been proposed for example by Miles [103] or Dokumaci [104], but is not treated here.

\subsection{Results}

Following the discussion in the previous sections, the reflection coefficient obtained from CFD simulations is analyzed for all configurations. Comparisons with analytical results are shown. For the two-dimensional configurations, exemplary results with and without mean flow are discussed.

The results show the reflection coefficient $R_{1}$ behavior calculated in the point located in the middle of the duct before the variation of the cross-section. The point taken as reference for the $2 \mathrm{D}$ stator vane is placed at the leading edge of the vane.

In the ideal case, the result is not influenced by unphysical waves reflected by the nonreflecting boundary and it represents only the physics of the geometry. To verify the correct operation of the boundary termination, the reflection coefficient called $R_{2}$ is also analyzed. The ideal value is $R_{2}=0$. The following diagrams shown in this section include the nonexcited frequency range beyond $f>2000 \mathrm{~Hz}$. This part of the results is presented only for reference. Depending on the flow, two types of analytical solutions are used for comparison. For no flow, the transfer matrix method based on equation (3.3) was preferred. Since the transfer matrix method was not yet implemented for cases with higher Mach numbers, the low frequency approximation was used in situation of flow as reference since its validity is proven in literature by Marble \& Candel [19], Lamarque \& Poinsot [24] and Ehrenfried [41].

\subsubsection{Convergent nozzle}

The numerical results for the convergent nozzle $\mathrm{CN}$ are shown in Fig. 3.3 and 3.4.

In case of no flow, see Fig. 3.3(a), the magnitude of $R_{1}$ is presented as a function of frequency. The curve obtained from the numerics follows the same trend compared to the analytical frequency-dependent formula, showing a flat behavior around $R_{1}=0.6$ with decreasing values starting from $f=700 \mathrm{~Hz}$ until the maximum limit of the excitation set at $f=2000 \mathrm{~Hz}$. As expected, the plot shows a random behavior of $R_{1}$ for $f>2000 \mathrm{~Hz}$, where no forcing was applied.

The two-microphone method used is sensitive to the microphone distance $s$ between the microphones as observed during the validation study of the method shown before. So particular attention needs to be given to the choice of the parameter $s$.

Concerning the phase of $R$, this is by definition dependent on the parameter $L$ representing the distance from the farthest microphone chosen to the boundary. For the geometries studied without vanes the boundary is located at the first change of cross-section observed after the 

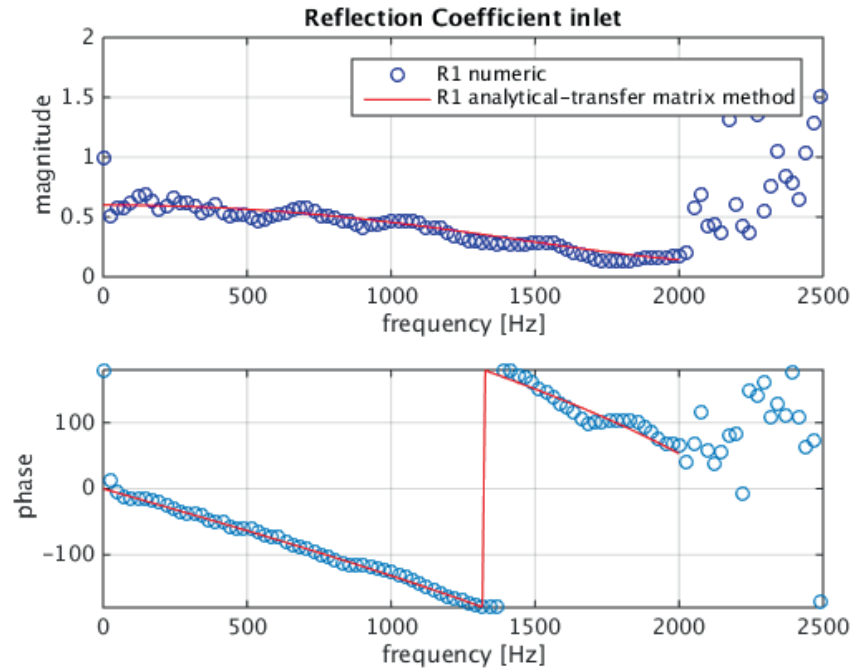

(a) $R_{1}$ - no flow
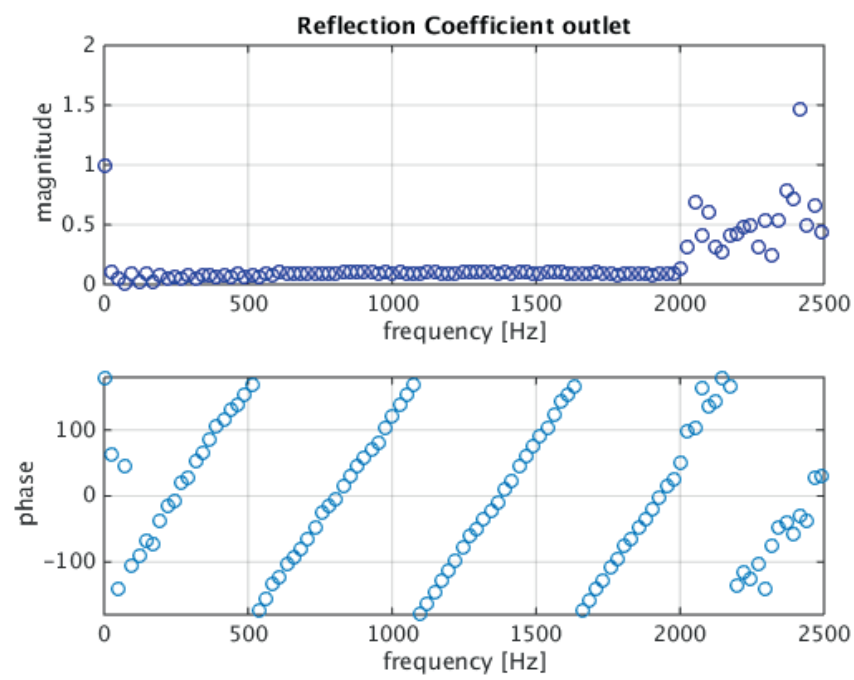

(b) $R_{2}$

Figure 3.3.: Reflection coefficient for convergent nozzle: no flow conditions 

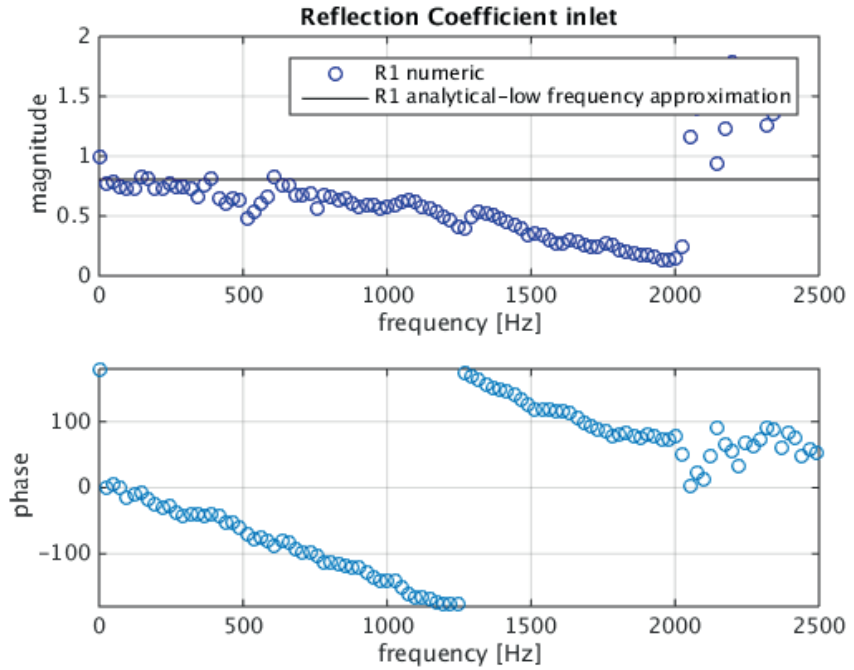

(a) $R_{1}-M_{\text {inlet }}=0.14, M_{\text {outlet }}=0.51$
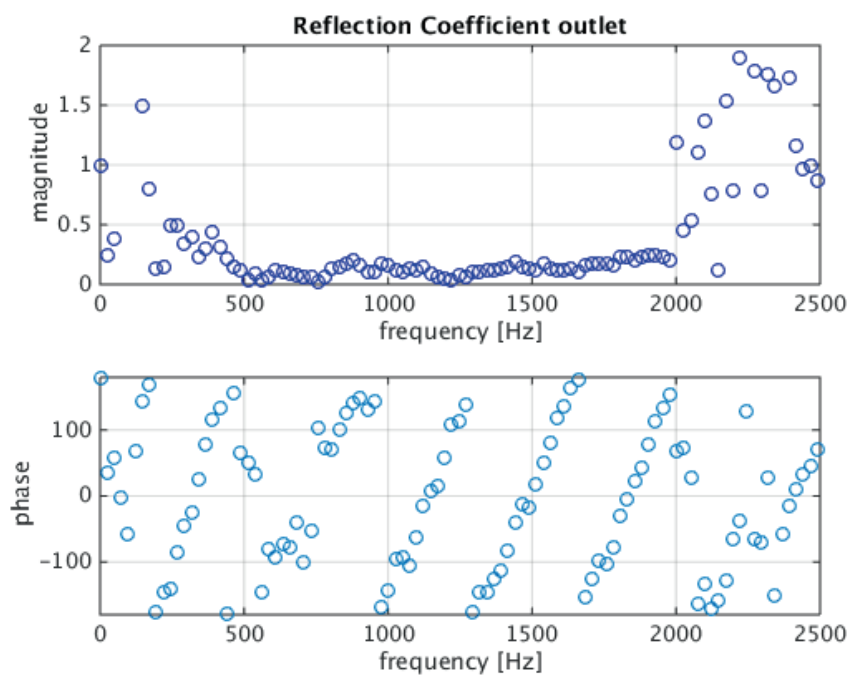

(b) $R_{2}$

Figure 3.4.: Reflection coefficient for convergent nozzle: flow conditions 
inlet. For the 2D case with deflecting vane, the leading edge of the vane has been taken as boundary.

The magnitude of $R_{2}$ at the outlet in case of no flow (Fig. 3.3(b)) maintains a smooth trend with constant value 0 as expected. This behavior confirms the validity of the NRBC applied. The case with flow $\left(M_{\text {inlet }}=0.14\right)$ has been compared with the low frequency approximation. The amplitude of $R$ at the inlet shown in Fig. 3.4(a) shows an accurate agreement between the numerical behavior and the analytical formulation. It presents a quite flat trend with higher values compared to no flow case around $R_{1}=0.8$ until $f=700 \mathrm{~Hz}$. Beyond this frequency the reflection curve starts to deviate from the analytical formula with a decreasing behavior reaching almost $R_{1}=0$ for $f=2000 \mathrm{~Hz}$.

The reflection at the outlet in Fig. 3.4(b), shows an overall quite constant behavior with values around zero for $f>400 \mathrm{~Hz}$. It shows reflection for low frequencies until about $f=400 \mathrm{~Hz}$, due to low reliability of the NRBC in this frequency range, related to the choice of the relaxation factor explained in section 2.5. The phase for the reflection coefficient at the outlet shows a different behavior compared with the $R_{1}$ phase, since $L$ has been modified with the choice of two other microphones at the outlet.

\subsubsection{Divergent nozzle}

The results obtained for the divergent nozzle DN are shown in Fig. 3.5 for no flow. Figure 3.5(a) presents the amplitude of the reflection coefficient evaluated at the inlet as a function of frequency. The numerical curve appears similar to the convergent case with same trend and values around $R=0.6$ that are decreasing until $f=2000 \mathrm{~Hz}$. The numerical results validate the analytical formula pointing out an accurate agreement within the whole frequency range analyzed. The phase shows also the same behavior compared to the formula. In Fig. 3.5(b) no reflection is shown at the outlet proving the validity of the NRBC.

As summary, the numerical results concerning the reflection coefficient magnitude and phase, present good agreement with the analytical curve.

In Fig. 3.6 the numerical solution with $\left(M_{\text {inlet }}=0.06\right)$ is compared with the low frequency approximation.

Figure 3.6(a) shows a constant trend with values of $R_{1}=0.7$ that are decreasing then for $f>700 \mathrm{~Hz}$ and deviating from the analytical solution. Also with flow $R_{2}$ in Fig. 3.6(b) fulfills the expectations.

\subsubsection{Convergent-divergent nozzle}

In Fig. 3.7 and 3.8 the numerical results for the convergent-divergent nozzle (CDN) are presented.

Concerning the diagram showing the behavior of $R_{1}$ without flow in Fig. 3.7(a), the numerical values for both magnitude and phase underline a reliable trend in agreement to the analytical frequency-dependent formula for the analyzed frequency range $f=20-2000 \mathrm{~Hz}$. Analyzing $R_{2}$ at the outlet in Fig. 3.7(b), the flat behavior of the curve confirms the reliability of the NRBC also in this case.

In case of reflection at the inlet with flow $\left(M_{\text {inlet }}=0.1\right)$ in Fig. 3.8(a), similar results have 

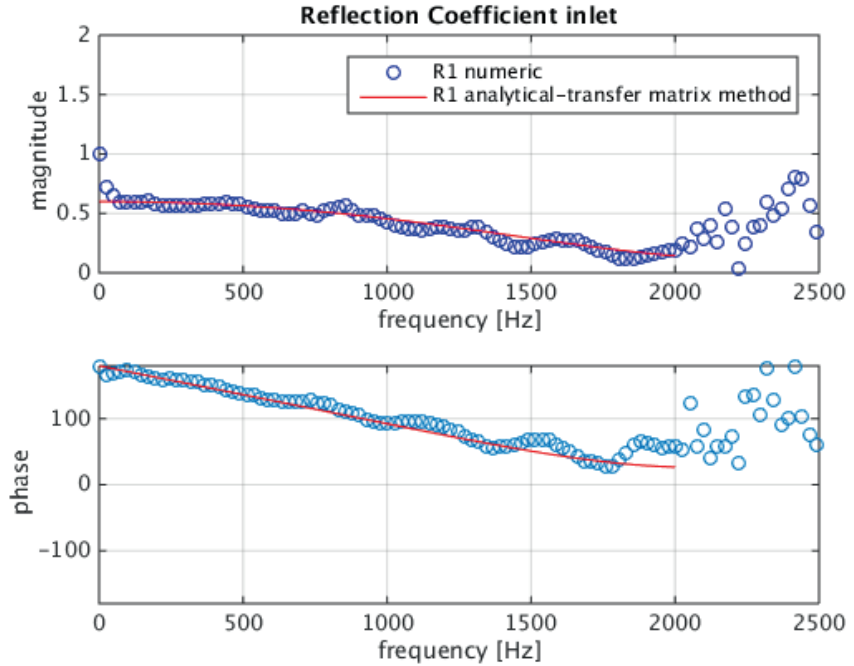

(a) $R_{1}$ - no flow
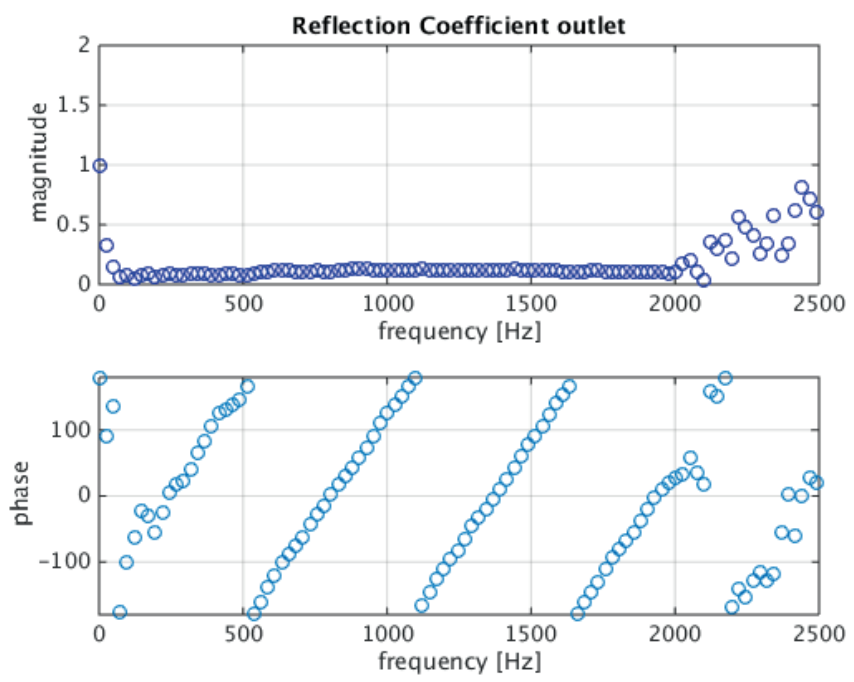

(b) $R_{2}$

Figure 3.5.: Reflection coefficient for divergent nozzle: no flow conditions 

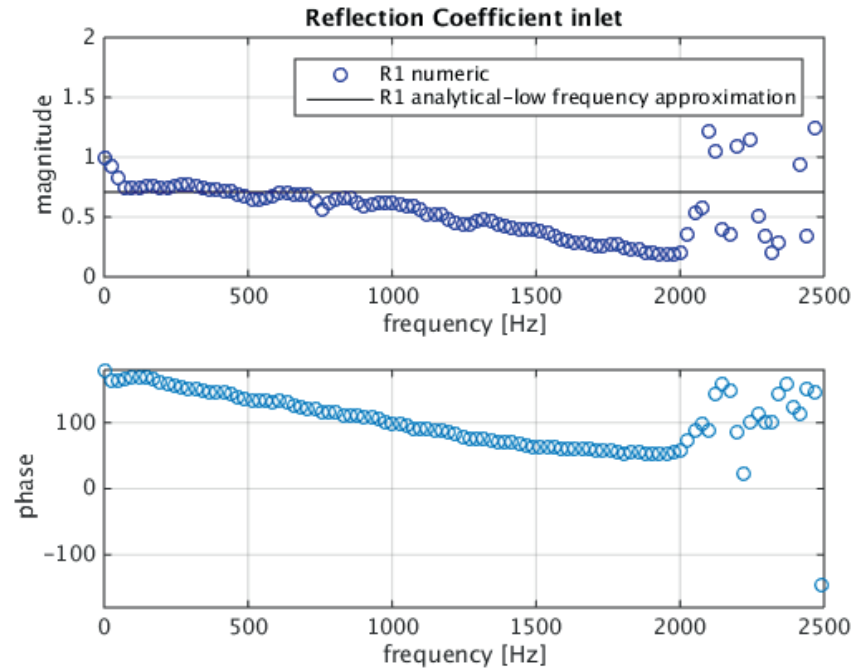

(a) $R_{1}-M_{\text {inlet }}=0.06, M_{\text {outlet }}=0.028$
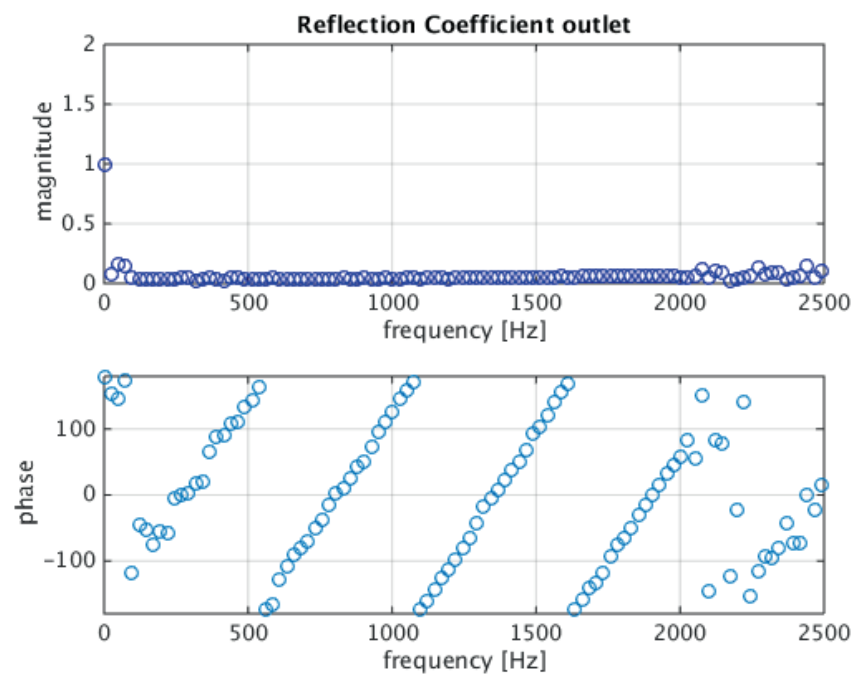

(b) $R_{2}$

Figure 3.6.: Reflection coefficient for divergent nozzle: flow conditions 

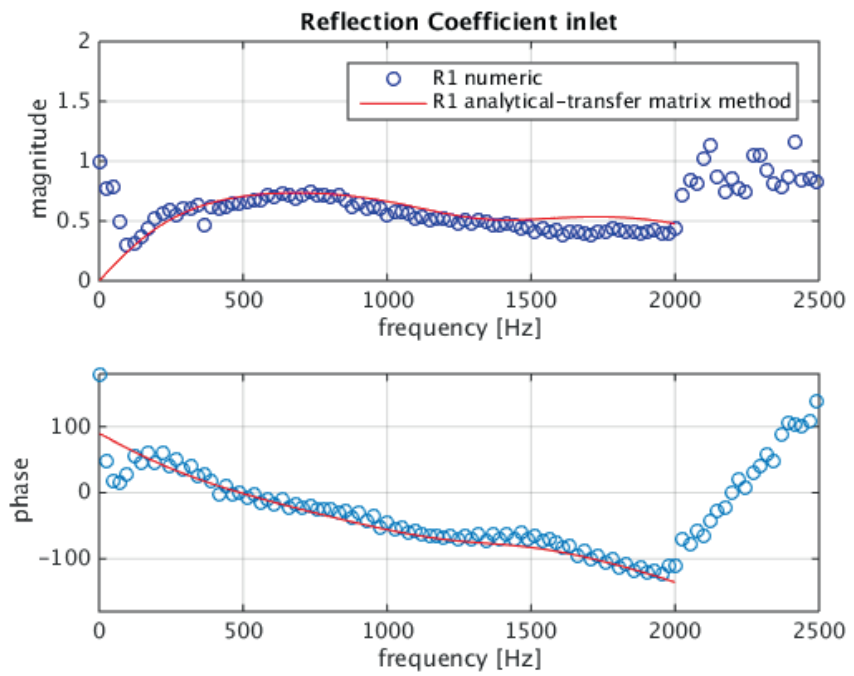

(a) $R_{1}$ - no flow
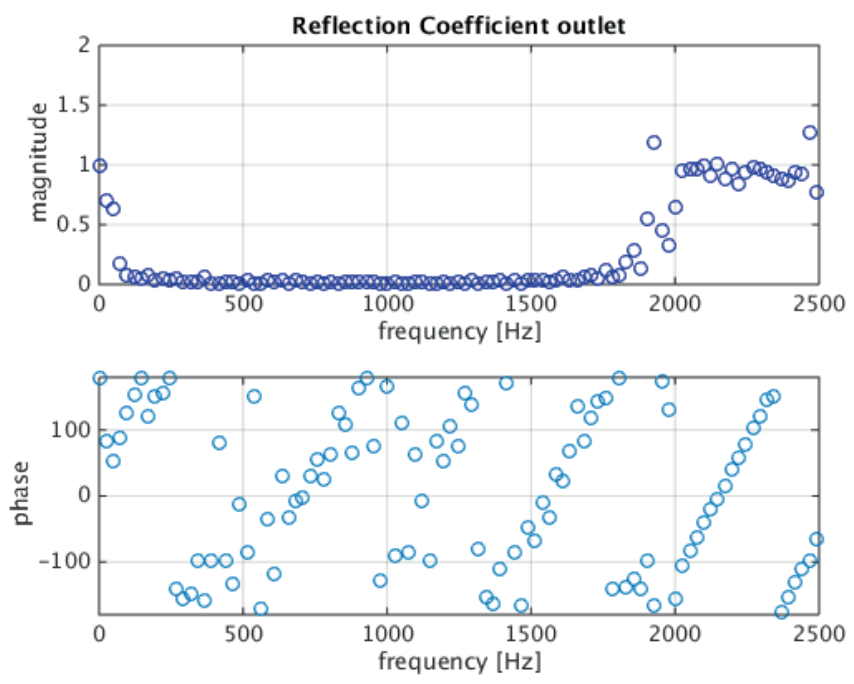

(b) $R_{2}$

Figure 3.7.: Reflection coefficient for convergent-divergent nozzle: no flow conditions 

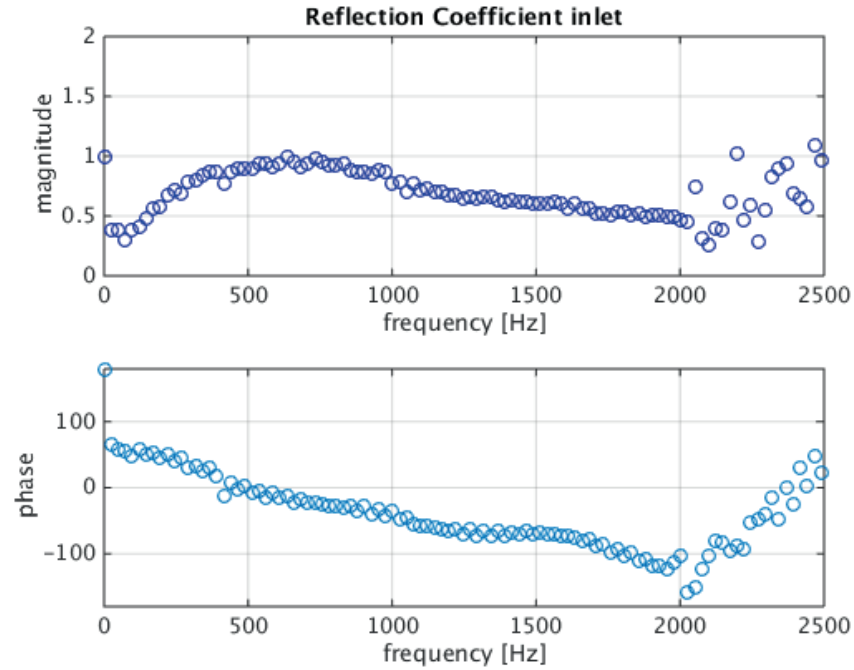

(a) $R_{1}-M_{\text {inlet }}=M_{\text {outlet }}=0.1, M_{\text {throat }}=0.49$
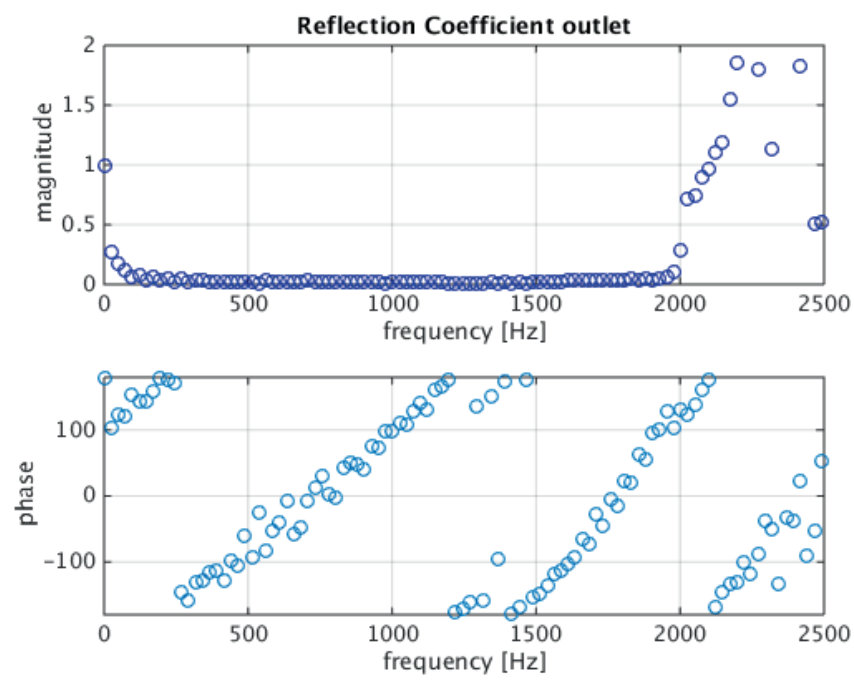

(b) $R_{2}$

Figure 3.8.: Reflection coefficient for convergent-divergent nozzle: flow conditions 
been obtained for the reflection at the inlet with the same trend in comparison with the no flow case but with higher values due to higher Mach number studied.

The same behavior is also presented for $R_{2}$ at the outlet in Fig. 3.8(b) verifying again the NRBC.

\subsection{4. $2 \mathrm{D}$ stator vane}

The case $2 \mathrm{dV}$ with vane has been studied in Fig. 3.9 and 3.10. This test case can be compared with a $3 \mathrm{D}$ real geometry when no gradient in the spanwise direction is present.

For reflection at the inlet witout flow in Fig. 3.9(a), the configuration has been compared also with the analytical transfer matrix method. Here the cross-sections have been approximated along the center stream line mapping the vane stage to a geometry similar to the CDN configuration. For the amplitude of $R_{1}$, the numerical curves follow the analytical formula with accurate agreement for about $f>400 \mathrm{~Hz}$. For low frequencies range the reflection is higher in the numerical results compared to the analytical method. The sudden change of the behavior at $f=400 \mathrm{~Hz}$ might be attributed to the quality of the CFD solution in the low frequency range, due to the choice of the relaxation factor in the settings of NRBC. For $f>400 \mathrm{~Hz} R_{1}$ is increasing reaching a flatter trend at $f=1500 \mathrm{~Hz}$ with $R_{1}=0.6$.

$R_{2}$ presents in Fig. 3.9(b) a smooth behavior around zero, but it shows also reflection for low frequencies.

In case of flow the curve of the magnitude of $R_{1}$ in Fig. 3.10(a) is steeper compared to no flow (Fig. 3.9(a)) with higher values of $R_{1}=0.8$ for the increased Mach number.

$R_{2}$ in situation of flow in Fig. 3.10(b) points out the same trend analyzed without flow underlying the correct behavior of the NRBC for $f>400 \mathrm{~Hz}$.

\subsubsection{Transfer matrix method extension to flow conditions}

As a following step, the analytical formulations obtained through the transfer matrix method need to be extended to the flow conditions with $0<M<1$.

In order to obtain this analytical solution, a 1D acoustic network tool by Siemens AG has been applied in order to compare the data with the numerical results with flow. A network model represents transport processes or more general relations forming a complex network which may even involve multiple feedback loops.

A model representing the geometries has been generated in Simulink, choosing various elements and nodes available in the solver. Each element can represent a boundary condition, a source of forced acoustical excitation or a duct section of variable area, and they are connected by nodes.

An example of a model used corresponding to the convergent-divergent nozzle CDN is shown in Fig. 3.11.

In this model, similar to the transfer matrix method, the profile is divided into various segments. Each segment represents a duct section of variable area and forced acoustic excitation can be applied upstream or downstream of these elements. The inlet and outlet boxes contain boundary conditions that are non-reflective and define the input mass flow, temperature and the output static pressure. 

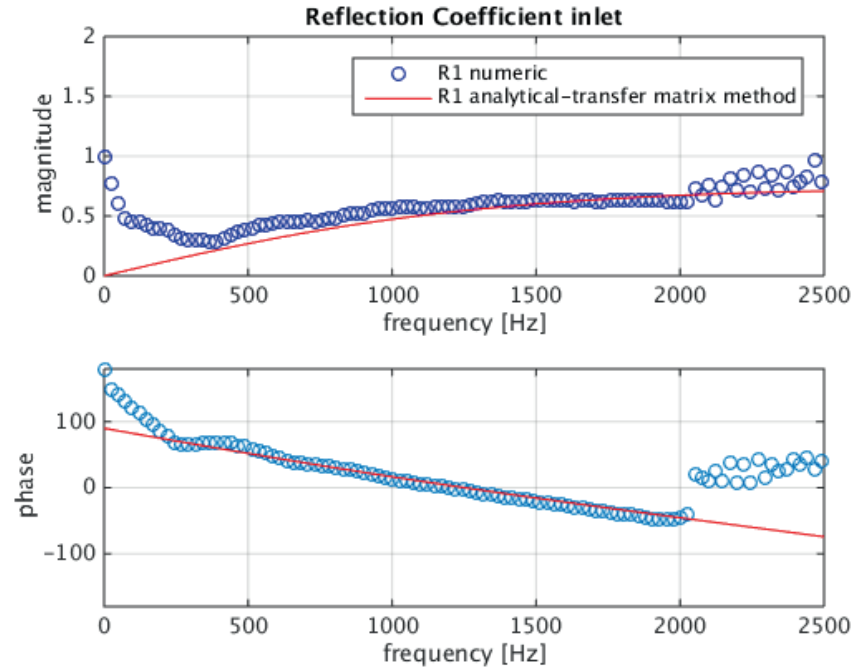

(a) $R_{1}$ - no flow
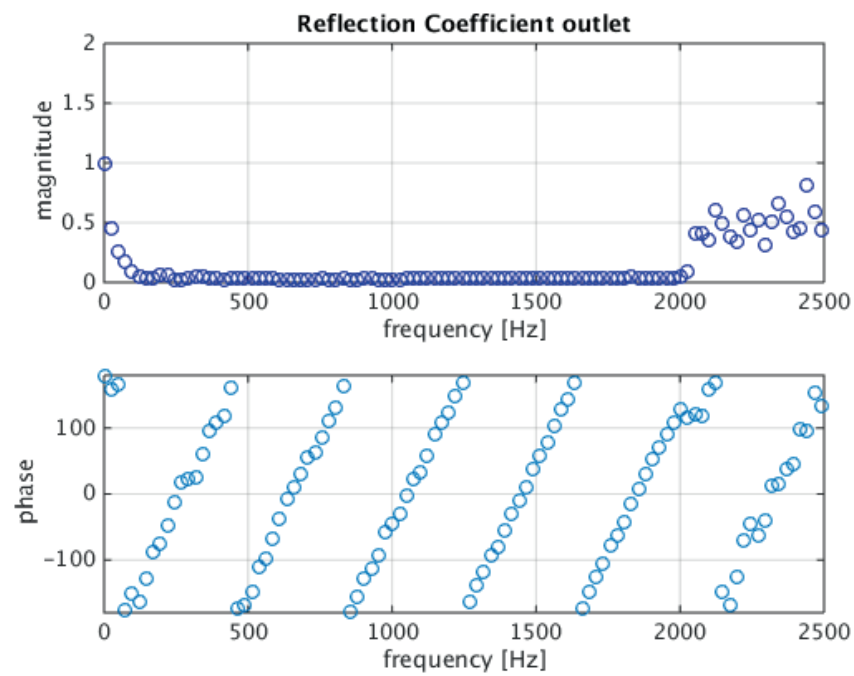

(b) $R_{2}$

Figure 3.9.: Reflection coefficient for $2 \mathrm{D}$ case with vane: no flow conditions 

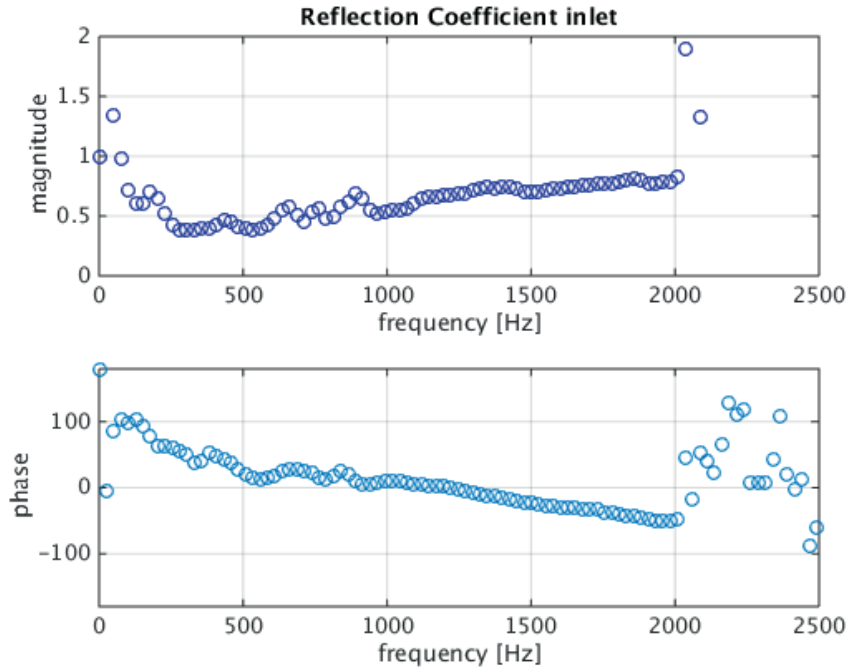

(a) $R_{1}-M_{\text {inlet }}=0.1, M_{\text {throat }}=0.25$
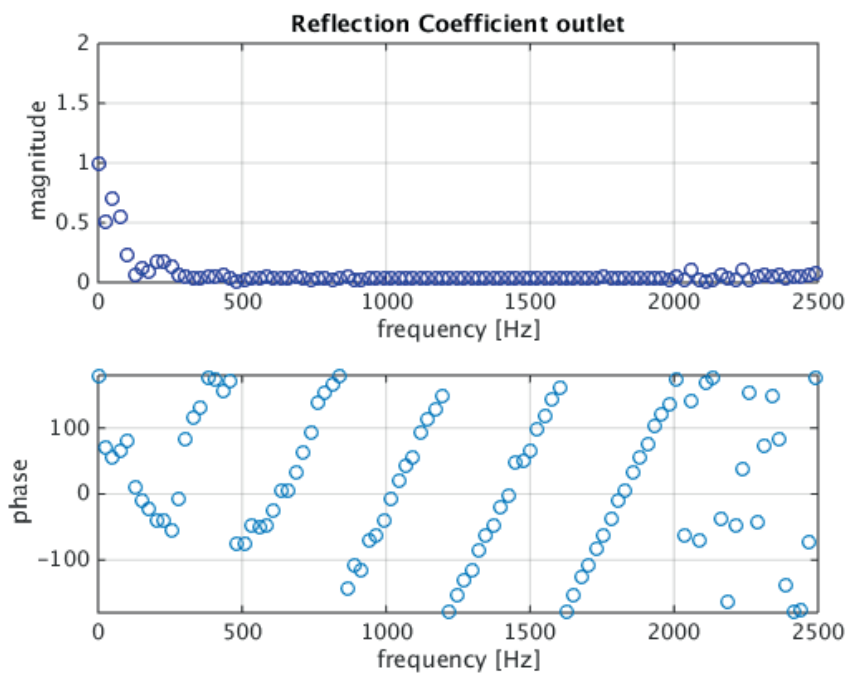

(b) $R_{2}$

Figure 3.10.: Reflection coefficient for $2 \mathrm{D}$ case with vane: flow conditions 


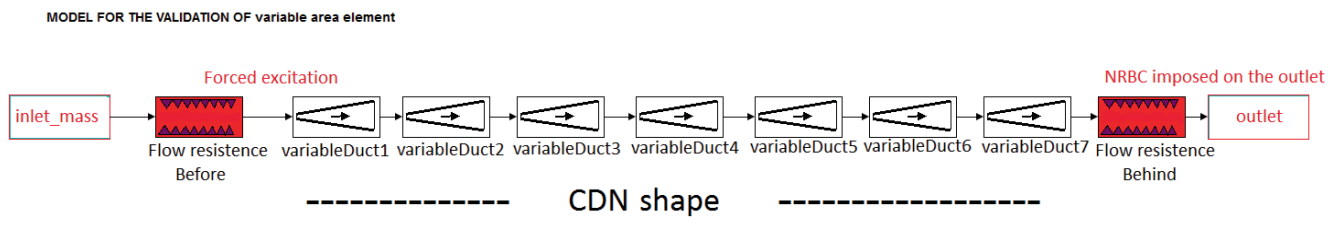

Figure 3.11.: Network model used for convergent-divergent nozzle CDN

In Fig. 3.12 the reflection coefficient magnitude behavior has been presented for the configurations CDN, CN and DN comparing the analytical formulations obtained with the network code with numerical simulations.

The convergent-divergent nozzle shows the most accurate agreement with the analytical formulae.

The other two geometries point out a similar trend with higher values of reflection around $R \approx 0.7$ for low frequencies. For frequencies $f>1000 \mathrm{~Hz}$, the numerics does not follow anymore the analytical results in a precise way. For $f>1000 \mathrm{~Hz}$ a $1 \mathrm{D}$ acoustic field is not necessarily the only solution, so CFD and 1D network model might diverge. As an improvement, simple diffuser losses could be added in the 1D model to take into consideration pressure losses occurring in the numerics. These modifications could improve the matching with the CFD results.

\subsection{Conclusion}

Geometries of different complexity have been investigated in this part and numerical results have been compared with analytical formulae.

The CFD code OpenFOAM has been used for the numerical analysis. A forced response approach has been studied provoking a wave excitation at the inlet of each geometry. For all $2 \mathrm{D}$ test cases a frequency range of $f=20-2000 \mathrm{~Hz}$ has been applied. A post-processing analysis through the two-microphones method has been performed.

The 2D test cases analyzed show agreement with the analytical frequency-dependent formulation in case of no flow.

The CN and DN present similar trends in case of $M_{\text {inlet }}>0$ reproducing the behavior of the analytical approximation of Ehrenfried for the low frequency range. The two nozzles show a decreasing behavior with higher frequencies $f>700 \mathrm{~Hz}$. The cases with flow present the highest values of $R_{1}$ compared with no flow tests. $R_{2}$ values at the outlet of each geometry confirm and verify the reliability of NRBC used.

As a next step, a convergent-divergent nozzle has been studied. For the case of no flow, the transfer matrix method has been considered also for this geometry that shows numerical accurate results compared to the analytical formula. In case of $M>0$, the numerical curve representing $R_{1}$ behavior, shows the same trend as in case of no flow. As expected the values of the reflection are higher due to the effects of the increased Mach number.

Calculations have been performed with another geometry of increased complexity: a $2 \mathrm{D}$ case with a vane deflecting the flow. The $2 \mathrm{dV}$ for no flow presents reliable agreement with the 
3. Thermoacoustic Influence of the Turbine on the Combustor - validation of tool chain

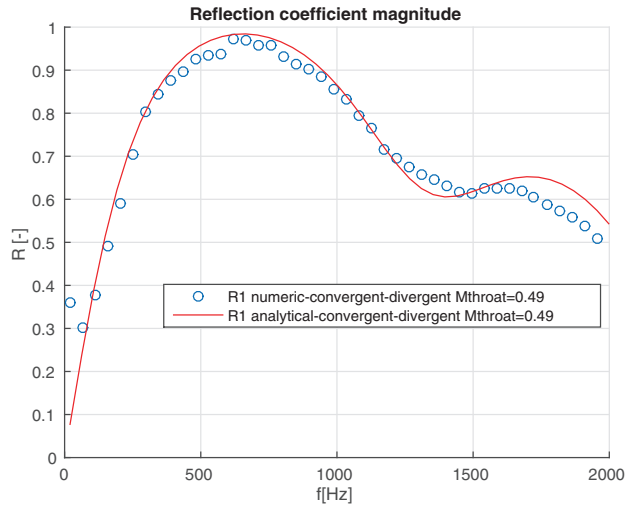

(a) Reflection coefficient magnitude for CDN

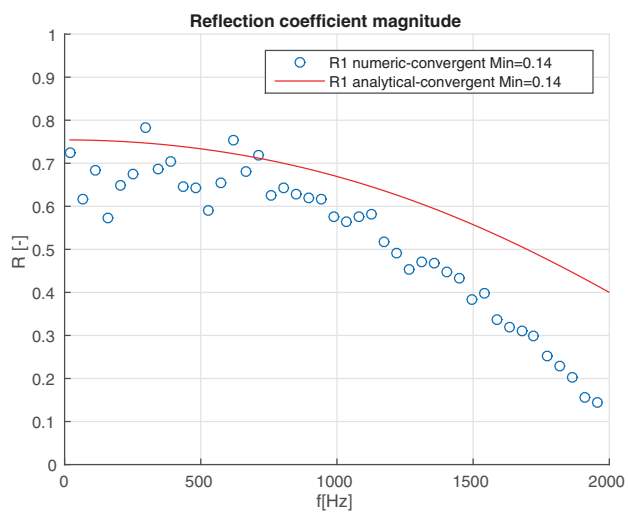

(b) Reflection coefficient magnitude for $\mathrm{CN}$

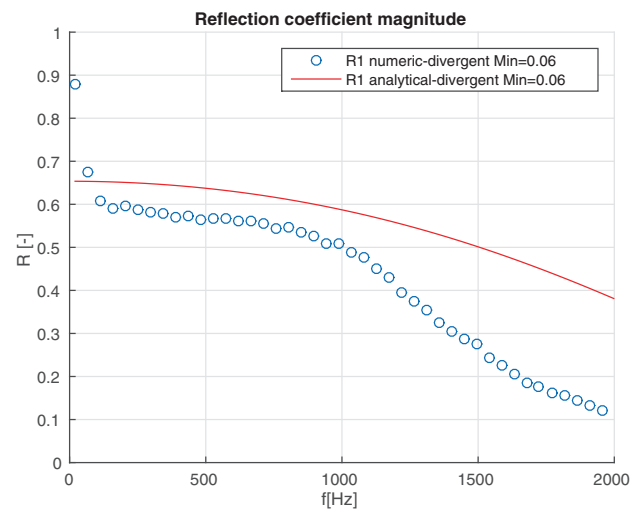

(c) Reflection coefficient magnitude comparisons for DN

Figure 3.12.: Reflection coefficient comparison between analytical formulation and numerical results obtained for CDN-CN-DN with flow 
analytical formulation obtained from the transfer matrix method. For the case with flow, the reflection at the inlet shows values of $R_{1}$ that are increasing for $f>500 \mathrm{~Hz}$.

An acoustic characterization method has been established proving the accurate ability of the numerical method to evaluate the acoustic propagation. 
3. Thermoacoustic Influence of the Turbine on the Combustor - validation of tool chain 


\section{Numerical Simulation of Sound Propagation through the Can-Annular Combustor Exit}

\subsection{Introduction}

The topic discussed below continues the previous analysis based on the reflection coefficient, extending the study also to 3D real configurations. The study is based on a simplified 2D geometry representing the vane section and a corresponding real engine alike combustor/turbine design. The real engine case consists of a can-annular combustion chamber sector and the first vane section. The investigation is based on numerical simulation methods allowing the analysis of complex geometries. The numerical results are compared to analytical formulae in order to test the validity of both approaches for the chosen geometries. 


\subsection{Investigated Configurations}

The cases selected for the analysis are shown in Fig. 4.1 and described below in more detail. The 2D stator vane passage (Fig. 4.1(a)) represents the configuration already described in section 3.2. This setup presents a good trade-off between accuracy and numerical effort. Due to periodicity, the passage has equal cross-sections before and after the profile. The 3D geometry (Fig. 4.1(b)) is a representation of a can-annular system often found in modern gas turbines. Since the 2D geometry is built with the same airfoil and equivalent cross-section, it is expected to obtain similar results for equal flow conditions. In terms of acoustic behavior it should be similar to the $2 \mathrm{D}$ single vane previously described.

\subsection{Numerical Method}

\subsubsection{LES Compressible Solver}

For all simulations compressible LES has been applied (more details concerning the numerical method used have been carried out in section 3.3.1). As discussed in section 2.4, a few LES models have been tested during the studies.

In parallel it was decided to run also simulations with laminar flow, since the main aspect of the work is related to the acoustic behavior of the systems. No turbulence was modeled applying a simplication of the flow. The results on sound propagation obtained with different approaches did not show any significant difference. The simulated physical time of the numerical calculations is up to $t=2 \mathrm{~s}$ for the $2 \mathrm{D}$ test case. For the $3 \mathrm{D}$ case a Courant number of $C o<1$ has been maintained with a typical time step $\Delta t=1 e-06 \mathrm{~s}$ running the simulation for about $200 \mathrm{~ms}$ real time.

\subsubsection{NRBC and Periodicity}

Non-Reflecting Boundary Conditions (NRBC) have been used at the inlet and outlet of the geometries. The role of the NRBC is to impose the flow conditions while suppressing acoustic wave reflections. The derivation of the NRBC is based on Local One-Dimensional Inviscid (LODI) as discussed in section 2.5. For the cases analyzed, the periodicity has been taken into account. Figure 4.1 shows the boundary conditions applied for both $2 \mathrm{D}$ and $3 \mathrm{D}$ configurations in detail. The periodic patches were conforming and no interpolation was necessary.

\subsubsection{Flow}

Each geometry has been investigated using different mass flows $\dot{m}$ at the inlet resulting in different Mach numbers. The operating conditions have been varied maintaining non-choked flow in the throat $(M<1)$ of the vane section. In the following only the case without flow and one with a Mach number in the throat $M \approx 0.7$ are presented. 


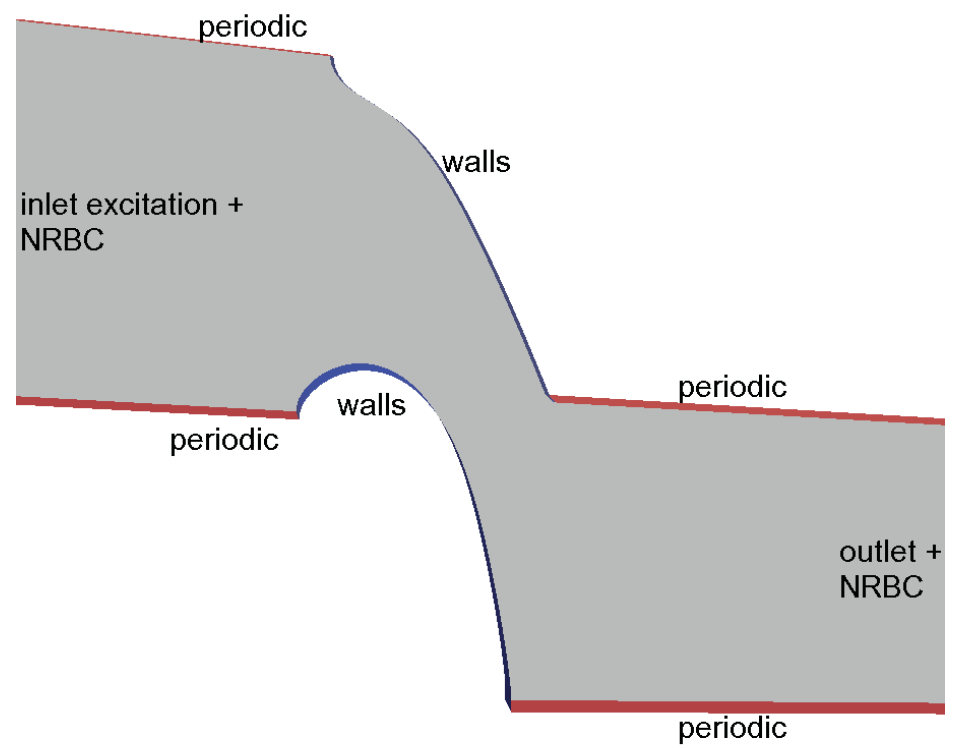

(a) $2 \mathrm{D}$ single vane geometry with periodicity

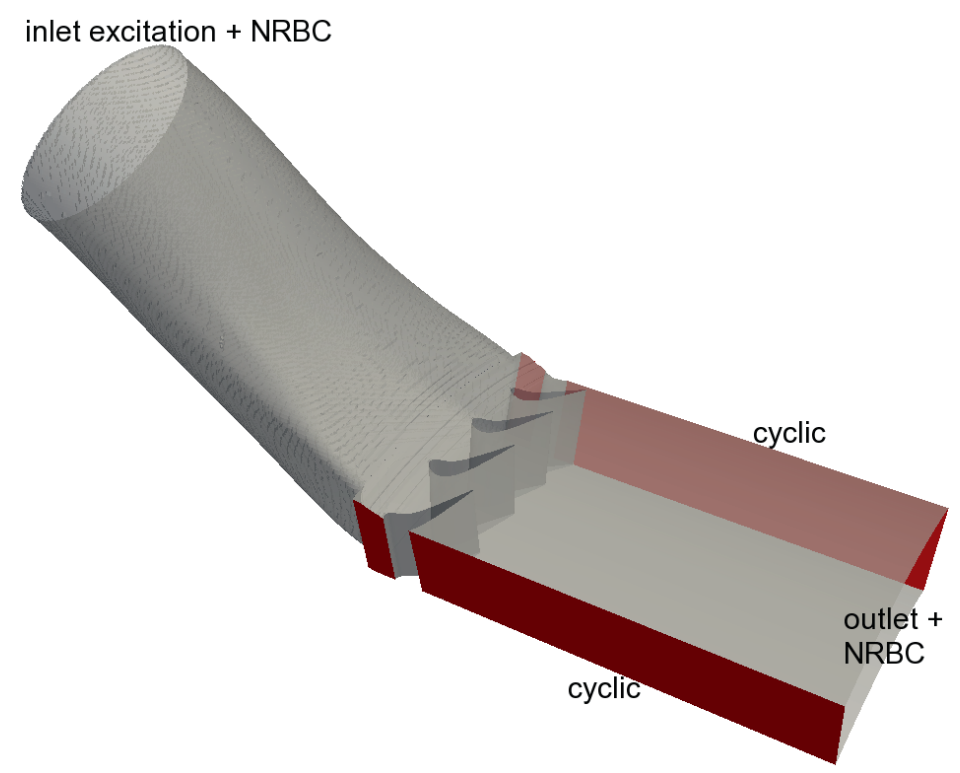

(b) 3D can and stator stage with artificial extensions upstream and downstream

Figure 4.1.: Overview of investigated geometries with definition of the boundary conditions applied 


\subsubsection{Meshing}

The computational mesh of the 2D case analyzed consists of about 70000 cells. The mesh has been generated through the tool BLOCKMESH with one cell in depth. Additional wall refinements have been used around the vanes in order to resolve the flow with more accuracy in this section. A cell stretch with a factor of 3 has been applied in the outlet area for an increased dissipation of flow structures hitting the outlet boundary. The mesh for the 3D engine case consists of about 3 million cells and the mesh has been also generated using the tool BLOCKMESH but additionally using the tool SNAPPYHEXMESH to mesh the complicated boundary surface.

\subsubsection{Forced Response Approach}

A forced response approach has been used during the simulations, imposing a sound excitation at the inlet of the combustion chamber. Multi-harmonic excitation with a wide frequency range of $f=20-2000 \mathrm{~Hz}$ has been used at small amplitudes of less than $1 \%$ of the mean values to stay in the linear regime. Pressure and velocity signals has been recorded at various points along the flow path during the numerical calculations. The post-processing for all cases is performed using the two and multi-microphone method in order to calculate the reflection coefficient taking into account the effects of the mean flow.

\subsubsection{Methods applied in the post-processing}

The two and multi-microphone methods have been applied for this analysis. The two methods have been described in detail in section 2.6.1 and section 2.6.3. For the 2D case and the 3D geometry, the leading edge of the vanes has been taken as reference. As already explained in section 2.6.1, the results quality obtained with the two-microphone method can decrease quickly for microphone distances $s>\lambda / 2$. This behavior can be observed in Fig. 4.2 and Fig. 4.3 where a comparison between the 2-microphone and the multi microphone method is presented. Figure 4.2 presents the amplitude of $R$ as a function of frequency. The amplitude is near 1 at very low frequencies, decreasing to approximately 0.6 above $f=300 \mathrm{~Hz}$. In contrast with the multi-microphone method, the two-microphone method shows erratic behavior, due to wave length sensitivity. In Fig. 4.3 the phase of $R$ can be observed as a function of frequency. The results obtained for the two-microphone method present a chaotic trend compared with the multi-microphone method. The case analyzed with the two-microphone method shows values oscillating around a virtual curve pointing out a small offset between the two methods applied. This outcome justified the usage of the multi-microphone method for more accurate evaluations of the results.

\subsubsection{Validity of planar wave assumption}

To better understand the results shown in the plots, a calculation of the cut-off frequencies has been performed for both geometries under study. Below this characteristic frequency no propagation of higher modes is possible and the sound field consists of planar waves. In this situation, the recorded amplitudes of the analyzed microphones do not depend on the 


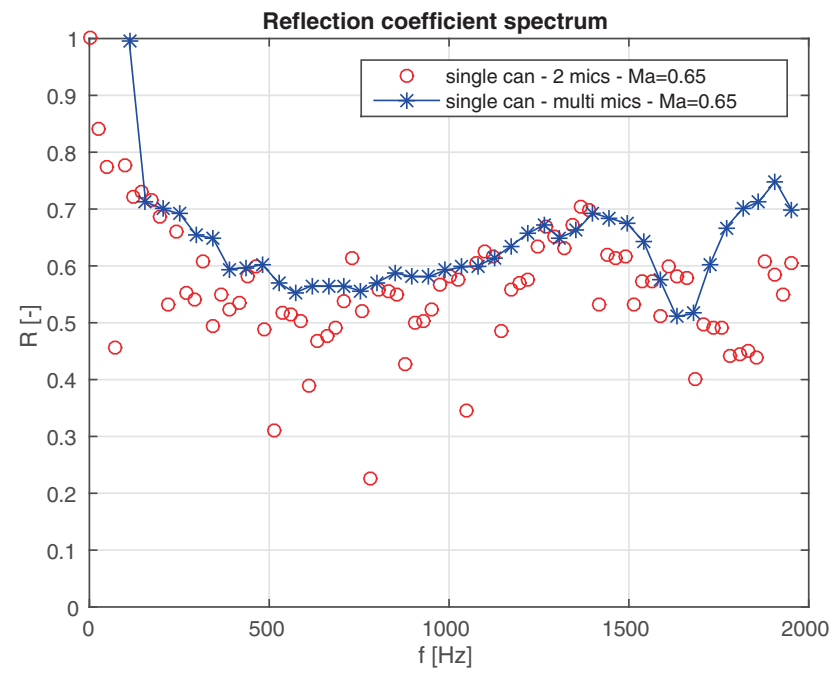

Figure 4.2.: Reflection coefficient magnitude for 3D configuration - multi-microphone method compared to two-microphone method

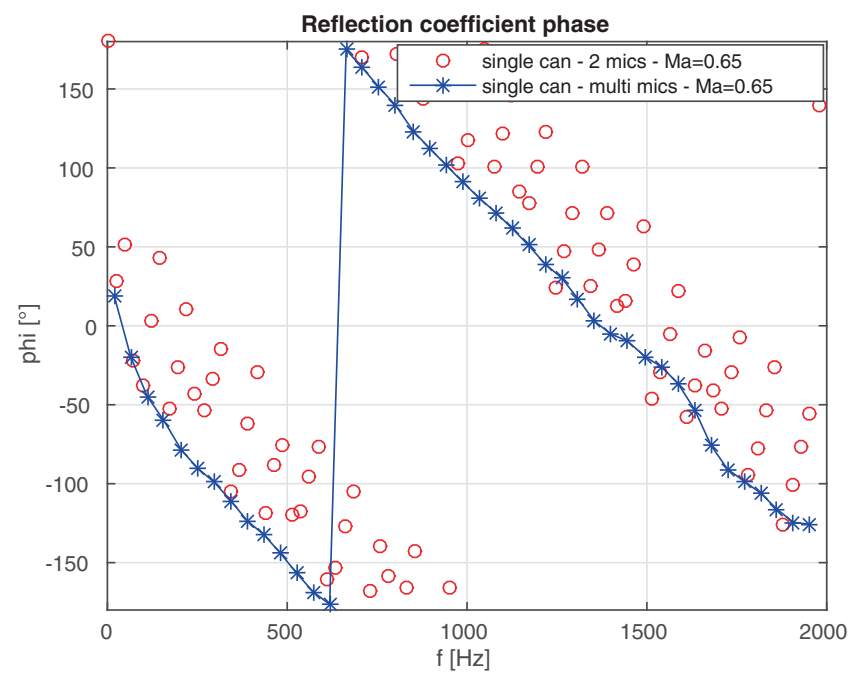

Figure 4.3.: Reflection coefficient phase for 3D configuration - multi-microphone method compared to two-microphone method 


\section{Numerical Simulation of Sound Propagation through the Can-Annular Combustor Exit}

transversal location in the duct. The cut-off limit is dependent on duct radius, mean Mach number and speed of sound. The values of the cut-off limits (see Eq. (2.24) in section 2.2.5) have been calculated based on the the cylindrical inlet section diameter for the 3D geometry and using the channel height $H$ for the $2 \mathrm{D}$ case.

For the 3D geometry the first higher radial and azimuthal mode can propagate above around $1300 \mathrm{~Hz}$, at least in the circular section where an analytical estimation can be applied. As a consequence, results above this frequency might reveal deviations due to transversal waves. For the $2 \mathrm{D}$ case there are no higher modes propagating within the excitation frequency range used.

\subsection{Analytical solutions}

To access the quality of the CFD results a comparison with analytical results is made where possible. Depending on the presence of flow, two types of analytical solutions are used for the comparison. For the no flow cases, the 1D transfer matrix method is used. This approach is often used in 1D network models to analyze longitudinal combustor instabilities. For the simulations with flow a formulation proposed by Cumpsty \& Marble [17] has been applied. In principle, both formulations could be applied with and without flow, but the passive deviation of the flow and acoustics propagation direction has to be considered first, for more detail see section 4.5.3.

In the case of a quiescent fluid, the thermodynamic state on both sides of the vane passage is equal. The sound reflection is only influenced by the area blockage. The question to answer here is at which angle the sound waves leave the passage. In case of an undisturbed propagation direction the blockage can be approximated with a straight convergent-divergent nozzle with equal inlet and outlet cross-section on either end. In this case the transfer matrix method is easily applicable. In case the sound waves follow the vane shape and deflect at least partially, the equivalent model for the transfer matrix would be a convergent nozzle, where the outlet cross-section is smaller than at the inlet.

For the case with a moving medium the flow acceleration and the corresponding drop in pressure and temperature has to be taken into account. Hence, for a correct reproduction of the thermodynamic conditions at the outlet the application of the 1D transfer matrix method is restricted to a convergent nozzle. To have a correct flow, the geometry needs to be convergent. It is not possible to have correct (accelerated) flow with a convergent-divergent geometry model. The straight convergent-divergent scenario allows equal flow conditions which are not realistic in a stator cascade.

In the following section, the formulation of Cumpsty and Marble is introduced. More details related to the transfer matrix method can be found in section 3.5.1.

\subsubsection{Formulation of Cumpsty and Marble}

An alternative analytical formulation to the transfer matrix method has been proposed by Cumpsty \& Marble [17] to calculate the propagation of sound and entropy waves in a stator vane cascade. This model is based on the principle of the compact nozzle of Marble \& Candel [19] but considers a two-dimensional configuration. The solution has been applied 
more recently by Leyko et al. [8] and compared to CFD results.

In this assumption (valid only in the limit of low frequencies) the characteristic wavelength $\lambda$ of waves is longer compared to the nozzle length $L, \lambda \gg L$. The paper of Leyko et al. [8] presents the formula of the reflection coefficient for a stator blade extended to flow without any references to the frequencies:

$$
R=\frac{w_{2}^{+}}{w_{1}^{+}}=\frac{\mu_{1}}{\mu_{2}} \frac{1+M_{1} \cos \vartheta_{1}+\frac{w_{1}^{-}}{w_{1}^{+}}}{1+\frac{M_{2}}{\cos \vartheta_{2}}}
$$

where $\mu=\left(1+(\gamma-1) M^{2} / 2\right)^{-1}, M_{1}$ and $M_{2}$ are respectively the Mach numbers at inlet and outlet of the geometry, $\vartheta_{1}$ and $\vartheta_{2}$ the flow angles at inlet and outlet, $w_{2}^{+}$reflected wave upstream and $\frac{w_{1}^{-}}{w_{1}^{+}}$with $w_{1}^{-}$reflected wave upstream and $w_{1}^{+}$incoming wave upstream side of the vane row:

$$
\frac{w_{1}^{-}}{w_{1}^{+}}=-\frac{1+\frac{1}{M_{1} \cos \vartheta_{1}}-\frac{\mu_{1}}{\mu_{2}} \frac{\left(1+M_{1} \cos \vartheta_{1}\right)\left(M_{2} \cos \vartheta_{2}+1\right)}{M_{2}\left(\cos \vartheta_{2}+M_{2}\right)}}{1-\frac{1}{M_{1} \cos \vartheta_{1}}-\frac{\mu_{1}}{\mu_{2}} \frac{\left(1-M_{1} \cos \vartheta_{1}\right)\left(M_{2} \cos \vartheta_{2}+1\right)}{M_{2}\left(\cos \vartheta_{2}+M_{2}\right)}} .
$$

This last formulation has been applied to the 2D single vane and 3D geometries (see Fig. 4.4) showing the same trend presented in Leyko et al.[8] in Fig. 4(b). The amplitude of $R$ is decreasing monotonically with the frequency from about 1.2 at $\vartheta_{2}=0$ to 0 at $\vartheta_{2}=1.5 \mathrm{rad}$.

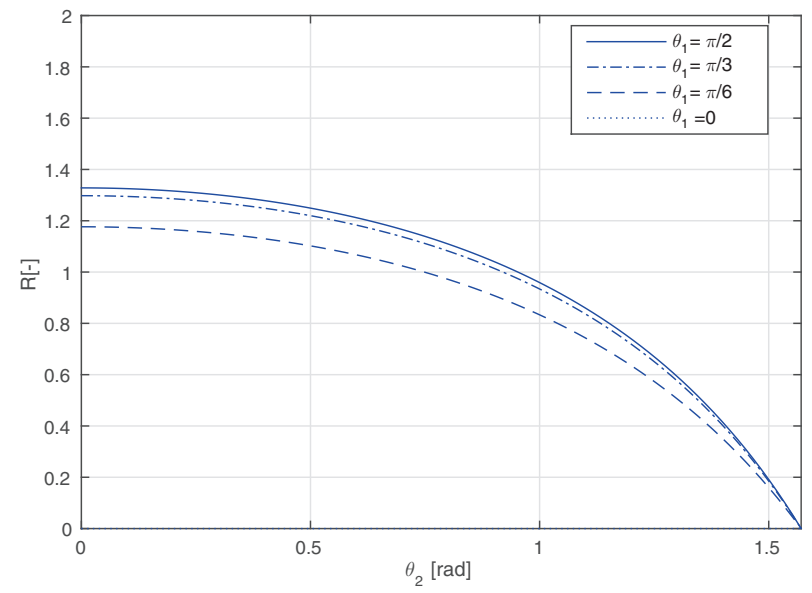

Figure 4.4.: Analytical formula in Eq.(4.1) applied to 2D single vane

\subsection{Results}

The numerical results obtained for the $2 \mathrm{D}$ and $3 \mathrm{D}$ configurations shown in the previous sections have been compared since their flow structures are equivalent. In this section a detailed study has been performed pointing out the acoustical behavior and comparisons for both geometries. 


\subsubsection{Flow Field}

At first, the flow obtained by the numerical flow simulation has been presented for both configurations. Figure 4.5 depicts the Mach behavior along the 2D and 3D geometries. For the $3 \mathrm{D}$ engine, the Mach trend has been shown along a cut plane containing the vanes. In the same plots also streamlines representing the deviation of the flow have been included. In Fig. 4.5(a) and Fig. 4.5(b) it can be observed how the flow is deflected by the vanes. Due to the deflection the flow is accelerated, leading to an increase of the Mach number to about 0.6. Figure 4.6 presents the plane wave impedance $Z_{0}=\varrho c$ obtained along the configurations. This quantity has been chosen to represent the thermodynamic state change caused by the acceleration of the flow in the stator. For the chosen Mach number, a decrease of $Z_{0}$ by a factor of 1.5 can be observed for both geometries.

\subsubsection{Reflection Coefficient}

The reflection coefficient obtained from the numerical simulations is analyzed for all configurations. The results analysed with the multi-microphone method have been compared and shown for the $2 \mathrm{D}$ and $3 \mathrm{D}$ configurations.

\section{Non-reflective termination}

The Non-Reflecting Boundary Conditions applied have been verified at the outlet. In the ideal case, the result is not influenced by unphysical waves reflected by the NRBC. The ideal reflection factor is hence $R=0$. In the investigated cases, the results obtained show an average reflection coefficient of 0.1 for intermediate and high frequencies. Little influence on the results can be expected. The factors grow to significant values for low frequecies and might result in inaccurate data in this range. The growth is attributed to the boundary condition formulation where a relaxation factor is used to set a trade-off between the non-reflectiveness and the controllability of mean values (see section 2.5 ).

$R_{\text {out }}$ represents the reflection coefficient measured at the outlet in order to validate the NRBC in both cases of no flow and flow shown in Fig. 4.7 and Fig. 4.8.

$R_{\text {out }}$ shows a smoother behavior around zero for the $2 \mathrm{D}$ configuration, but it shows reflection for low frequencies. In case of the 3D configuration the reflection at the outlet reached higher values around $R_{\text {out }}=0.1$ for the flow case, arriving to values around $R_{\text {out }}=0.2$ for the simulation without flow. For low frequencies all geometries present reflection that can be also in these cases attributed to quality of the CFD solutions.

\section{Reflection at the stator passage}

The results showing the reflection coefficient $R$ behavior have been calculated for the $2 \mathrm{D}$ and $3 \mathrm{D}$ geometries in the point located at the leading edge of the vane. The choice of the reference point has no influence on the reflection coefficient magnitude, but influences the phase. 


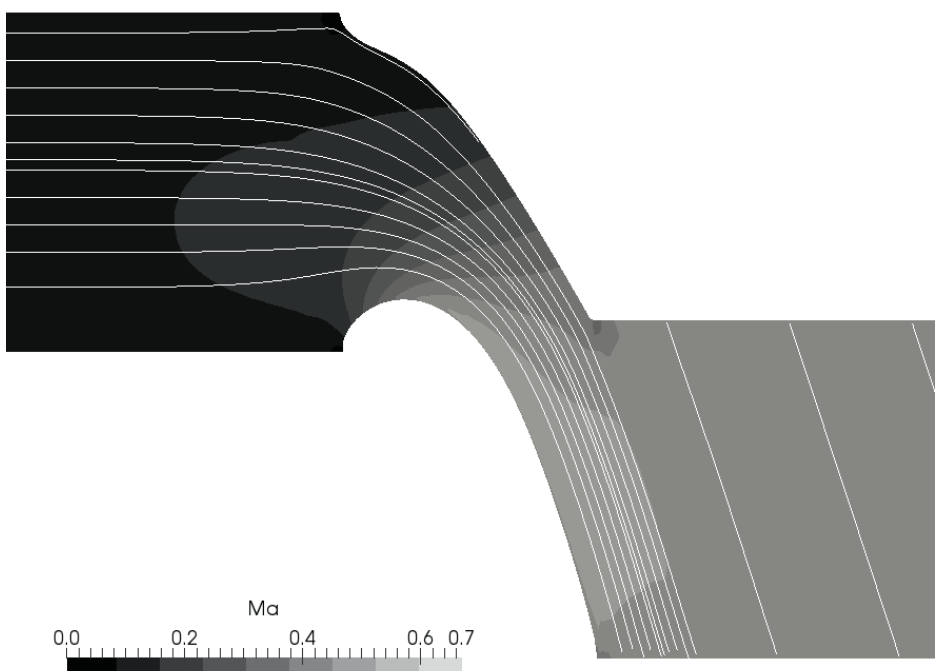

(a) $2 \mathrm{D}$ single vane
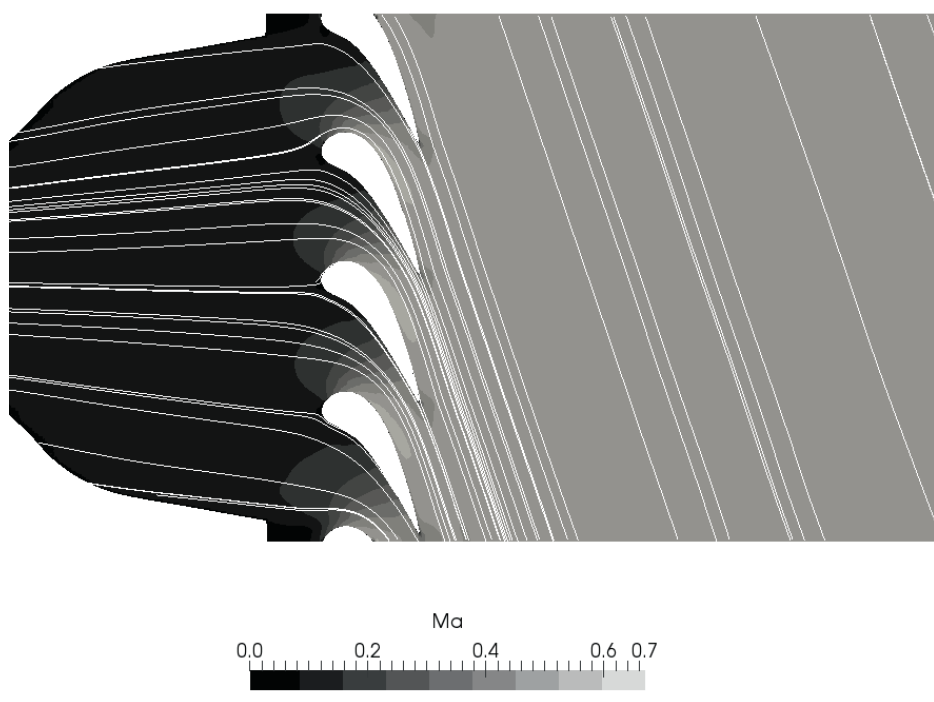

(b) Slice cut of the 3D geometry

Figure 4.5.: Distribution of the Mach number in the stator passage 
4. Numerical Simulation of Sound Propagation through the Can-Annular Combustor Exit

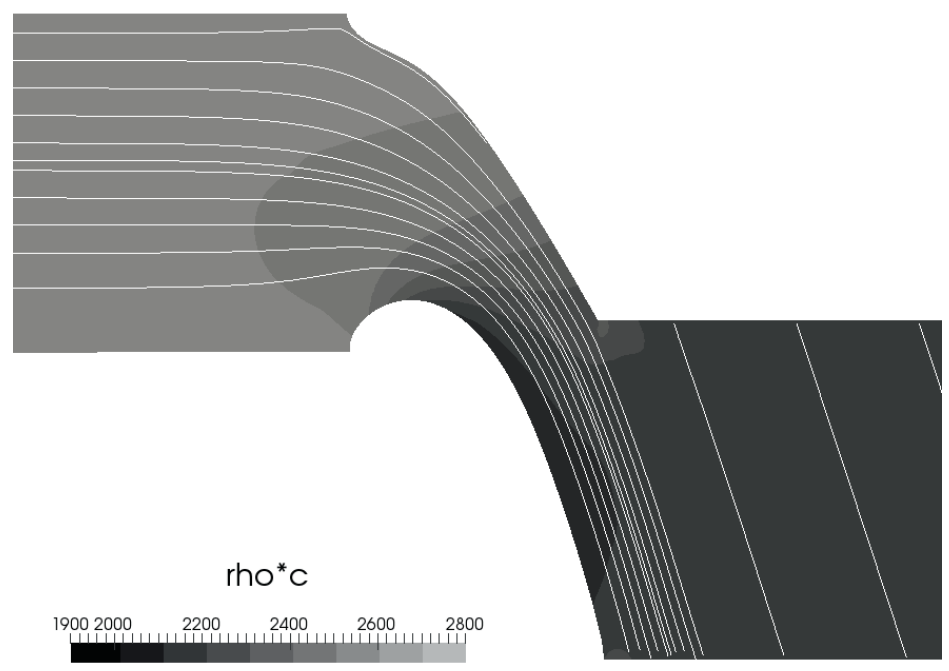

(a) 2D single vane case

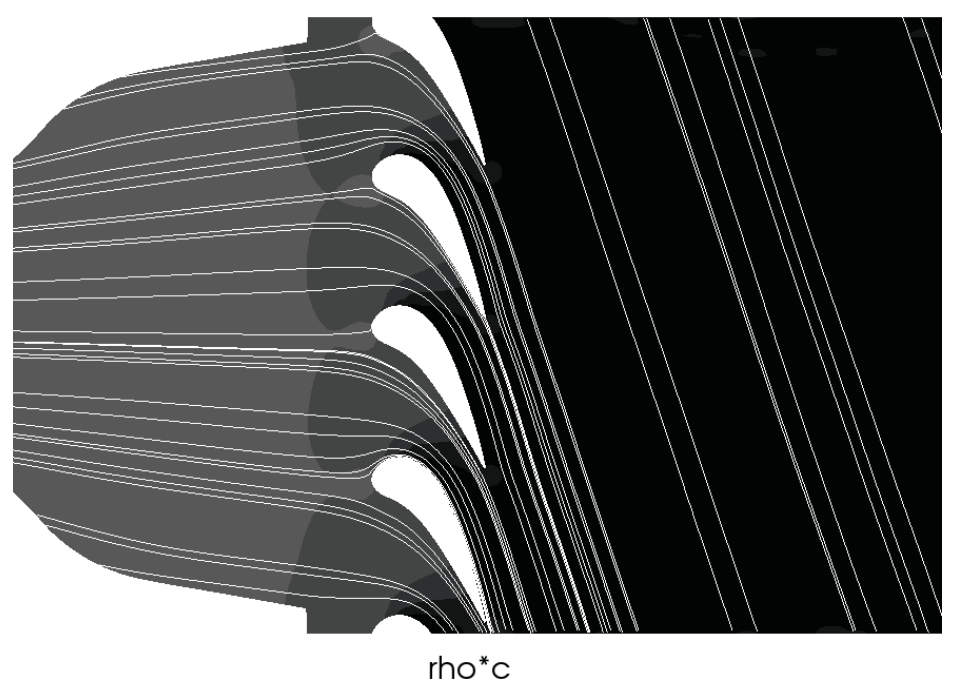

$19002000 \quad 2200 \quad 2400 \quad 2600 \quad 2800$

(b) Slice cut of the 3D geometry

Figure 4.6.: Distribution of the wave impedance in the stator passage 


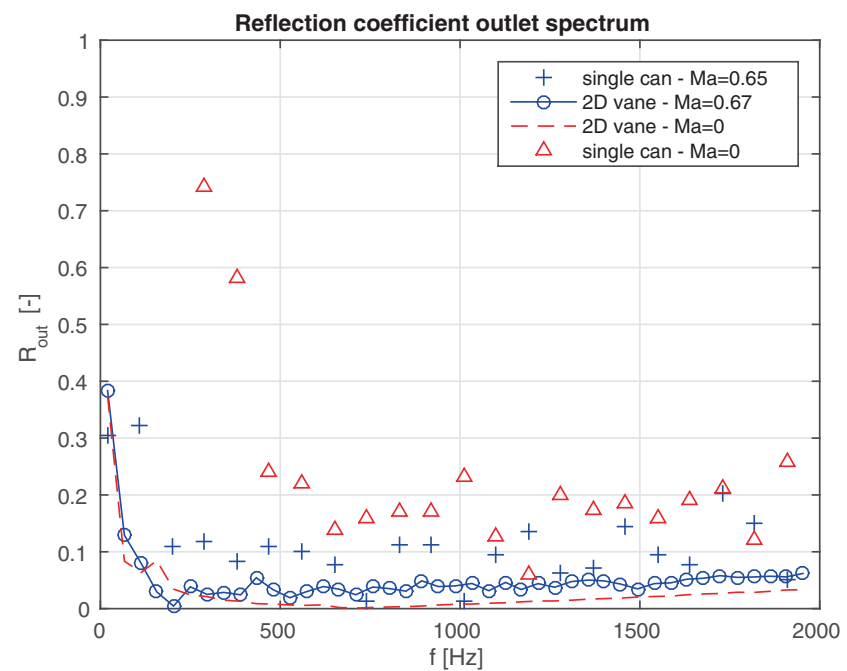

Figure 4.7.: 2D versus 3D configurations - Reflection coefficient spectrum at outlet

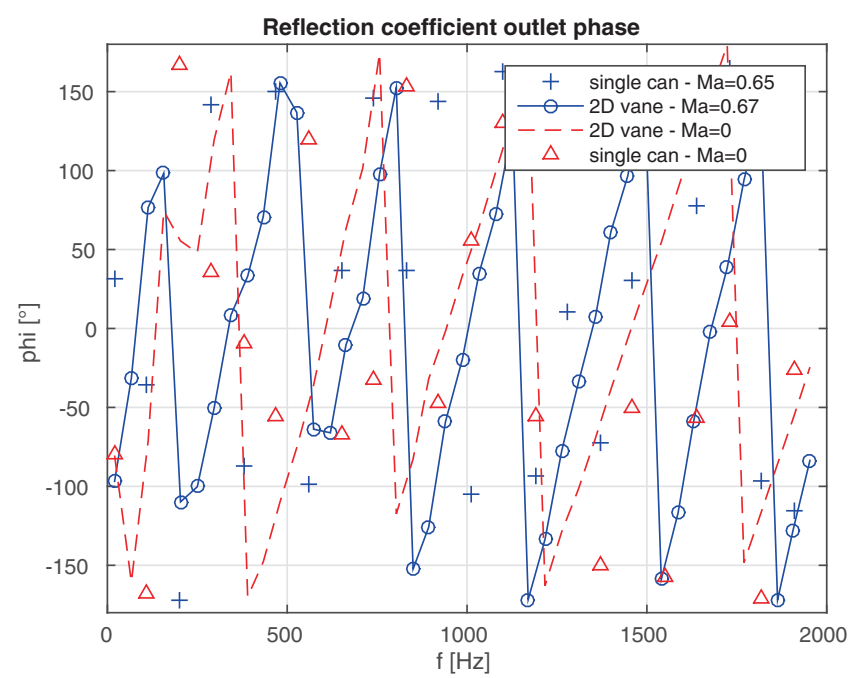

Figure 4.8.: 2D versus 3D configurations - Reflection coefficient phase spectrum at outlet 


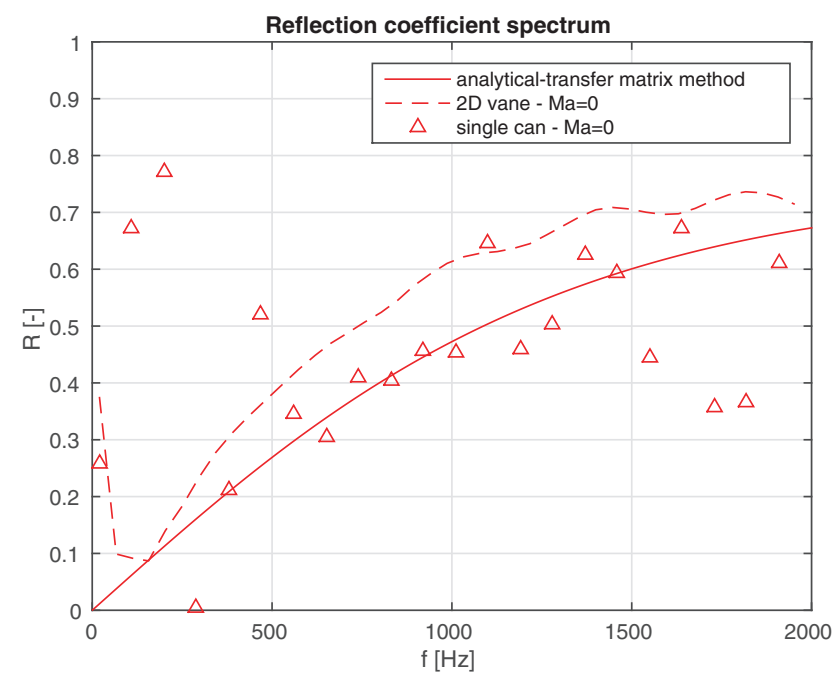

Figure 4.9.: Reflection coefficient magnitude for 2D and 3D cases compared to analytical formulation in Eq.(3.3): no flow

\section{Reflection without flow}

The 2D vane has been studied in Fig. 4.9 and Fig. 4.10 compared with the 3D geometry and with the analytical formulation in Eq. (3.3).

For these no flow cases, the numerical simulations have been compared with the analytical transfer matrix method. The vane passage cross-sections have been approximated along the center stream line mapping the vane stage to a $1 \mathrm{D}$ geometry similar to a convergent-divergent nozzle configuration. For $R$ analyzed before the vane section, the numerical curve representing the $2 \mathrm{D}$ geometry follows the analytical formula presenting the same trend. The reflection is higher in the $2 \mathrm{D}$ numerical results compared to the analytical method. The behavior shown by $R$ reaches a maximum value around $R=0.7$ maintaining then a flatter trend starting from $f=1500 \mathrm{~Hz}$. The numerical results for the 3D engine shows good agreement with the analytical formulation beyond $f=500 \mathrm{~Hz}$. The change of the behavior at $f=500 \mathrm{~Hz}$ might be attributed to quality of the CFD solutions in the low frequency range.

\section{Reflection with flow}

For situations with flow (see Fig. 4.11 and Fig. 4.12), the numerical results for all configurations have been compared with the low frequency approximation by Leyko et al. [8] in Eq. (4.1). Since both configurations present the same flow angle and Mach conditions at inlet and outlet, the same results obtained from the analytical formulation have been taken into account as comparison with the numerical simulations. In case of flow the curves representing the numerics are steeper with higher values of $R$ between $R=0.6$ and $R=0.8$ for the in- 


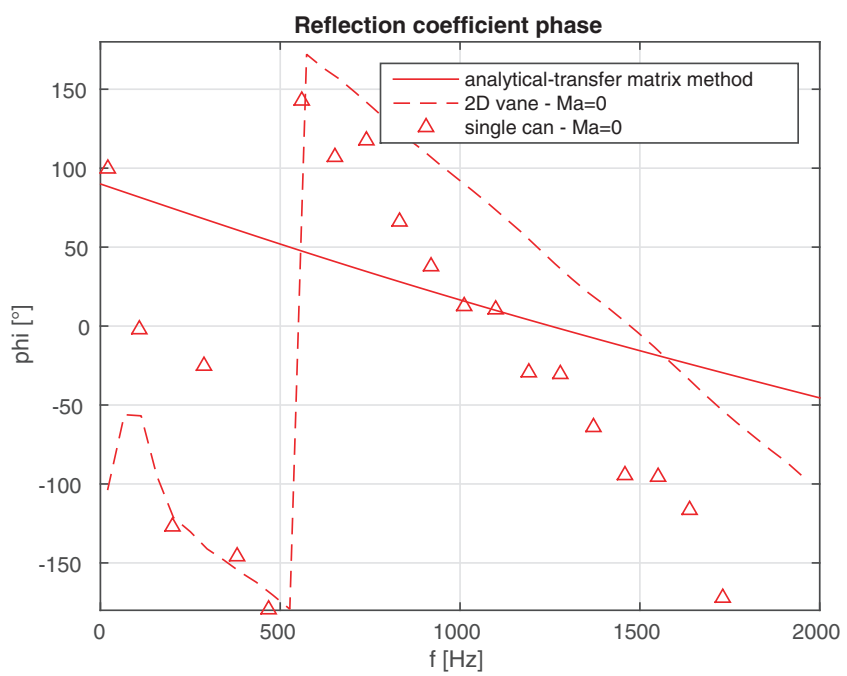

Figure 4.10.: Reflection coefficient phase for $2 \mathrm{D}$ and $3 \mathrm{D}$ cases compared to analytical formulation in Eq.(3.3): no flow

creased Mach number. The analytical formula does not consider the effects of the turbulence contrary to the numerics. This might in part explain the overall higher values of reflection coefficient shown in the numerics compared to the analytical approximation. Figure 4.13 and Fig. 4.14 show the flow study performed for the 2D single vane.

Several simulations have been run starting with a case without flow arriving to Mach number $M \approx 0.8$. As shown before for no flow, the reflection curve shows a trend with values that are increasing from $R=0$ arriving to $R=0.7$. In case of flow with $M=0.67$ the curve is steeper maintaining constant at higher values of $R$ between $R=0.6$ and $R=0.8$. For higher Mach number $M=0.81$ the curve shows much higher values of reflection coefficient as expected with constant trend at $R=0.75$. Figure 4.15 and Fig. 4.16 present the comparisons between the 2D geometry and 3D real engine for $M=0.67$ and $M=0$.

The two curves with high Mach show an accurate agreement underlying a similar trend of reflection coefficient with overall constant values around $R=0.7$. For no flow, the behavior of the 3D geometry follows the trend of the other curve for $f>500 \mathrm{~Hz}$ but it shows higher values of reflection for low frequencies up to $f=500 \mathrm{~Hz}$.

\subsubsection{Analysis of the sound propagation direction}

An interesting aspect of the sound transmission though an airfoil passage is the change of the sound propagation angle. While the deflection of the mean flow is obvious, the behavior of the acoustic perturbations behind the passage is not. Based on the detailed numerical data available, an analyis of the radiation direction has been performed.

The procedure is described below. For reason of geometrical simplicity the 2D case has 
4. Numerical Simulation of Sound Propagation through the Can-Annular Combustor Exit

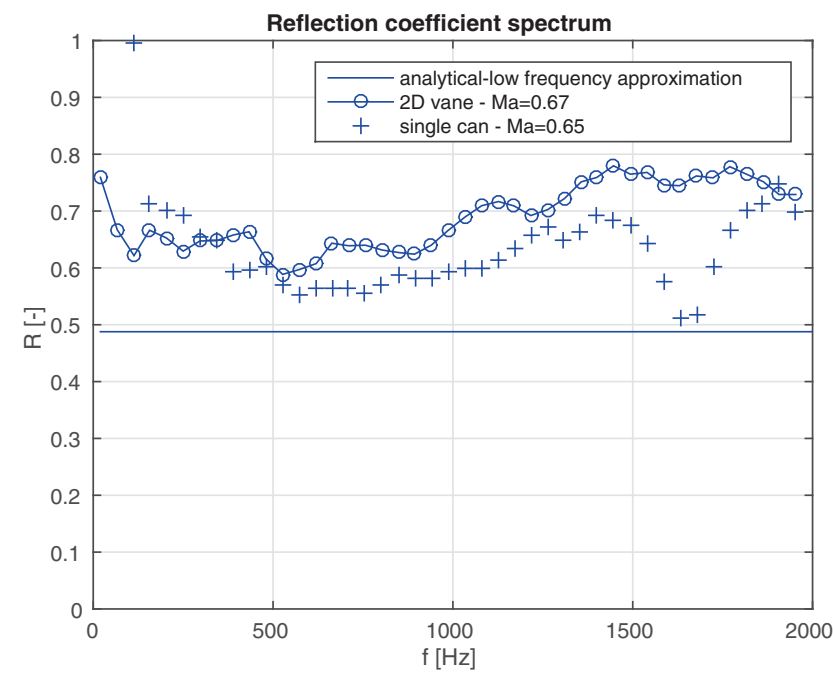

Figure 4.11.: Reflection coefficient magnitude for $2 \mathrm{D}$ and $3 \mathrm{D}$ cases compared to analytical formulation in Leyko et al.[8] in Eq.(4.1): flow

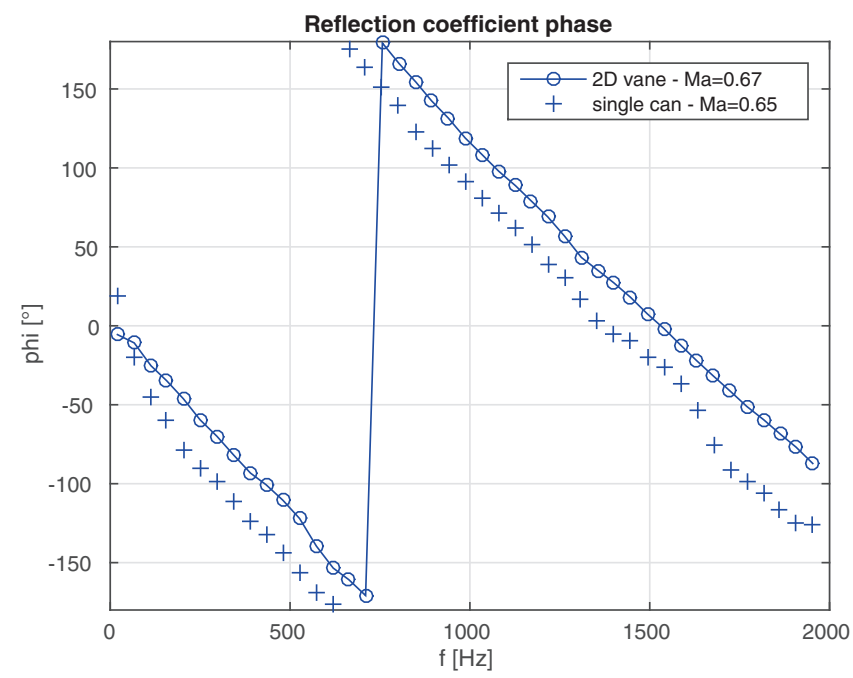

Figure 4.12.: Reflection coefficient phase for $2 \mathrm{D}$ and $3 \mathrm{D}$ cases compared to analytical formulation in Leyko et al.[8] in Eq.(4.1): flow 


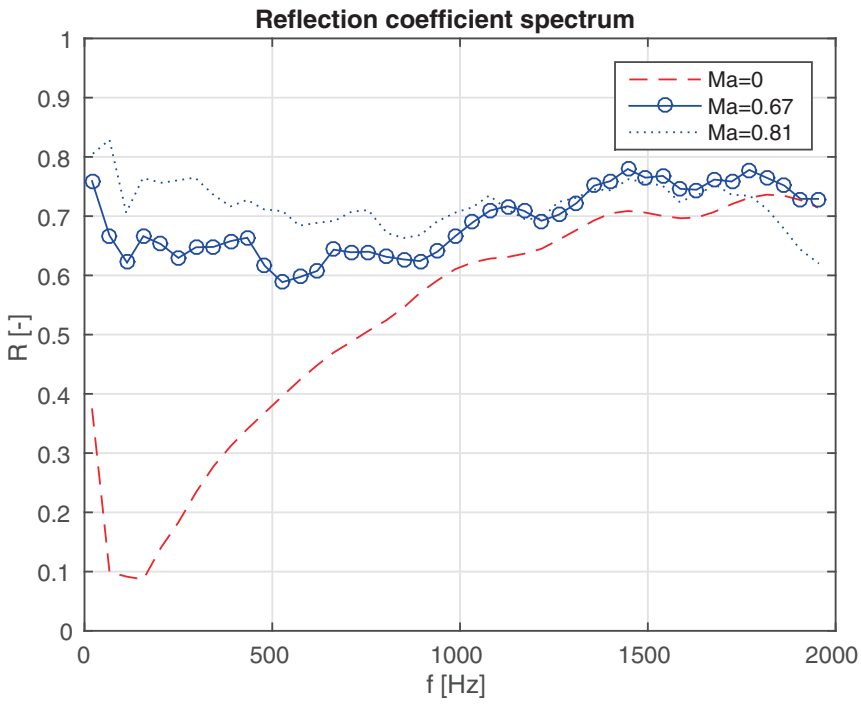

Figure 4.13.: 2D single vane flow study - Reflection coefficient magnitude

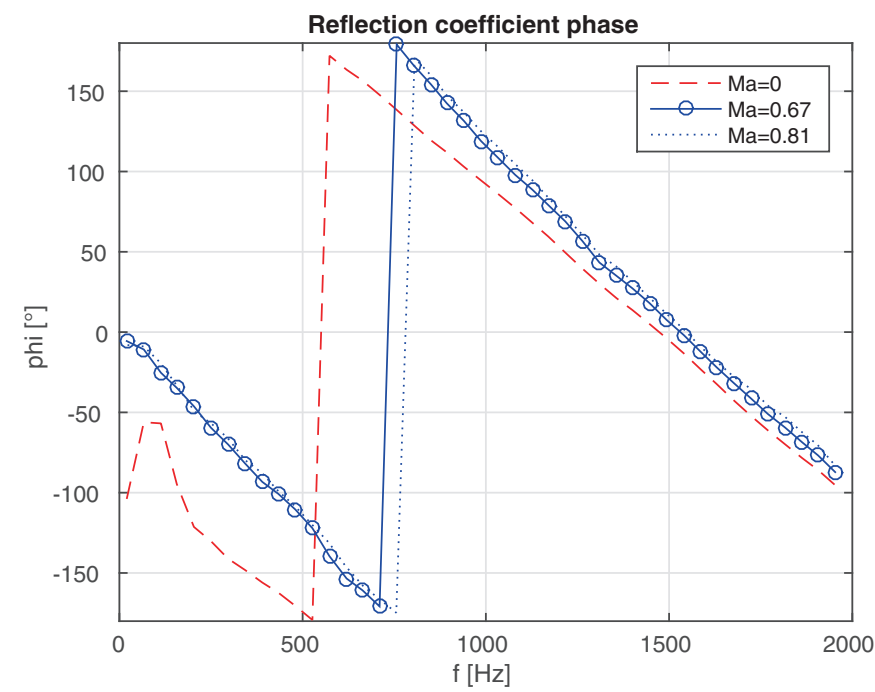

Figure 4.14.: 2D single vane flow study - Reflection coefficient phase 
4. Numerical Simulation of Sound Propagation through the Can-Annular Combustor Exit

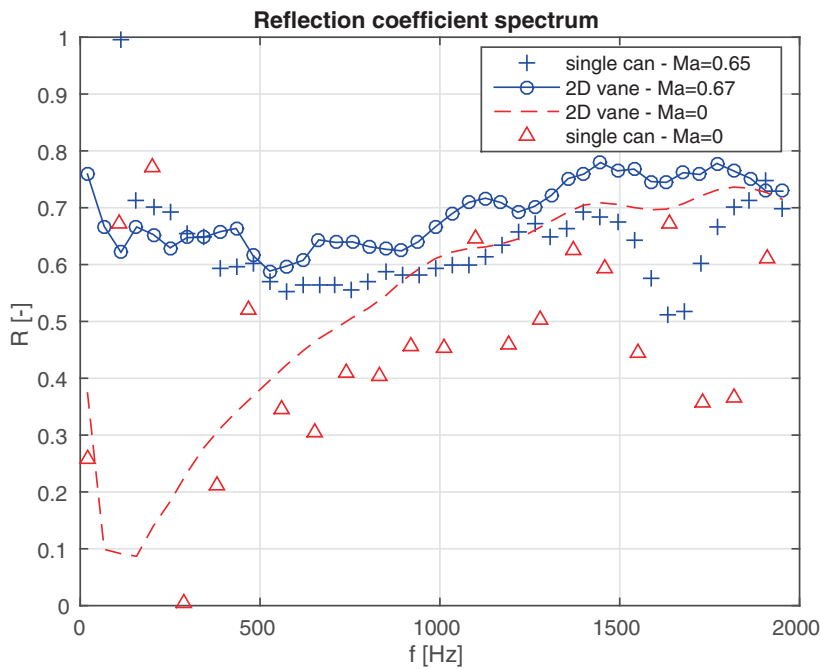

Figure 4.15.: 2D versus 3D configurations - Reflection coefficient magnitude

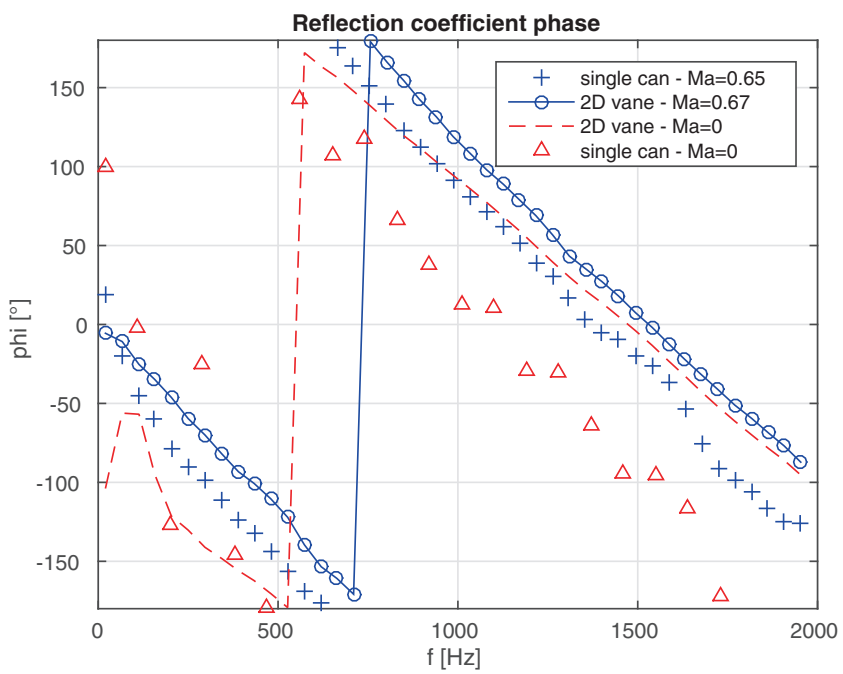

Figure 4.16.: 2D versus 3D configurations - Reflection coefficient phase 


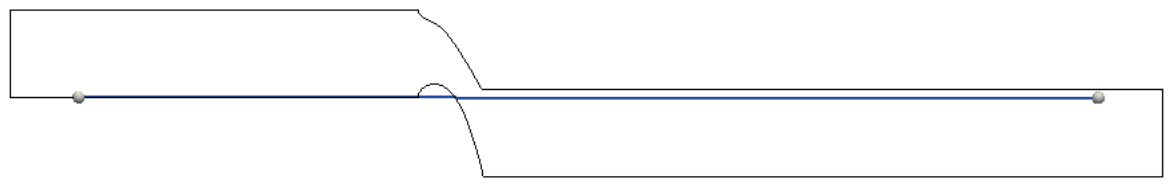

(a) 2D vane - axial distance taken into account for the analysis

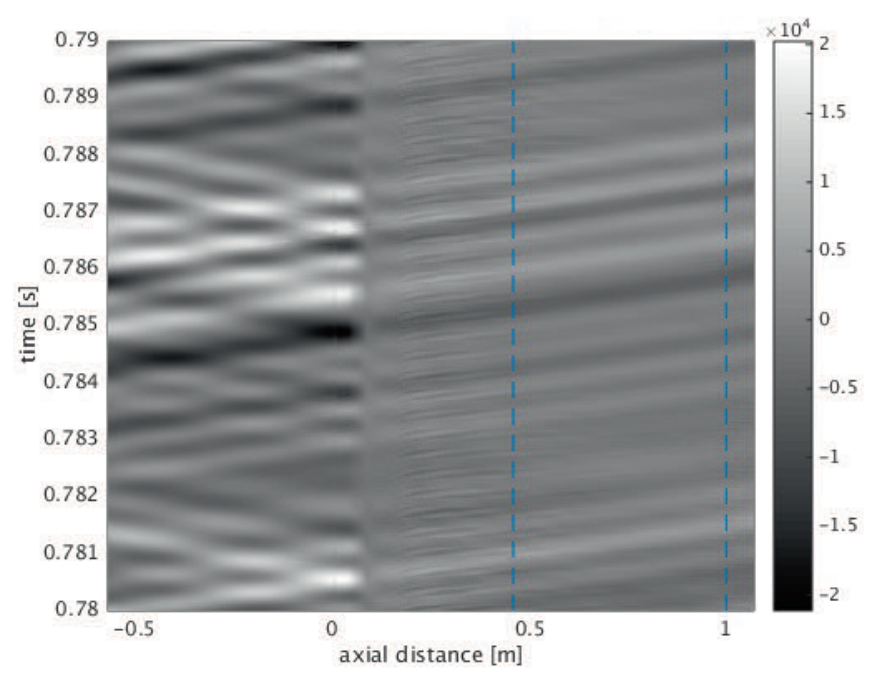

(b) 2D vane - time evolution of the signal analyzed

Figure 4.17.: 2D vane sound propagation angle study

been used for the investigation. The unsteady pressure signal has been sampled along a line spanning from the inlet section to the outlet of the computational domain. The line location and the data plotted over space and time are depicted in Fig. 4.17(a). The plot reveals characteristic lines of constant positive and negative inclination representing downstream and upstream propagation of sound. Some steeper lines attached to the vane indicate convective propagation of turbulent structures over a short distance.

To gain insight into the sound propagation angle behind the vane, the propagation delay between two points on the lines (indicated by the two dashed lines in Fig. 4.17 (b) at $x=0.45 \mathrm{~m}$ and $x=1 \mathrm{~m}$ ) has been calculated using the cross-correlation of the temporal signals at these two locations. The available data from the CFD results, namely the flow velocity components, speed of sound (from temperature) and the calculated propagation delay can be combined graphically as shown in Fig. 4.18. The average convective speed vector $\left[U_{x}, U_{y}\right]$ is the origin of the circle with a radius equal to the average speed of sound $c$ in the section downstream of the vane. The effective axial propagation velocity is derived from the cross-correlation delay and the line distance. It is indicated by the vertical dashed line in the plot at about $1000 \mathrm{~m} / \mathrm{s}$. Knowing that sound propagates axially with $U_{x}+c$, the propagation angle can be found by 


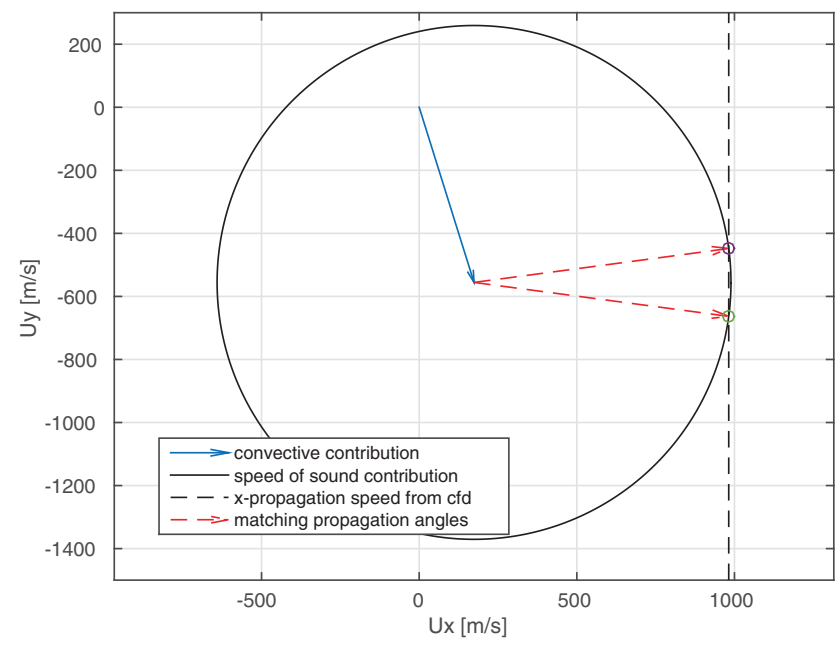

Figure 4.18.: 2D vane sound propagation angle analysis

finding the intersection of the vertical line and the circle. The intersection in the particular case is two-fold and at about $\pm 7^{\circ}$. Both solutions are mathematically valid, but the solution closer to the flow direction appears more physical. In conclusion, the acoustic propagation angle changes only slightly when the sound passes the stator stage.

\subsection{Conclusion}

Two models of different complexity have been analyzed in this chapter and numerical results have been compared with analytical formulae. A detailed study of the reflection coefficient $R$ behavior before the vanes has been performed for the $2 \mathrm{D}$ and $3 \mathrm{D}$ geometries. The $2 \mathrm{D}$ geometry and the 3D real engine present equivalent flow structures giving the possibility to make an accurate comparison. In case of no flow the configurations have been compared with the analytical formulation of 1D transfer matrix method. For comparison with the numerical results, a convergent-divergent nozzle has been chosen as equivalent model for the 1D transfer matrix. This approximation has been proved in this work pointing out that the sound wave propagation follows quite straight the vane shape. The numerical results follow the analytical trend overall in an accurate way. In case of flow the behavior of the analytical approximation shown in Leyko et al. [8] has been valuated and compared with the 2D and 3D numerical results. It needs to be taken into account that the analytical formula does not consider the effects of the turbulence contrary to the numerics. This can explain the overall higher values of reflection coefficent shown in the numerics compared to the analytical approximation. The numerical results follow the analytical trend with reasonable agreement. The sound propagation angle behind the vane has been also analyzed. For higher Mach 
number of about 0.7 a small deviation of about $7^{\circ}$ from the axial direction has been revealed. The numerical results shown for both $2 \mathrm{D}$ and $3 \mathrm{D}$ geometries pointed out overall a good agreement between the two configurations as expected. 
4. Numerical Simulation of Sound Propagation through the Can-Annular Combustor Exit 


\section{Thermo-acoustic Coupling in Can-Annular Combustors}

\subsection{Introduction}

A detailed analysis of 2D simplified configurations and 3D geometries including the downstream section of a combustion can and the first vane row, has been pointed out in the previous part of the work.

After the former studies, the complexity has been increased leading to the evaluation of thermo-acoustic coupling in a can-annular combustor design.

The focus is on the analysis of the acoustic interaction between the cans.

Simplified 2D and 3D equivalent systems representing the corresponding engine alike turbine design are investigated.

Thermo-acoustic instabilities are reproduced using a forced response approach.

A study of the reflection coefficient and of the transfer function between the cans has been performed.

Comparisons between 2D and 3D equivalent configurations have been estimated.

Another aspect of the current study that also introduces originality compared to previous 


\section{Thermo-acoustic Coupling in Can-Annular Combustors}

work, regards the comparisons performed between different 2D geometries presenting respectively straight and deflecting vanes.

These configurations have been studied also taking into account various distances between the vanes and the wall connecting the two cans.

This study was performed in preparation of a new full engine design with the aim to deliver possible measures to suppress the cross-talk.

\subsection{Investigated configurations}

The cases selected for the analysis are shown in Table 5.1 and 5.2.

For clarity, a nomenclature with initials has been defined for the different geometries analyzed in this part of the work.

The first acronym indicates if the configuration is $2 \mathrm{D}$ or $3 \mathrm{D}$. Then it is specified how many cans the case has: one (1C) or two cans (2C).

The following initial concerns the shape of the vanes: straight vanes (SV) or deflecting vanes (DV).

The last acronym shows which kind of gap has been chosen between the cans and the vanes: reference gap (RG), no gap (NG) or big gap (BG).

The geometry in Fig. 5.1(a) has been taken as reference (RG) and it consists of two neighboring cans $(2 \mathrm{C})$ connected by the stator turbine section modeled with straight vanes (SV).

It is defined as $2 \mathrm{D}-2 \mathrm{C}-\mathrm{SV}-\mathrm{RG}$. Since the width of the can is $d \approx 0.5 \mathrm{~m}$, the ratio between the gap $g$ (space between the vanes and the small wall connecting the two cans) and $d$ can be calculated as $g / d \approx 0.05 / 0.5=0.1$.

The other two different $2 \mathrm{D}$ configurations that have been studied with different vane locations are presented in Fig. 5.1(b) and Fig. 5.1(c).

The case in Fig. 5.1(b) shows no gap (RG) between the vanes and the cans $(g / d \approx 0 / 0.5=0)$, compared with the reference case, and the other one in Fig. 5.1(c) (BG) presents the vanes shifted towards the outlet $(g / d \approx 0.4 / 0.5=0.8)$.

In Fig. 5.2 the reference geometry 2D-2C-SV-RG is presented in order to show the positions of the probes used for analysis in the post-processing and to clarify the directions of the amplitude waves taken into account in the current analysis.

The amplitude wave $A^{+}$represents the pressure excitation imposed at the inlet of the first can through the forced response approach applied in the numerical simulations.

In parallel to these 2D configurations with straight vanes (SV), the complexity has been increased by changing the straight vanes into deflecting vanes (2D-2C-DV) shown in Fig. 5.3, arriving finally to investigate a $3 \mathrm{D}$ case (3D-2C-DV) with two cans and turbine vane section in Fig. 5.5.

The 2D-2C-DV geometry has been analyzed with three different vane locations as RG, NG and $\mathrm{BG}$ as performed for the 2D-2C-SV case.

Before arriving to analyze the 3D-2C-DV geometry, a pseudo-3D test (25D-2C-SV) in Fig. 5.4 has been performed with straight vanes as intermediate step between the 2D-2C geometries and the 3D-2C-DV configurations.

The case 25D-2C-SV is presented as pseudo-3D because it has a resolution in the depth but is just estimated in this direction (Z-axis). 


\begin{tabular}{llll}
\hline Configurations studied & RG & NG & BG \\
\hline 2D-2C-SV & $\sqrt{ }$ & $\sqrt{ }$ & $\sqrt{ }$ \\
25D-2C-SV & $\sqrt{ }$ & no & no \\
2D-2C-DV & $\sqrt{ }$ & $\sqrt{ }$ & $\sqrt{ }$ \\
3D-2C-DV & $\sqrt{ }$ & no & no \\
\hline
\end{tabular}

Table 5.1.: Investigated configurations - Different vane locations

\begin{tabular}{lll}
\hline Configurations studied & Low Mach & High Mach \\
\hline 2D-2C-SV & $\sqrt{ }$ & $\sqrt{ }$ \\
25D-2C-SV & $\sqrt{ }$ & no \\
2D-2C-DV & $\sqrt{ }$ & $\sqrt{ }$ \\
3D-2C-DV & no & $\sqrt{ }$ \\
\hline
\end{tabular}

Table 5.2.: Investigated configurations - Mach conditions

The 25D-2C-SV has been studied with straight vanes and one typology of gap RG.

The results of the $3 \mathrm{D}$ cases have been obtained with deflecting vanes and reference gap (3D2C-DV-RG).

\subsection{Numerical method}

\subsubsection{LES Compressible Solver}

Compressible LES has been applied based on OpenFOAM (section 3.3.1).

More details concerning the turbulence LES models applied have been carried out in section 2.4 .

The duration of the numerical calculations is up to $t=2 \mathrm{~s}$ real time for the $2 \mathrm{D}$ tests.

For the 3D configurations a time step of $\Delta t=1 e-06 \mathrm{~s}$ has been maintained running the calculation for about $200 \mathrm{~ms}$ real time.

\subsubsection{NRBC and Periodicity}

Non-Reflecting Boundary Conditions (NRBC) have been used at the inlet and outlet of the geometries, as already explained in section 2.5 .

Fig. 5.6 shows an overview of the boundaries used for all $2 \mathrm{D}$ and $3 \mathrm{D}$ geometries. As presented in Fig. 5.6 the periodicity has been taken into account and it has been applied downstream of the point where the cans end. 
5. Thermo-acoustic Coupling in Can-Annular Combustors

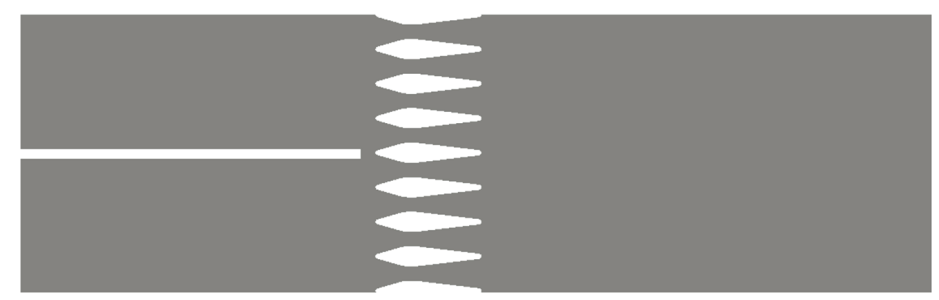

(a) 2D-2C-SV-RG

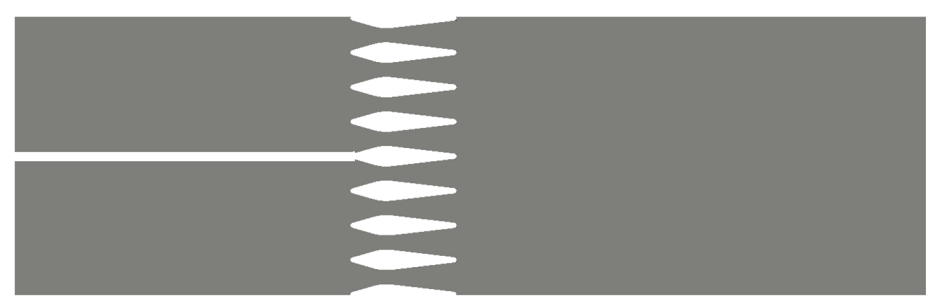

(b) 2D-2C-SV-NG

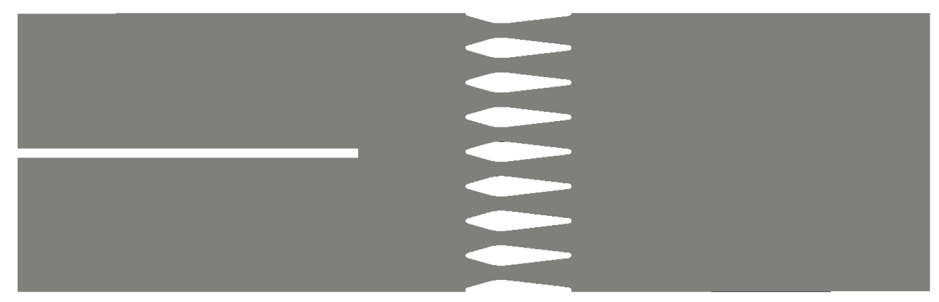

(c) 2D-2C-SV-BG

Figure 5.1.: 2D configurations with SV and different vane locations

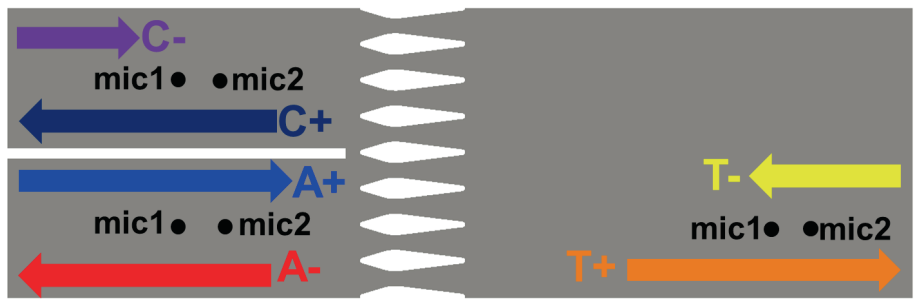

Figure 5.2.: 2D-2C-SV-RG overview with probe positions and waves directions 


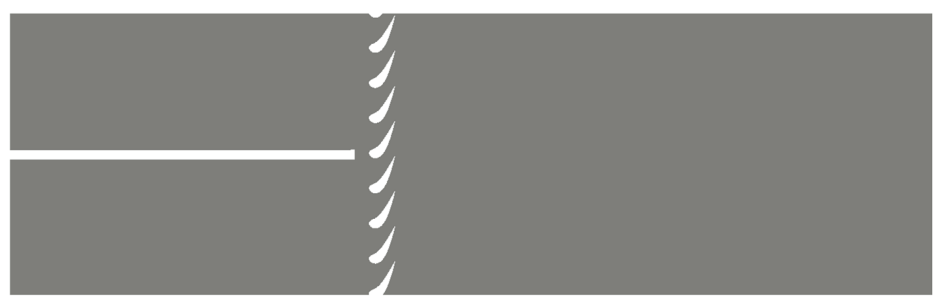

Figure 5.3.: 2D-2C-DV-RG

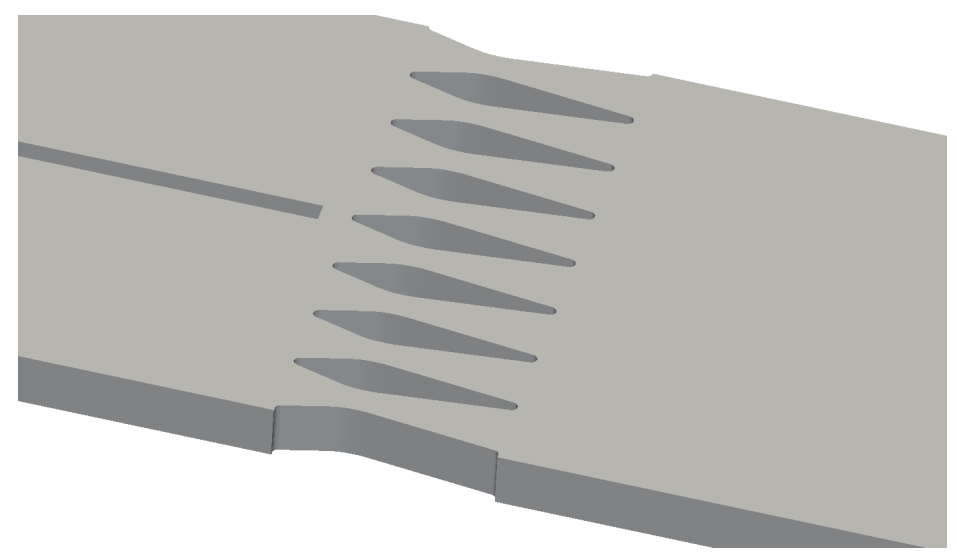

Figure 5.4.: 25D-2C-SV-RG

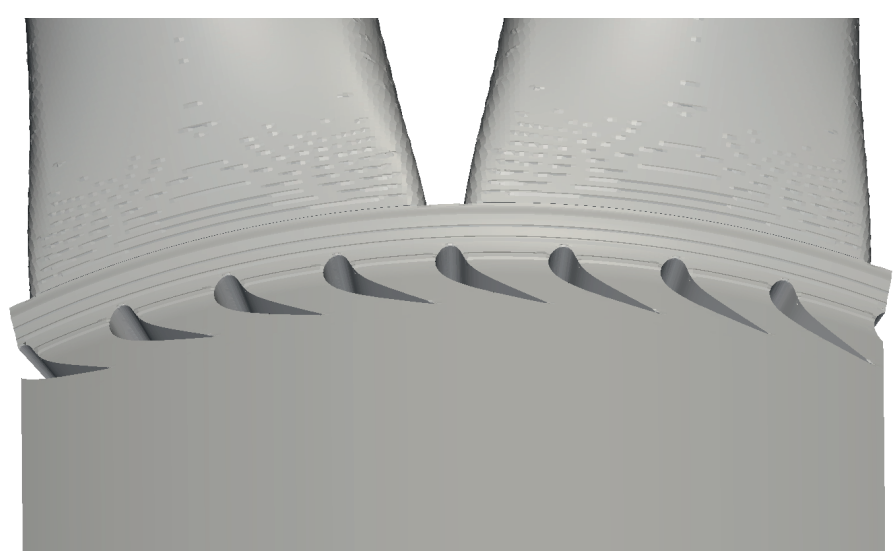

Figure 5.5.: 3D-2C-DV-RG 


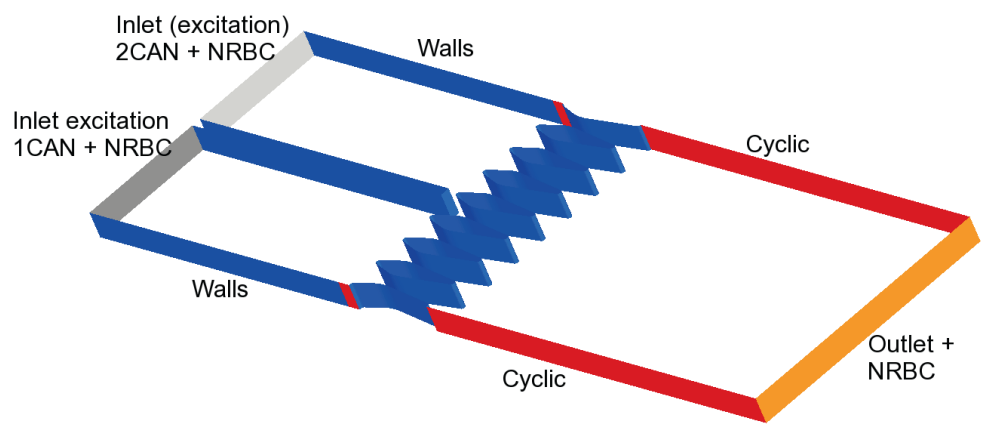

Figure 5.6.: Boundary conditions applied for the cases 2D-2C-SV-RG as example

\subsubsection{Flow}

As explained in section 5.2, the 2D geometries DV and SV have been investigated choosing different positions of the vanes along the duct.

Also different mass flows at the inlet have been set resulting in different Mach numbers chosen as $M \approx 0.4$ and $M \approx 0.7$ in the vane section.

For the $3 \mathrm{D}$ geometries a Mach number $M \approx 0.7$ has been considered (see Table 5.2 ).

\subsubsection{Meshing}

The computational meshes of the 2D configurations analyzed consist of about 128000 cells. The meshes have been generated using the tool BLOCKMESH followed by the mesher SNAPPYHEXMESH.

Refinements have been used around the vanes in order to resolve the gradients with more accuracy.

The mesh for the 3D-2C-DV-RG case shows about 4 million cells and also in this case the tools BLOCKMESH and SNAPPyHexMesh have been applied.

\subsubsection{Forced response approach}

A forced response approach has been used during the simulations imposing a wave excitation at the inlet of the combustion chamber, see section 4.3.5.

Multi-harmonic excitation with a wide frequency range and with small amplitudes (less than $1 \%$ of the mean values) is applied to stay in the linear range. 


\section{Single side excitation}

For the cases 2D-2C-SV-RG NG BG the first can (see Fig. 5.6) has been excited with frequencies within the range $f=20-2000 \mathrm{~Hz}$ (single side excitation) and with amplitudes smaller than $1 \%$ of the mean values.

Concerning the configurations 2D-2C-DV-RG and 3D-2C-DV-RG, two kinds of excitations have been applied. In the first test only one can has been excited with $f=20-2000 \mathrm{~Hz}$ as in the geometry 2D-2C-SV.

\section{Symmetric excitation}

The 2D-2C-DV-RG and 3D-2C-DV-RG geometries have been also analyzed exciting both cans in phase and with the same frequency in the range $f=20-2000 \mathrm{~Hz}$ (symmetric excitation) and with the small amplitudes applied also for the single side excitation case.

For this type of excitation no cross-talk is expected since there is no pressure gradient at the can connection.

\subsubsection{Post-processing - Methods applied}

Different methods have been used next to each other for the post-processing (described in section 2.6.1 and section 2.6.3).

The post-processing is performed using the multi-microphone method (MMM) in order to calculate the transmission and reflection coefficients taking into account the effects of the mean flow.

An amplitude evaluation method based on a results average in the frequency domain and the two-microphone method have also been applied as a first evaluation of the numerical results. In these last methods the quality of the results can deteriorate but they are faster to compute compared to the multi-microphone method.

For this reason they have been used just for a first result check.

The MMM has been used in order to confirm and analyze the results with higher accuracy.

\subsection{Results}

The reflection $A^{-} / A^{+}=R$ and transmission $C^{+} / A^{+}=X$ coefficients obtained from the numerical simulations have been analyzed for all geometries.

Comparisons have been carried out between the 2D and 3D configurations investigated.

\subsubsection{NRBC analysis}

The Non-Reflecting Boundary Conditions applied have been verified at the inlet and outlet evaluating the amplitude waves $T^{-}$and $C^{-}$.

In the ideal case, the result is not influenced by unphysical waves reflected by the NRBC.

In the current cases, the results obtained present an averaged reflection of about 0.2 that does not affect the simulations. 


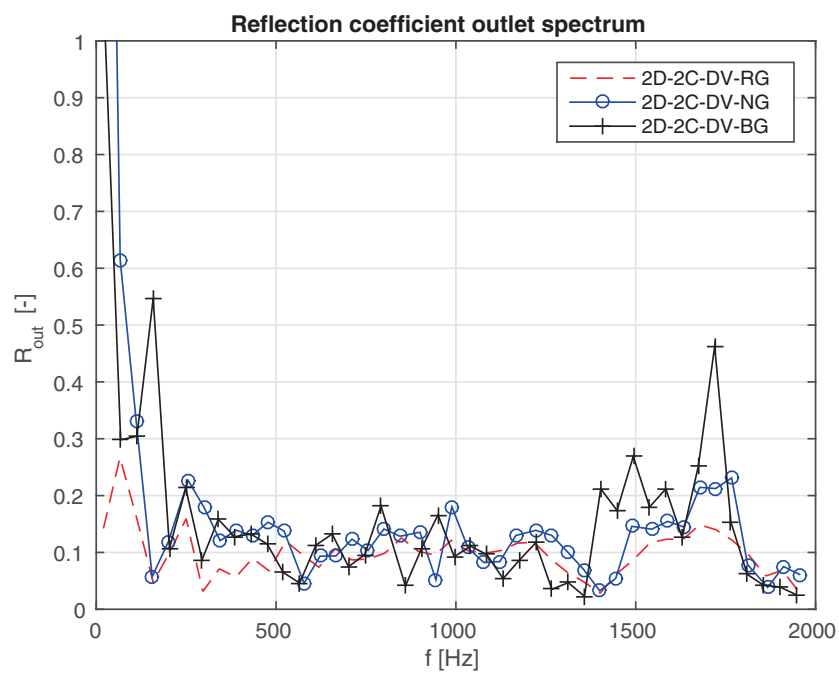

(a) Reflection coefficient spectrum at outlet

Figure 5.7.: Reflection coefficient spectrum at outlet

As an example, Fig. 5.7 shows the reflection coefficient magnitude at the outlet $R_{2}$ for the 2D-2C-DV case with different gaps and $M=0.45$.

All cases show $R_{2}<0.2$ within the range $f=100-1500 \mathrm{~Hz}$.

The case with 2D-2C-DV-BG presents peaks for $f>1600 \mathrm{~Hz}$.

The higher peaks at low frequencies for values of $f<100 \mathrm{~Hz}$ are due to the CFD simulations related to the choice of a relaxation factor in the setting of the NRBC, as discussed in section 2.5 .

\subsubsection{Configuration 2D-2C-SV}

In the diagrams in Fig. 5.8 the ratio between the two wave amplitudes $C^{+} / A^{+}=X$ is shown. The transmission coefficient $X$ represents the percentage of the wave $A^{+}$transferred from the first can to the second one for the 2D-2C-SV configurations with different gaps studied, at Mach numbers of $M \approx 0.4$ and $M \approx 0.7$.

In the plot in Fig. 5.8(a) for the cases with lower Mach, the curve representing the geometry without gap (NG) presents a quite smooth overall behavior with the lowest values of transmission coefficient $X=0.1$ in the range up to $f=630 \mathrm{~Hz}$.

In the same frequency range, the configuration with the vanes moved towards the outlet BG reaches the highest transmission coefficient values of $X=0.8$ as expected. The percentage of the waves that is transferred to the second can is higher in the case with BG compared to the other configurations.

In the range beyond $f=1000 \mathrm{~Hz}$ the same smooth behavior has been predicted for all ge- 


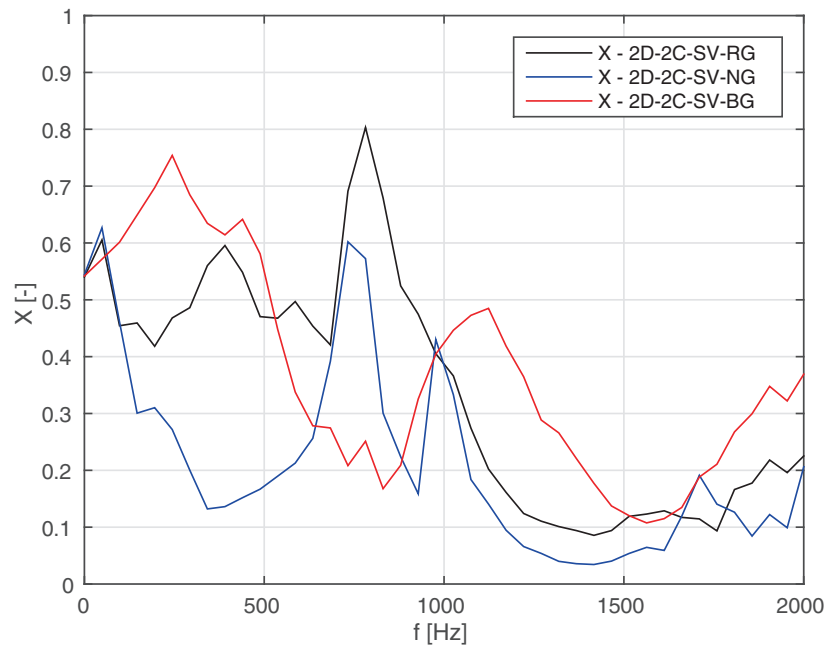

(a) Transmission coefficient $X$ for $2 \mathrm{D}-2 \mathrm{C}-\mathrm{SV}$ with $M \approx 0.4$

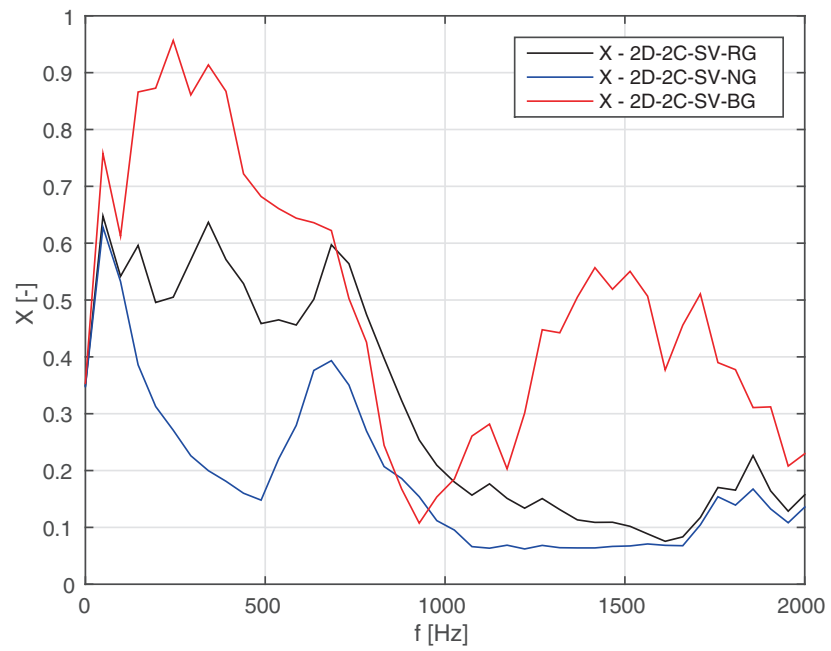

(b) Transmission coefficient $X$ for $2 \mathrm{D}-2 \mathrm{C}-\mathrm{SV}$ with $M \approx 0.7$

Figure 5.8.: Transmission coefficient $X$ evaluation for 2D-2C-SV configurations with different gaps and Mach numbers 


\section{Thermo-acoustic Coupling in Can-Annular Combustors}

ometries with the lowest values of transmission coefficient around $X=0.05$ reached by the case NG matching the expectations.

In the range between $f=630 \mathrm{~Hz}$ and $f=1000 \mathrm{~Hz}$ all geometries show a peak.

The diagram in Fig. 5.8(b) presents the results for the higher Mach number with a quite similar trend compared to the plot shown for lower Mach number.

Here the peak at $f=630 \mathrm{~Hz}$ is less pronounced compared with the case of low Mach.

Hence the acoustic resonance causing the peak is damped by high flow velocities. The geometry with BG reaches the highest values of the transmission coefficient arriving at $X=0.9$.

A reconstruction of the acoustic field has been performed for the 2D-SC-SV-RG case with $M \approx 0.4$ for low and higher frequencies (see Fig. 5.9 and Fig. 5.10).

The idea was to find a common trend between the curve shown in the plot in Fig. 5.8(a) and the reconstruction of the field done on the surfaces.

The script uses a time series of VTK surfaces and performs an FFT for each spacial point on the surface.

More details are pointed out in section 2.6.4.

To better understand the acoustics shown in the plots, a calculation of the cut-off frequency has been performed for each relevant mode.

The results are presented in a diagram in Fig. 5.11.

The values of the cut-off frequencies have been calculated for the width of one can (red dots corresponding to $H \approx 0.5 \mathrm{~m})$ and for the part of the channel after the vanes $(H \approx 1 \mathrm{~m})$.

Since the highest excitation frequency is $f=2000 \mathrm{~Hz}$, the modes that can propagate along the can are of first and second order, as expected and confirmed also by the pressure behavior in Fig. 5.9(a) and Fig. 5.9(b).

In Fig. 5.10(a) and Fig. 5.10(b) transversal modes are visible along the channel downstream the vanes and in the non-excited can.

The plane waves excited at the inlet of the first can be clearly observed and their shape starts to change when they hit the vanes.

Right before the vanes a part of the waves is directed into the gap leading to the second can. This can is excited from both sides since the boundaries are set as periodic.

This side excitation explains the presence of higher modes in the second can.

A conversion of plane waves in the excited can into transversal modes in the passive can take place.

In Fig. 5.12 the transmission coefficient has been presented for the 25D-2C-SV-RG geometry with straight vanes compared with the corresponding $2 \mathrm{D}-2 \mathrm{C}-\mathrm{SV}-\mathrm{RG}$ case with straight vanes and reference gap.

The 25D-2C-SV-RG configuration presents similar behavior compared to the case 2D-2C-SVRG showing clearly also the resonance peak.

This trend confirms the match between $2 \mathrm{D}$ and $3 \mathrm{D}$ equivalent configurations justifying the use of $2 \mathrm{D}$ systems for general analysis saving CPU time.

\subsubsection{Configuration 2D-2C-DV}

In parallel to the plots presented for the configuration with straight vanes $2 \mathrm{D}-2 \mathrm{C}-\mathrm{SV}-\mathrm{RG}$, the acoustic field has been computed also for the reference geometry with deflecting vanes 2D-2C-DV-RG in Fig. 5.13 and Fig. 5.14 for different frequencies within a similar range to 

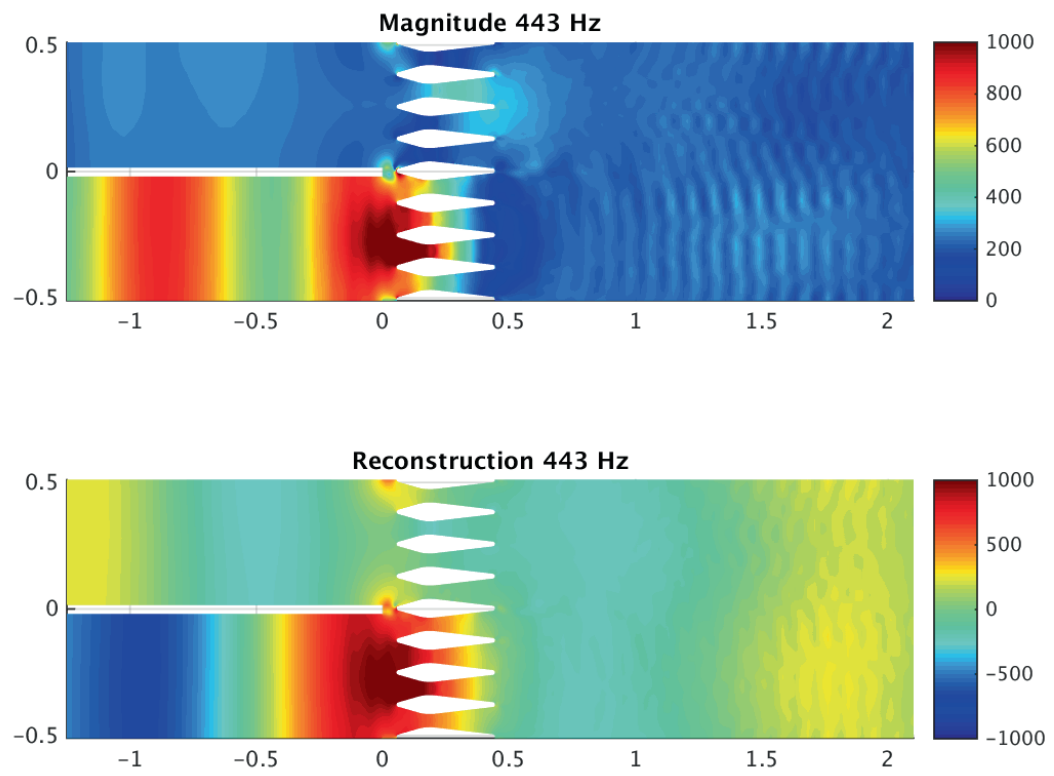

(a) Acoustic field reconstruction at $f=443 \mathrm{~Hz}$ for $2 \mathrm{D}-2 \mathrm{C}-\mathrm{SV}-\mathrm{RG}$
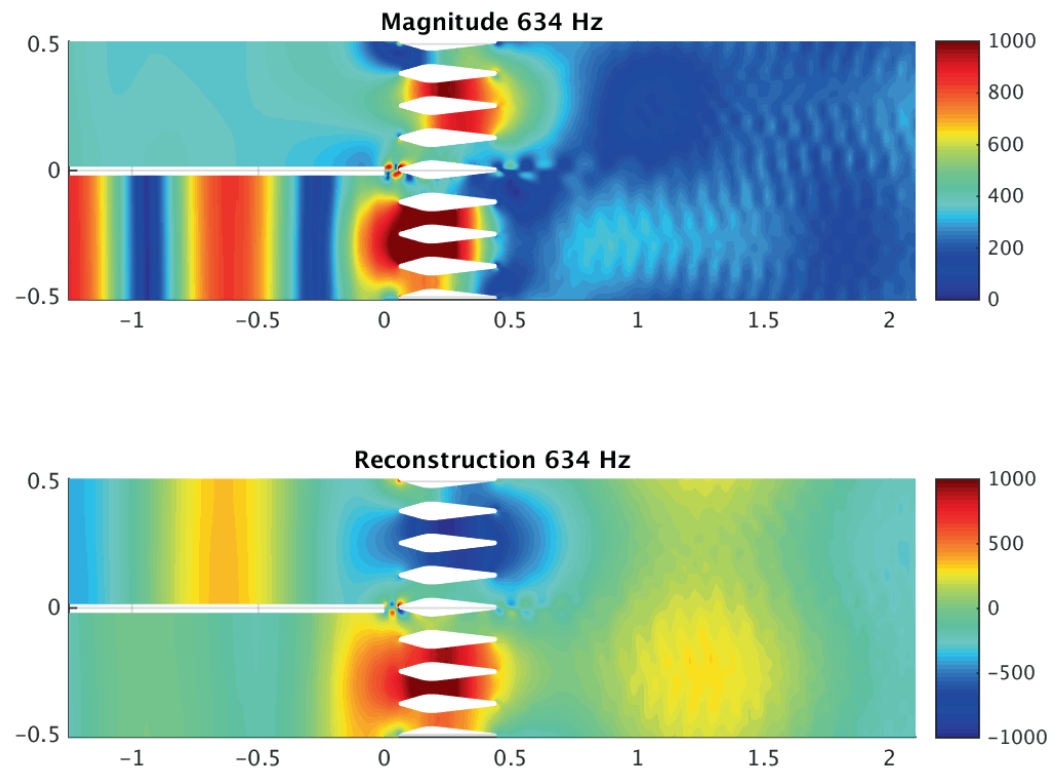

(b) Acoustic field reconstruction at $f=634 \mathrm{~Hz}$ for $2 \mathrm{D}-2 \mathrm{C}-\mathrm{SV}-\mathrm{RG}$

Figure 5.9.: Acoustic field reconstruction for 2D-2C-SV-RG with low frequencies and $M \approx 0.4$ 
5. Thermo-acoustic Coupling in Can-Annular Combustors
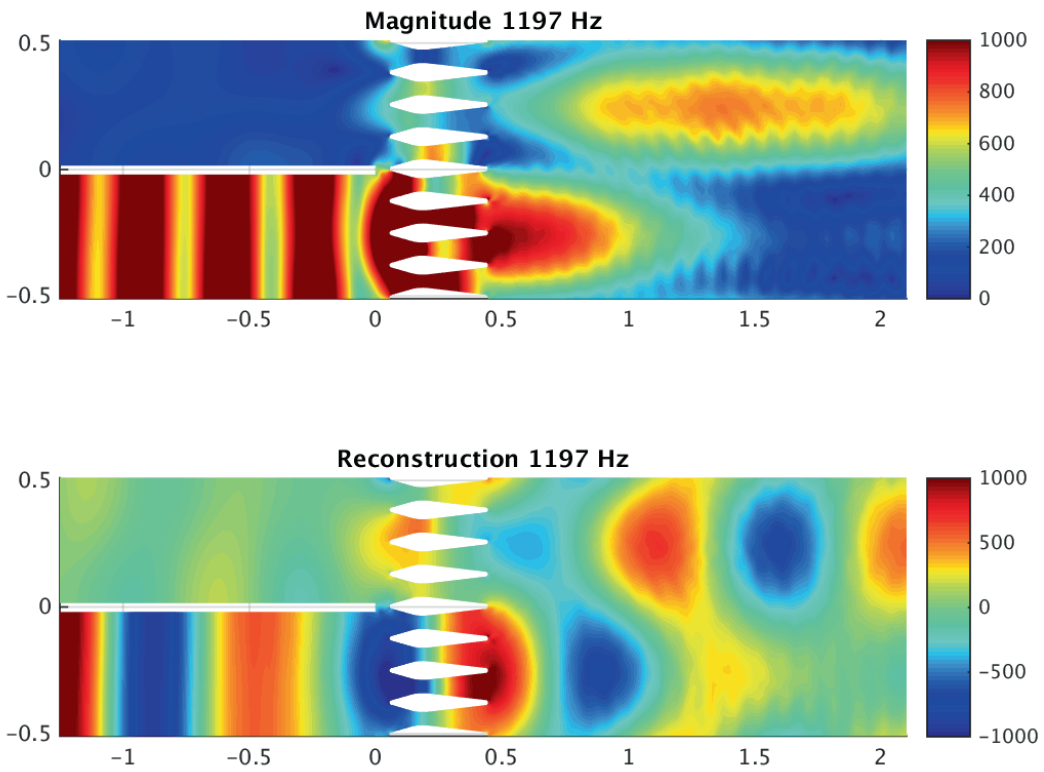

(a) Acoustic field reconstruction at $f=1197 \mathrm{~Hz}$ for 2D-2C-SV-RG
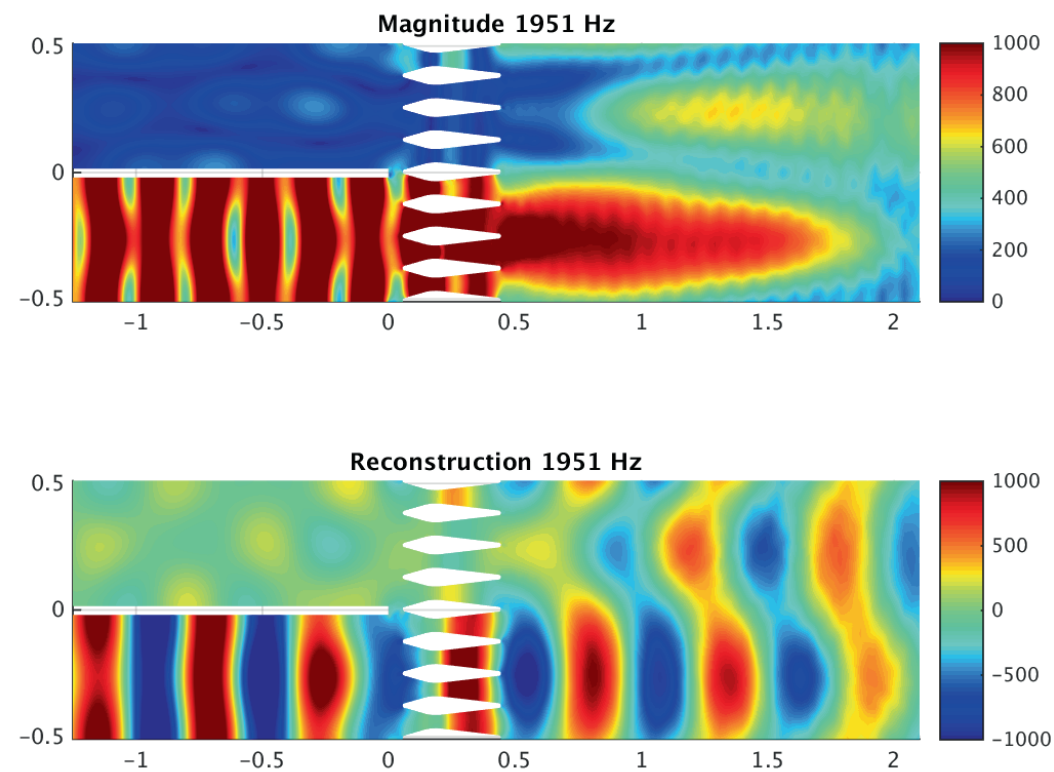

(b) Acoustic field reconstruction at $f=1951 \mathrm{~Hz}$ for 2D-2C-SV-RG

Figure 5.10.: Acoustic field reconstruction for $2 \mathrm{D}-2 \mathrm{C}-\mathrm{SV}-\mathrm{RG}$ with high frequencies and $M \approx 0.4$ 


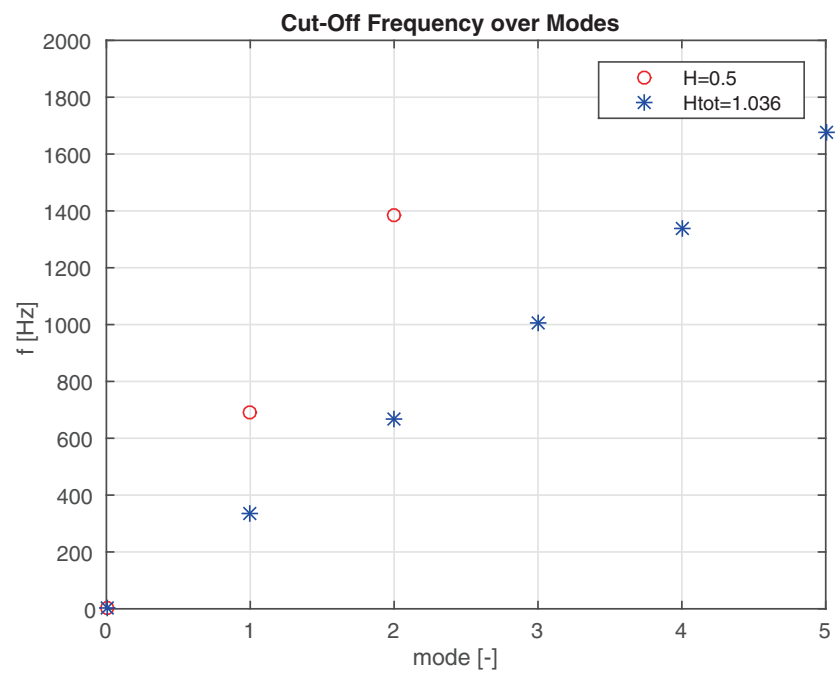

Figure 5.11.: Cut-off frequency plotted over modes

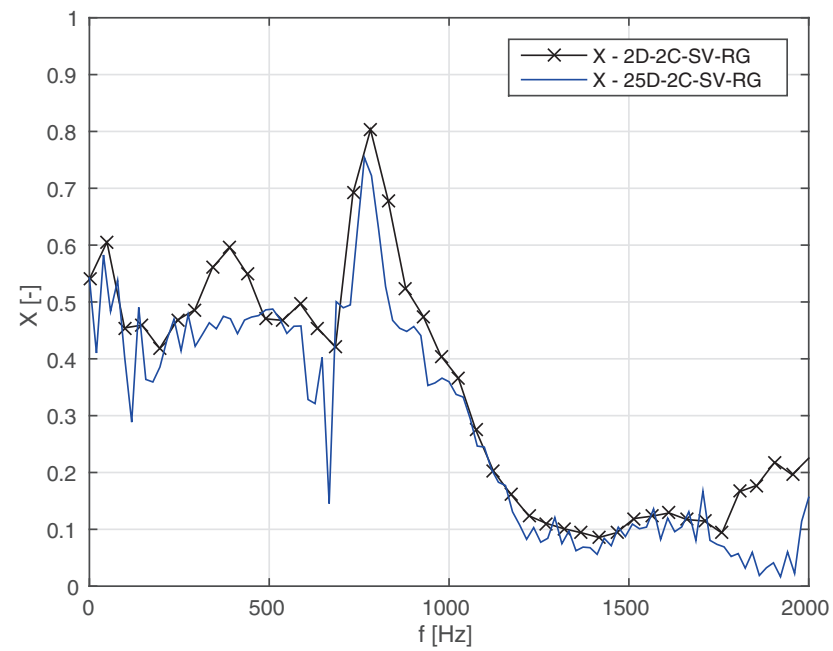

Figure 5.12.: Transmission coefficient $X$ for 25D-2C-SV-RG compared with the corresponding 2D-2C-SV-RG with $M \approx 0.4$ 


\section{Thermo-acoustic Coupling in Can-Annular Combustors}

the previous case with straight vanes.

The plots presenting the pressure field for the highest frequencies for both cases with SV in Fig. 5.10(a), Fig. 5.10(b) and DV in Fig. 5.14(a), Fig. 5.14(b) clearly show in the wider duct the presence of high modes more prominently compared to the images with lower frequencies. In these cases with higher frequencies the higher modes seem to be in part propagated and transferred to the second can.

In Fig. 5.15 the transmission coefficient is presented for the 2D-2C-DV comparing the results obtained for different vane locations.

From the Fig. 5.15 it is evident how the resonance peak disappears for these configurations with deflecting vanes.

The transmission coefficient for the geometry without gap (NG) presents the lowest values matching the expectations.

The case with bigger gap (BG) reaches the highest values compared to the other configurations especially for lower frequencies.

\subsubsection{Configuration 3D-2C-DV with single side excitation}

Concerning the transmission coefficient analysis, numerical results obtained for 3D-2C-DVRG with single side excitation are presented in Fig. 5.16.

The numerical results for the 3D geometry have been compared with the 2D-2C-DV-RG correspondent configuration.

The results indicate an accurate agreement between the 2D and 3D cases, showing a similar behavior of transmission coefficient magnitude and phase. The phase has been calculated choosing the boundary along the section where the cans connect with the vanes.

The transmission coefficient magnitude shows the highest values in the range of low frequencies and starts to decay fast above $f=1000 \mathrm{~Hz}$. Due to the presence of transversal higher modes for higher frequencies beyond about $f=1000 \mathrm{~Hz}$, the post-processing method applied becomes less accurate.

The acoustic field has been calculated also for the configuration 3D-2C-DV-RG for different frequencies (see Fig. 5.17 and Fig. 5.18).

The pressure field observed for the highest frequency in Fig. 5.18(a) and Fig. 5.18(b) underlines the presence of high modes in a clearer way compared to the plots with lower frequencies in Fig. 5.17(a) and Fig. 5.17(b). The Fig. 5.18(a) and Fig. 5.18(b) show how the planar waves along the first can excited start to modify their shape in the region right before the vanes presenting the propagation of higher modes also along the non-excited can.

\subsubsection{Configuration 3D-DV with symmetric excitation}

In this section the numerical results obtained for the case in Fig. 5.19(a) (3D-1C-DV-RG with excitation at inlet of $f=20-2000 \mathrm{~Hz}$ ) are compared with the geometry 3D-2C-DV-RG with same symmetric excitation in Fig. 5.19(b).

Both geometries should theoretically present similar behavior of reflection coefficient (see Fig. 5.20).

The Fig. 5.20 confirms what has been expected. The reflection coefficient magnitude presents a similar trend for both geometries maintaining a constant value of about $R=0.6$ up to 

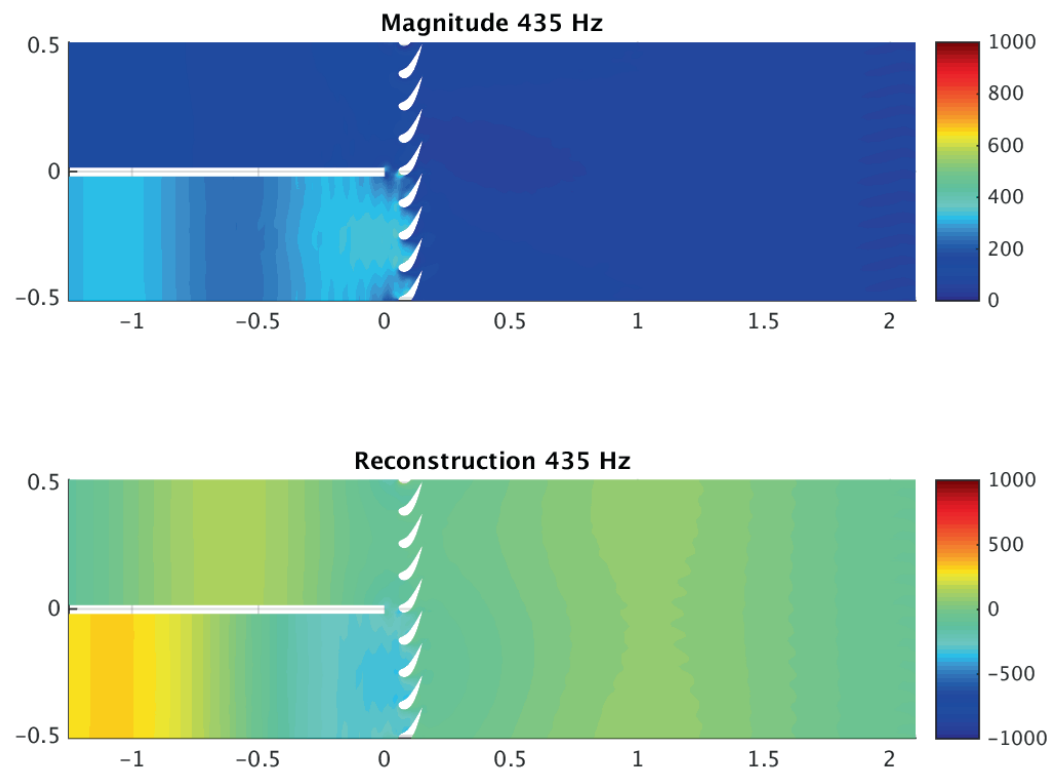

(a) Acoustic field reconstruction at $f=435 \mathrm{~Hz}$ for $2 \mathrm{D}-2 \mathrm{C}-\mathrm{DV}-\mathrm{RG}$
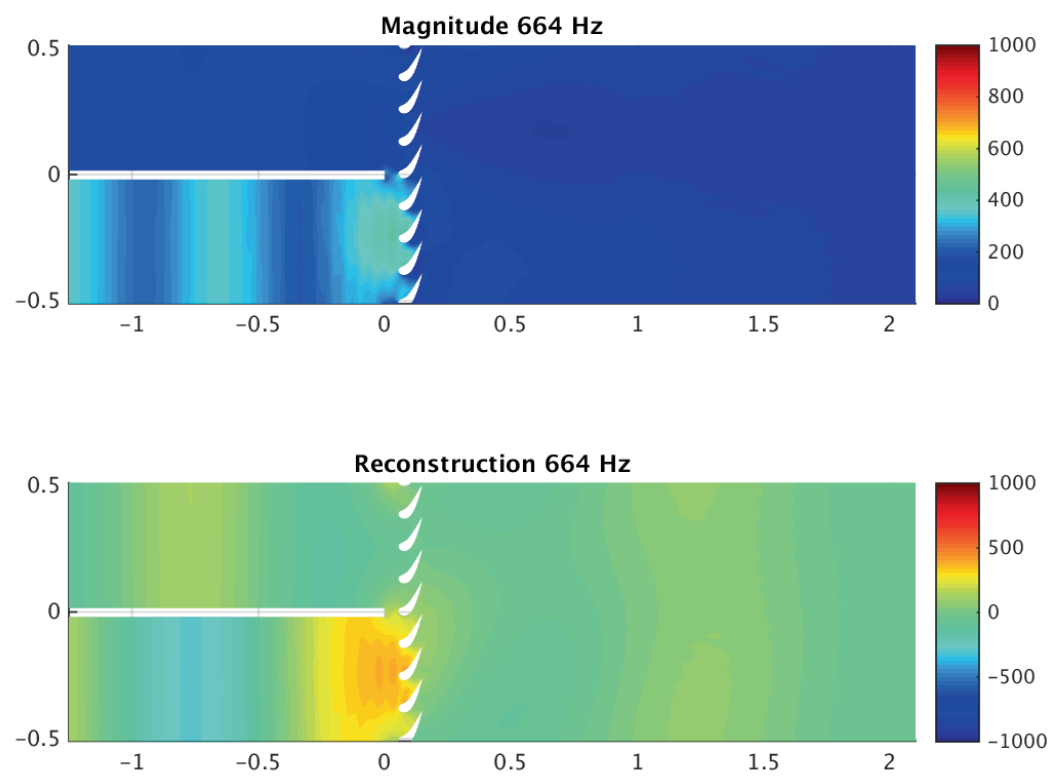

(b) Acoustic field reconstruction at $f=664 \mathrm{~Hz}$ for $2 \mathrm{D}-2 \mathrm{C}-\mathrm{DV}-\mathrm{RG}$

Figure 5.13.: Acoustic field reconstruction for $2 \mathrm{D}-2 \mathrm{C}-\mathrm{DV}-\mathrm{RG}$ with low frequencies and $M \approx 0.45$ 
5. Thermo-acoustic Coupling in Can-Annular Combustors
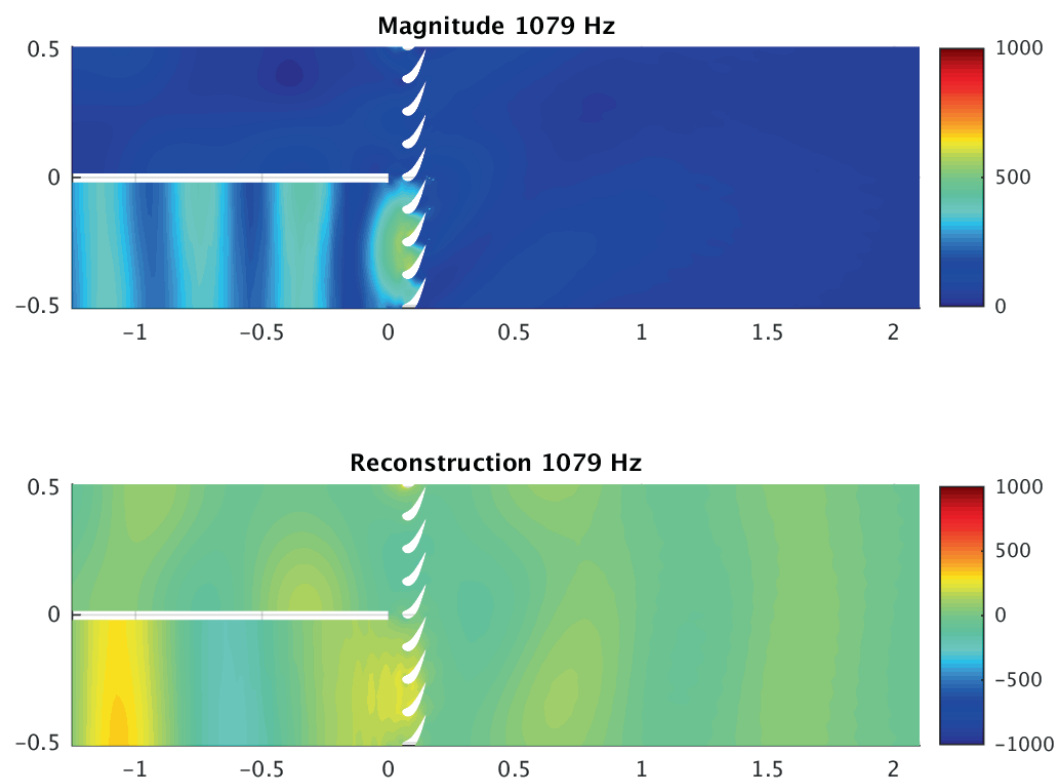

(a) Acoustic field reconstruction at $f=1079 \mathrm{~Hz}$ for $2 \mathrm{D}-2 \mathrm{C}-\mathrm{DV}-\mathrm{RG}$
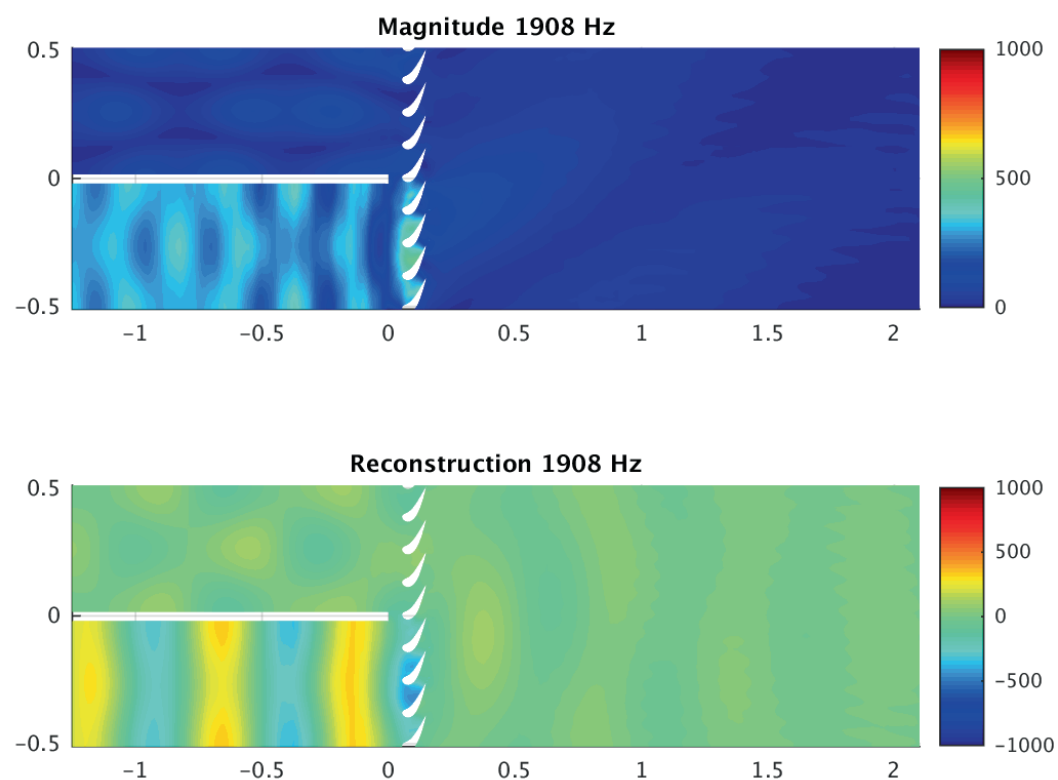

(b) Acoustic field reconstruction at $f=1908 \mathrm{~Hz}$ for $2 \mathrm{D}-2 \mathrm{C}-\mathrm{DV}-\mathrm{RG}$

Figure 5.14.: Acoustic field reconstruction for $2 \mathrm{D}-2 \mathrm{C}-\mathrm{DV}-\mathrm{RG}$ with high frequencies and $M \approx 0.45$ 


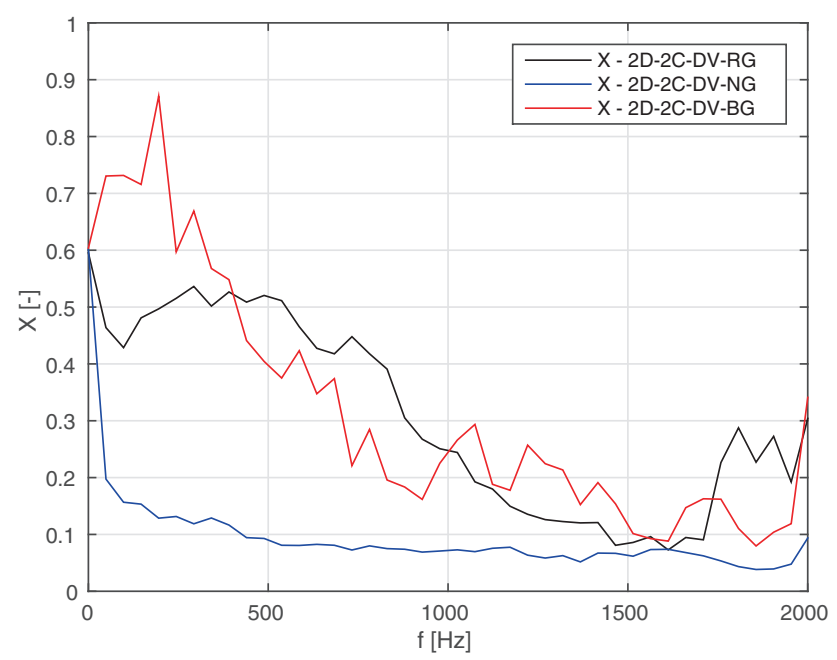

Figure 5.15.: Transmission coefficient $X$ for 2D-2C-DV with different gaps and $M=0.45$

$f=1000 \mathrm{~Hz}$. Above $f=1000 \mathrm{~Hz}$ the values decrease, pointing out the drop of accuracy shown by the method used as observed before.

In Fig. 5.21 the reflection coefficient behavior obtained for the configuration with one can 3D1C-DV-RG has been compared with the geometry 3D-2C-DV-RG for case of no flow. Both geometries present the same trend with reflection coefficient values that are increasing also for frequencies beyond $f=1000 \mathrm{~Hz}$. This demonstrates how the flow can influence also the acoustics.

The acoustic field has been shown for different frequencies (see Fig. 5.22 and Fig. 5.23). The symmetric excitation with same phase applied to both cans appears clearly from the images for all frequencies analyzed.

\subsubsection{Resonance study}

Several studies have been performed in order to determine the reason of the presence of the resonance peak in the configurations with straight vanes in Fig. 5.1.

Calculations with the same flow but without excitation at the inlet have been done in order to verify the validity of the numerical results without external excitation applied. Also in that case the same peak occurred giving the necessity to investigate it more accurately.

A simulation with same flow and external excitation applied at a frequency of $f=630 \mathrm{~Hz}$ within the resonance range has been performed next. The wavelength corresponding to the analyzed frequency $f=630 \mathrm{~Hz}$ has been calculated as $\lambda=825 / 630=1.3 \mathrm{~m}$.

Through this result it can be observed that each wave at that frequency fits in one can. Half wave length of $\lambda / 2 \approx 0.4 \mathrm{~m}$ corresponds to the length of the straight vane and it has been evaluated as $\lambda / 2=826 / 1000 / 2=0.4 \mathrm{~m}$, where $f=1000 \mathrm{~Hz}$ is the highest excited frequency 


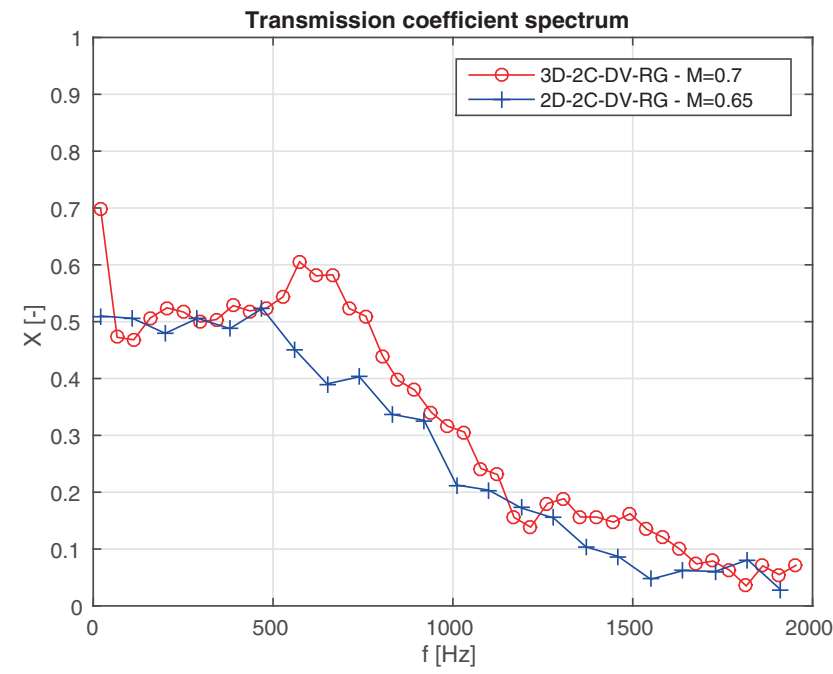

(a) Transmission coefficient magnitude comparisons - $M=0.7$ for 3D-2C-DVRG and $M=0.65$ for 2D-2C-DV-RG

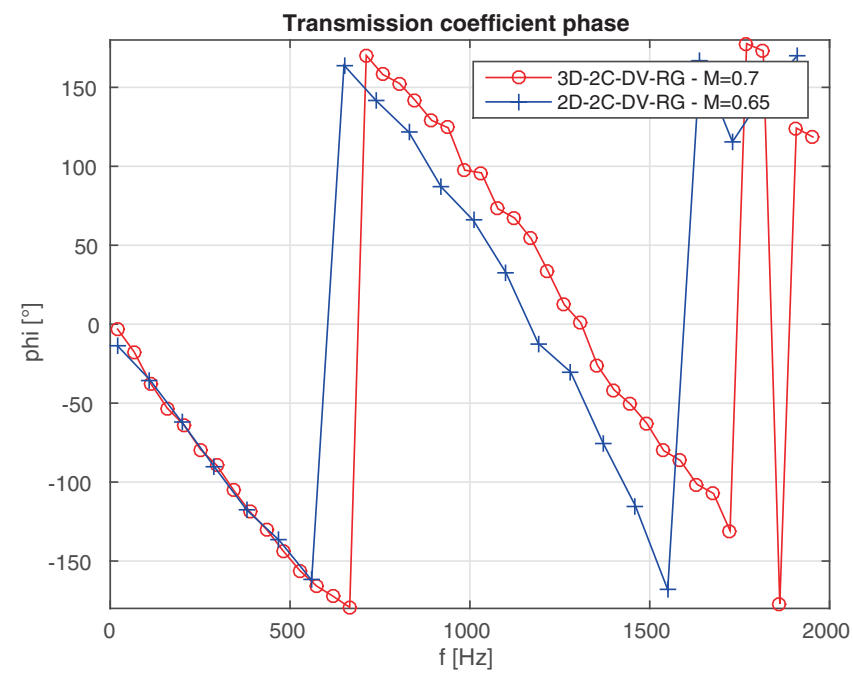

(b) Transmission coefficient phase comparisons - $M=0.7$ for 3D-2C-DV-RG and $M=0.65$ for $2 \mathrm{D}-2 \mathrm{C}-\mathrm{DV}-\mathrm{RG}$

Figure 5.16.: Transmission coefficient at the inlet estimated for $3 \mathrm{D}$ and $2 \mathrm{D}$ configurations with single side excitation and $M=0.7$ 

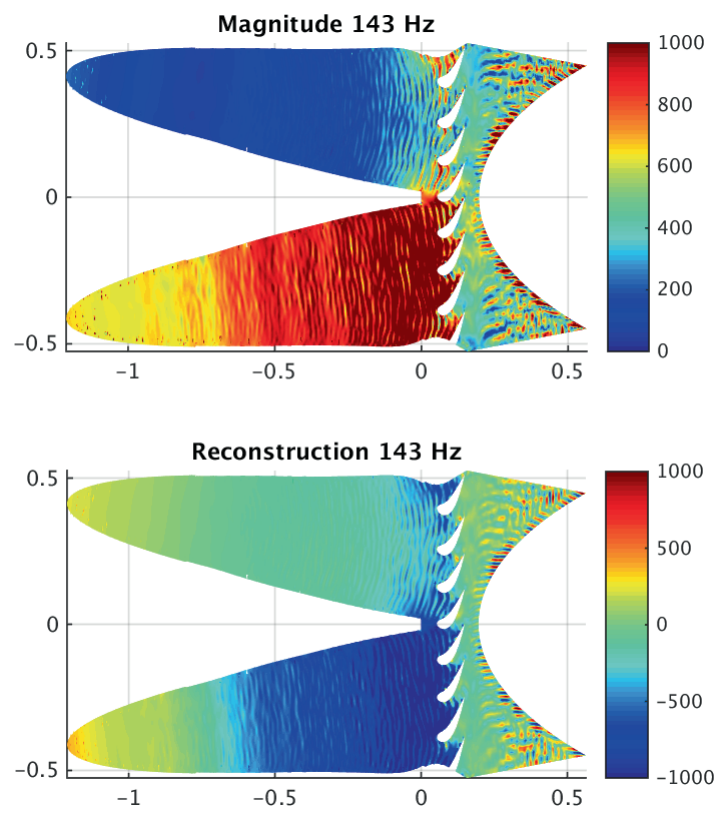

(a) Acoustic field reconstruction at $f=143 \mathrm{~Hz}$ for 3D-2C-DVRG with single side excitation
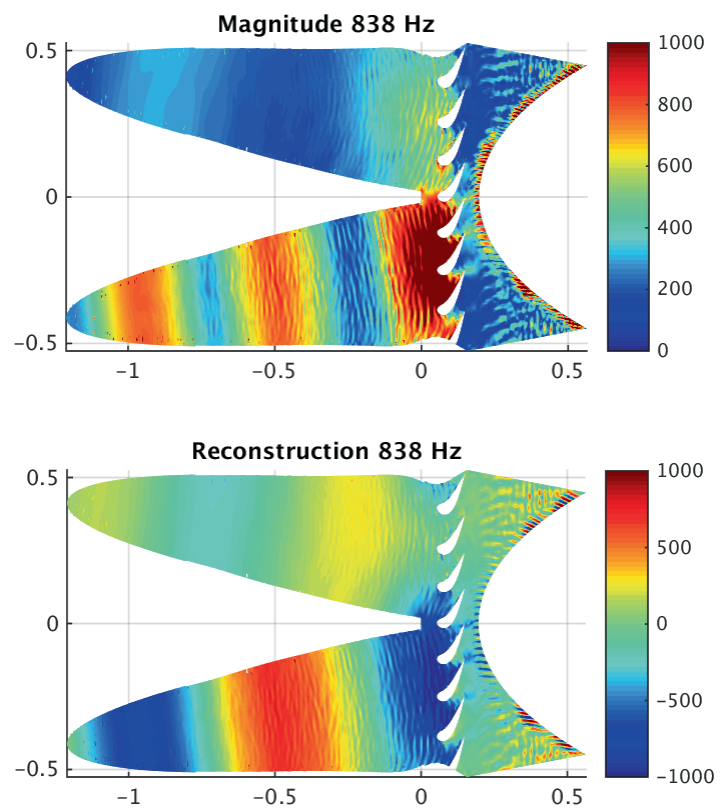

(b) Acoustic field reconstruction at $f=838 \mathrm{~Hz}$ for 3D-2C-DV-

RG with single side excitation

Figure 5.17.: Acoustic field reconstruction at low frequencies for 3D-2C-DV-RG configuration with single side excitation and $M=0.7$ 
5. Thermo-acoustic Coupling in Can-Annular Combustors
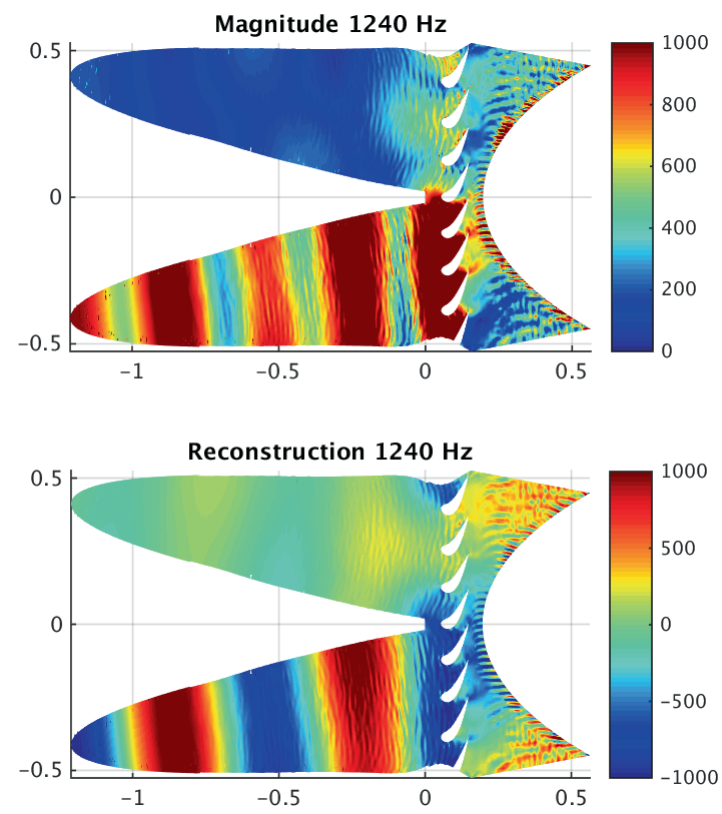

(a) Acoustic field reconstruction at $f=1240 \mathrm{~Hz}$ for 3D-DVRG with single side excitation
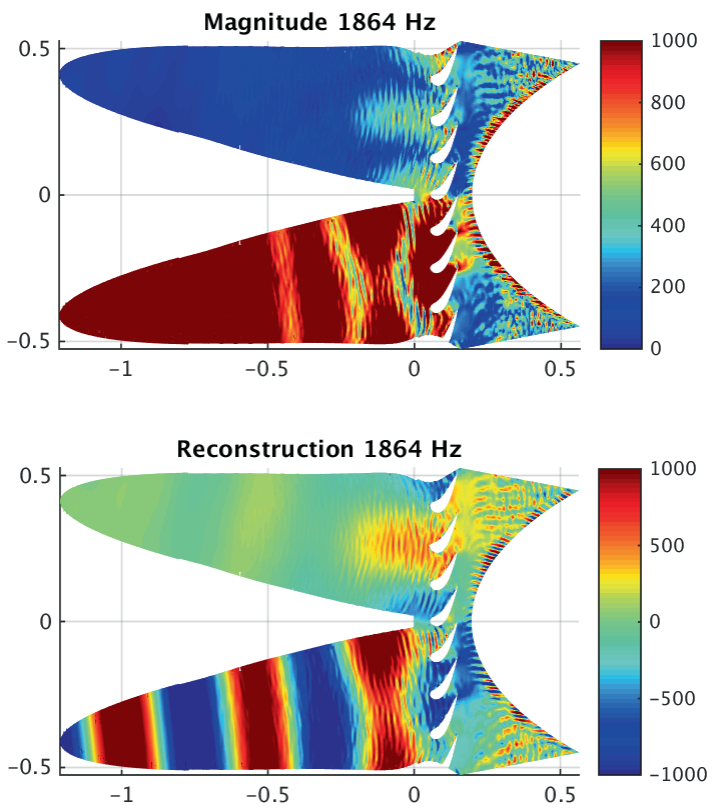

(b) Acoustic field reconstruction at $f=1864 \mathrm{~Hz}$ for 3D-DVRG with single side excitation

Figure 5.18.: Acoustic field reconstruction at high frequencies for 3D-2C-DV-RG configuration with single side excitation and $M=0.7$ 


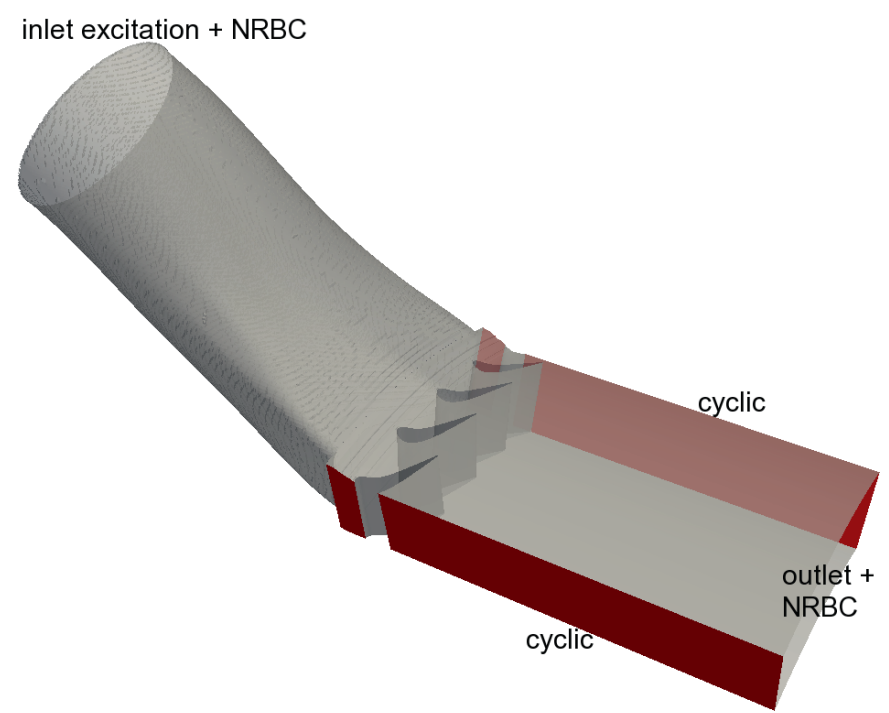

(a) 3D-1C-DV-RG with single excitation

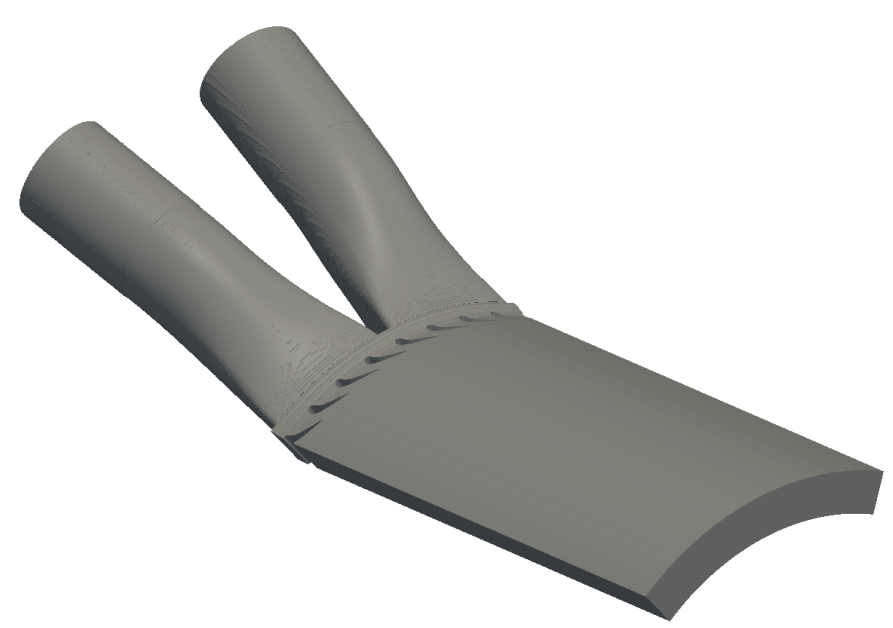

(b) 3D-2C-DV-RG with symmetric excitation

Figure 5.19.: Overview of comparable configurations 


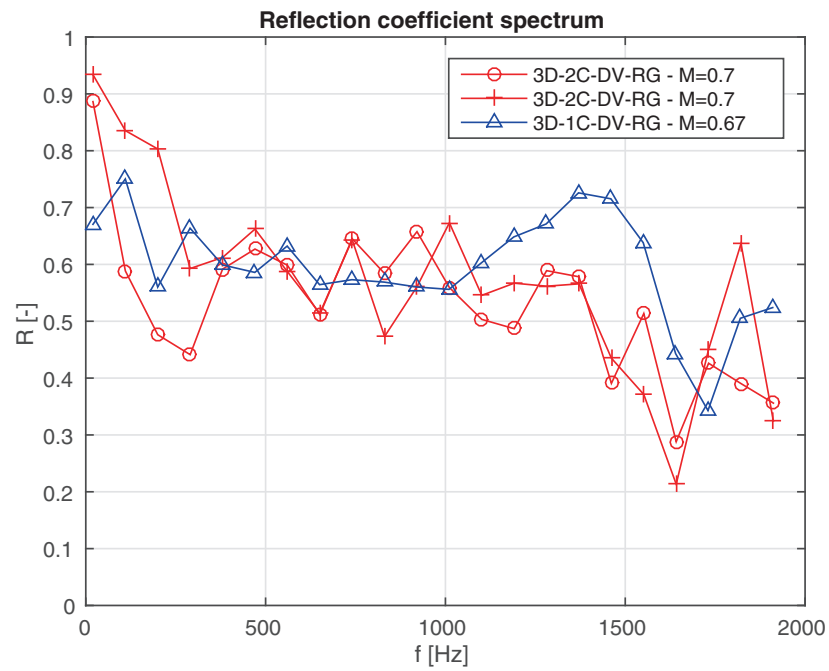

(a) Reflection coefficient magnitude - 3D-1C-DV-RG and 3D-2C-DV-RG comparisons - $M \approx 0.7$

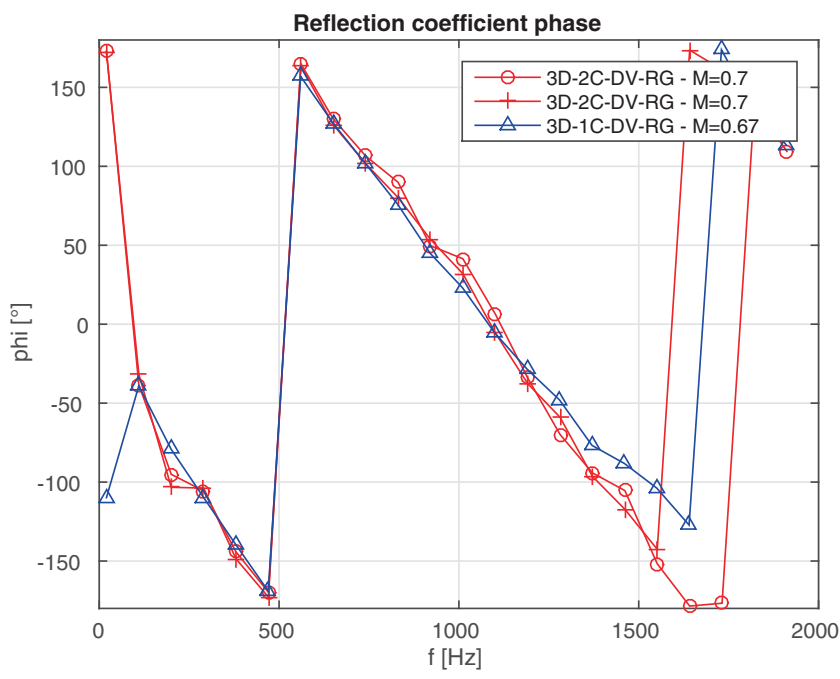

(b) Reflection coefficient phase comparisons - 3D-1C-DV-RG and 3D-2C-DVRG comparisons - $M \approx 0.7$

Figure 5.20.: Reflection coefficient at the inlet estimated for 3D-1C-DV-RG and 3D-2C-DV-RG configurations with symmetric excitation with $M \approx 0.7$ 


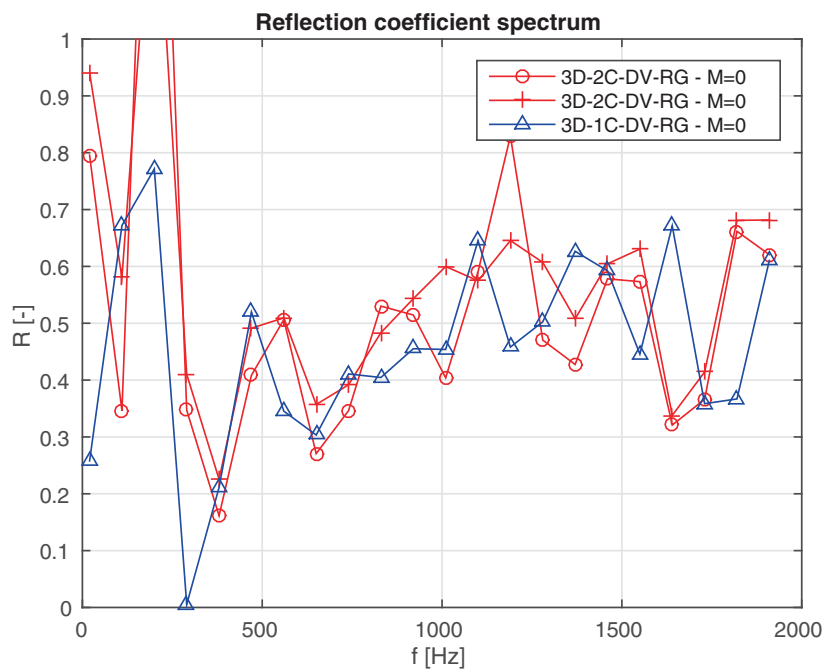

(a) Reflection coefficient magnitude comparisons - 3D-1C-DV-RG and 3D-2CDV-RG comparisons - $M=0$

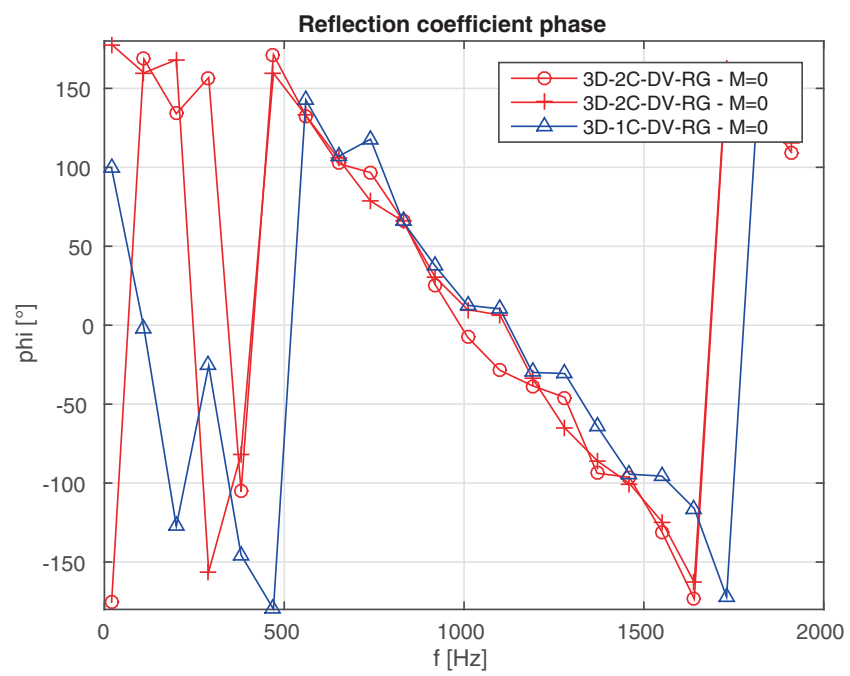

(b) Reflection coefficient phase comparisons - 3D-1C-DV-RG and 3D-2C-DVRG comparisons - $M=0$

Figure 5.21.: Reflection coefficient at the inlet estimated for 3D-1C-DV-RG and 3D-2C-DV-RG configurations with symmetric excitation with $M=0$ 
5. Thermo-acoustic Coupling in Can-Annular Combustors
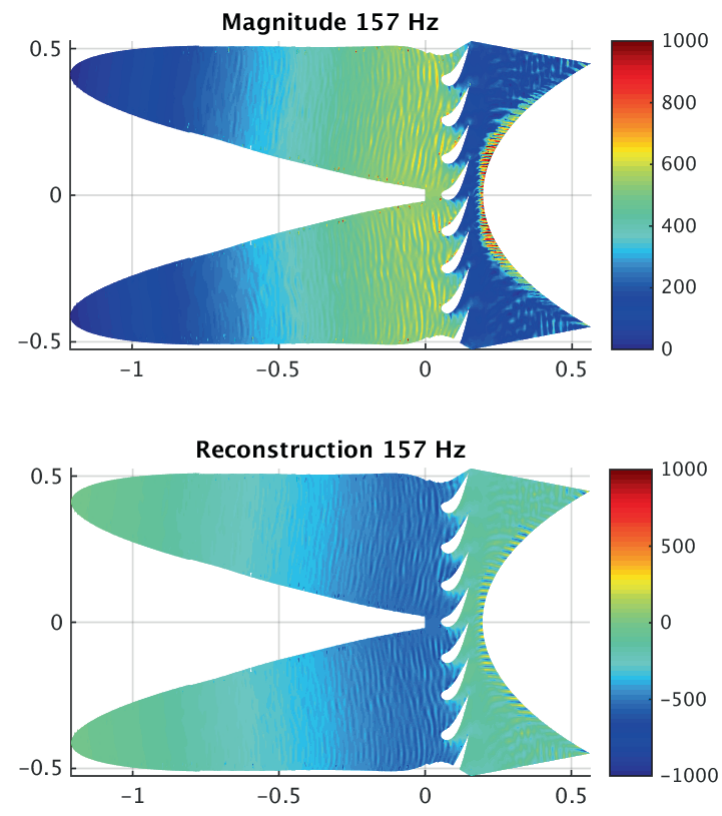

(a) Acoustic field reconstruction at $f=157 \mathrm{~Hz}$ for 3D-2C-DVRG
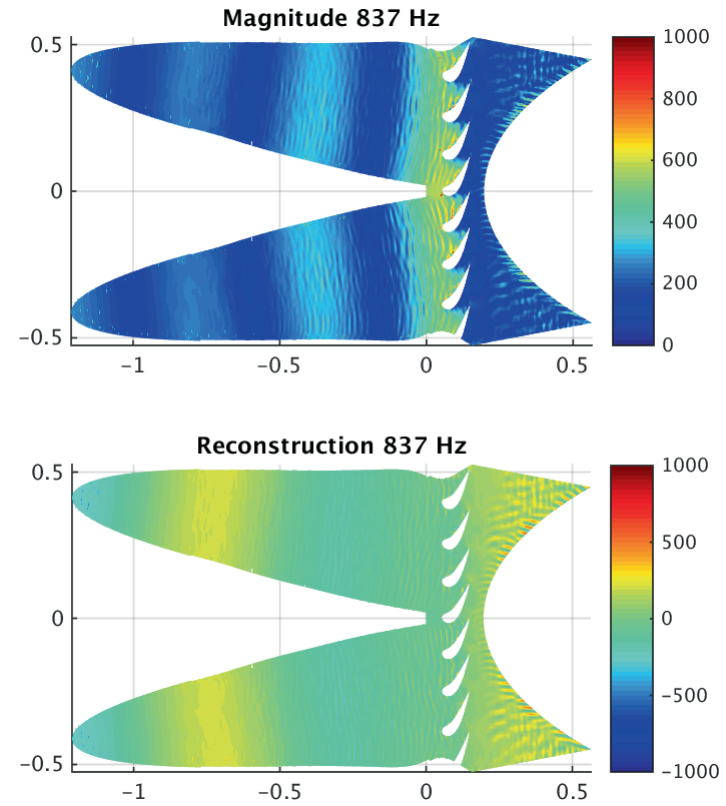

(b) Acoustic field reconstruction at $f=837 \mathrm{~Hz}$ for 3D-2C-DVRG

Figure 5.22.: Acoustic field reconstruction at low frequencies for 3D-DV-RG configuration with symmetric excitation with $M=0.7$ 

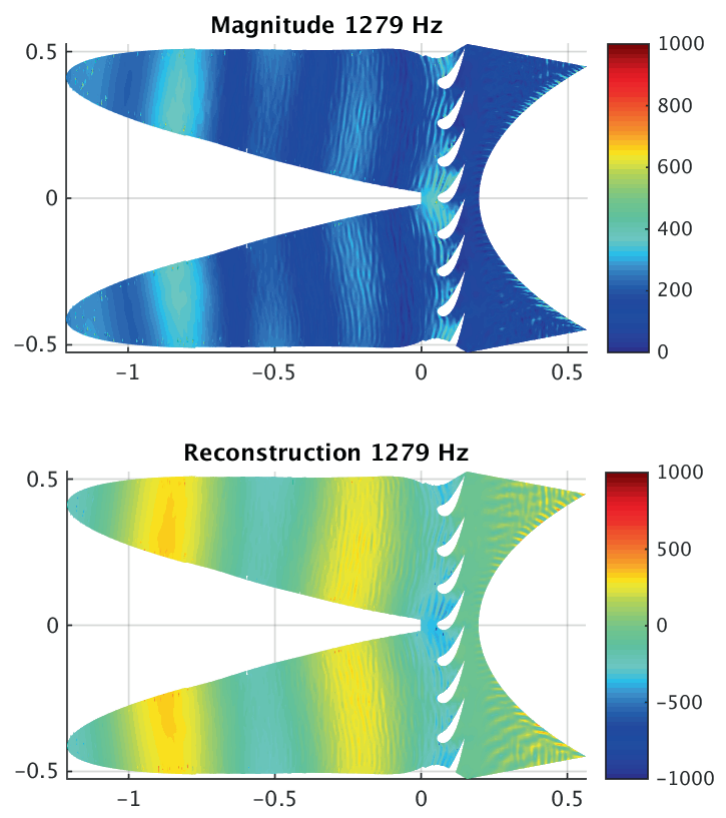

(a) Acoustic field reconstruction at $f=1279 \mathrm{~Hz}$ for 3D-2CDV-RG
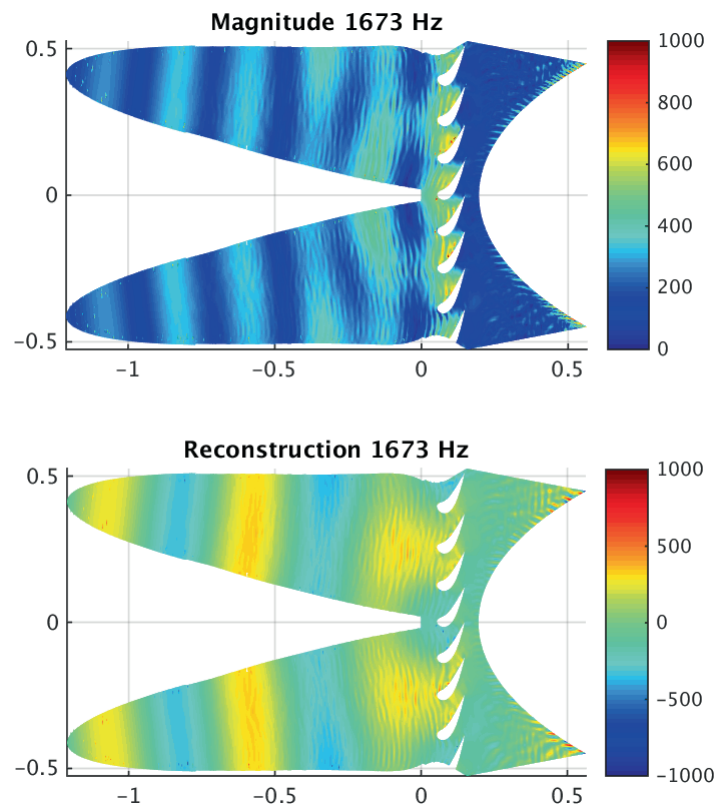

(b) Acoustic field reconstruction at $f=1673 \mathrm{~Hz}$ for 3D-2CDV-RG

Figure 5.23.: Acoustic field reconstruction at high frequencies for 3D-2C-DV-RG configuration with symmetric excitation with $M=0.7$ 


\section{Thermo-acoustic Coupling in Can-Annular Combustors}

within the resonance range. This could cause the presence of the resonance within the range $f=630-1000 \mathrm{~Hz}$.

In order to demonstrate this assumption, the same simulation has been performed with shorter straight vanes with length of $l=0.18 \mathrm{~m}$. This value has been chosen since it represents the chord length of the deflecting vanes studied in this work. The results obtained for the case with deflecting vanes did not show any resonance peak. As expected, the data obtained from the simulation with shorter straight vanes also do not present any resonance.

Fig. 5.24 underlines the lack of the resonance in the case with shorter straight vanes.

The results obtained for the case 2D-2C-SV-RG with single side excitation at $f=630 \mathrm{~Hz}$ can be observed in Fig. 5.25. The pprime parameter represents the pressure field after subtracting the pressure mean values shown in Fig. 5.25(a). The picture presents an interesting behavior of the pressure in the vane region.

In the vanes area periodic oscillations with alternating phase occur. Every second vane seems to oscillate in phase as depicted in Fig. 5.25(b). The plot presents also higher values of amplitudes for the central vanes compared with the values reached by the side vanes.

The plot presented in Fig. 5.26 should check the validity of the simulations. The sum of the amplitude waves $A^{-}$(wave moving back upstream), $C^{+}$(wave transferred to the second can) and $T^{+}$(wave transmitted beyond the vanes) needs to be equal to the amplitude wave $A^{+}$that represents the pressure excitation set at the inlet of the first can through the force response approach applied.

The results satisfy the expectations for the case with deflecting vanes in Fig. 5.26(b).

Fig. 5.26(a) shows the curves obtained for the geometry with straight vanes. The curve representing $A^{+}$reaches lower values compared to the other curve in the range up to $f=$ $1000 \mathrm{~Hz}$.

This mismatch could be due to interference on the results of the resonance peak observed in the plots shown before.

\subsection{Conclusion}

The objective of this work was to analyze the acoustic interaction between combustor cans, verifying measurement results that prove a coupling and synchronization of the pressure modes in neighboring cans.

Cases of increasing complexity have been presented. The CFD open source code OpEnFOAM has been used for the numerical analysis. A forced response approach has been applied imposing a single side wave excitation (only one can excited with $f=20-2000 \mathrm{~Hz}$ ) and symmetric excitation (both cans excited with same frequency range $f=20-2000 \mathrm{~Hz}$ ).

Comparisons have been performed and estimated between 2D and 3D equivalent configurations.

The transmission and reflection coefficient behaviors present an accurate agreement between the $2 \mathrm{D}$ and $3 \mathrm{D}$ cases showing a similar trend within the frequency range analyzed.

For the results obtained for frequencies beyond $f=1000 \mathrm{~Hz}$, it necessary to take into account that the post-processing method applied becomes less accurate. This fact is due to the presence of transversal higher modes for higher frequencies beyond $f=1000 \mathrm{~Hz}$.

In parallel to these geometries, the $2 \mathrm{D}$ cases with straight vanes (2D-2C-SV) and deflecting 


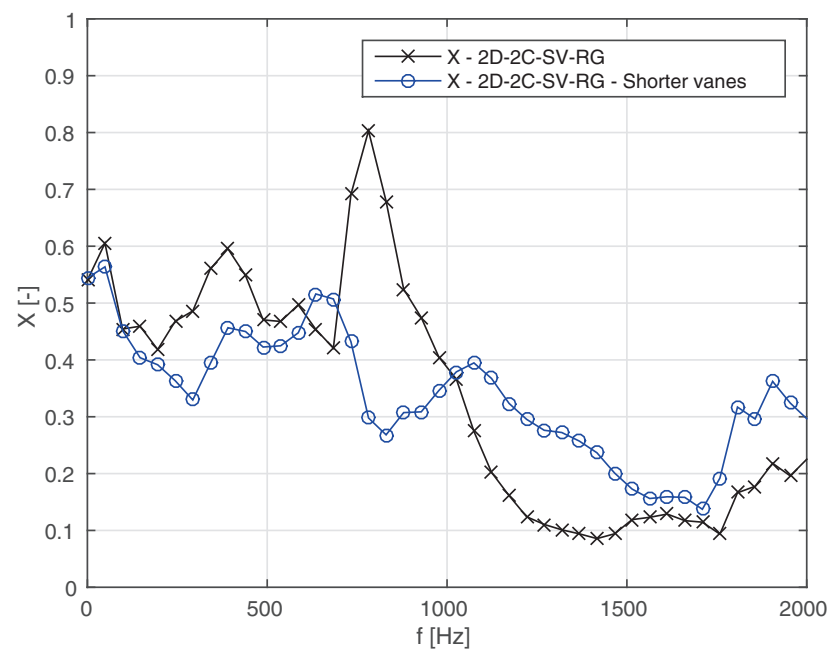

Figure 5.24.: Transmission coefficient comparisons between 2D-2C-SV-RG and 2D-2C-SV-RG with shorter vanes

vanes (2D-2C-DV) have been analyzed also with different vane locations.

The first test called reference gap ( $\mathrm{RG}$ ) presented ratio between the gap $g$ (space between the vanes and the small wall connecting the two cans) and $d \approx 0.5 \mathrm{~m}$ (width of the can) of $g / d \approx 0.05 / 0.5=0.1$. The second case had no gap $(\mathrm{NG})$ between the vanes and the cans $(g / d \approx 0 / 0.5=0)$ and the last one presented bigger gap $(\mathrm{BG})$ with the vanes shifted towards the outlet $(g / d \approx 0.4 / 0.5=0.8)$.

As expected the $2 \mathrm{D}$ configurations studied with deflecting vanes (DV) and with the vanes moved towards the outlet (BG) present the highest values of transmission coefficient of about $X=0.8$ for low frequencies of $f<500 \mathrm{~Hz}$. For higher frequencies up to $f=2000 \mathrm{~Hz}$ the transmission coefficient values decrease reaching 0.1 at about $f=2000 \mathrm{~Hz}$. The case with reference gap (RG) reaches values of $X=0.5$ for $f<500 \mathrm{~Hz}$, arriving to 0.1 at $f=1600 \mathrm{~Hz}$. The geometry with no gap (NG) shows a transmission coefficient with constant value at about $X=0.1$ within the whole frequency range analyzed. The different gaps evaluated with the configuration with straight vanes (SV) show a similar trend observed before in the case with deflecting vanes (DV) and various gaps for low frequencies up to $f=600 \mathrm{~Hz}$. In the range between about $f=630-1100 \mathrm{~Hz}$ they all present a resonance peak that needs further investigation.

It has been proven that the cross-talk cannot be neglected for all kind of gaps here analyzed. Since the case without gap (NG) reaches the lowest values of transmission coefficient of $X=0.1$, a possible solution would be to add in the newest engine design a device (for example a flange) between the vanes and the cans in order to block the passage between the cans. As outlook of this work, it is recommended to have more cans in the test rig with the purpose to obtain the proper acoustic boundary conditions. 
5. Thermo-acoustic Coupling in Can-Annular Combustors

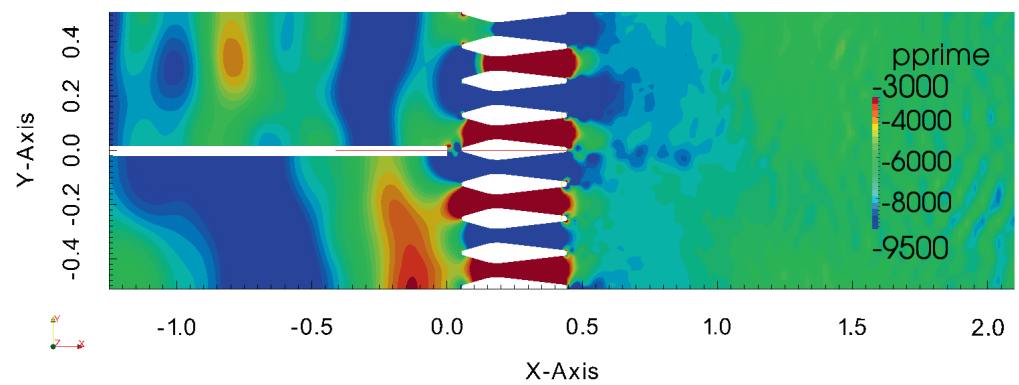

(a) Acoustic pressure field behavior

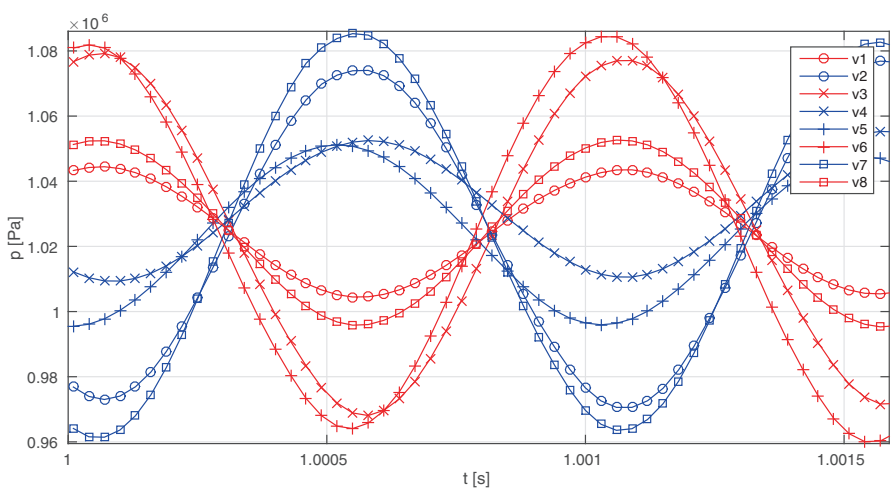

(b) Acoustic waves along the vanes

Figure 5.25.: Pressure evaluation for case 2D-2C-SV-RG with single side excitation at $f=630 \mathrm{~Hz}$ 
5.5. Conclusion

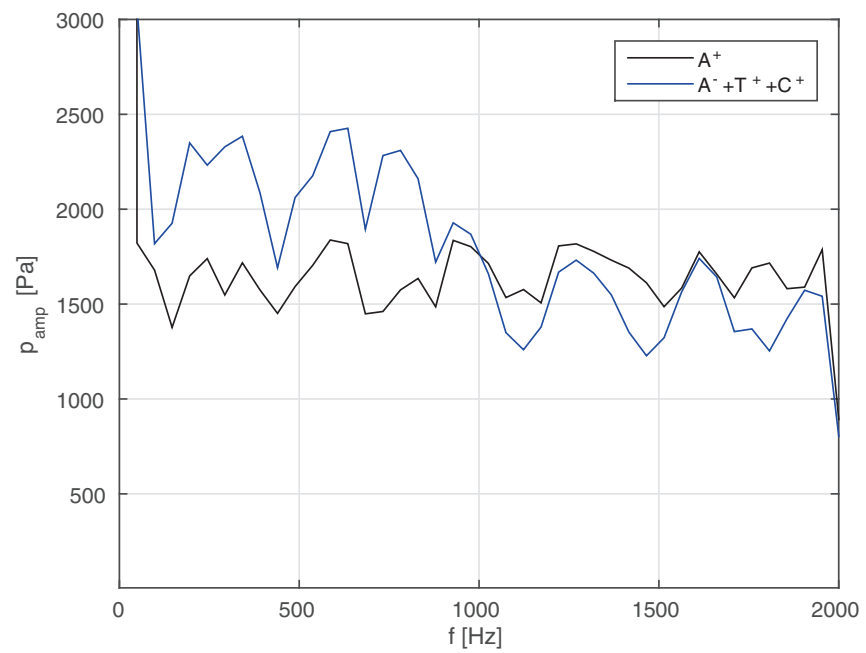

(a) Wave amplitude sum for the 2D-2C-SV-RG

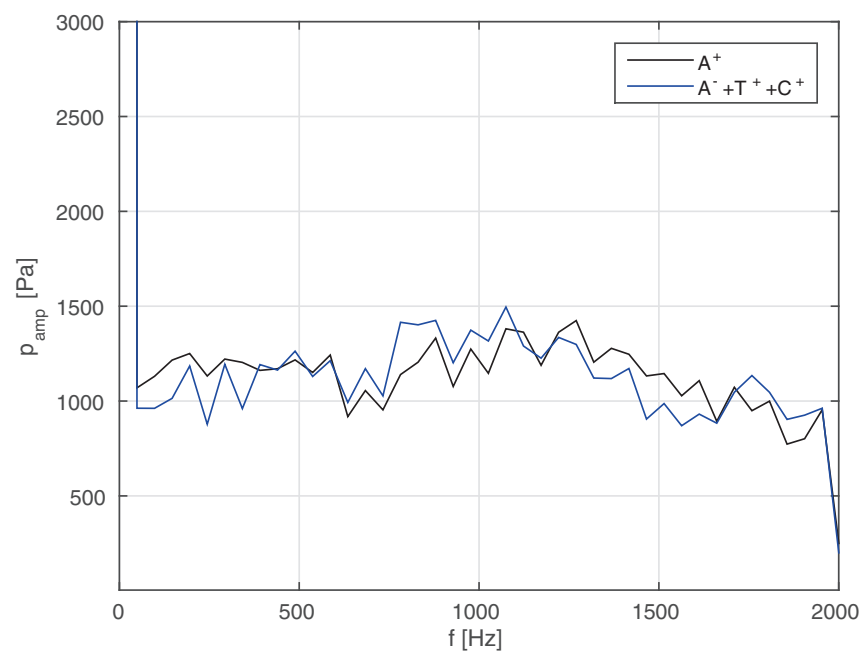

(b) Wave amplitude sum for the 2D-2C-DV-RG

Figure 5.26.: Wave amplitudes evaluation 
5. Thermo-acoustic Coupling in Can-Annular Combustors 


\section{Conclusion and Outlook}

\subsection{General introduction}

For current lean premixed combustion technology, thermoacoustic instabilities became an important issue to solve. Such technologies are a challenging task with the aim to reduce NOx emissions. To evaluate these acoustic instabilities, configurations of different complexity have been presented and analyzed in this dissertation.

\subsection{Thermoacoustic Influence of the Turbine on the Combustor - validation of tool chain}

In the first part, the main aim was to analyze the reflection coefficient at the outlet of the combustion chamber and to validate the numerical results obtained for $2 \mathrm{D}$ geometries by comparing them with analytical formulations.

Compressible LES has been applied based on the open source CFD code OPENFOAM for the numerical analysis. A forced response approach has been used provoking a wave excitation at the inlet of each geometry with a frequency range of $f=20-2000 \mathrm{~Hz}$. The $2 \mathrm{D}$ cases have 


\section{Conclusion and Outlook}

been performed with Non-Reflecting Boundary Conditions (NRBC) set at the inlet and outlet of each geometry. In the post-processing different methods have been tested and described.

The 2D cases analyzed show agreement with the analytical formulations in case of no flow. The cases with flow present the highest values of reflection coefficient compared with no flow tests. The values of the reflection coefficient $R_{2}$ at the outlet of each geometry confirm and verify the reliability of NRBC used. In summary, a tool chain has been established with basic behavior validated.

\subsection{Numerical Simulation of Sound Propagation through the Can-Annular Combustor Exit}

Two models of different complexity have been analyzed in this part of the work. The same numerical method and forced response approach applied for the previous study, have been also used. A detailed study of the reflection coefficient $R$ behavior before the vanes has been performed for the $2 \mathrm{D}$ and $3 \mathrm{D}$ correspondent geometries. The numerical results have been compared with analytical formulae.

In case of no flow the geometries have been compared with the analytical formulation of 1D transfer matrix method. In case of flow the analytical approximation shown in Leyko et al. [8] has been used for comparisons with the numerics.

The numerical results obtained for both $2 \mathrm{D}$ and $3 \mathrm{D}$ configurations carried out an overall reasonable agreement with the analytical behavior.

\subsection{Thermo-acoustic cross-talk between cans in a can-annular combustor}

After the previous studies, the complexity has been increased leading to the evaluation of the acoustic interaction between two neighboring cans. This analysis derives from the need to validate measurements obtained in can-annular combustion chambers that indicated synchronization and oscillation in phase or with opposite phase of pressure modes in neighboring burners. These observations indicate the limits of using a single combustor can in a test rig, to test the acoustics and coupling to combustion dynamics. The single can has different acoustic boundary conditions as compared to a system of coupled cans, and the phenomenon of cross talk is absent in a single can. Theoretically the can-annular combustion chamber should be less vulnerable to transversal waves as compared to the annular design, since the burners are acoustically coupled only through the turbine stator stage and the plenum. The results gained from the experiments show another unexpected behavior of the cans that need to be proved also numerically.

A detailed analysis of 2D configurations and 3D geometries has been performed. Same numerical method used for the previous works, has been also applied for this part. Two different kind of excitations have been evaluated: single side excitation (only one can excited with $f=20-2000 \mathrm{~Hz}$ ) and symmetric excitation (both cans excited with same frequency range 
$f=20-2000 \mathrm{~Hz}$ ). Sampling data from the numerical simulations have been used to perform a post-processing analysis with the multi-microphone method.

Comparisons have been performed and estimated between 2D and 3D equivalent configurations. The $2 \mathrm{D}$ geometries have been investigated with different gaps between the vanes and the cans, with different vane shapes and Mach numbers.

It has been finally proven that the cross-talk cannot be neglected and it has been shown how to reduce it through the choice of different vanes locations. Since the configurations without gap (NG) reach the lowest values of transmission coefficient of $X=0.1$, a possible solution would be to add in the new engine design a device (for example a flange) between the vanes and the cans in order to block the passage between the cans. The expected value of the transmission coefficient for the configurations without gap (NG) would have been of $X=0.0$, but the numerical result obtained of $X=0.1$ could be due to the incomplete accuracy of the NRBC, especially for low frequencies up to about $f=500 \mathrm{~Hz}$. The higher values of the transmission coefficient at low frequencies of $f<500 \mathrm{~Hz}$ are related to the choice of a relaxation factor in the setting of the NRBC, as explained in section 2.5.

\subsubsection{Outlook}

The presence of the resonance peak at $f=630 \mathrm{~Hz}$ shown just for the $2 \mathrm{D}$ configurations with straight vanes, various gaps and different Mach numbers needs further investigation.

It is recommended to have more cans in the test rig with the purpose to obtain the proper acoustic boundary conditions.

\subsection{Characterization of rotor effects on can-annular combustor acoustics}

\subsubsection{Introduction}

In the last part of the work, the rotating components have been included into the complex geometry before analyzed consisting of two neighboring cans and the first turbine vane row. During the investigation of mesh generation a major step to be taken became clear. For the compressible solvers a rotational mesh functionality needs to be constructed. If this is performed in future $\mathrm{PhD}$ research, and combined with the knowledge and tools developed in the present thesis, a full analysis of the acoustics in coupled combustor cans and turbine stages can be possible.

\subsubsection{Motivation for needing a rotating mesh capability - problem description}

The need to take into account rotating meshes represents a main step necessary to run a whole turbine simulation. For this aim, compressible simulations of rotating turbine stage sectors have been performed in order to experience the features as periodicity, rotating meshes and 


\section{Conclusion and Outlook}

sliding interfaces available in the code OpEnFOAM. An analysis of test cases has been done together with performing new tutorials using different versions of the code. The tutorials performed with version OPENFOAM 2.1 and OPENFOAM 1.6ext have been presented with technical details in the Appendix. 


\section{A. Appendix}

\section{A.1. Example of numerical setting applied}

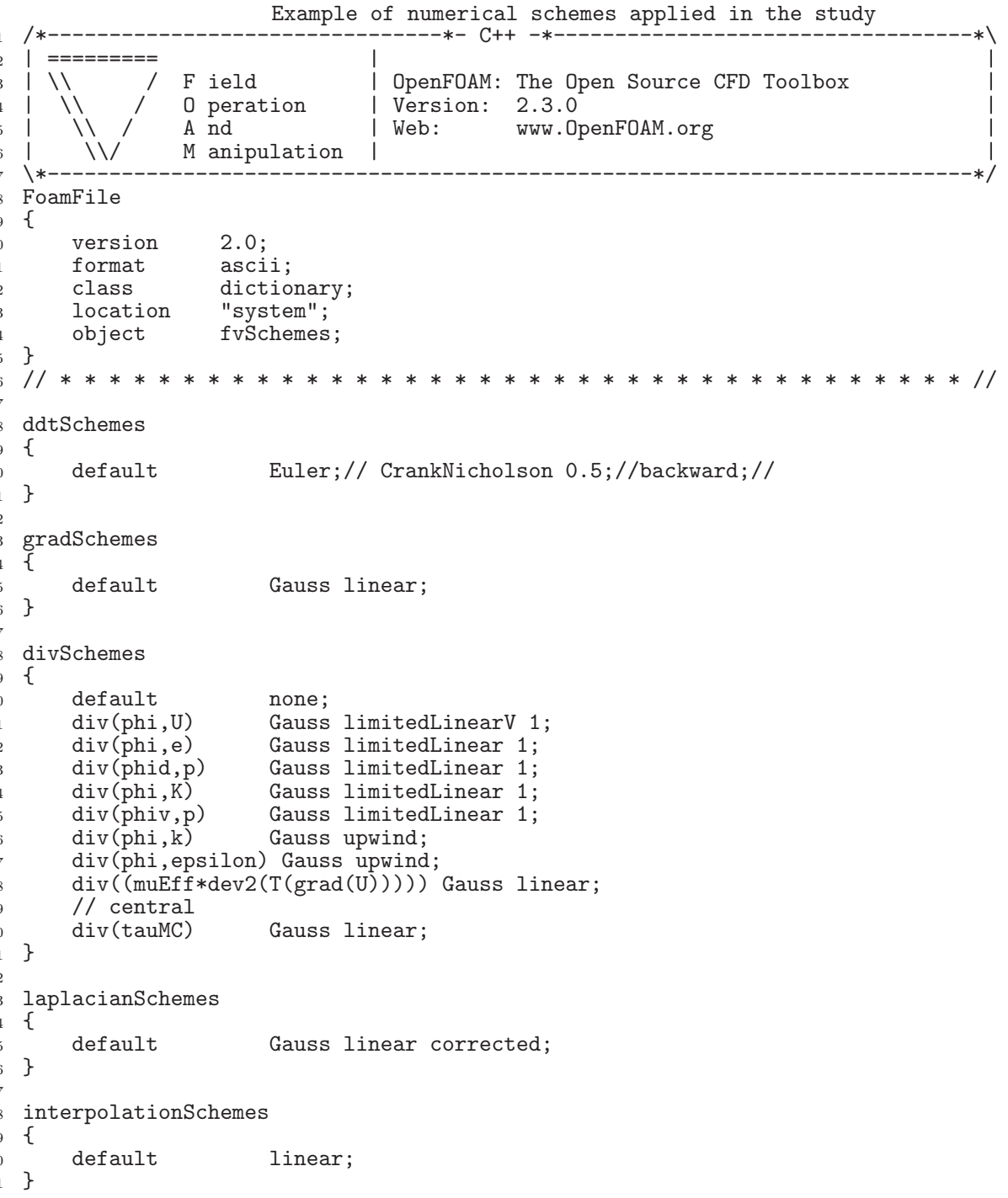


A. Appendix

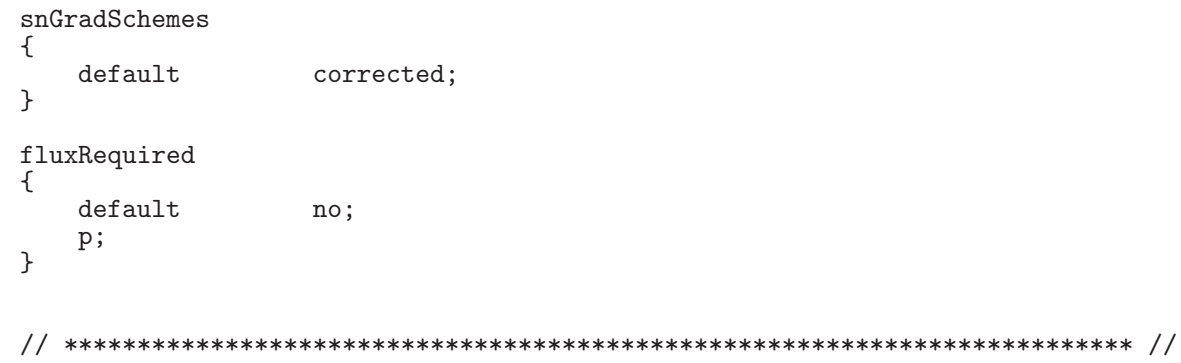

Example of numerical setting used during the simulations

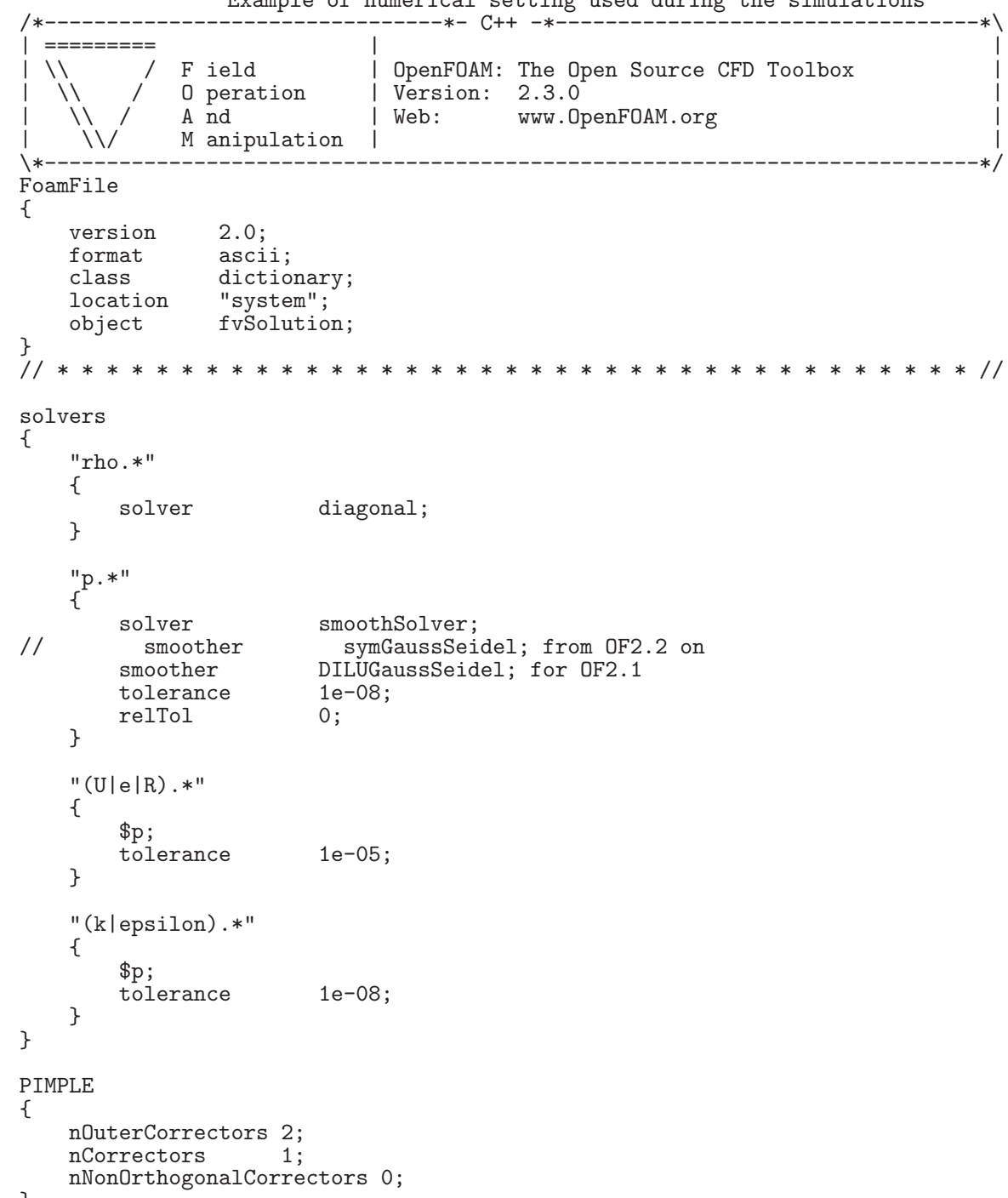




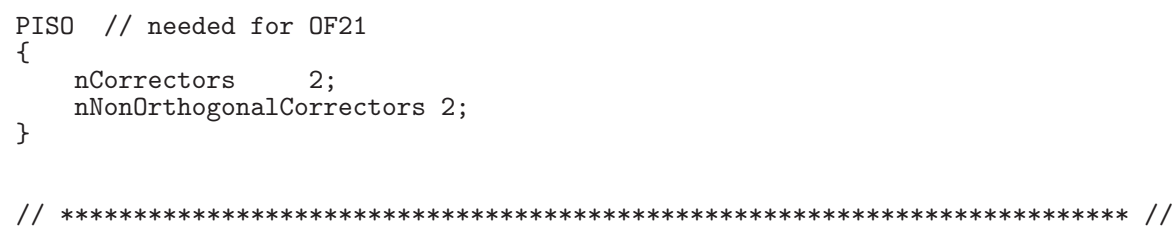

Example of numerical setting for NRBC applied in the study Non-reflecting uniform fluctuating velocity inlet

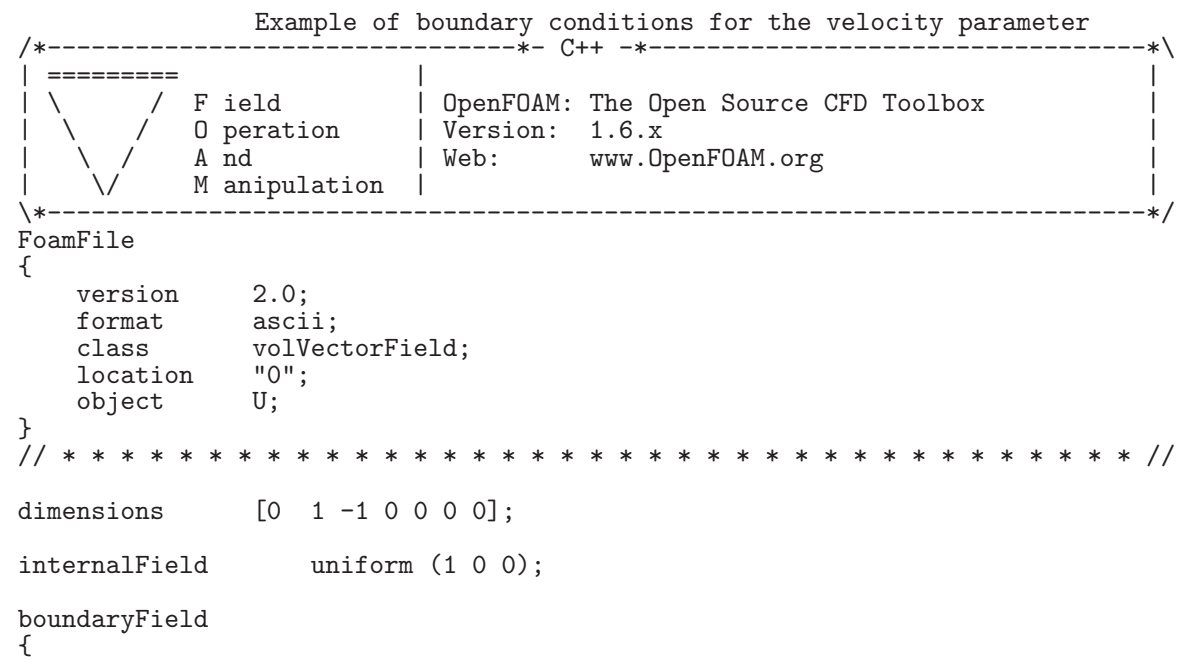


A. Appendix

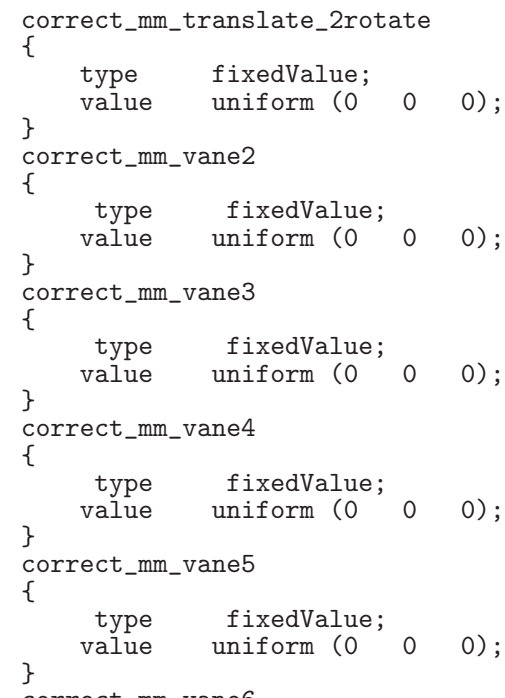




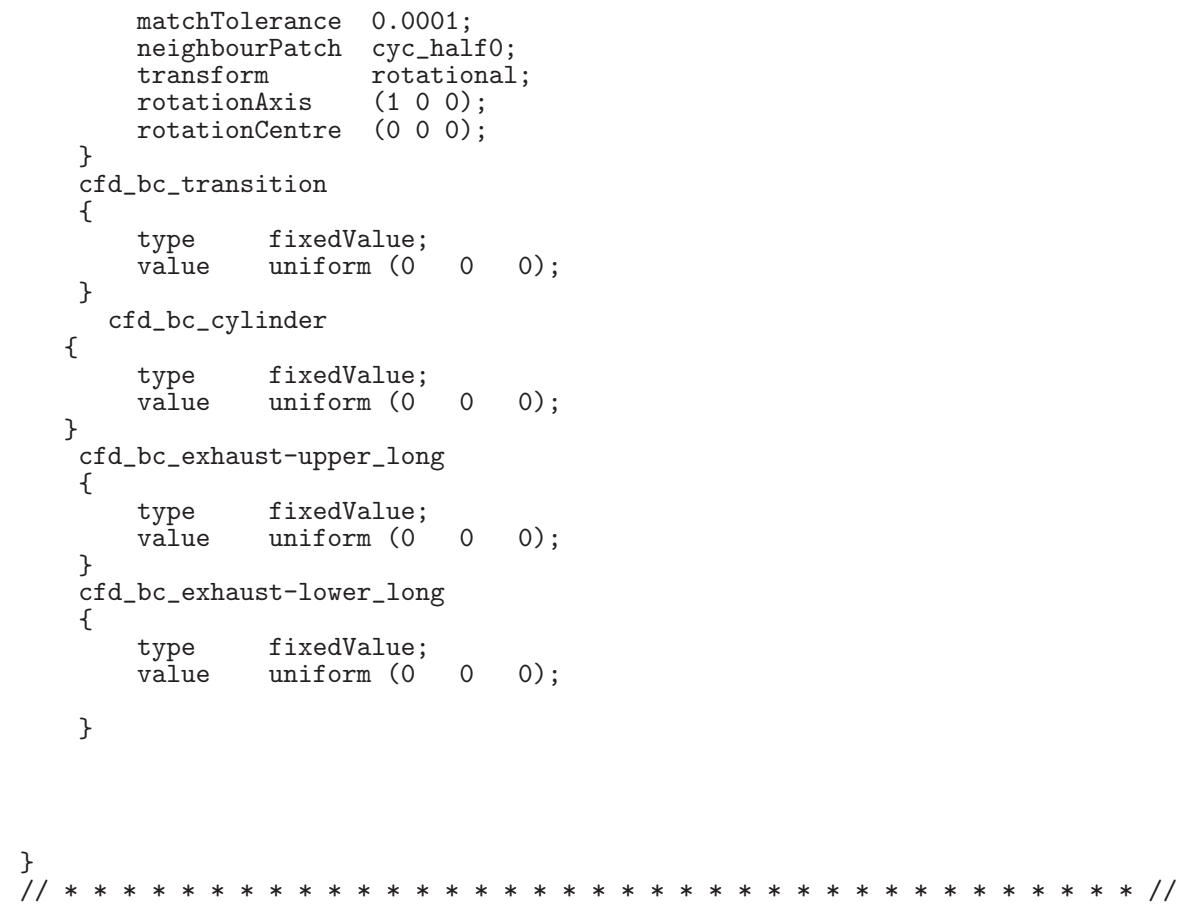

\section{A.2. Procedure how to generate cyclic patches}

cyclic patches generation

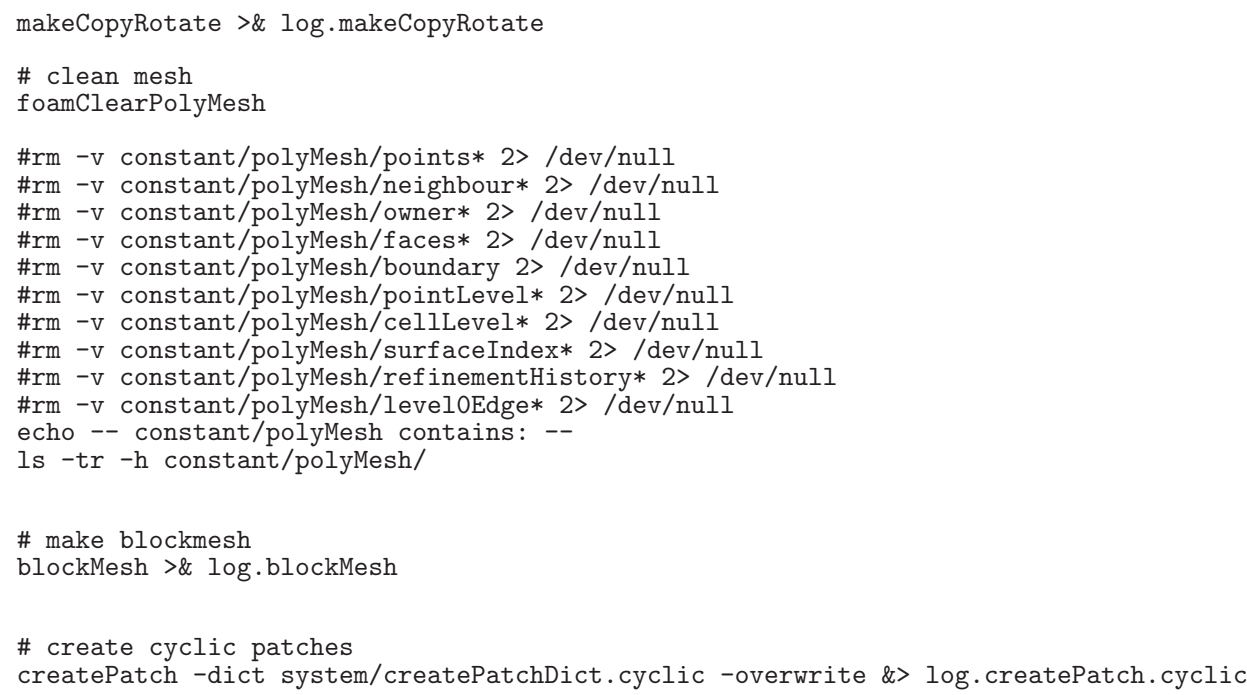


A. Appendix

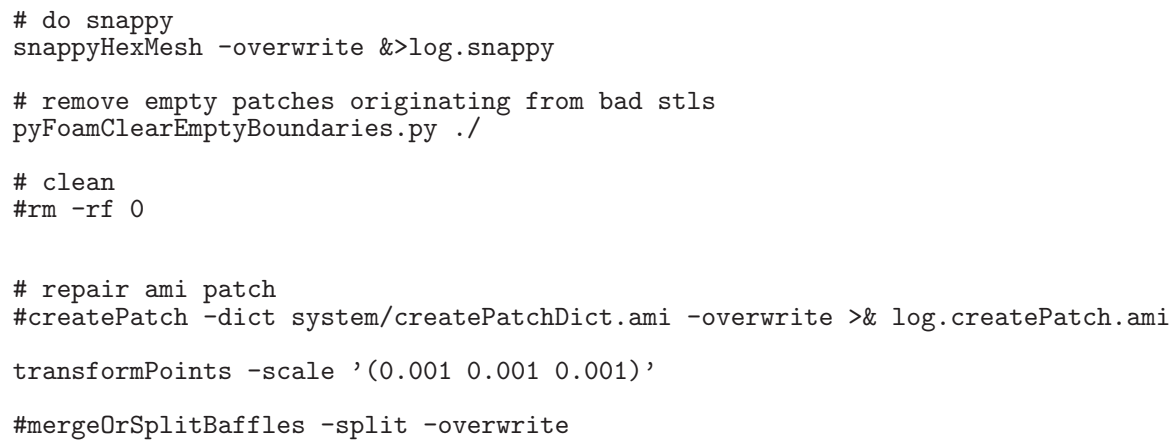

\section{A.3. Procedure how to build a 2D mesh with SnappyHexMesh}

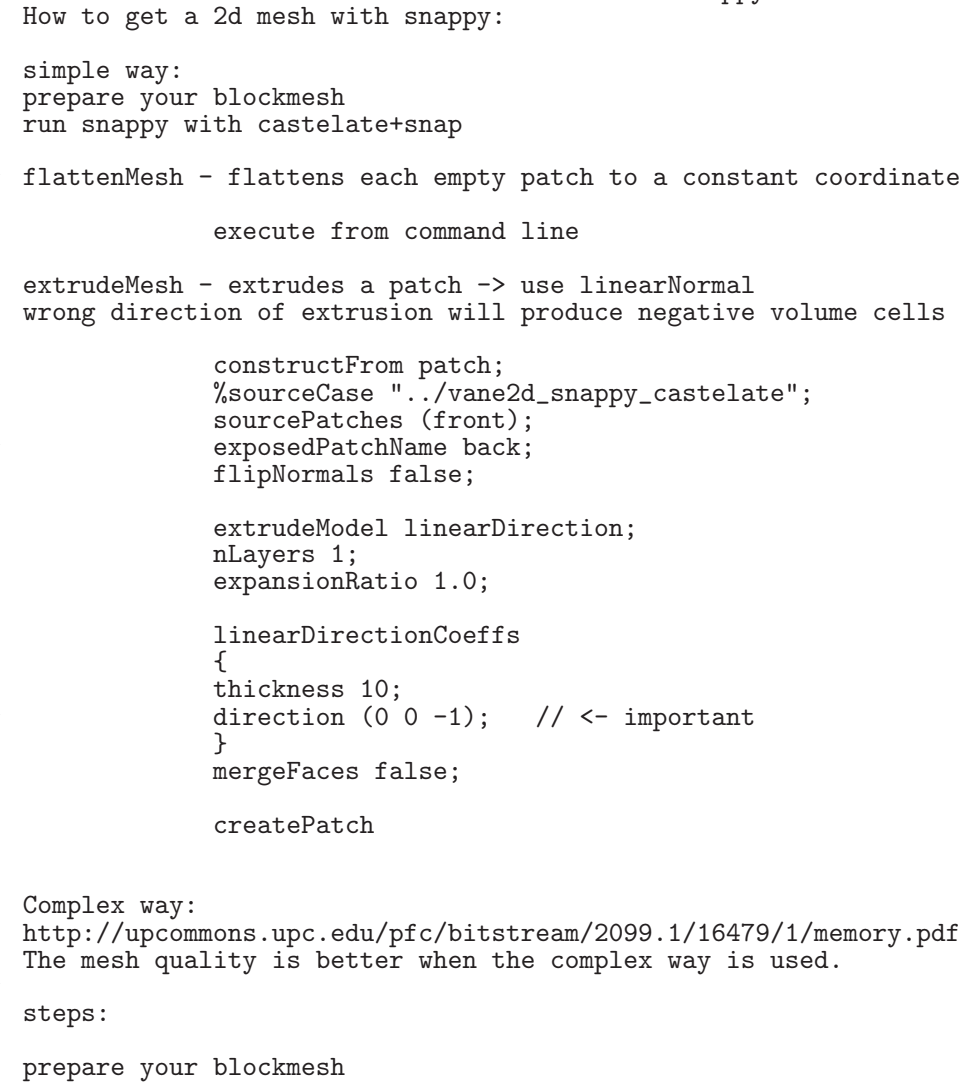




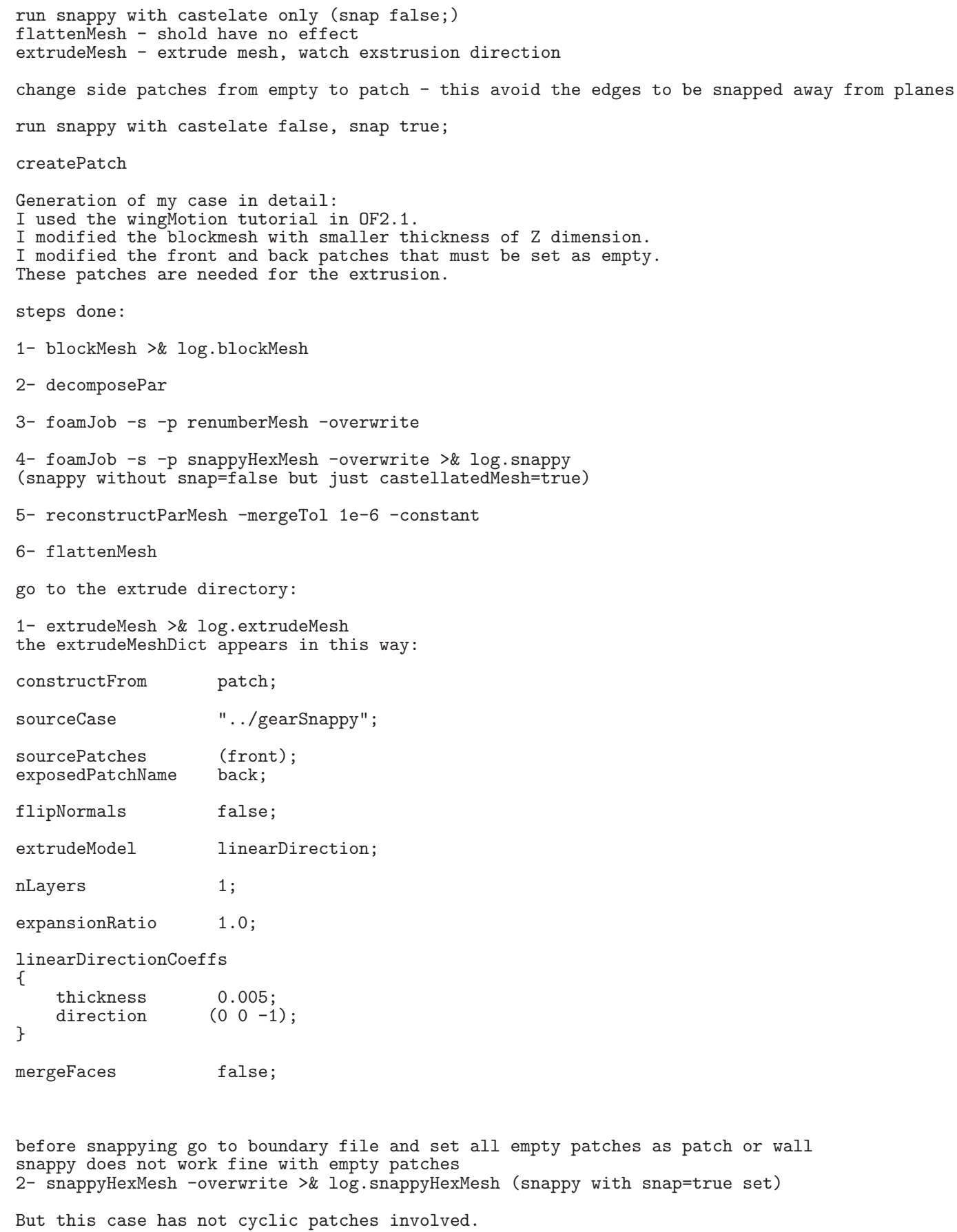


A. Appendix

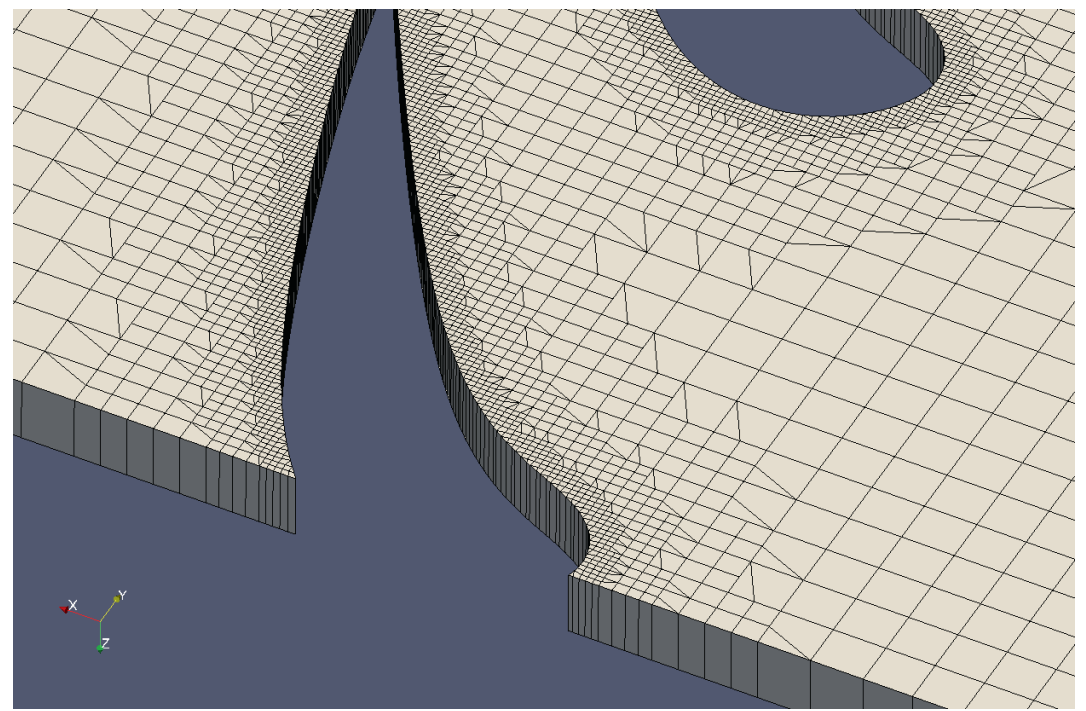

Figure A.1.: FlattenMesh of linearDirection extrusion of nonflat patch

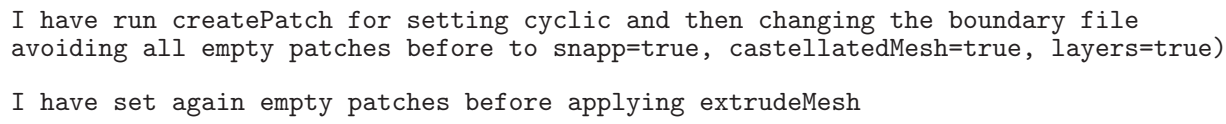

In Fig. A.1 and Fig. A.2 it has been shown how the tool FlattenMesh works. The application of the tool before the extrusion of the patch leads to a smooth mesh outline, as in Fig. A.1. A patch that has been extruded without the previous usage of the "flatten" command presents wiggles and sharp corners along all contour, see Fig. A.2.

\section{A.4. Study performed concerning rotating meshes}

Different tutorials related to the generation of moving-meshes with the old versions of OpENFOAM available only for incompressible flows are presented below.

\section{A.4.1. Periodic sector cylinder - Stator-Rotor-Stator application}

In Fig. A.3 the tool FunkySetBoundaryField has been used in order to set a swirl flow for the velocity field along the inlet patch. The solver RHOPimpleFoAm has been then applied.

\section{A.4.2. Cylinder rotating - OpenFOAM 1.6ext}

application= "icoDyMFoam" 
A.4. Study performed concerning rotating meshes

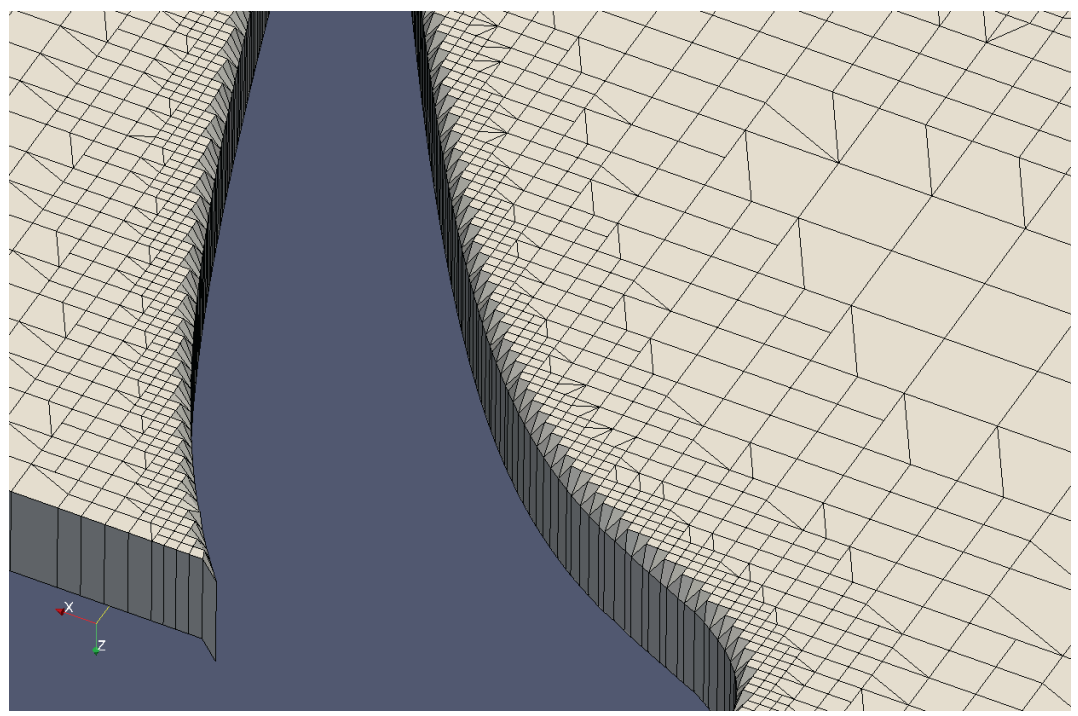

Figure A.2.: LinearDirection extrusion of nonflat patch

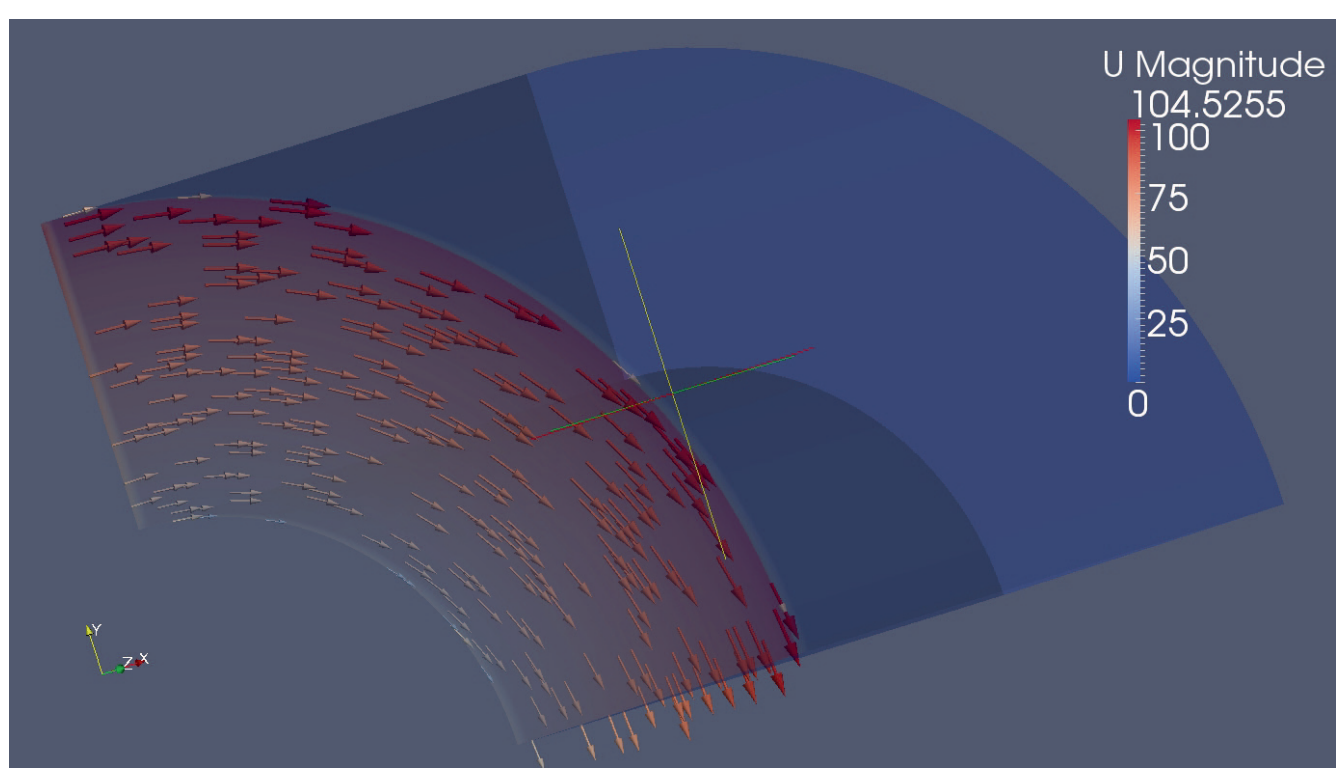

Figure A.3.: Inlet swirl detail 


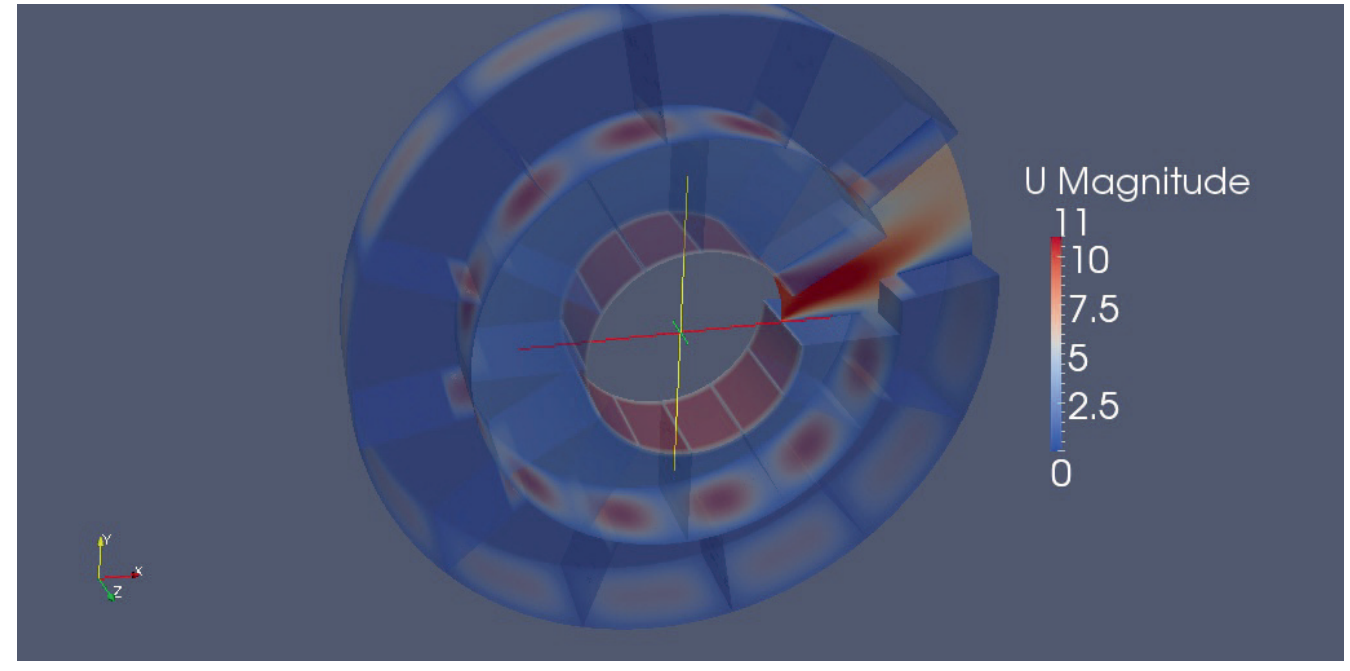

Figure A.4.: Turbo passage rotating overview

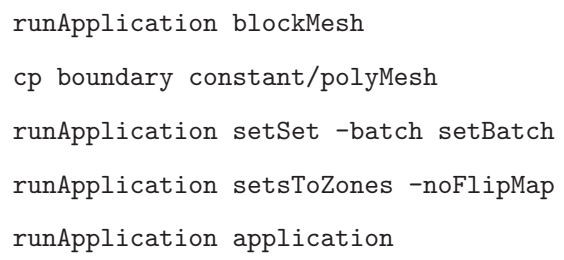

\section{A.4.3. Turbo Passage rotating - partial overlap GGI - OpenFOAM}

\section{$1.6 \mathrm{ext}$}

The numerical settings for cyclic and swirling boundary conditions with GGI (General Grid Interface in OPENFOAM 1.6 extended) and AMI (Arbitrary Mesh Interface in OpenFOAM 2.1) have been analyzed in detail. These settings have been investigated to obtain rotating meshes in order to evaluate the interactions between vanes and blades. An example of a rotating passage obtained by using GGI boundary conditions has been shown in Fig. A.4.

The procedure used to set a numerical simulation with moving mesh composed of statorrotor-stator sections is presented in detail and the resulting mesh can be observed in Fig. A.5. Moving Stator-Rotor-Stator - numerical procedure

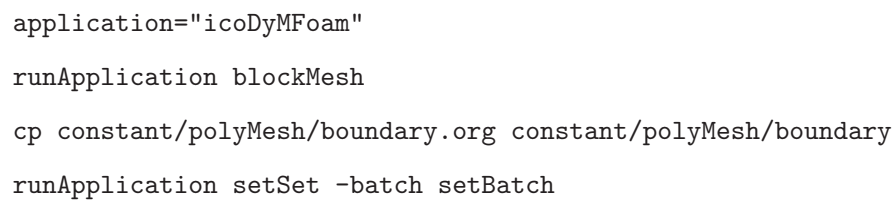




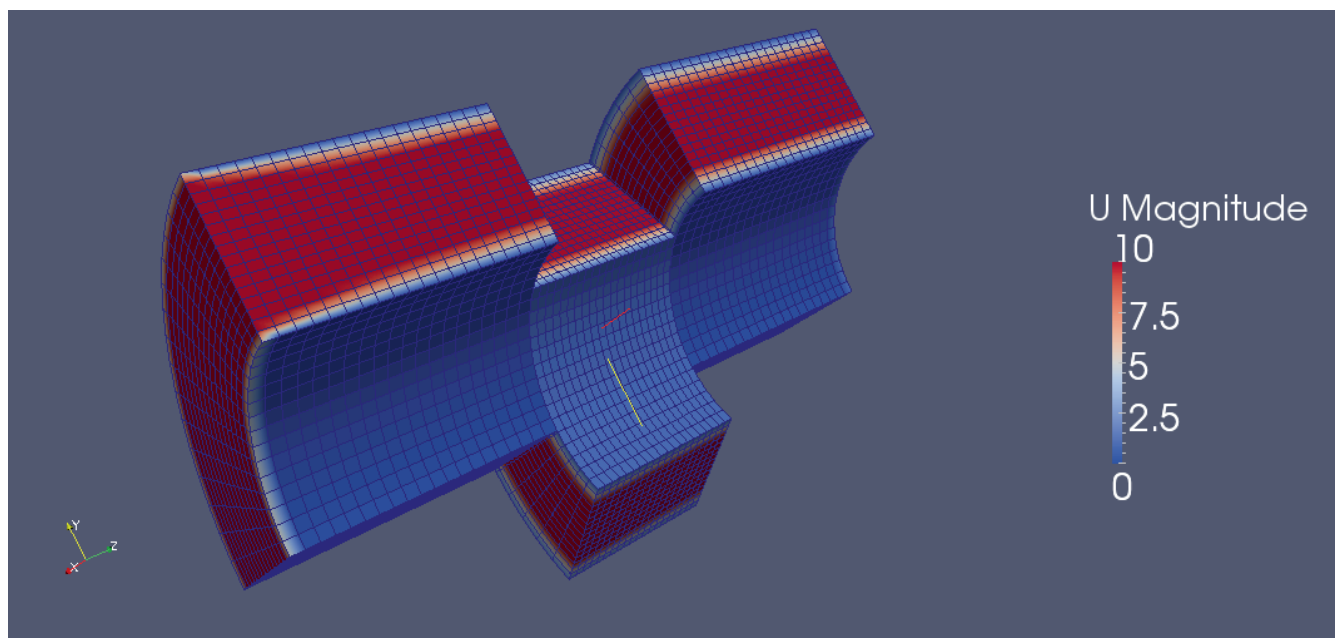

Figure A.5.: Moving Stator-Rotor-Stator

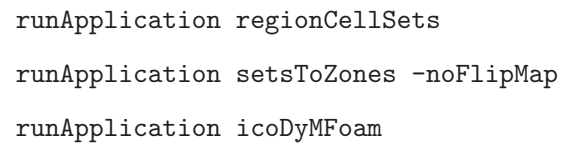

\section{A.4.4. Error compilation}

In this section a possible error that can occur during simulations with cyclic patches has been presented as example.

$$
\text { error compilation }
$$

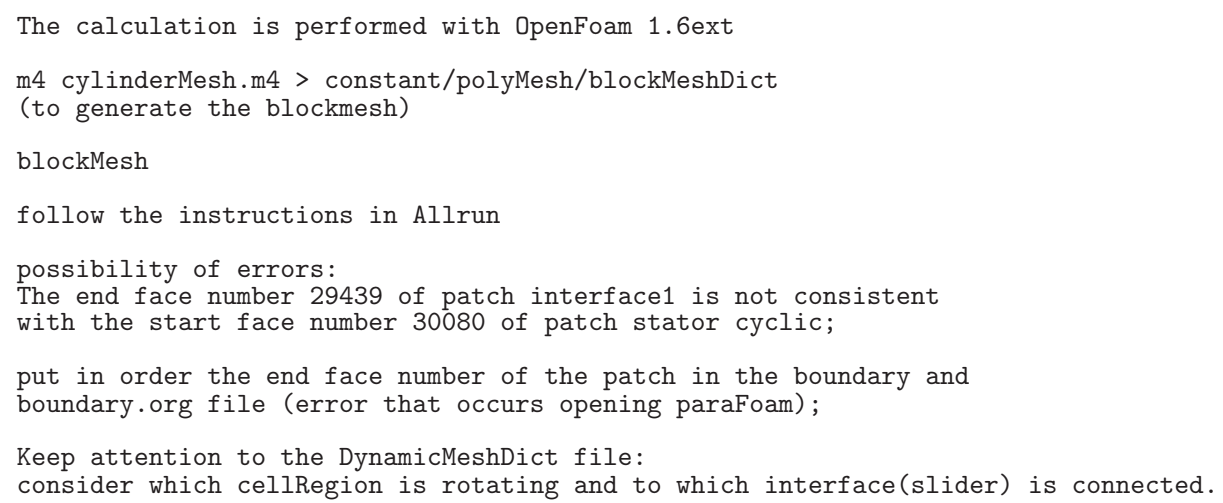




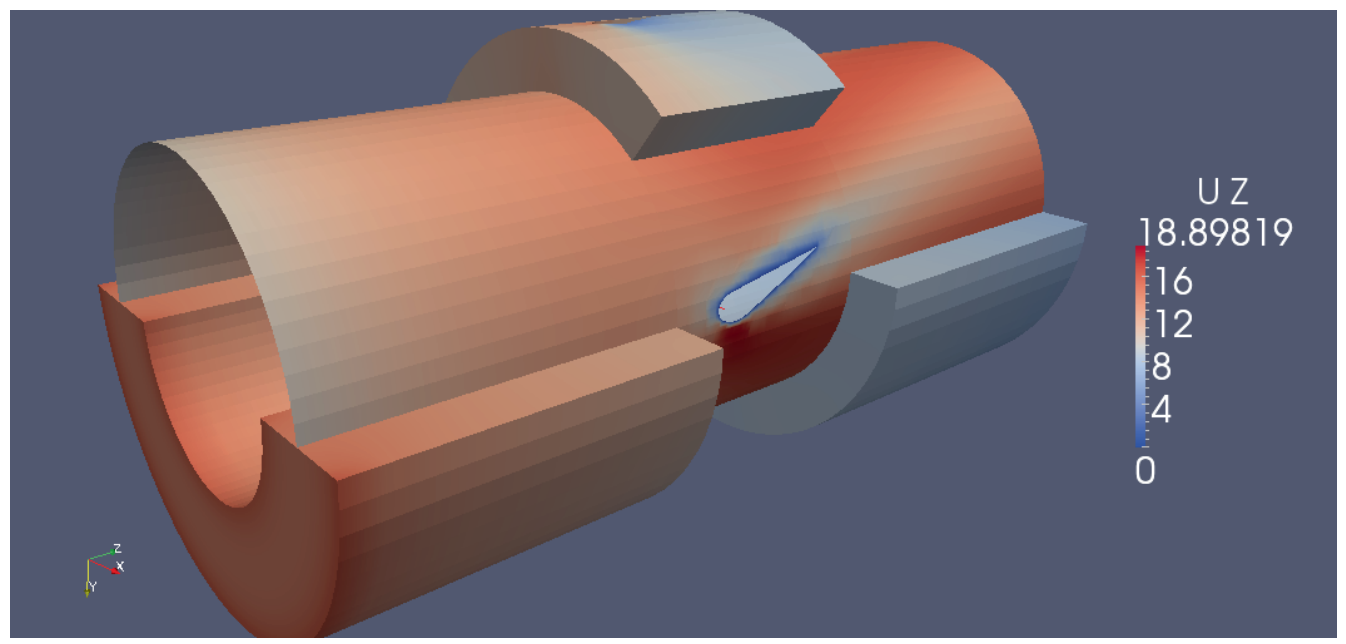

Figure A.6.: Cylinder Stator-Rotor-Stator

\section{A.4.5. Complex configurations}

The numerical procedure used to generate a complex moving mesh composed of stator-rotorstator sections is presented here and the outcome is shown in Fig. A.6.

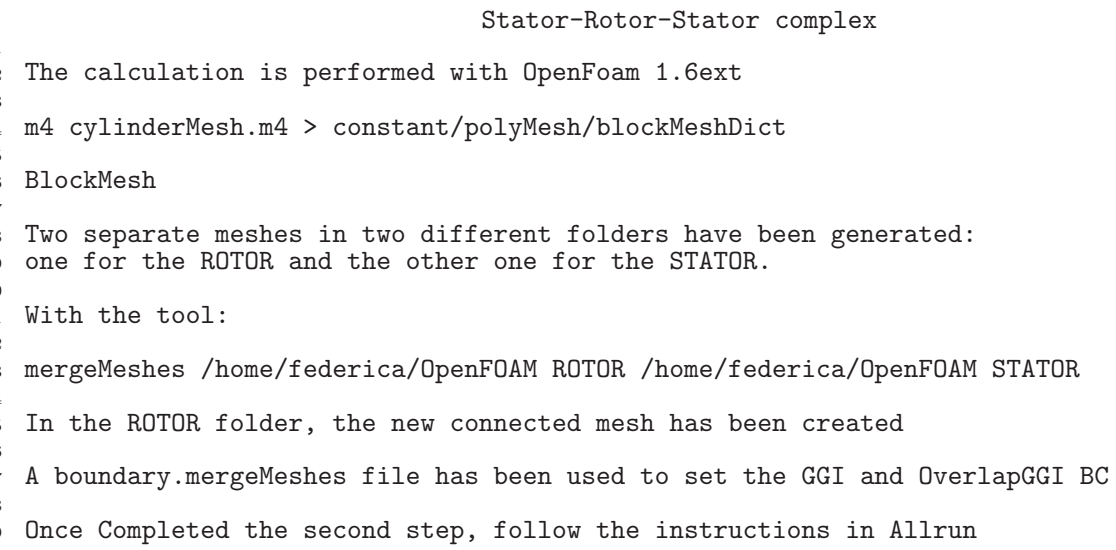

\section{A.5. 3D moving mesh Stator-Rotor generation}

As last, a moving-mesh combustion solver has been developed and Arbitrary Mesh Interfaces (AMI) have been extended to periodically repeating geometries with the scope to generate 3D moving meshes for compressible flows. Here the procedure used with the new solver implemented has been carried out. The cyclic AMI interfaces are poited out in Fig. A.7. The 
Fig. A.8 gives also a clear overview of the two AMI interfaces in case of a wedge BLOCKMESH configuration with one part rotating. This mesh has been generated in order to test the validity of the rotating interfaces.

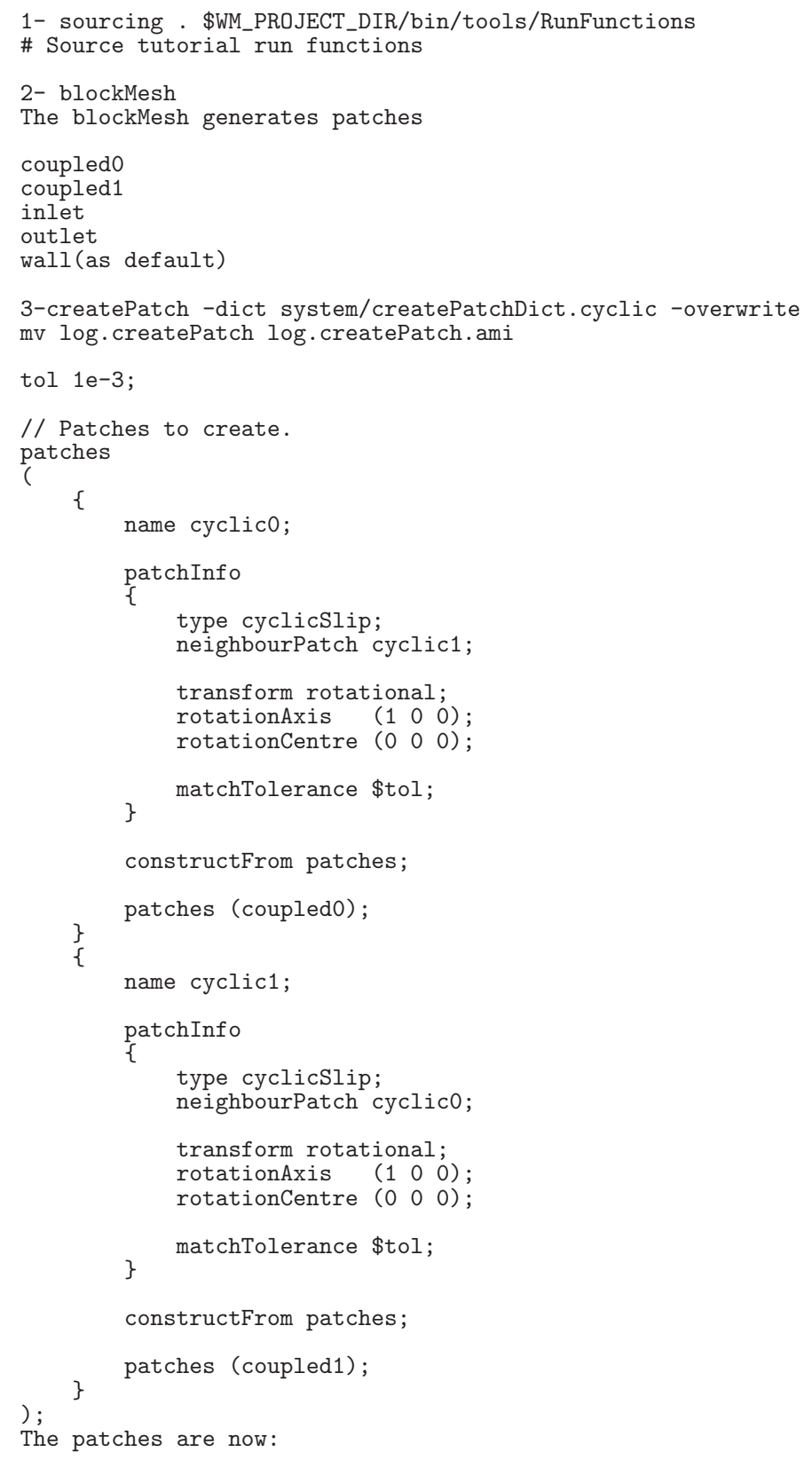




\section{A. Appendix}

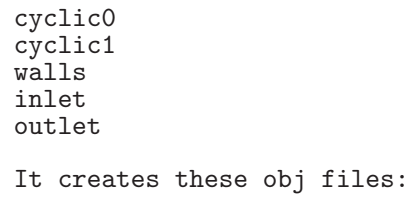

// If local number of cells is >= maxLocalCells on any processor

// switches from from refinement followed by balancing

// (current method) to (weighted) balancing before refinement.

maxLocalCells 100000;

// Overall cell limit (approximately). Refinement will stop immediately // upon reaching this number so a refinement level might not complete. // Note that this is the number of cells before removing the part which // is not 'visible' from the keepPoint. The final number of cells might 


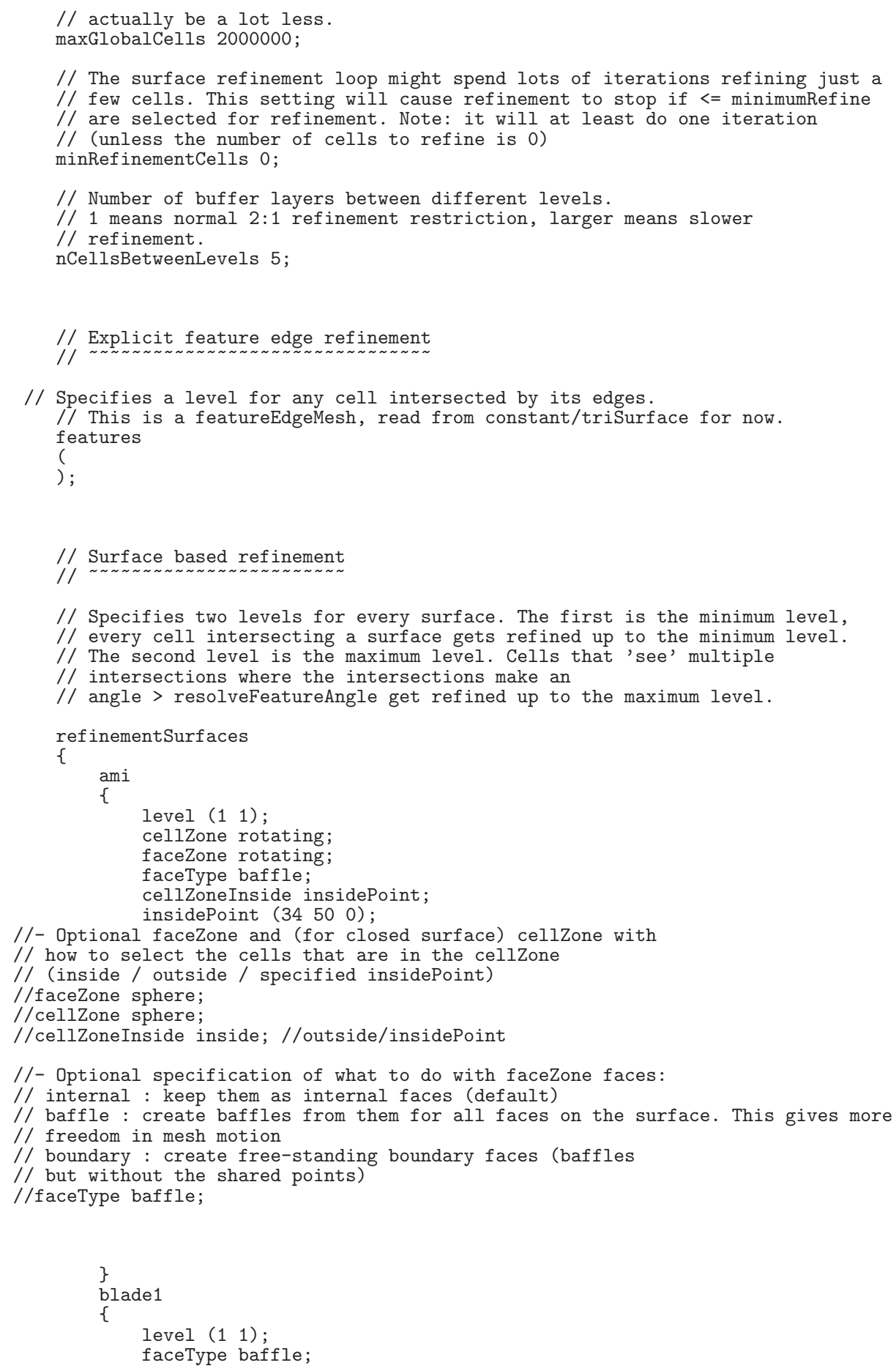


A. Appendix

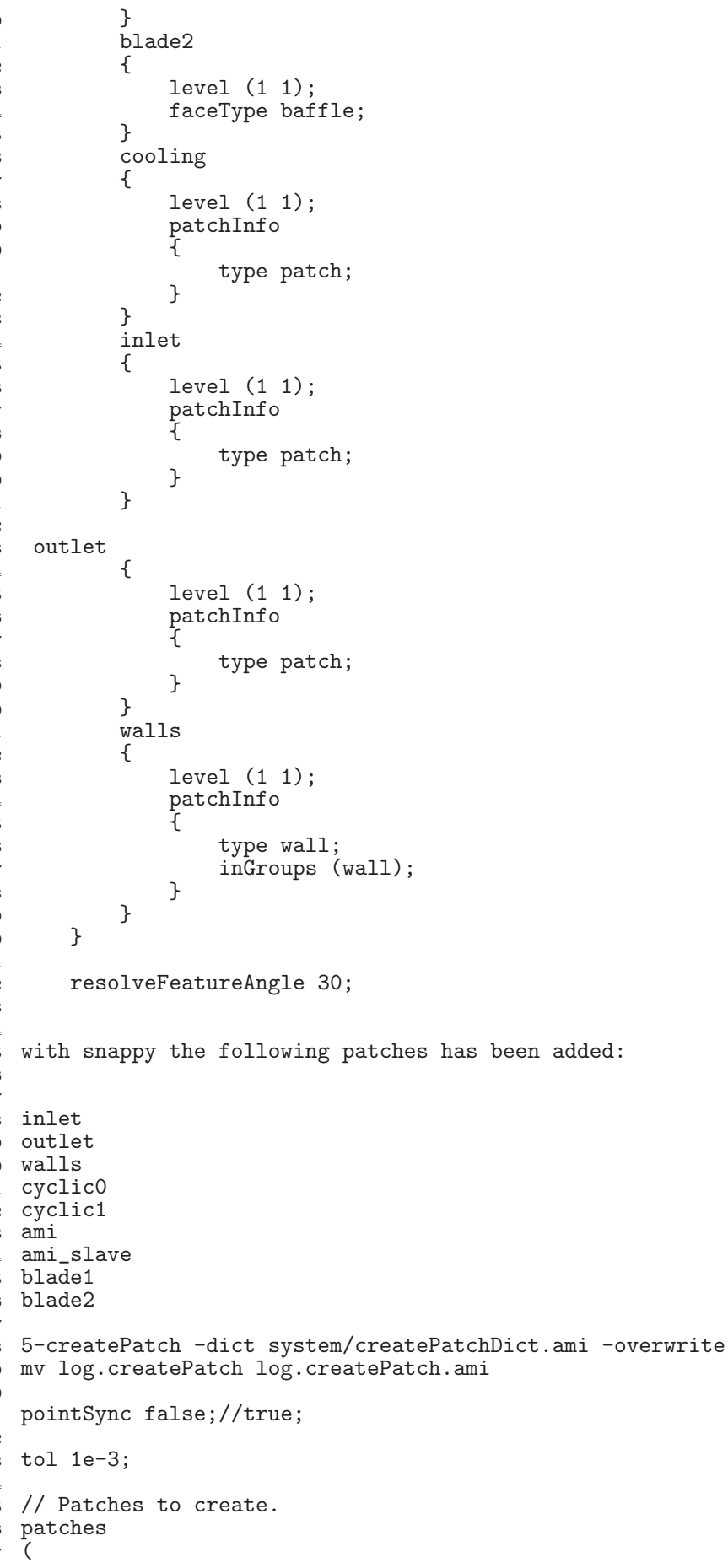




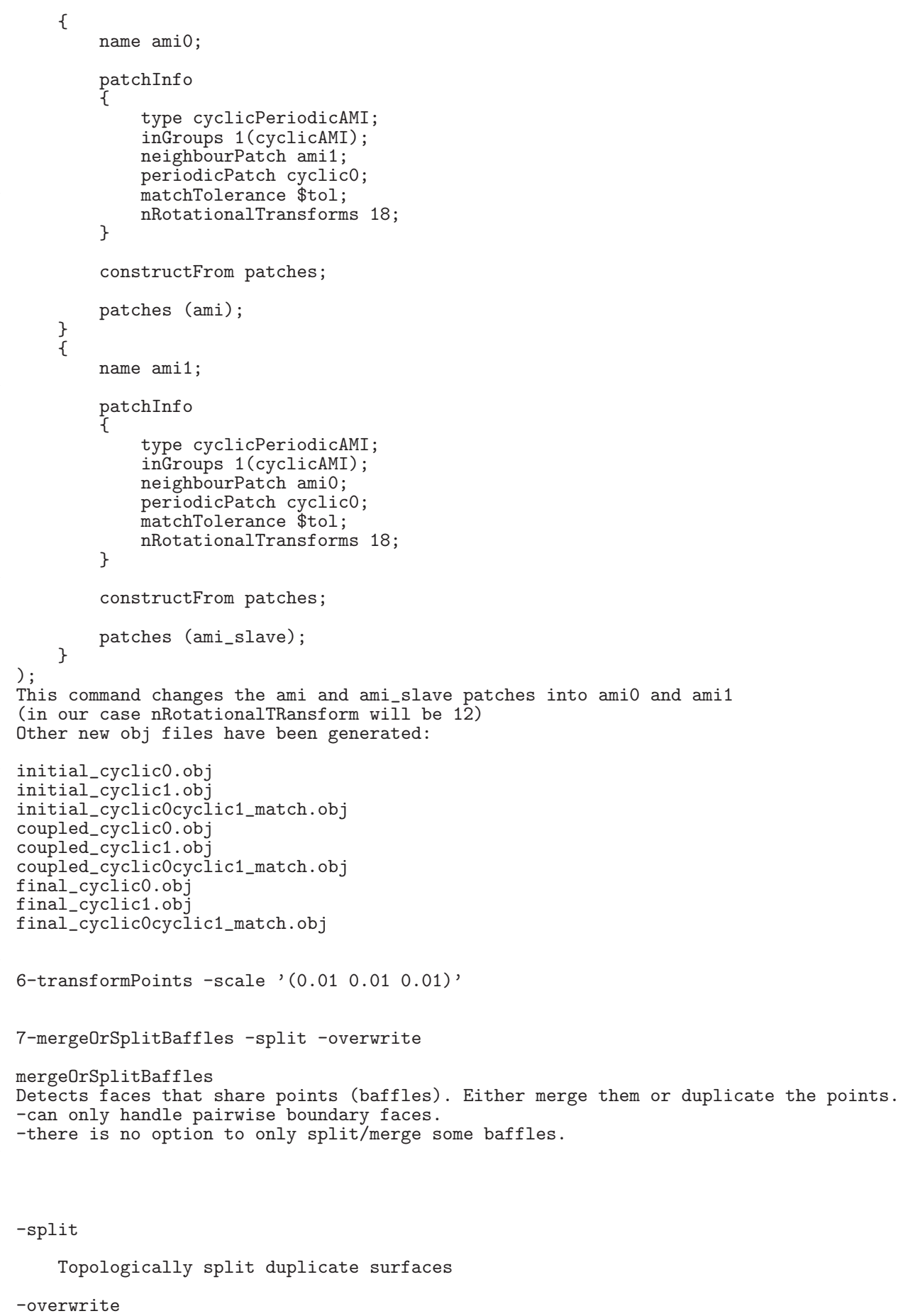




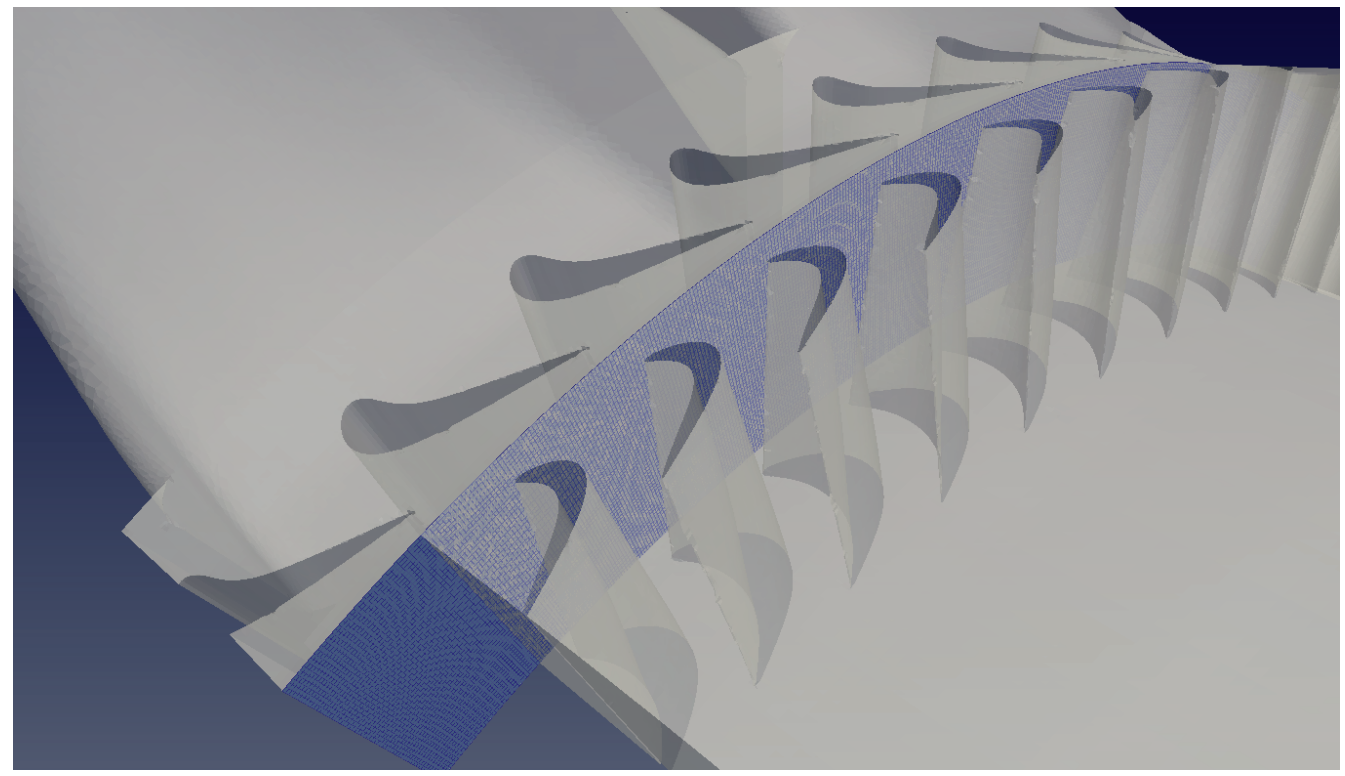

Figure A.7.: Cyclic AMI interfaces overview in the final mesh obtained

\section{A.6. Characterization of rotor effects on can-annular combustor acoustics}

\section{A.6.1. Introduction}

An extension of the current solver has been performed for the generation of moving meshes. A numerical simulation has been set up with the future purpose to realize a coupled study of all gas turbine components, evaluating the interaction between the various engine parts.

\section{A.6.2. Motivation for an extension of the current solver}

To extend the aforementioned studies from purely static geometries to the moving components as rotor blades, an extension of the current solver was necessary. The main reasons that led to the modification of the current solver are summarized below:

- In 2D and 3D: need of a periodic rotation and translation of a mesh zone coupled to the static part via a sliding interface 
A.6. Characterization of rotor effects on can-annular combustor acoustics

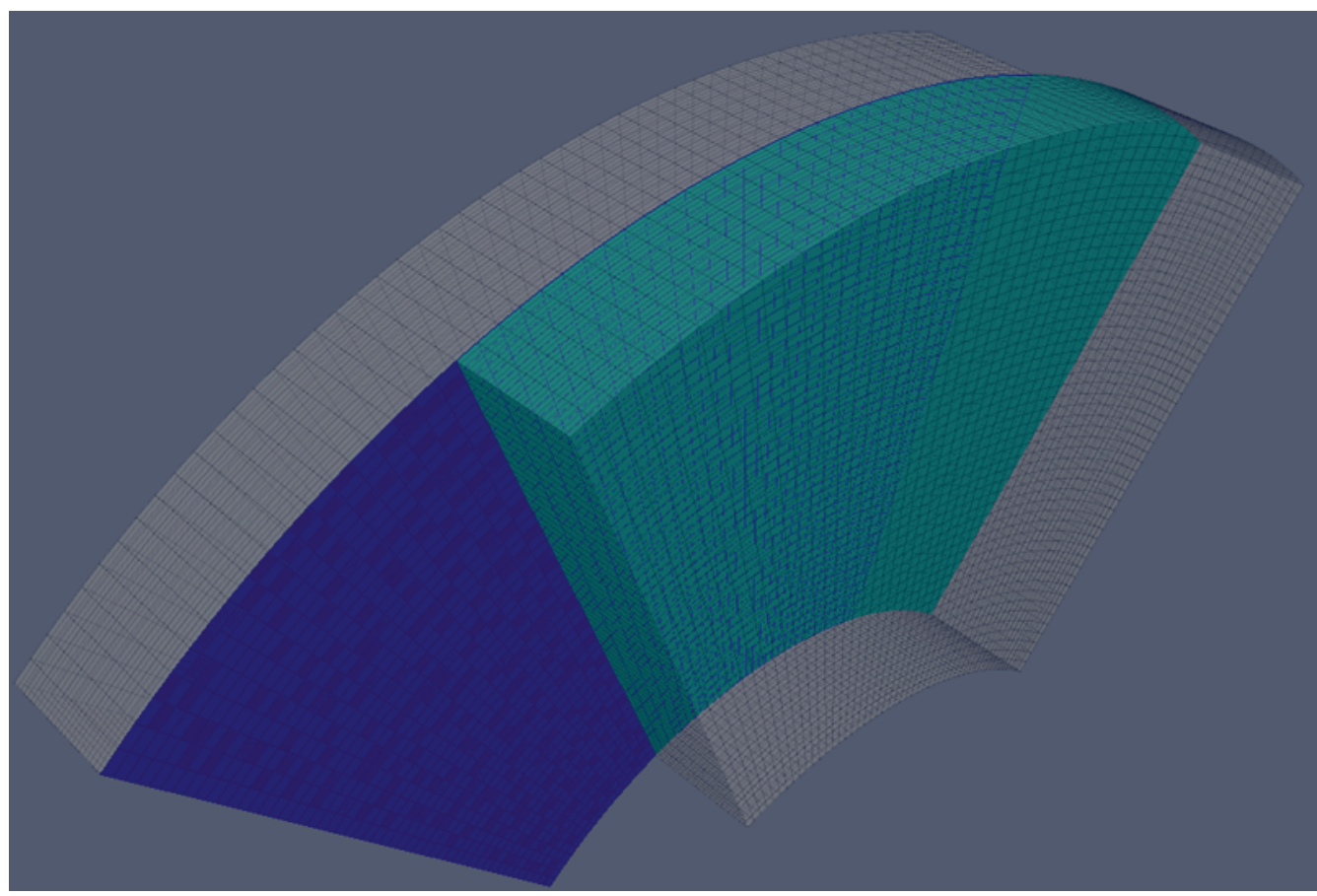

Figure A.8.: An example of cyclic AMI interfaces 
- Periodicity of boundary conditions in the moving mesh zone

- Equal sector angle in the static and moving mesh

- Reasonable conservancy and acoustic "neutrality" of sliding interface and periodic boundary conditions in moving part (second order method)

- Moving mesh capability added to the newest OPEnFOAM version (2.4) with possibility of working in parallel.

\section{A.6.3. Developed moving-mesh combustion solver and 3D mesh generation}

Moving meshes needed to be available to include rotor effects as last step for the research. For this purpose, a moving-mesh combustion solver has been developed and Arbitrary Mesh Interfaces (AMI) have been extended to periodically repeating geometries through the cooperation with ESI-OpenCFD. As next, the code has been compiled with the new interface feature implemented in the new OpenFOAM version (2.4). Before arriving to the mesh process, a problem occurred concerning the transition and the turbine geometry that were not matching. The cases were not aligned. The casing contours were not matching the transition in terms of the inner and outer radius as also the axial distance of the first vane and the transition lip. The conversion from IGES to STL files has been done in ICEM. The vanes and blades needed to be rotated. During the meshing process performed with SnAppyHexMesh, the static and rotating blades together with the AMI interfaces were included in the grid. The step then necessary to run AMI was to double the points at the interface done with MERGeOrSplitBaffles. The mesh generated in Fig. A.9 presented about 3 million of cells. The Fig. A.9 underlines the rotating mesh functionality applied to the turbine blade row. In the Appendix the procedure how to build a 3D moving-mesh with the developed solver is shown in detail. Different tutorials related to the generation of moving-meshes with the old solvers but with incompressible flow are also presented. A simulation has been run with single processor and in parallel. The test with parallel jobs failed and it needed debugging operations.

In order to test the rotating interface, a case has been estimated consisting of a wedge BLOCKMESH with one part rotating (see Fig. A.10).

No flow and walls at the inlet and outlet have been set, measuring the self-noise of the interface. Temperature and pressure remained constant and spurious wave did not appear, proving the validity of the rotating interface.

\section{A.6.4. Outlook}

A final simulation with the 3D generated mesh (see Fig. A.11) is now ongoing with the aim to post-process the acoustics of the case. The method applied to generate the final 3D mesh could be used as following to couple more different components of the whole gas turbine, leading to a more accurate analysis of the interaction between various engine parts. The 
A.6. Characterization of rotor effects on can-annular combustor acoustics

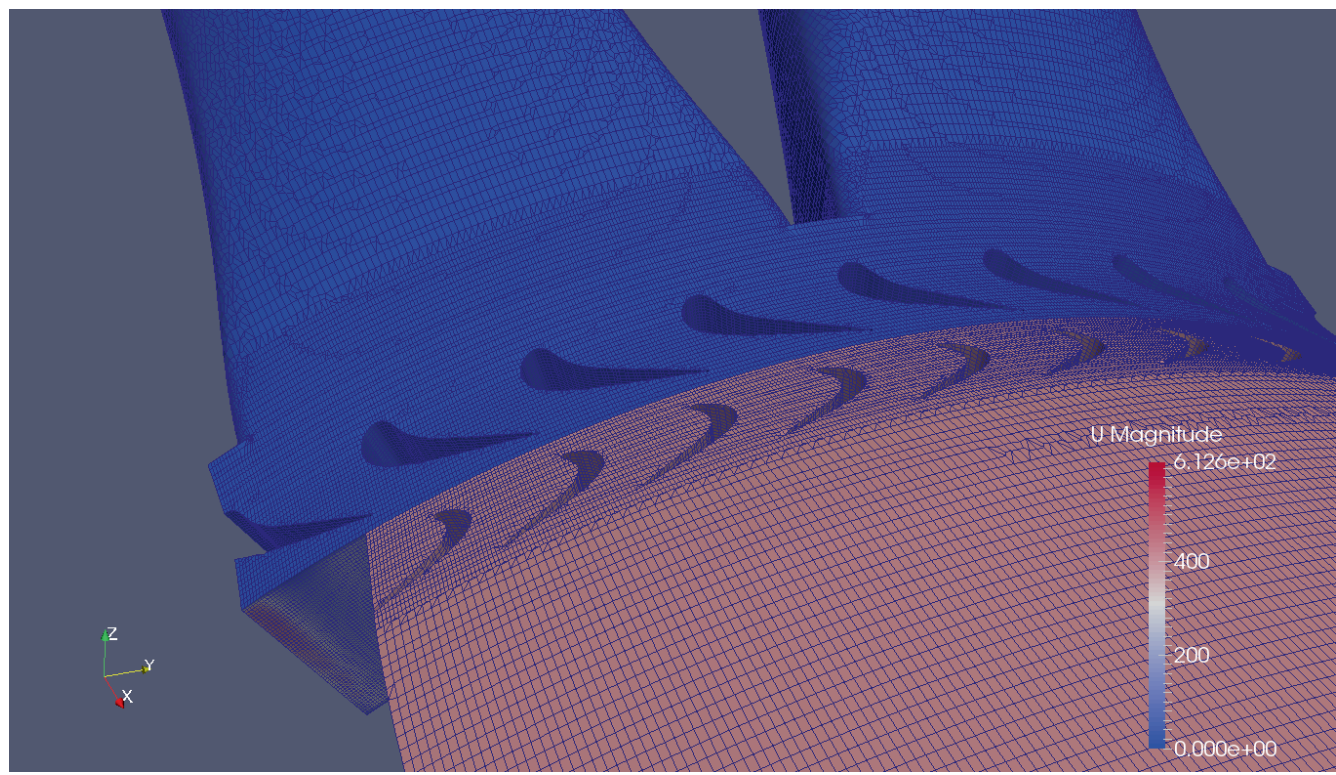

Figure A.9.: 3D mesh overview

Fig. A.12 shows an example of numerical simulation of a 3D configuration consisting of the whole circumferential set of cans coupled with the entire turbine vane stage. 
A. Appendix

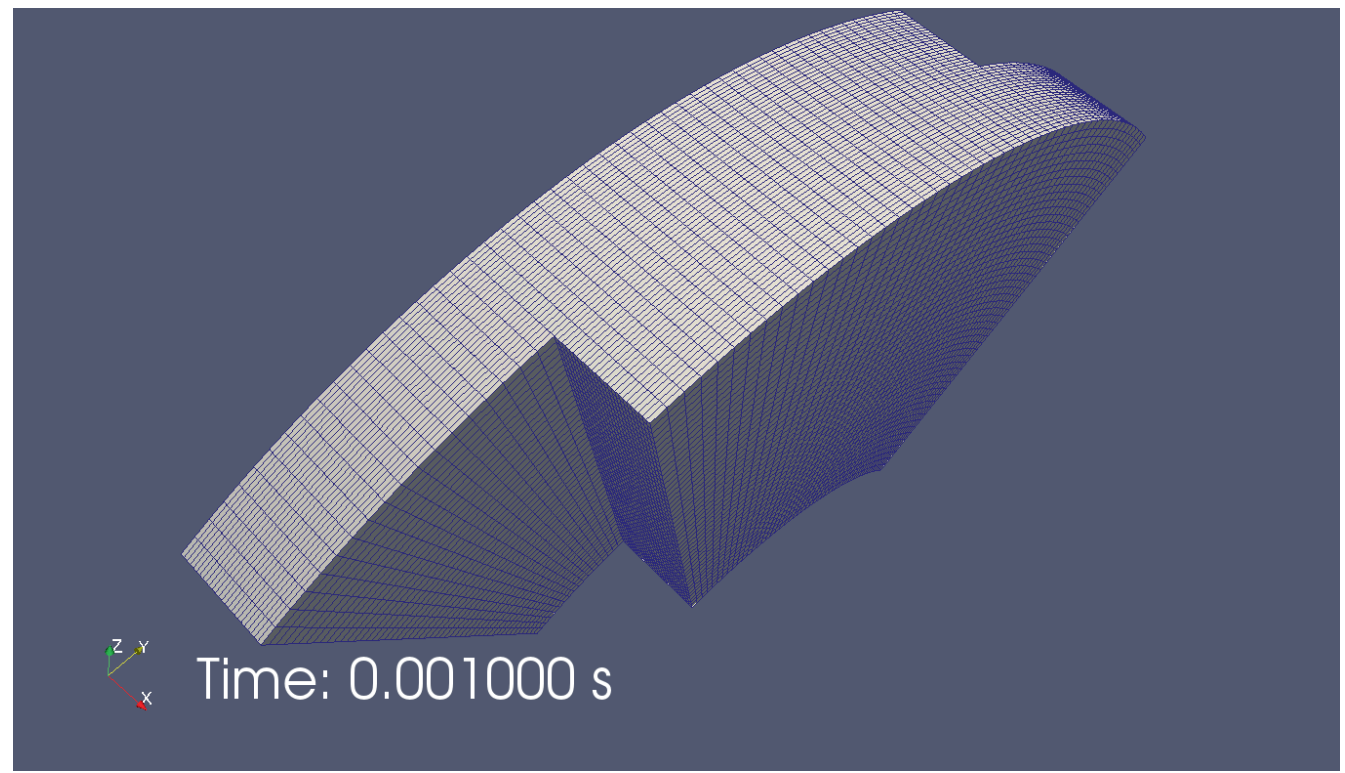

Figure A.10.: Wedge mesh overview

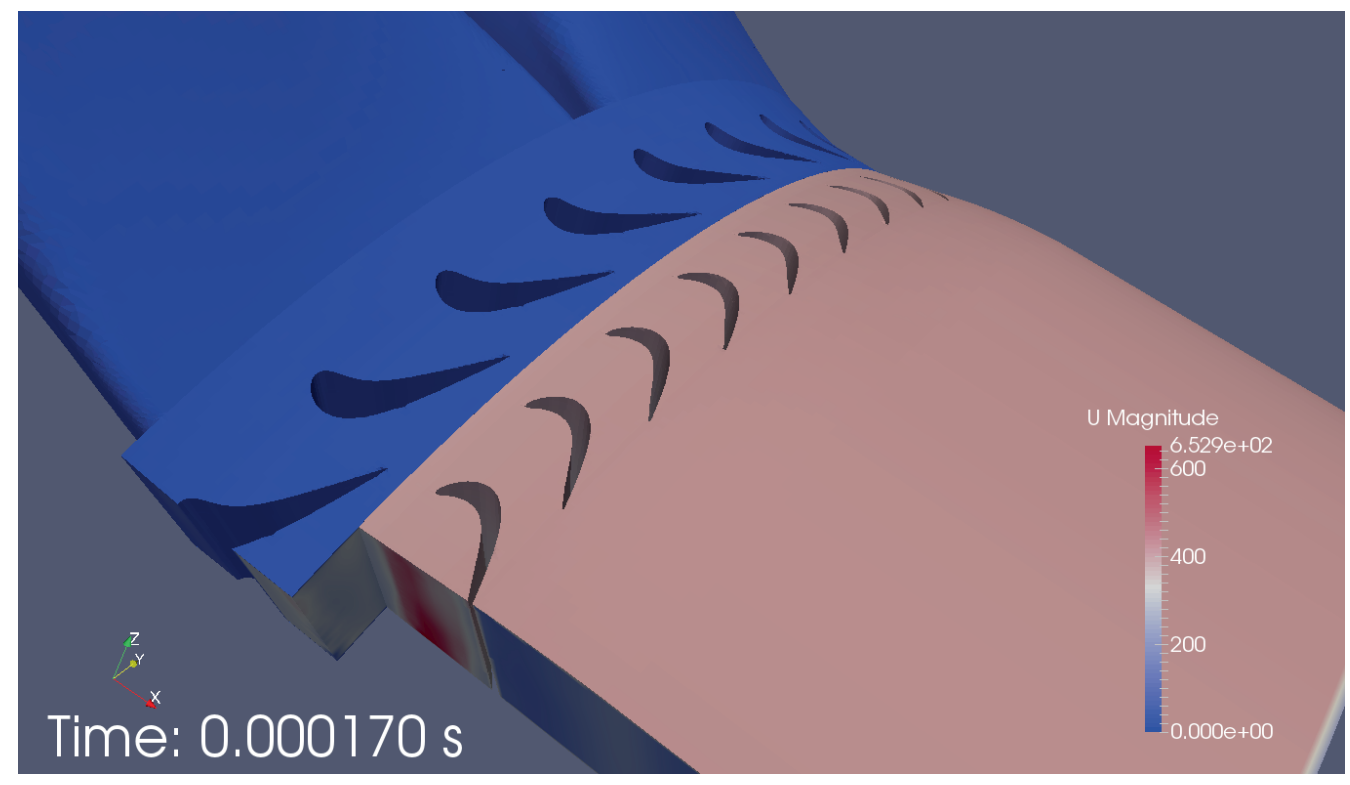

Figure A.11.: 3D generated moving mesh 
A.6. Characterization of rotor effects on can-annular combustor acoustics

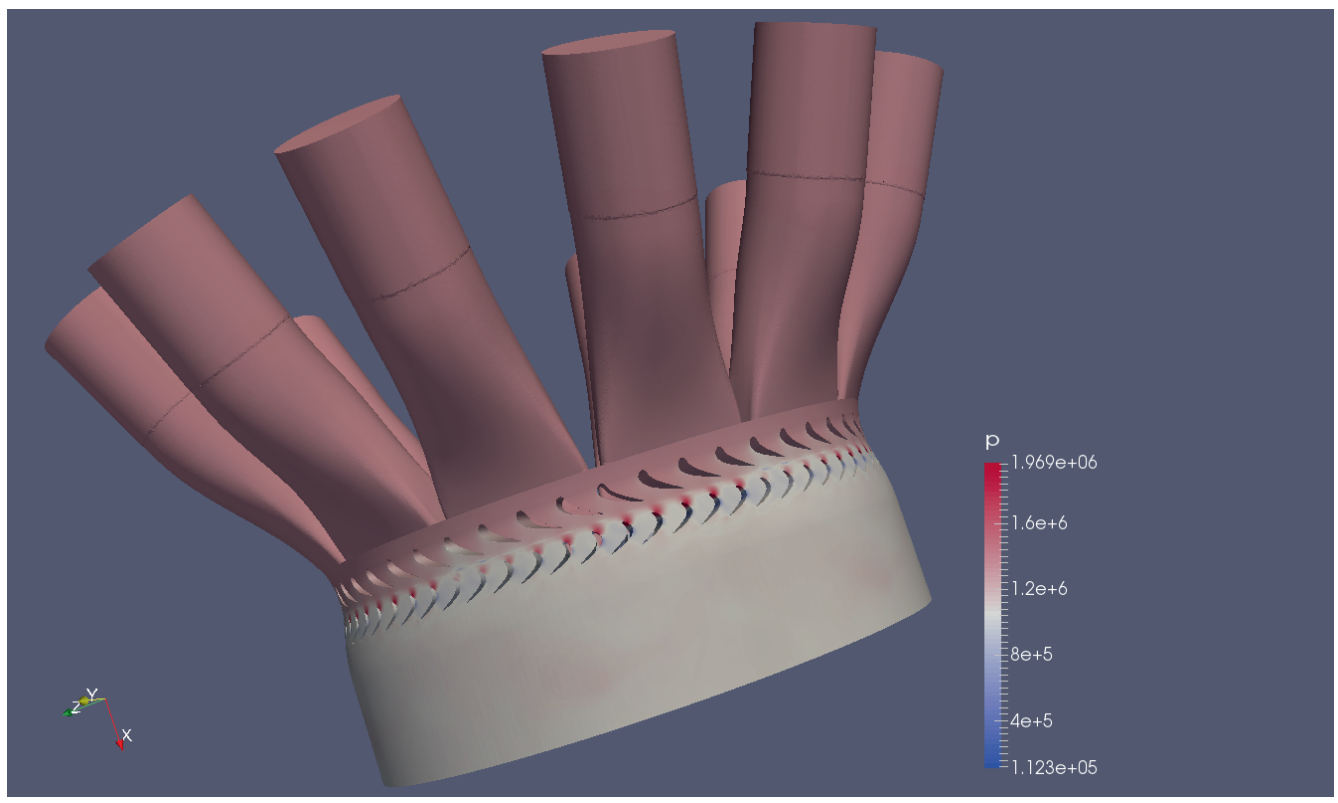

Figure A.12.: 3D generated final moving mesh 


\section{Bibliography}

[1] M. Abdulla Ghani, Simulation aux grandes echelles des instabilites de combustion transverses des flammes parfaitement premelangees et swirlees diphasiques, $\mathrm{PhD}$ Thesis, Universite' de Toulouse, 2015.

[2] Y. HuAng \& V. YAng, "Dynamics and stability of lean-premixed swirl-stabilized combustion," Prog. Energy Comb. Sci. 35(4), 293-364, 2009.

[3] C. LAHIRI, Acoustic performance of bias flow liners in gas turbine combustors, $\mathrm{PhD}$ Thesis, Technische Universität Berlin, 2014.

[4] C. BECK, "Validation report: Implementation of non reflecting boundary conditions in open- foam," Siemens internal report 2010.

[5] H. JASAK, "Turbo tools and general grid interface - theoretical basis and implementation," 6th OF Workshop 2011.

[6] N. Hanraths, Numerical Simulation of the Acoustic Response resulting from the Periodic Injection of Fluid into a Subsonic Nozzle, Master Thesis, Technische Universität Berlin, 2016.

[7] M. Munjal \& A. Doige, "The two-microphone method incorporating the effects of mean flow and acoustic damping," Journal of Sound and Vibration 137(1), 135-138, 1990 .

[8] M. Leyko, I. Duran, S. Moreau, F. Nicoud \& T. Poinsot, "Simulation and modelling of the waves transmission and generation in a stator blade row in a combustion-noise framework," Journal of Sound and Vibration 333, 6090-6106, 2014.

[9] R. Casado, Nonlinear Behavior of the Thermo Acoustic Instabilities in the Limousine Combustor, PhD Thesis, University of Twente, 2013.

[10] T. KuBIS, Analysis of the thermoacoustic behavior of gas turbine combustion systems based on large eddy simulations, Master Thesis, RWTH Aachen University, 2013.

[11] J. KELLER, "Thermoacoustic oscillations in combustion chambers of gas turbines," AIAA Journal 33(12), 2280-2287, 1995.

[12] T. Poinsot \& D. Veynante, Theoretical and Numerical Combustion, Second edition, Edwards, 2011, URL http://www.cerfacs.fr/elearning.

[13] F. Williams, Combustion Theory, Menlo Park, CA: Benjamin Cummings, 1985. 
[14] J. Rayleigh, The Explanation of certain Acoustical Phenomena, Nature 18, 319-321, 1878.

[15] T. LiEuWEn, Investigation of combustion instabilities mechanisms in premixed gas turbines, PhD Thesis, Georgia Institute of Technology, 1999.

[16] F. Nicoud \& T. Poinsot, "Thermoacoustic instabilities: Should the rayleigh criterion be extended to include entropy changes?" Combustion and Flame 142, 153-159, 2005.

[17] N. Cumpsty \& F. Marble, "The interaction of entropy fluctuations with turbine blade rows; a mechanism of turbojet engine noise," Proceedings of the Royal Society of London 1977.

[18] F. Marble, "Acoustic disturbance from gas nonuniformities through a nozzle," Proc. Interagency Symp. Univ. Res. Transp Noise 1973.

[19] F. Marble \& S. Candel, "Acoustic disturbances from gas nonuniformities convected through a nozzle," Journal of Sound and Vibration 55(2), 225-243, 1977.

[20] H. Tsien, "The transfer functions of rocket nozzles," Journal American Rocket Society 22, 139-143, 1952.

[21] L. Crocco, "Supercritical gaseous discharge with high frequency oscillations," Aerotechnica 33(1), 46-53, 1953.

[22] S. KaJi \& T. Okazaki, "Propagation of sound waves through a blade row: Analysis based on the acceleration potential method," Journal of Sound and Vibration 11, 355375, 1970.

[23] S. KaJi \& T. OkAzaki, "Propagation of sound waves through a blade row: I. analysis based on the semi-actuator disk theory," Journal of Sound and Vibration 11, 339-353, 1970.

[24] N. Lamarque \& T. Poinsot, "Boundary conditions for acoustic eigenmode computations in gas turbine combustion chambers," AIAA Journal 46(9), 2008.

[25] I. Duran, S. Moreau \& T. Poinsot, "Analytical and numerical study of direct and indirect combustion noise through a subsonic nozzle," AIAA Journal 51(1), 42-52, 2013.

[26] H. Posson \& M. Roger, "Parametric study of gust scattering and sound transmission through a blade row," 13th AIAA/CEAS Aeroacoustics Conference (28th AIAA Aeroacoustics Conference), Aeroacoustics Conferences 2007.

[27] H. Posson, S. Moreau, H. Bleriot, B. de l'Epine \& C. Schram, "Prediction of sound transmission through an annular cascade using an analytical cascade response function," 16th AIAA/CEAS Aeroacoustics Conference 2010.

[28] F. Farisco, L. PAneK \& J. KoK, "Thermo-acoustic coupling in can-annular combustors - a numerical investigation," ICSV22 International Congress on Sound and Vibration 2015. 
Bibliography

[29] A. Rasheed, A. Furman \& A. Dean, "Experimental investigations of an axial turbine driven by a multi-tube pulsed detonation combustor system," 41st AIAA/ASME/SAE/ASEE Joint Propulsion Conference and Exhibit, Joint Propulsion Conferences 2005.

[30] A. Rasheed, A. Furman \& A. Dean, "Wave attenuation and interactions in a pulsed detonation combustor-turbine hybrid system," 44th AIAA Aerospace Sciences Meeting and Exhibit 2006.

[31] M. Baptista, A. Rasheed, A. Badding, O. Velagandula \& A. Dean, "Mechanical response in a multi-tube pulsed detonation combustor-turbine hybrid system," 44th AIAA Aerospace Sciences Meeting and Exhibit 2006.

[32] N. Caldwell, A. Glaser \& E. Gutmark, "Experimental performance measurements of a pulse detonation engine integrated with an axial flow turbine," 42nd AIAA/ASME/SAE/ASEE Joint Propulsion Conference and Exhibit 2006.

[33] J. Deng, L. Zheng, C. Yan, L. Jiang, C. Xiong \& N. Li, "Experimental investigations of a pulse detonation combustor-turbine hybrid system," 47th AIAA Aerospace Sciences Meeting Including The New Horizons Forum and Aerospace Exposition 2009.

[34] F. Nicoud \& T. Poinsot, "Acoustic modes in combustors with complex impedances and multidimensional active flames," AIAA Journal 45(2), 2007.

[35] J. Blimbaum, M. Zanchetta, T. Akin, V. Acharya, J. O'Connor, D. Noble \& T. Lieuwen, "Transverse to longitudinal acoustic coupling processes in annular combustion chambers," International journal of spray and combustion dynamics 4(4), 275-298, 2012.

[36] N. Noiray \& B. Schuermans, "On the dynamic nature of azimuthal thermoacoustic modes in annular gas turbine combustion chambers," Proceedings of the Royal Society A 2013.

[37] M. Bauerheim \& T. Poinsot, "Analytical methods for azimuthal thermo-acoustic modes in annular combustion chambers," Center for Turbulence Research Annual Research Briefs 2015.

[38] L. Kinsler, A. Frey, A. Coppens \& J. Sanders, Fundamentals of Acoustics, John Wiley \& Sons, 4. Auflage, 2000.

[39] H. Schade, Strömungslehre, W. de Gruyter, Berlin, 1980.

[40] S. Rienstra \& A. Hirschberg, An Introduction to Acoustics, Eindhoven University

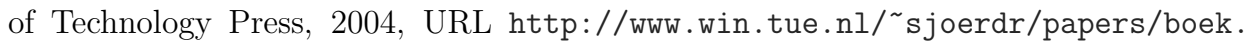
pdf.

[41] K. Ehrenfried, Strömungsakustik, Mensch \& Buch Verlag, Berlin, 2004.

[42] M. Shapiro, The dynamics and thermodynamics of compressible fluid flow, John Wiley \& Sons, Inc., 1953. 
[43] A. Munjal, Acoustics of Ducts and Mufflers, John Wiley \& Sons, Inc., 1987.

[44] G. N. Watson, A treatise on the theory of Bessel functions, Cambridge Univ. Press, Cambridge, 1922.

[45] J. Ferziger \& P. M., Computational Methods for Fluid Dynamics, Springer, Berlin, 3. Auflage, 2002.

[46] L. EulER, "Supplement aux recherches sur la propagation du son (supplement to the research on the propagation of sound)," Mémoires de l'académie des sciences de Berlin $15,210-240,1766$.

[47] A. Pierce, "Acoustics - an introduction to its physical principles and applications," Acoustical Society of America, New York 1989.

[48] G. Stokes, "On the theories of the internal friction of fluids in motion, and of the equilibrium and motion of elastic solids," Transactions of the Cambridge Philosophical Society 8, 287-319, 1845.

[49] G. Batchelor, An Introduction to fluid dynamics, Cambridge University Press, Cambridge, UK, 2000.

[50] R. Bird, W. Stewart \& E. Lightfoot, Transport Phenomena, 2nd ed., John Wiley \& Sons, 2002.

[51] B. Clapeyron, "Mémoire sur la puissance motrice de la chaleur," Journal de l'Ecole Royale Polytechique 14, 153-190, 1834.

[52] M. Goldstein, Aeroacoustics, McGraw-Hill, New York, 1976.

[53] J. Delfs, Basic of Aeroacoustics, lecture notes, Technische Universität Braunschweig, 2014.

[54] L. Euler, "Principes généraux du mouvement des fluides (general principles of the motion of fluids)," Mémoires de l'académie des sciences de Berlin 11, 274-315, 1757.

[55] D'Alembert, "Recherches sur la courbe que forme une corde tenduë mise en vibration (investigation of the curve formed by a vibrating string)," Histoire de l'Académie des sciences et belles-lettres 3, 214-219, 1747.

[56] L. Euler, "De la propagation du son (on the propagation of sound)," Mémoires de l'académie des sciences de Berlin 15, 185-209, 1766.

[57] I. Bronshtein, K. Semendyayev, G. Musiol \& H. Mülig, Handbook of Mathematics, 5th ed., Springer, Berlin, 2007.

[58] F. Holste, Ermittlung der aerodynamische Lärmquellen und Berechnung des abgestrahlten Schall mittels der im Nahfeld gemessenen Druckschwankungen am Beispiel eines Triebwerksmodells, PhD Thesis, Techische Universität Berlin, 1995. 
Bibliography

[59] B. Stahl, "Experimentelle untersuchung zur schallerzeugung durch die turbulenz in einer rohrstroemung hinter einer unstetigen querschnittserweiterung," Acta Acoustica United with Acoustica 63(1), 42-59, 1987.

[60] W. Neise \& U. Michel, "Aerodynamic noise of turbomachines," Report DLR-IB22314-94/B5, DLR 1994.

[61] M. Duhamel, "Sur les vvibration des gaz dans des tuyaux cylindriques, coniques, etc." Journal de mathématiques pures et appliquées 14, 49-110, 1849.

[62] J. Rayleigh, "On waves," Philosophical Magazine Series 5 1(4), 257-279, 1876.

[63] J. Tyler \& T. Sofrin, "Axial flow compressor noise studies," SAE Transaction 70, 309-332, 1962.

[64] V. MAson, "Some experiments on the propagation of sound along a cylindrical duct containing flowing air," Journal of Sound and Vibration 10(2), 208-226, 1969.

[65] A. Michalke, "On the propagation of sound generated in a pipe of circular crosssection with uniform mean flow," Journal of Sound and Vibration 134(2), 203-234, 1989 .

[66] C. Morfey, "Sound transmission and generation in ducts with flow," Journal of Sound and Vibration 14(1), 37-55, 1971.

[67] M. Abramowitz \& I. Stegun, Handbook of mathematical functions, Applied Mathematics Series 55, National Bureau of Standards, 1970.

[68] J. Fourier, Théorie analytique de la chaleur (The analytic theory of heat), F. Didot, Paris, 1822.

[69] L. BeraneK, "Acoustics," Acoustical Society of America 1993.

[70] P. Morse, Vibration and sound, 2nd ed., McGraw-Hill, New York, 1948.

[71] A. Dowling, "The calculation of thermoacoustic oscillations," Journal of Sound and Vibration 180(4), 557-581, 1995.

[72] B. Mugridge, "Combustion driven oscillations," Journal of Sound and Vibration 70, 437-452, 1980.

[73] J. Bourgouin, D. Durox, T. Schuller, J. Beaunier \& S. Candel, "Ignition dynamics of an annular combustor equipped with multiple swirling injectors," Combust. Flame 160(8), 1398-1413, 2013.

[74] G. Staffelbach, L. Gicquel, G. Boudier \& T. Poinsot, "Large eddy simulation of self excited azimuthal modes in annular combustors," Proc. Combust. Inst. 32(2), 2909-2916, 2009.

[75] N. Worth \& J. Dawson, "Self-excited circumferential instabilities in a model annular gas turbine combustor: Global flame dynamics," Proc. Combust. Inst. 34, 1-8, 2012. 
[76] F. Richecoeur, F. Scouflaire, S. Ducruix \& S. Candel, "High-frequency transverse acoustic coupling in a multiple-injector cryogenic combustor," J. Prop. Power 22(4), 790-799, 2006.

[77] L. Selle, G. Lartigue, T. Poinsot, R. Koch, K. Schildmacher, W. Krebs, B. Prade, P. Kaufmann \& D. Veynante, "Compressible large-eddy simulation of turbulent combustion in complex geometry on unstructured meshes," Combust. Flame 137(4), 489-505, 2004.

[78] J. Schwing \& T. Sattelmayer, "Highfrequency instabilities in cylindrical flame tubes: Feedback mechanism and damping," ASME Turbo Expo 2013: Turbine Technical Conference and Exposition 2013.

[79] M. Zellhuber, J. Schwing, B. Schuermans, T. Sattelmayer \& W. Polifke, "Experimental and numerical investigation of thermoacoustic sources related to highfrequency instabilities," International Journal of Spray and Combustion Dynamics 6(1), $1-34,2014$.

[80] I. Hernandez, B. Cuenot \& T. Poinsot, "Les and acoustic analysis of thermoacoustic instabilities in a partially premixed model combustor," INCA Conference, Toulouse 2011.

[81] P. Hield, M. Brear \& S. Jin, "Thermo-acoustic limit cycles in a premixed laboratory combustor with open and choked exits," Combustion and Flame 156, 1683-1697, 2009.

[82] K. Kostrzewa, Advanced computational methods in identification of thermo-acoustic systems, PhD Thesis, University of Stuttgart, 2009.

[83] "Openfoam," URL https://github.com/OpenFOAM/OpenFOAM-2.1.x/blob/master/ src/turbulenceModels/compressible/LES/Smagorinsky/Smagorinsky.H.

[84] "Openfoam," URL https://github.com/OpenFOAM/OpenFOAM-2.1.x/blob/master/ src/turbulenceModels/compressible/LES/oneEqEddy/oneEqEddy.H.

[85] H. Weller, G. Tabor, H. Jasak \& C. Fureby, "A tensorial approach to computational continuum mechanics using object-oriented techniques," Computers in physics 12(6), 620-631, 1998.

[86] C. Greenshields, OpenFOAM User Guide, OpenFOAM Foundation Ltd, 2015, URL http://foam. sourceforge.net/docs/Guides-a4/UserGuide.pdf.

[87] C. Greenshields, OpenFOAM Programmer's Guide, OpenFOAM Foundation Ltd, 2015, URL http://foam.sourceforge.net/docs/Guides-a4/ProgrammersGuide. pdf.

[88] T. Holzmann, Mathematics, Numerics, Derivations and OpenFOAM, 2016, URL Tobias.HolzmannHolzmann-cfd.de.

[89] R. IssA, "Solution of the implicitly discretised fluid flow equations by operatorsplitting," Journal of Computational Physics 62, 40-65, 1986. 
[90] H. JASAK, Error analysis and estimation in the Finite Volume method with applications to fluid flows, PhD Thesis, Imperial College, University of London, 1996.

[91] S. Yuen, A. Gentemann \& W. Polifke, "Investigation of the influence of boundary conditions on system identifiability using real time system modeling," ICSV11 International Congress on Sound and Vibration 2004.

[92] T. Poinsot \& S. Lele, "Boundary conditions for direct simulations of compressible viscous flows," Journal of Computational Physics 101, 104-129, 1992.

[93] R. Kaess, A. Huber \& W. Polifke, "A time-domain impedance boundary condition for compressible turbulent flow," 14th AIAA/CEAS Aeroacoustics Conference (29th AIAA Aeroacoustics Conference), Aeroacoustics Conferences 2008.

[94] M. Beaudoin \& H. JASAK, "Development of a generalized grid interface for turbomachinery simulations with openfoam," OpenSource CFD International Conference 2008 .

[95] A. Seybert \& D. Ross, "Experimental determination of acoustic properties using a two-microphone random-excitation technique," Journal Acoust. Soc. Am. 61(5), 1977.

[96] F. Farisco, L. Panek, B. Janus \& J. Kok, "Numerical investigation of the thermoacoustic influence of the turbine on the combustor," ASME Turbo Expo, Montreal 2015.

[97] M. Bothien, J. Moeck \& C. PAschereit, "Active control of the acoustic boundary conditions of combustion test rigs," Journal of Sound and Vibration 318, 678-701, 2008.

[98] F. YAnG, Z. Guo, X. Fu \& D. YU, "Computation of acoustic transfer matrices of swirl burner with finite element and acoustic network method," Journal of Low Frequency Noise, Vibration and Active Control 2015.

[99] W. Polifke \& C. WALl, "Non-reflecting boundary conditions for acoustic transfer matrix estimation for les," Center for Turbulence Research Proceedings of the Summer Program 2002.

[100] W. Polifke, C. Wall \& P. Moin, "Partially reflecting and non-reflecting boundary conditions for simulation of compressible viscous flow," Journal of Computational Physics 213, 437-449, 2006.

[101] A. Widenhorn, B. Noll \& M. Aigner, "Accurate boundary conditions for the numerical simulation of thermoacoustic phenomena in gas-turbine combustion chambers," ASME Turbo Expo 2006.

[102] F. Mechel, Formulas of Acoustics, Springer, 2008.

[103] J. Miles, "Acoustic transmission matrix of a variable area duct or nozzle carrying a compressible subsonic flow," Journal of the Acoustical Society of America 58(6), 1577$1586,1981$.

[104] E. Dokumaci, "On transmission of sound in a non-uniform duct carrying a subsonic compressible flow," Journal of the Acoustical Society of America 210(3), 391-401, 1998. 
